\title{
Self-Assembly of Surface-Acylated Cellulose Nanowhiskers
}

\author{
Dissertation \\ to attain the doctoral degree (Dr. rer. nat.) \\ of the Faculty of Forest Science and Forest Ecology \\ Georg-August-Universität Göttingen \\ Submitted by \\ Huan Liu \\ born on the $14^{\text {th }}$ July 1990 in P.R. China
}

Göttingen, 2020 


\section{Thesis Committee}

Prof. Dr. Kai Zhang, Abteilung Holztechnologie und Holzwerkstoffe, Georg-AugustUniversität Göttingen

Prof. Dr. Philipp Vana, Makromolekulare Chemie, Georg-August-Universität Göttingen

Prof. Dr. Carsten Mai, Abteilung Holzbiologie und Holzprodukte, Georg-August-Universität Göttingen

\section{Members of the Examination Board}

\section{Reviewer:}

Prof. Dr. Kai Zhang, Abteilung Holztechnologie und Holzwerkstoffe, Georg-AugustUniversität Göttingen

\section{Second Reviewer:}

PD Dr. Markus Euring, AG Chemie und Verfahrenstechnik von Verbundwerkstoffen, GeorgAugust-Universität Göttingen

\section{Further members of the Examination Board:}

Prof. Dr. Carsten Mai, Abteilung Holzbiologie und Holzprodukte, Georg-August-Universität Göttingen 


\section{Acknowledgement}

First and foremost, I would like to express my sincere appreciation to my supervisor, Prof. Dr. Kai Zhang for his continuous support of my Ph.D. study and research, for his patience, motivation, and immense knowledge. He convincingly guided and encouraged me to be professional and do the right thing even when the road got tough. I appreciate all his contributions of time and ideas to make my Ph.D. experience productive and stimulating. The joy and enthusiasm he has for his research was contagious and motivational for me, even during tough times in the Ph.D. pursuit. I thank him for providing me with the opportunity to work with a talented team of young researchers.

Besides my supervisor, I would like to thank the members of my Ph.D. committee, Prof. Dr. Philipp Vana and Prof. Dr. Carsten Mai for their generous help in equipment supporting and helpful comments. My thesis committee guided me through all these years. I would like to thank my reading committee members for their time and valuable feedback on a preliminary version of this thesis. I also would like to thank the members of my oral defense committee for their time and insightful questions.

I would like to thank all my group members for all the great times that we have shared. I also greatly thank all my collaborators for their valuable discussion and generous helps in equipment supporting. Moreover, I would like to express my gratitude to all my friends in Goettigen. My time at Goettingen was enjoyable in large part due to the many friends that became a part of my life.

I would like to thank China Scholarship Council (CSC) for the financial support that made my Ph.D. work possible.

Last but not the least, I would like to thank my parents and my beloved boyfriend - Bo Pang for all their love and encouragement. 


\begin{abstract}
Self-assembly of nanoparticles into ordered materials is an attractive route for the synthesis of functional materials that are not feasible through conventional techniques. Recently, much attention has been paid to self-assembly of anisotropic cellulose nanowhiskers (CNWs) because of their unique properties, such as high surface to volume ratio, high mechanical performance, small thermal expansion coefficient, renewability, and sustainability. The objective of this research was to investigate the self-assembly of surfaceacylated CNWs and to fabricate functional materials via this path.
\end{abstract}

Helical fibers with the lengths of micrometers were fabricated by the evaporation-driven self-assembly of CNWs with surface-immobilized 10-undecenoyl groups (CNWs-SU). The self-assembly process and the final helical structures were affected by parameters including the wettability of substrates, dispersing solvents, the amount of 10-undecenoyl groups, the crystallinity, the dimension of CNWs, and the length of acyl chains. In particular, surfaceacylated CNWs with a certain amount of 10-undecenoyl groups (ca. $3.52 \mathrm{mmol} / \mathrm{g}$ ), an appropriate crystallinity (ca. 40\%), a length of about $135 \mathrm{~nm}$, and a diameter of around $4 \mathrm{~nm}$, preferentially self-assembled into explicit left-handed helical fibers from their THF suspensions on wafers.

The surface of CNWs-SU contains numerous olefin groups, allowing them to be further modified. After the photo-induced thiol-ene reaction on the terminal olefin groups with 1octadecanethiol, CNWs-SU-C18 were obtained. Janus films of CNWs-SU-C18 with or without graphene oxide (GO) were fabricated via one-step evaporation-driven self-assembly process, which have reconstructible time-dependent micro-/nanostructures and asymmetric wettability. The heterogeneous aggregation of CNWs on rough Teflon substrates favors the formation of uniform films, leading to hydrophobic smooth bottom surface. The homogeneous nucleation of residual CNWs in bulk suspensions promotes the growth of patchy microspheres with an average diameter of $22.7 \pm 2.1 \mu \mathrm{m}$, which precipitate on the top surface leading to enhanced hydrophobicity. These patchy microspheres are thermoresponsive and vanish after heating at $60{ }^{\circ} \mathrm{C}$ for few minutes, while they are reconstructed at room temperature with time-dependent evolving micro-/nanostructures in dry-state within two days. The thermo-responsive transition of patchy microparticles leads to accompanied switchable change between transparency and opacity of Janus films. Furthermore, the incorporation of GO generates more patchy microspheres with an average diameter of 
$13.5 \pm 1.3 \mu \mathrm{m}$ on the top surface of hybrid Janus films. Different distributions of CNWs and GO in Janus films and the solvent-responsive self-assembled patchy microparticles of CNWs facilitate their reversible actuation by showing very fast curling in THF within 6 seconds and flattening in water for at least 25 cycles.

The self-assembly of CNWs in confined geometries provides a powerful method to break the symmetry of an ordered structure, thus it can serve as an efficient route for the fabrication of novel morphologies. Three-dimensional (3D) ordered microporous CNWs-SU honeycomb films were prepared by breath figure (BF) method. The resulting films show high porous order over large regions and the iridescent color was only displayed by the rim of the obtained honeycomb film due to the confined 3D geometry drastically affects the self-assembly of CNWs, which is different with traditional dish-cast CNWs films showing the iridescent color over the whole area. In the confined 3D walls, CNWs self-assembly into cholesteric architectures which resulted in the iridescent color of the rims. Some critical influencing factors, such as the concentration of the CNWs solution and the relative humidity of the atmosphere were also investigated. Too low or too high concentrations of CNWs solutions/relative humidity are not preferable for the formation of uniform porous films and the self-assembly of CNWs in the confined geometry was also affected. This method provides a new idea to study the interactions of CNWs beyond what has been achieved in a planar geometry.

This thesis is a cumulative work including 3 publications. All papers were submitted in peer-reviewed journals, one of them was already published and two of them were submitted. The background, the objective of the study, results and discussion of the three publications and the conclusion are presented in Section 1-4. 


\section{Zusammenfassung}

Die Selbstorganisation von Nanopartikeln zu geordneten Materialien ist ein attraktiver Weg für die Synthese der funktionellen Materialien, die mit herkömmlichen Techniken nicht realisierbar sind. In jüngster Zeit wurde die Selbstorganisation von anisotropen CelluloseNanowhiskern (CNWs) aufgrund ihrer einzigartigen Eigenschaften wie hohen Verhältnis von Oberfläche zu Volumen, hoher mechanischer Leistung, geringer Wärmeausdehnungskoeffizienten, die Erneuerbarkeit und die Nachhaltigkeit eine große Aufmerksamkeit gewidmet. Das Ziel dieser Forschung war es, die Selbstorganisation von oberfläche acylierung CNWs $\mathrm{zu}$ untersuchen und über diesen Weg die funktionellen Materialien herzustellen.

Helix Fasern mit einer Länge von Mikrometern wurden durch verdampfungsbedingte Selbstorganisation von CNWs mit oberfläche immobilisierten 10-Undecenoylgruppen (CNWs-SU) hergestellt. Der Selbstorganisationsprozess und die endgültigen helikalen Strukturen wurden durch die Parameter wie die Benetzbarkeit von den Substraten, die dispergierenden Lösungsmittel, die Menge an 10-Undecenoylgruppen, die Kristallinität, die Dimension von CNWs und die Länge von Acylketten beeinflusst. Insbesondere selbstorganisierte sich die oberfläche acylierung CNWs mit einer bestimmten Menge an 10Undecenoylgruppen (ca. $3.52 \mathrm{mmol} / \mathrm{g}$ ), einer geeigneten Kristallinität (ca. 40\%), einer Länge von ca. $135 \mathrm{~nm}$ und einem Durchmesser von ca. $4 \mathrm{~nm}$; vorzugsweise selbstorganisiert zu expliziten linkshändigen helikalen Fasern aus ihren THF-Suspensionen auf Wafern.

Die Oberfläche von CNWs-SU enthält zahlreiche Olefin Gruppen, so dass sie weiter modifiziert werden können. Nach der photoinduzierten Thiol-En-Reaktion an den terminalen Olefingruppen mit 1-Octadecanthiol wurden CNWs-SU-C18 erhalten. Die Janus-Filme aus CNWs-SU-C18 mit oder ohne Graphenoxid (GO) wurden über einen einstufigen verdampfungsgesteuerten Selbstorganisationsprozess hergestellt, der rekonstruierbare zeitabhängige Mikro-/Nanostrukturen und asymmetrische Benetzbarkeit aufweist. Die heterogene Aggregation von CNWs auf rauen Teflon Substraten begünstigt die Bildung der gleichmäßigen Filme, was zu einer hydrophoben glatten Bodenoberfläche führt. Die homogene Keimbildung restlicher CNWs in Massen Suspensionen fördert das Wachstum von der fleckigen Mikrokugeln mit einem durchschnittlichen Durchmesser von 22.7 $\pm 2.1 \mu \mathrm{m}$, die auf der Oberseite ausfallen und zu einer erhöhten Hydrophobizität führen. Diese fleckigen Mikrokugeln sind thermo-responsive und verschwinden nach einigen Minuten Erhitzen auf 
$60{ }^{\circ} \mathrm{C}$, während sie bei Raumtemperatur mit zeitabhängig entwickelnden Mikro/Nanostrukturen im trockenen Zustand innerhalb von zwei Tagen rekonstruiert werden. Der thermo-responsive Übergang von fleckigen Mikropartikeln führt $\mathrm{zu}$ einem begleitenden Wechsel zwischen Transparenz und Opazität von Janus-Filmen. Darüber hinaus erzeugt der Einbau von GO die fleckigeren Mikrokugeln mit einem durchschnittlichen Durchmesser von $13.5 \pm 1.3 \mu \mathrm{m}$ auf der Oberseite von Hybrid-Janus-Filmen. Die unterschiedlichen Verteilungen von CNWs und GO in Janus-Filmen und die auf Lösungsmittel ansprechenden selbstorganisierten fleckigen Mikropartikel von CNWs erleichtern ihre reversible Aktivierung, indem sie innerhalb von 6 Sekunden eine sehr schnelle Kräuselung in THF zeigen und in Wasser für mindestens 25 Zyklen abflachen.

Die Selbstorganisation von CNWs in engen Geometrien bietet eine leistungsstarke Methode, um die Symmetrie einer geordneten Struktur zu brechen, und kann daher als effizienter Weg zur Herstellung neuartiger Morphologien dienen. Dreidimensionale (3D) geordnete mikroporöse CNWs-SU-Wabenfilme wurden nach der Methode der Atemfigur (BF) hergestellt. Die resultierenden Filme zeigen über große Bereiche eine hohe poröse Ordnung und die schillernde Farbe wurde nur durch den Rand des erhaltenen Wabenfilms angezeigt, da die begrenzte 3D-Geometrie die Selbstorganisation von CNWs drastisch beeinflusst, was sich von herkömmlichen CNW-Filmen aus Schalenguss unterscheidet, die letzteren die schillernde Farbe über den gesamten Bereich zeigen. In den begrenzten 3D-Wänden organisieren sich CNWs zu cholesterischen Architekturen, was zu einer schillernden Farbe der Felgen führte. Einige kritische Einflussfaktoren wie die Konzentration der CNW-Lösung und die relative Luftfeuchtigkeit wurden ebenfalls untersucht. $\mathrm{Zu}$ niedrige oder zu hohe Konzentrationen an CNW-Lösungen/relative Luftfeuchtigkeit sind für die Bildung der gleichmäßigen porösen Filme nicht vorzuziehen, und die Selbstorganisation von CNWs in der begrenzten Geometrie wurde ebenfalls beeinflusst. Diese Methode bietet eine neue Idee, um die Wechselwirkungen von CNWs über das hinaus zu untersuchen, was in einer planaren Geometrie erreicht wurde.

Diese Arbeit ist eine kumulative Arbeit mit 3 Publikationen. Alle Beiträge wurden in der von Experten begutachteten Fachzeitschriften eingereicht, eine davon wurde bereits veröffentlicht und zwei von ihnen wurden eingereicht. Der Hintergrund, das Ziel der Studie, die Ergebnisse und die Diskussion der drei Veröffentlichungen sowie die Schlussfolgerung sind in Abschnitt 1-4 dargestellt. 


\section{Table of Contents}

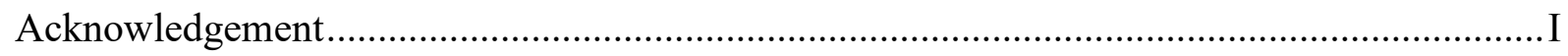

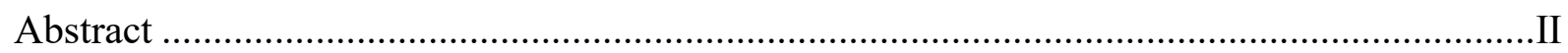

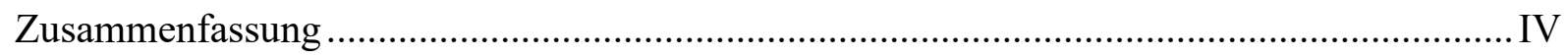

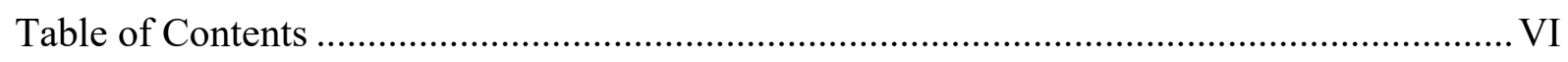

List of Publications........................................................................................................ VII

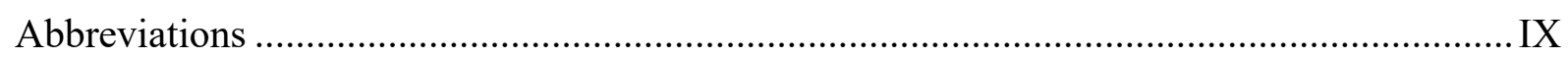

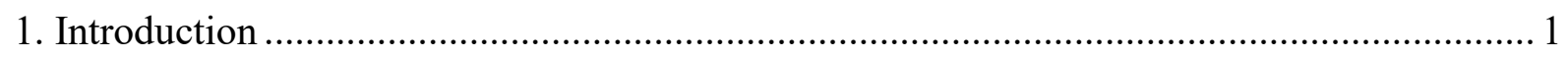

1.1. Chemical modifications of CNWs .................................................................. 2

1.1.1 Imparting ionic charges to CNWs ............................................................. 3

1.1.1.1 Carboxymethylation ............................................................................

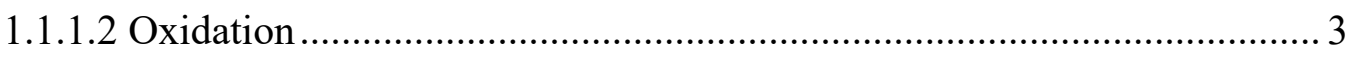

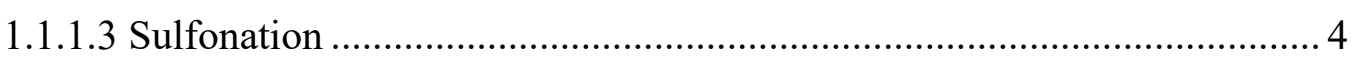

1.1.2 Generation of CNWs with non-polar moieties ................................................... 5

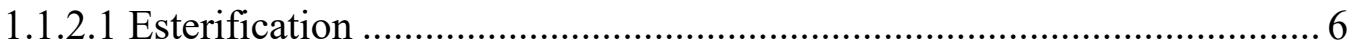

1.1.2.2 Etherification ..............................................................................

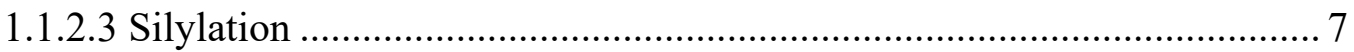

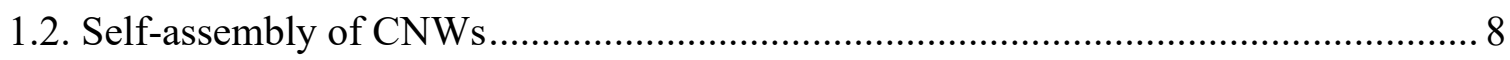

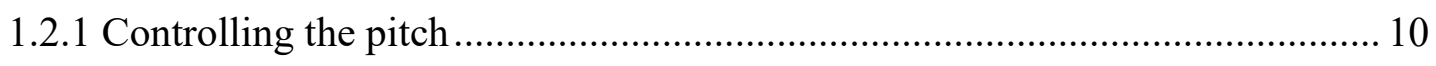

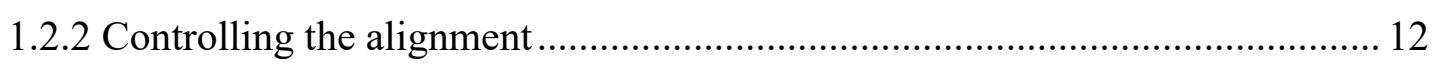

1.2.2.1 Shear induced alignment of CNWs .................................................. 12

1.2.2.2 Mechanical stress induced alignment of CNWs ...................................... 14

1.2.2.3 Electric field induced alignment of CNWs ........................................... 17

1.2.2.4 Magnetic field induced alignment of CNWs........................................... 17

1.2.3 Coassembly of CNWs and other nanoparticles .............................................. 18

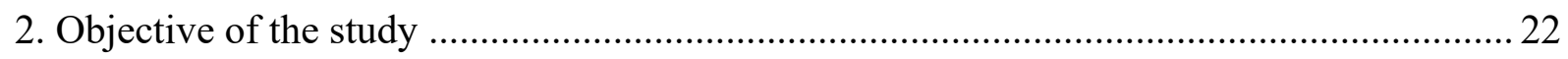

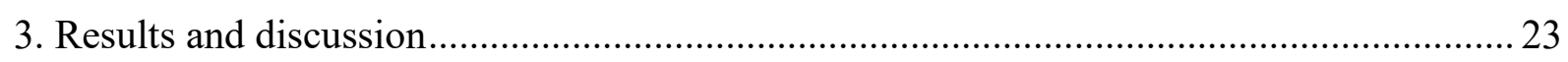

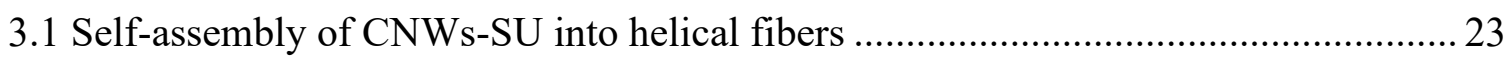

3.2 Self-assembly of surface-acylated CNWs for Janus films........................................... 30

3.3 Self-assembly of CNWs-SU confined in honeycomb films ....................................... 36

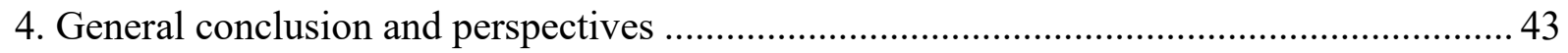

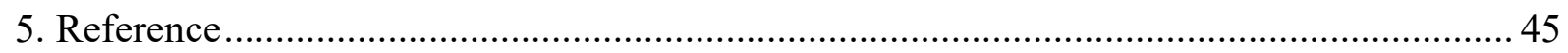

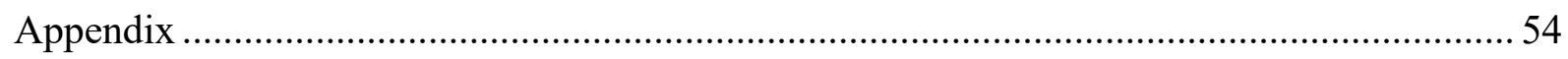




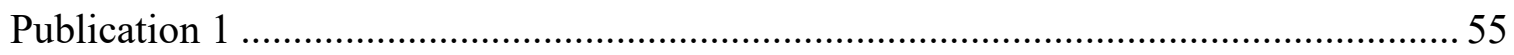

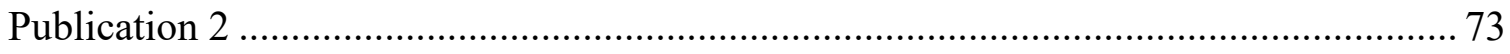

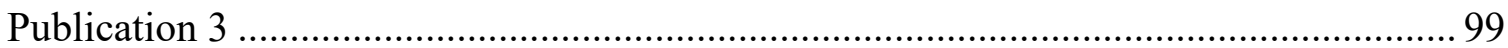




\section{List of Publications}

Publication 1

Helical fibers via evaporation-driven self-assembly of surface-acylated cellulose nanowhiskers

Huan Liu, Bo Pang, Renata Garces, Riza Dervişoğlu, Longquan Chen, Loren Andreas, and Kai Zhang*

Angewandte Chemie International Edition 2018, 57(50), 16323-16328.

Publication 2

Self-assembly of surface-acylated cellulose nanowhiskers and graphene oxide for multiresponsive Janus films with time-dependent dry-state structures

Huan Liu, Bo Pang, Qiyun Tang, Marcus Müller, Hua Zhang, Riza Dervişoğlu, and Kai Zhang*

Published online in Small (2020). DOI: 10.1002/smll.202004922

Publication 3

Self-assembly of surface-acylated cellulose nanowhiskers confined in honeycomb films

Huan Liu, Bo Pang, Kai Zhang*

Submitted 2020. 

Abbreviations
1D
One dimensional
2D
Two dimensional
$3 \mathrm{D}$
Three dimensional
AFM
Atomic force microscopy
AGUs
D-anhydroglucose units
$\mathrm{CNC}$
Cellulose nanocrystal
$\mathrm{CNF}$
Cellulose nanofiber
CNW
Cellulose nanowhisker
CNWs-SU
CNWs with surface-immobilized 10-undecenoyl groups
$\mathrm{CSE}_{3}$
Cellulose stearoyl ester with degree of substitution of 3
$\mathrm{CUE}_{3}$
Cellulose 10-undecenoyl ester with degree of substitution of 3
DCM
Dichloromethane
DS
Degree of substitution
FTIR
Fourier transform infrared spectroscopy
GO
Graphene oxide
LSM
Laser scanning microscope
$\mathrm{MCC}$
Microcrystalline cellulose
PCUE
Polymeric cellulose 10-undecenoyl ester
PDMS
Polydimethylsiloxane
PLM
Polarized light microscopy
SEM
Scanning electron microscopy
TEM
Transmission electron microscopy
THF
Tetrahydrofuran 


\section{Introduction}

Cellulose, a highly functionalizable polymer with many existing industrial applications, holds a crucial position in abundant organic raw materials due to its availability, biocompatibility, biological degradability, and sustainability. ${ }^{1-2}$ It is a linear natural polymer consisting of D-anhydro-glucose $\left(\mathrm{C}_{6} \mathrm{H}_{11} \mathrm{O}_{5}\right)$ repeating units joined by $1,4-\beta$ - $\mathrm{D}$-glycosidic linkages at $\mathrm{C} 1$ and $\mathrm{C} 4$ position (Figure 1). ${ }^{3}$ In nature, cellulose chains have a degree of polymerization (DP) of about 10000 glucopyranose units in wood cellulose and 15000 in native cotton cellulose. ${ }^{4}$ There are three hydroxyl groups on each monomer which result in the formation of a network of intra- and intermolecular bonds along the cellulose chain. ${ }^{5}$ Besides, a network of van der Waals connections is established between the chain layers. The van der Waals and intermolecular hydrogen bonds between hydroxyl groups and oxygens of adjacent molecules promote the arrangement and stabilization of the cellulose molecules into a highly organized structure through crystalline packing. ${ }^{6}$ It gives rise to the structures with a width of 2-20 nm and the length of up to a few microns crystalline rods along the microfibril axis. ${ }^{7}$ These crystalline domains are interspersed with amorphous regions in the fibril structure where the cellulose molecules could not be stabilized laterally through H-bondings. Compared with the crystalline parts, the density of the amorphous domains is much lower which results in the easily available for bonding (e.g. hydrogen) with other molecules including water. ${ }^{8}$ For some plant species, these amorphous regions can represent up to $50 \%$ of the structure, while in bacterial cellulose and cellulose extracted from some algae the crystalline domains correspond to almost $100 \%$ of the fibril. These crystalline domains are almost defect-free. ${ }^{7}$

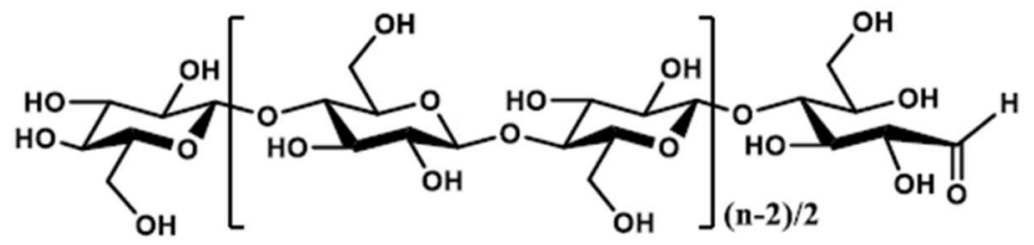

Figure 1. Molecular structure of cellulose.

Employing various reaction strategies, various types of nanomaterials can be extracted from cellulose owing to its hierarchical structure and semicrystalline feature. Generally, 
cellulose nanomaterials can be grouped into three broad categories: 1) cellulose nanowhiskers (CNWs), with other designation such as cellulose nanocrystals (CNCs), nanocrystalline cellulose (NCC), rod-like cellulose microcrystals; 2) cellulose nanofibers (CNF) or microfibrillated cellulose (MFC); and 3) bacterial cellulose (BC), also referred to as microbial cellulose. ${ }^{9-10}$

CNWs, a kind of fascinating bio-based nanoscale materials, have garnered a tremendous level of attention from many research communities owing to its unique structural features and impressive physicochemical properties such as biocompatibility, biodegradability, renewability, sustainability, low density, nontoxicity, stiffness, adaptable surface chemistry, optical transparency, and improved mechanical properties. ${ }^{8} \mathrm{CNWs}$ are broadly needle-shaped nanometric or rod-like particles and the size of CNWs can vary from $100 \mathrm{~nm}$ to several micrometers in length and 4 to $70 \mathrm{~nm}$ in width. The particles are $100 \%$ cellulose and highly crystalline, between $54 \%$ and $88 \% .{ }^{6}$ Normally, different sources of cellulosic materials and different reaction conditions result in different kinds of CNWs with different dimensions, morphologies, and degrees of crystallinity. ${ }^{1} \mathrm{CNWs}$ can be produced from diverse materials that include algal cellulose, bacterial cellulose, wheat straw, cotton linters, microcrystalline cellulose, tunicates, and wood pulp. ${ }^{11}$ Until now, many methods have been used to produce CNWs. Among various methods, one of the most frequently used technique to prepare CNWs from various cellulosic materials is acid hydrolysis, such as sulfuric and hydrochloric acids. ${ }^{12-}$

${ }^{15}$ Recently, other mineral and organic acids have also been utilized to produce CNWs, such as phosphoric, hydrobromic, nitric acids, and a mixture composed of hydrochloric and organic acids. ${ }^{16}$ Several other techniques also have been developed, such as mechanical refining, enzymatic hydrolysis, ionic liquid treatment, subcritical water hydrolysis, oxidation method and combined processes. ${ }^{8}$

\subsection{Chemical modifications of CNWs}

Due to the hydrophilic nature of cellulose, CNWs cannot be uniformly dispersed in most non-polar polymer media. To expand the application fields of CNWs, various covalent and non-covalent modification approaches have been used to develop new surface-modified CNWs with outstanding properties. ${ }^{8}$ According to its structure, the reactive sites on cellulose, where chemical reactions can be conducted, are the three-hydroxyl groups indicated as C2$\mathrm{OH}, \mathrm{C} 3-\mathrm{OH}$ and $\mathrm{C} 6-\mathrm{OH}$. C6-OH is a primary hydroxyl group, where most of the modification predominantly occurs. ${ }^{17}$ The main advantage of chemical modification is that the introduced 
either negative or positive electrostatic charges, and hydrophilic or hydrophobic chains on the CNW surface provide better dispersion in any solvent/polymer. It also helps to tune the surface energy characteristics of CNWs to improve compatibility, especially when used in conjunction with non-polar or hydrophobic polymer matrices. ${ }^{18}$ However, the main challenge for the surface modification of CNWs is to choose a method which can realize successful modification of CNWs surface, and effectively avoid any polymorphic conversion and maintain the integrity of the crystals. ${ }^{16}$

\subsubsection{Imparting ionic charges to $\mathrm{CNWS}$}

Three common routes for introducing ionic groups on the surface of CNWs, namely carboxymethylation, oxidation and sulfonation, are discussed here.

\subsubsection{Carboxymethylation}

After the surface modification by carboxymethylation, the surfaces of CNWs are negatively charged which promote the formation of stable suspension from carboxymethylated CNWs. Compared with the sulfate ester groups, the carboxyl groups are more stable under alkaline conditions which lead to a broader application of CNWs. ${ }^{19-20}$

Martin et al. performed carboxymethylated cellulose nanocrystals (CNC-COOH) obtained from $\mathrm{HCl}$ hydrolysis of carboxymethylated cellulose nanofibrils. ${ }^{21}$ The structural properties and aggregation behavior of carboxymethylated CNCs were analyzed with small angle neutron scattering (SANS), transmission electron microscopy (TEM), atomic force microscopy (AFM) and dynamic light scattering (DLS). The CNC-COOH system was shown to laterally aggregate into $2 \mathrm{D}$-stacks that was stable both in solution and when adsorbed to surfaces.

\subsubsection{Oxidation}

(2,2,6,6-Tetramethylpiperidine-1-oxyl)-mediated (TEMPO-mediated) oxidation of CNWs has been found to be an efficient route to convert the hydroxylmethyl groups to carboxylic form with high yields in the presence of a co-oxidizing agent. ${ }^{22}$ Generally, the oxidation is carried out with a catalytic amount of TEMPO with a secondary oxidant such as sodium hypochlorite or sodium chlorite to recycle the TEMPO. ${ }^{23}$

De Nooy et al. first reported that TEMPO-mediated oxidation just oxidize the hydroxymethyl groups of polysaccharides (i.e. starch, amylodextrin and pullulan), whereas 
the secondary hydroxyls remained unaffected. ${ }^{24-26}$ The TEMPO-mediated oxidation has been applied to different cellulose materials. Chang and Robyt obtained polymers with primary alcohol groups selectively oxidized by applying the TEMPO-NaBr-NaClO system to waterinsoluble polysaccharides, i.e. cellulose and chitin. ${ }^{27}$ The oxidized products prepared from cellulose samples had $84-87 \%$ oxidation yields of primary alcohol groups as were determined by the carbazole method.

Habibi et al. performed TEMPO-mediated oxidation of CNWs resulting from $\mathrm{HCl}$ hydrolysis of tunicate under various conditions. ${ }^{19}$ The effect of hypochlorite concentration on the outcome of the oxidation was investigated and it was shown that the hydroxymethyl groups on their surface were selectively converted to carboxylic group, but the CNWs still kept their initial morphological integrity and native crystallinity. Similar observations were reported by Fraschini et al. who also showed that carboxylate groups were selectively introduced onto the surface of CNWs up to a total acid content of $1200 \mathrm{mmol} / \mathrm{kg}$ without damaging the integrity of the crystals. ${ }^{22}$ They also found that the final acid content could easily be tuned by varying the amount of oxidant introduced. Montanari et al. prepared surface carboxylated CNWs with different sizes and degrees of oxidation by TEMPOmediated oxidation of cotton linters and microfibrils of parenchyma cell cellulose. ${ }^{28}$ They found that the crystal size was decreased due to some degradation in the amorphous areas of the starting material. The similar approach had been implemented by Li et al. who performed TEMPO-mediated oxidation of CNWs resulting from formic acid hydrolysis of bleached chemical pulp. ${ }^{29}$ They found that the modified CNWs with more ordered crystal structure and surface charge had better redispersibility and higher viscosity in aqueous which could be more effective when used as rheology modifier in the fields of water-based coating, paint, food, etc.

\subsubsection{Sulfonation}

Sulfonation is another approach to impart anionic charges to the surface of CNWs. Sulfonation of CNWs occurs during the process of the sulfuric acid-catalyzed hydrolysis through the esterification of the hydroxyl groups. ${ }^{11}$ However, it is very difficult to precisely control the amount of sulfate groups imparted to the surface of CNWs due to the esterification levels highly depend on the reaction conditions, such as hydrolysis time, temperature, and acid concentration. On the other hand, it is still challenging to quantify the resulting ester groups. ${ }^{30-31}$ To overcome these issues, some researchers have investigated post-treatment of CNWs obtained by hydrochloric acid hydrolysis with sulfuric acid to introduce controllable 
sulfate moieties on their surface. Araki et al. prepared a stable and highly viscous suspension of CNWs by postsulfating the HCl-hydrolyzed CNWs and controlled the surface charge from zero to various levels. ${ }^{32-33}$ In a similar way, a variety of CNWs with gradient degrees of sulfate groups were prepared by Lin and coauthors through the postsulfation and desulfation. ${ }^{34}$ They further explored the effect of the sulfation degree on surface chemistry, morphology, dimensions, and physical properties of different CNW samples. Wang et al. reported a novel kind of spherical CNWs suspension which was prepared by hydrolysis of microcrystalline cellulose with a mixture of sulfuric acid and hydrochloric acid under ultrasonic treatment. ${ }^{14}$ The formation of spherical CNWs was attributed to the combination of the mixed acid and the ultrasonic treatment. The suspensions with various concentrations showed different colors and liquid crystalline structures.

It has been found that sulfate groups introduced during hydrolysis with sulfuric acid, are suspected to diminish the thermostability. Roman et al. studied the relationship between the hydrolysis conditions, the number of sulfate groups introduced, and the thermal degradation behavior of CNWs. ${ }^{35}$ They found that a small amount of sulfate group results in the decrease of degradation temperatures. Although the presence of these sulfate groups on the surface of CNWs compromises their thermal stability, they are easy to disperse in aqueous solvents forming stable colloidal suspensions due to electrostatic repulsion.

Another method, such as phosphorylation, incorporating phosphate ester groups onto a cellulosic backbone, was also a well-known surface modification strategy for producing suitable materials for applications in diverse areas. ${ }^{36-37}$

\subsubsection{Generation of CNWs with non-polar moieties}

The hydrophilic surfaces cause CNWs to aggregate in organic solvents and the compatibility problems can also arise when CNWs are used in combination with commonly used hydrophobic polymers. ${ }^{38}$ In addition, once the CNW is dried, it is difficult to re-disperse CNW powders due to the agglomeration of hydrogen bonding between CNWs. ${ }^{39}$ To overcome these shortcomings, various chemical modification methods have been used to transform the CNW surface from hydrophilic to hydrophobic, while maintaining the integrity of its crystalline core. 


\subsubsection{Esterification}

Esterification has been widely used to modify the hydroxyl groups on the surface of CNWs due to its ease and directness. Among various esterification reactions, acetylation of CNWs is the most widely investigated.

It has been reported that polysaccharides such as starch, cyclodextrins, or cellulose can be successfully esterified under homogeneous or heterogeneous conditions by reaction with vinyl esters. ${ }^{40-44}$ Çetin et al. reported a novel and straightforward method for the production of surface acetylation of CNWs obtained from cotton linters by transesterification of vinyl acetate using potassium carbonate as the catalyst. ${ }^{45}$ The reaction was carried out in DMF at $95{ }^{\circ} \mathrm{C}$, and under moderate conditions, only the surface of the nanowhiskers was modified, while their dimensions and crystallinity remained unchanged. With prolonged reaction time, diffusion mechanisms controlled the rate, leading to nanowhiskers with higher levels of acetylation, smaller dimensions, and lower crystallinity. The resulted surface sufficiently acetylated CNWs form stable suspension in THF.

A simple and environmentally friendly chemical modification approach to confer high hydrophobicity to crystalline cellulose surface was reported by Yuan et al. ${ }^{46}$ With low reagent consumption and simple treatment procedures, highly hydrophobic whiskers were obtained. An alkenyl succinic anhydride (ASA) aqueous emulsion was mixed with tunicin whiskers, followed by complete drying and heating at $105{ }^{\circ} \mathrm{C}$. The degree of esterification was evaluated by FTIR spectrometry, elemental analysis, weight gain, and X-ray photoelectron spectroscopy (XPS). The surface-acylated whiskers retained their morphological and crystalline integrity. They were readily dispersible in solvents of low polarity and could also be well dispersed in polystyrene to form a nanocomposite due to their surface acylation.

The simultaneous occurrence of cellulose hydrolysis and acetylation of hydroxyl groups has also been reported. Braun et al. reported a single step to hydrolysis the amorphous region of cellulose chains and esterify the accessible hydroxyl groups simultaneously to produce surface functionalized CNWs. ${ }^{47}$ The reaction was carried out in an acid mixture composed of hydrochloric and organic acid (acetic and butyric acid). Resulting CNWs were of similar dimensions and more than half of the hydroxyl groups located on the CNWs surfaces were substituted under the employed reaction conditions. The resulting surface modified CNWs were dispersible in ethyl acetate and toluene indicating the increased hydrophobicity. 
Esterification of CNWs with organic fatty acid chlorides, having aliphatic chains of different lengths $\left(\mathrm{C}_{12}\right.$ up to $\left.\mathrm{C}_{18}\right)$, has been reported. Junior de Menezes et al. reported that the CNWs extracted from ramie fibers by acid hydrolysis were chemically modified using organic acid chloride aliphatic chains of different sizes (hexanoyl chloride, lauroyl chloride and stearoyl chloride). ${ }^{48}$ FTIR and XPS were used to examine the successful modification of CNWs. After the modification, the initial crystalline structure was preserved. Liu et al. reported a one-step technique to produced hydrophobic CNWs. CNWs with surfaceimmobilized 10-undecenoyl groups (CNWs-SU) were synthesized from microcrystalline cellulose after the surface esterification by 10-undecenoyl chloride under heterogeneous conditions and separated via ultracentrifugation. ${ }^{49}$ The degree of substitution of surface modified CNWs was about 1.32 based on the elemental analysis. The crystallinity of CNWs was about $40 \%$.

\subsubsection{Etherification}

Etherification is another important chemical modification to introduce cationic charges on the surface of CNWs. The surface of CNWs resulting from sulfuric acid hydrolysis of cotton was rendered cationic with epoxypropyltrimethylammonium chloride has been reported by Hasani et al. ${ }^{50}$ After the modification, CNWs retained their crystalline morphology and dimensions while resulting in a slight decrease in total surface charge density. Zaman et al. reported a semi-dry method to modify the surface of CNWs using glycidyltrimethylammonium chloride (GTMAC) as a cationization agent. ${ }^{51}$ An optimum reaction condition was obtained by changing reaction parameters, such as water content, reaction media, and reactant mole ratio on the performance of the quaternization process.

\subsubsection{Silylation}

Silylation has proven to be an efficient way to modify CNWs surface. Many researchers have attempted to graft silane onto CNWs. Goussé et al. partially silylated CNWs extracted from the acid hydrolysis of tunicate by a series of alkyldimethylchlorosilanes, with the carbon backbone of the alkyl moieties ranging from a short carbon length of isopropyl to longer lengths represented by n-butyl, n-octyl, and n-dodecyl. ${ }^{52}$ They found that when the degree of substitution (DS) of the order was $0.6 / 1$, the whiskers kept their morphological integrity, but due to their surface silylation, they became readily dispersible in solvents of low polarity such as THF. However, with a DS bigger than 1, the core of the whiskers became silylated, leading to the loss of their whisker character. At this level, it was no longer possible to obtain any 
birefringent suspension. A similar study performed by Pei et al. shown CNWs resulted from the acid hydrolysis of cotton were functionalized by partial silylation through reactions with n-dodecyldimethylchlorosilane in toluene. ${ }^{53}$ Such silylated CNWs formed stable suspensions in THF or chloroform and form stable homogeneous suspensions. However, the classical grafting processes with silane are generally carried out in a mixture of organic solvent and water which is the major limitation for use of the silane at large scale and entails health and environmental concerns. ${ }^{54}$ Khanjanzadeh et al. reported a direct, simple and straightforward method to functionalize CNWs with 3-aminopropyltriethoxysilane (APTES), without using hazardous solvents. ${ }^{55}$ APTES was firstly hydrolyzed in water and then adsorbed onto CNWs through hydrogen bonds, finally the chain hydrocarbon was covalently linked to the surface of CNWs through Si-O-C bonds which formed via the condensation reaction between hydroxyl and silanol groups. Silylated CNWs exhibited good thermal stability and a greater amount of residual char was formed at $500{ }^{\circ} \mathrm{C}$ compared to non-chemically modified CNWs.

\subsection{Self-assembly of CNWs}

In 1959, Marchessault et al. first reported the birefringent character of CNWs suspensions derived from sulfuric acid hydrolysis. ${ }^{56}$ However, the chiral nematic (cholesteric) phase of CNWs suspension was discovered until 1992 by Revol et al. ${ }^{57}$ The chiral nematic phase is characterized by the long-range orientational order of the nanorods combined with a helical modulation of the direction in which they align. ${ }^{58} \mathrm{CNWs}$, the helix is found to be always lefthanded and is attributed to the chirality of the interaction between the nanorods. However, the underlying mechanism is still under debate. ${ }^{59-62}$ The distance along the helical axis that separates nanorods of the same orientation after a $360^{\circ}$ rotation is commonly defined as the cholesteric pitch, $\mathrm{p} .{ }^{10}$

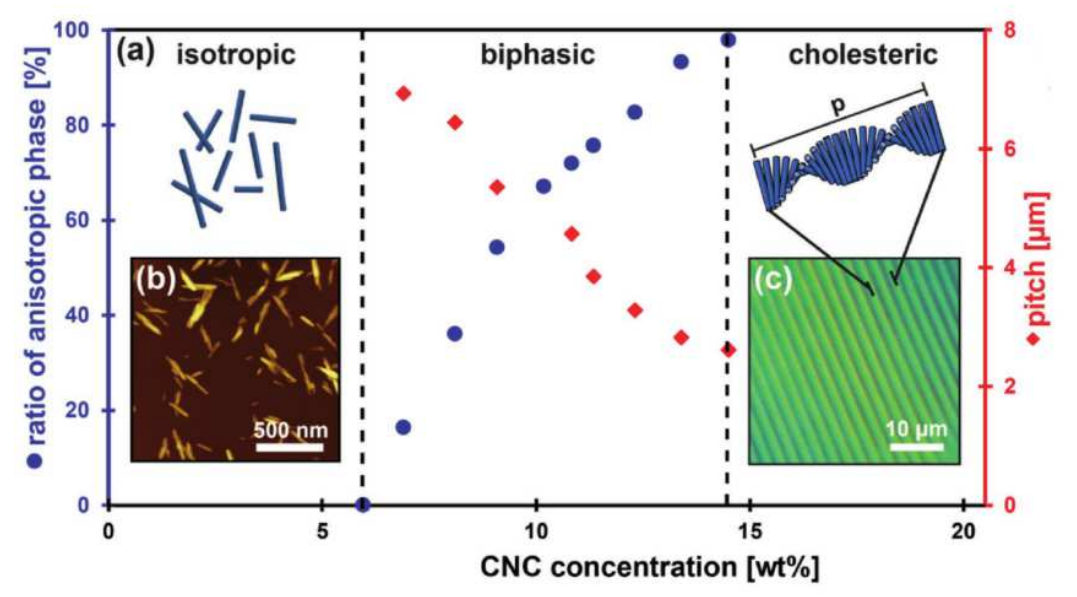


Figure 2. Phase diagram showing the transition from isotropic to cholesteric phase (blue dots) upon increasing CNWs concentration and the corresponding equilibrium pitch (red diamonds). (Reproduced with modification from PARKER et al. ${ }^{63}$ Copyright from WILEY-VCH 2017)

The phase transition diagram of pure CNWs suspension is given in Figure 2. In a dilute regime (isotropic phase), the CNWs are randomly oriented and the suspension displays a single isotropic phase. As the suspension concentration rise above a critical point, a second phase, to give an upper isotropic phase and a lower anisotropic phase. The volume of the lower anisotropic phase increased with the total suspension concentration increase. When the suspension reaches a critical concentration, the isotropic phase disappeared completely and the whole suspension was anisotropic. ${ }^{63-64}$ The texture of the liquid crystal observed through a polarized light microscope (PLM) depends on the orientation of the optical axis of the helicoidal assembly relative to that of the microscope. ${ }^{65}$ Figure 3 shows a typical image of an aqueous CNWs suspension with regions (a) of planar texture and (b) of fingerprint texture. The planar structure indicates that the screw axis of the CNWs liquid crystal is perpendicular to the substrate, leading to a birefringence phenomenon, while the fingerprint structure indicates that the axis is parallel to the substrate to form alternatively bright and dark stripes. $^{66-68}$

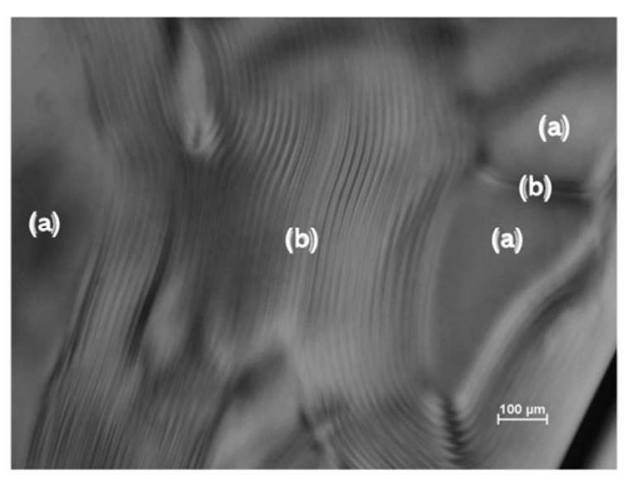

(a)

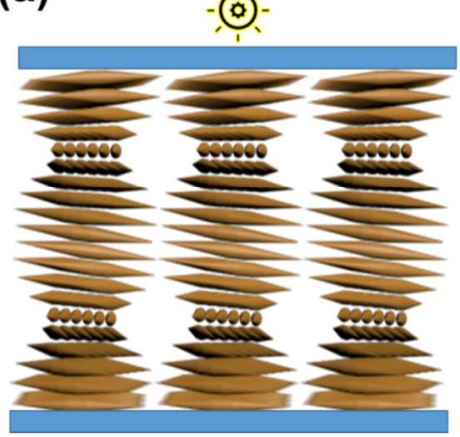

(b)

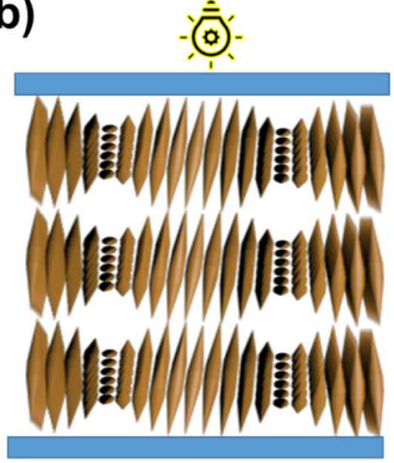

Figure 3. Regions (a) show planar texture and (b) show fingerprint texture. (Reproduced with modification from GRAY et al. ${ }^{65}$ Copyright from MDPI 2015) 


\subsubsection{Controlling the pitch}

The evaporation of aqueous suspension of CNWs produces iridescent chiral nematic film with a reflection color at visible wavelengths. A key issue is to control the chiral nematic pitch, thereby controlling the reflection color of CNWs film. ${ }^{69}$ Various factors may influence the self-assembly of CNWs from aqueous suspension to solid film.

Beck et al. found that the chiral nematic pitch of the suspensions decreased with increasing cellulose concentration. ${ }^{64}$ This is consistent with other findings that increasing the concentration of the suspension increases the coexisting concentrations in both isotropic and anisotropic phases, but the chiral nematic pitch of the anisotropic phase decreases. Furthermore, they measured the chiral nematic pitch at a total cellulose concentration of $7 \mathrm{wt} \%$ for all suspensions, which have varying average nanocrystal lengths. It was found that the pitch increased with the increase of rod length. Therefore, the longer rods showed less tendency to form the twisted chiral nematic structure, probably because at the same volume fraction of rods in the suspension, longer rods show a greater tendency to align in a parallel (untwisted) arrangement. ${ }^{70}$ Two main mechanisms have been used to explain the decrease in $p$ as CNWs concentration increases. First, the more CNWs present in the suspension, the stronger the total force for the twisting will be. Second, due to the CNW rods are charged, the increasing ionic strength upon increasing the particle concentration will decrease the range of the exponentially decaying electrostatic repulsion, thereby allow the rods to approach each other more closely. ${ }^{59}$

The temperature can also influence the chiral nematic structure in the suspension of CNWs. ${ }^{71}$ Evaporating an initially isotropic suspension at higher temperatures leads to significantly higher drying rates and thus decreases the pitch in the film. ${ }^{71}$ Beck et al. produced predefined patterns in solid iridescent films of CNWs by differential heating of aqueous CNWs suspensions during film casting. ${ }^{72}$ They found that the patterned areas are of different thickness and different chiral nematic pitch than the surrounding film due to the heating results in thicker areas of longer pitch. Differential heating of aqueous CNWs suspensions results in different evaporation rates and thermal motion in the areas of the CNWs suspension corresponding to the pattern-producing object and the surrounding, unperturbed suspension.

Sonication treatment is usually used in the preparation of CNWs suspensions to favor their dispersion. However, it is observed that the chiral nematic pitch increases with energy input. 
The effects of sonication on CNWs suspension properties have been studied by Dong and coworkers, who found that short-term sonication was sufficient to disperse CNWs, while further sonication was counterproductive because it increases the critical concentration required to form liquid crystalline phase. ${ }^{73}$ In order to develop a practical method for changing and controlling the iridescent color of CNWs films, Baker et al. evaluated the effect of ultrasonic energy treatment of aqueous CNWs suspensions before casting into films. ${ }^{74}$ Providing ultrasonic energy input to the CNWs suspension before film casting increases the pitch of the chiral nematic phase in suspension and moves the reflection band of the final iridescent film to longer wavelengths.

The ionic strength in a CNWs suspension can affect the formation of the anisotropic phase due to the negative charge on the surface of CNWs. By changing the ionic strength of the solution, the pitch can be controlled, thereby controlling the reflected color of the resulting films. Pan et al. studied the effect of ionic strength on the chiral nematic phase of CNWs extracted from woody biomass using acid hydrolysis. ${ }^{71}$ An appropriate amount of $\mathrm{NaCl}$ stock solution was mixed with $\mathrm{CNW}$ suspension before evaporation. They found that the pitch decreases with the increase of $\mathrm{NaCl}$ concentration. This is primarily due to the strong interaction between the sodium ions in $\mathrm{NaCl}$ and the surface of $\mathrm{CNWs}$, which shields the surface charges and reduces the effect of Coulombic repulsion. This phenomenon was also revealed by Dong and coauthors. ${ }^{64}$ They also found that in a biphasic sample with a fixed total concentration of crystallites, increasing the amount of added electrolyte reduced the formation of anisotropic phase, but the coexisting concentrations of the two phases only slightly increased. Dong and co-authors also studied the effect of counterions on ordered phase formation in suspensions of charged CNWs. ${ }^{75}$ They found that, for inorganic counterions, the critical concentration for ordered phase formation increases in the order of $\mathrm{H}^{+}<\mathrm{Na}^{+}<\mathrm{K}^{+}<$ $\mathrm{Cs}^{+}$. The properties of the counterions also affect other properties of the suspensions, such as their stability, the temperature dependence of the phase separation and of the chiral nematic pitch, and the redispersability of dried samples made from the suspensions.

The addition of a non-volatile solute also influences the equilibrium pitch of the cholesteric suspension. Mu et al. added D-(+)-glucose to the CNWs suspension and found that the addition of $\mathrm{D}-(+)$-glucose causes the pitch of chiral nematic long-pitch suspensions to decrease but shifts the (much shorter) pitch of the dry films to longer values, thus moving the iridescent reflection of the films to the red end of the spectrum. ${ }^{69}$ There were two distinct stages in changing the pitch during the evaporation process. In the first stage, the pitch 
decreases with the increase of concentration due to evaporation. At this stage, the addition of glucose causes a decrease in pitch. In the second stage, with the evaporation of solvent, the concentration of CNWs reached where the formation of ordered gels and glasses prevents further major changes in pitch. At this stage, the addition of glucose lowers the CNWs concentration and leads to an increase in pitch.

\subsubsection{Controlling the alignment}

Various techniques have been used to align CNWs in aqueous media and fixed the orientation state in the dried films or polymer composites, for the purpose of making their properties anisotropic and upgraded. These techniques include shear-based alignment, mechanical stress, and the allocation of intense magnetic or electric fields and so on.

\subsubsection{Shear induced alignment of CNWs}

Application of shear stress is an efficient method for manipulation of macro-scale orientation of the CNWs during the drying process. This method normally yields homogeneous films with CNWs aligned parallel to the shear flow direction. Consequently, it also inhibits the formation of the helical modulation. ${ }^{76}$

The alignment of CNWs in colloidal aqueous suspension under shear was characterized by using small angle synchrotron radiation scattering. ${ }^{77}$ In the isotropic phase, the average orientation of CNWs under low shear $\left(0.5 \mathrm{~s}^{-1}\right)$ was normal to the shear plane, while at high shear $\left(500 \mathrm{~s}^{-1}\right)$ it was parallel to the shear direction. This study indicates that the orientation of CNWs could be controlled by adjusting the shear rates. The order parameter for both isotropic and anisotropic configurations were investigated by Chowdhury et al using UV-vis spectrophotometry. Self-standing CNW films with various order parameters were prepared using shear flow with varying shear rates. The order parameters of different crystalline arrangements were calculated by determining the linear dichroitic ratio, evaluated from the transmitted light intensity of the CNW films when placed between cross polarizers. ${ }^{78}$ 

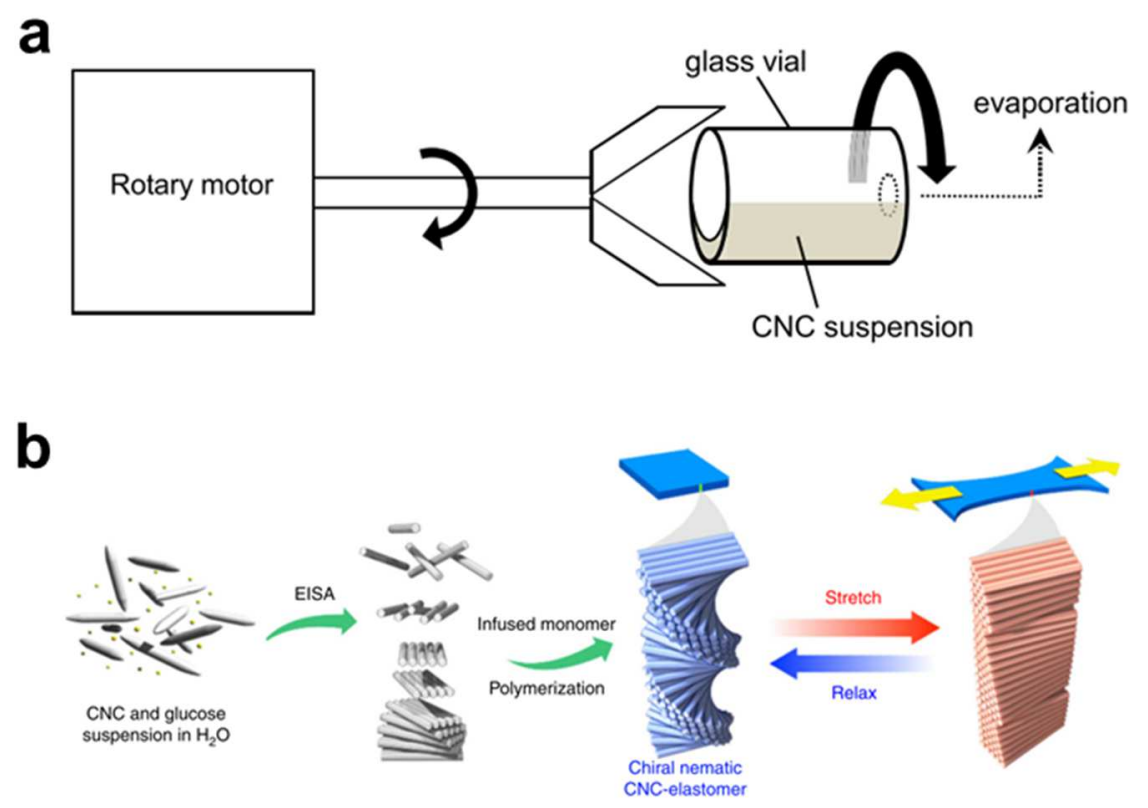

Figure 4. a) Schematic diagram of the experimental setup for shear-induced CNW alignment in a glass vial. (Reproduced from TATSUMI et al. ${ }^{80}$ Copyright from Springer Nature 2015) b) Reorientation of CNW particles from chiral nematic state to aligned structure by stretching. (Reproduced from KOSE et al. ${ }^{84}$ Copyright from Springer Nature 2019)

The alignment of CNWs under rotational shear was well investigated. An early work by Yoshiharu et al. reported on the preparation of CNWs film with high uniaxial orientation using a rotating glass vial. ${ }^{79}$ The highly ordered film was prepared by using the CNWs dispersion below the critical concentration for isotropic ordering and by drying the film under rotation. Similar work has been reported by Tatsumi et al. ${ }^{80} \mathrm{CNWs}$ films with different orientations were prepared by shearing suspensions in a glass vial under air drying (Figure 4a). While the orientation pattern of CNWs in the films was much affected by $\mathrm{pH}$ condition of the starting suspension. The alignment direction was perpendicular to the shear direction in the acid condition $(\mathrm{pH}=2.0)$, and the alignment direction was parallel to the shear direction in the neutral condition of $\mathrm{pH}=6.7$. Zong and coauthors also demonstrated that CNWs perpendicularly aligned to the shear orientation in a PET coated vial running at $1000 \mathrm{rpm}$ for 0.5 h. $^{81}$ 


\subsubsection{Mechanical stress induced alignment of CNWs}

The application of mechanical stress is critical for both the pitch and the alignment of the final nanostructure. Significant CNWs alignment was detected for both the wet and dry allcellulose nanocomposites at high strain. ${ }^{82}$ Applying tensile load favors alignment of the nanorods in the direction of the induced stretch. The reorientation of CNWs in agarose hydrogels stretched by micro tensile machine under different humidity conditions were systemically investigated by Osorio-Madrazo et al. ${ }^{83}$ They found that a controlled drying of the hydrogel is essential to establish interactions between the matrix and the CNWs which allow for a stress transfer during stretching and thereby promote their alignment.

However, pure CNWs films are brittle and thus changing the alignment of CNWs through mechanical stretching is not feasible. Therefore, incorporate CNWs into elastomers is a convenient method to obtain highly stretchable composite with aligned CNWs. Kose et al. studied the alignment of CNWs with a chiral nematic structure in the CNW/elastomer composite was tuned by stretching and relaxing (Figure $4 \mathrm{~b}$ ). Furthermore, the robust chiral nematic structure unwinds into a pseudo-nematic arrangement when the composite is stretched. The composite presents brilliant interference colors between crossed polarizers in response to extension and contraction. This kind of composite can be used to fabricate reversible stimuli-responsive materials for applications in flexible optics and sensors. ${ }^{84}$ They also found that when the pseudonematic CNWs elastomer was stretched parallel to the CNWs alignment direction, the CNWs became further aligned and the birefringence of the materials increases. In contrast, when the composite was stretched perpendicular to the CNWs alignment direction, the CNWs became more disordered and the birefringence decreases. ${ }^{85}$ 
a

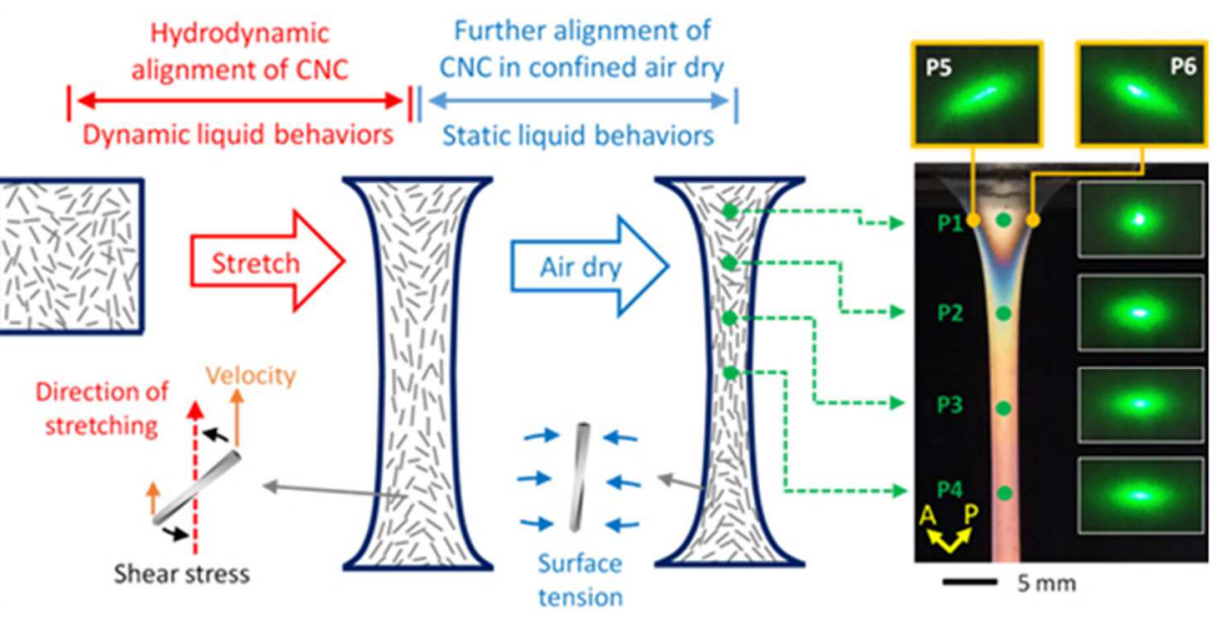

b

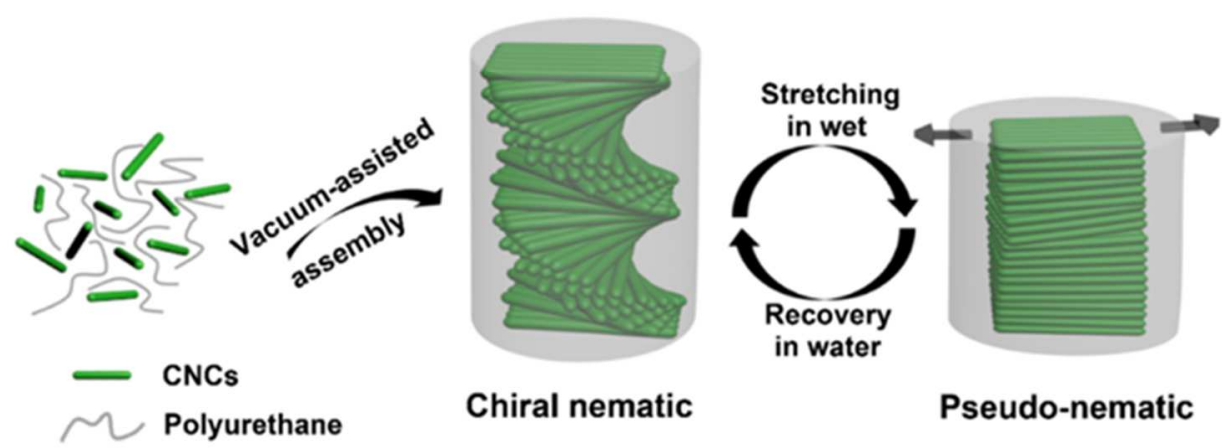

Figure 5. a) Schematic illustration for the hydrodynamic alignment of CNWs in the dynamic hydrogels under stretching. (Reproduced with modification from HUANG et al. ${ }^{86}$ Copyright from American Chemical Society 2019) b) Processing of shape and optical memory chiral nematic composite CNW/PU films and the reversible structural transitions between the chiral nematic and layered pseudonematic order upon stretching and recovery. (Reproduced from QU et al. ${ }^{87}$ Copyright from American Chemical Society 2019)

Dynamic hybrid hydrogels with multidimensional birefringence were fabricated using CNWs with hydrodynamic alignment by a shear-thinning phenomenon in the hydrogel network (Figure 5a). ${ }^{86}$ The CNWs preserve the hydrodynamic alignment when the stretching was terminated due to the reconstruction of hydrogel networks was much faster than the dissipation of CNWs orientation. The orientation index of CNW was further enhanced by the surface tension of hydrogels during the drying process, which results in anisotropic behavior with tunable birefringence. Qu et al. investigated the reversibly controllable optical property of chiral photonic CNW/PU films by switching the nanostructure between the chiral nematic and layered pseudonematic order. The stability of the composite film and the reorganization 
of $\mathrm{H}$ bonds in water gave rise to responsive mechanical adaption and increased ductility. The initial composite film transformed from the chiral nematic to layered pseudonematic structure when deformed to a stretched and fixed shape (Figure $5 \mathrm{~b}$ ). The fixed layered pseudonematic structure recovered in water to the original chiral nematic order, together with shape recovery to its initial state. These intriguing shapes and optical switchable materials will further advance the study and design of smart optical and mechanical sensors. ${ }^{87}$
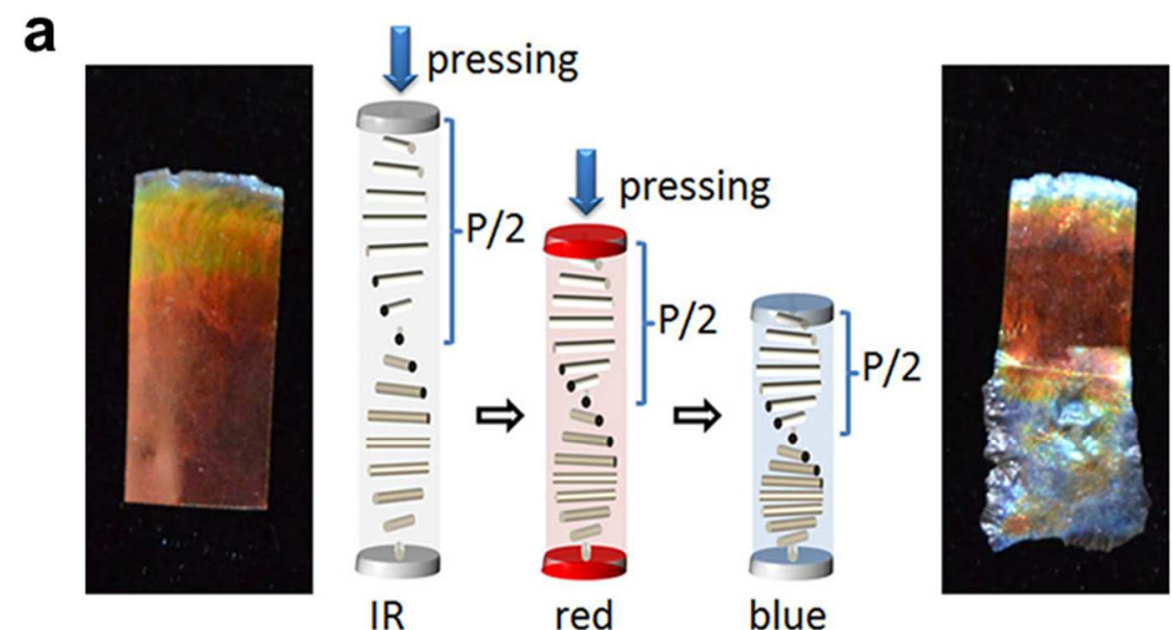

b
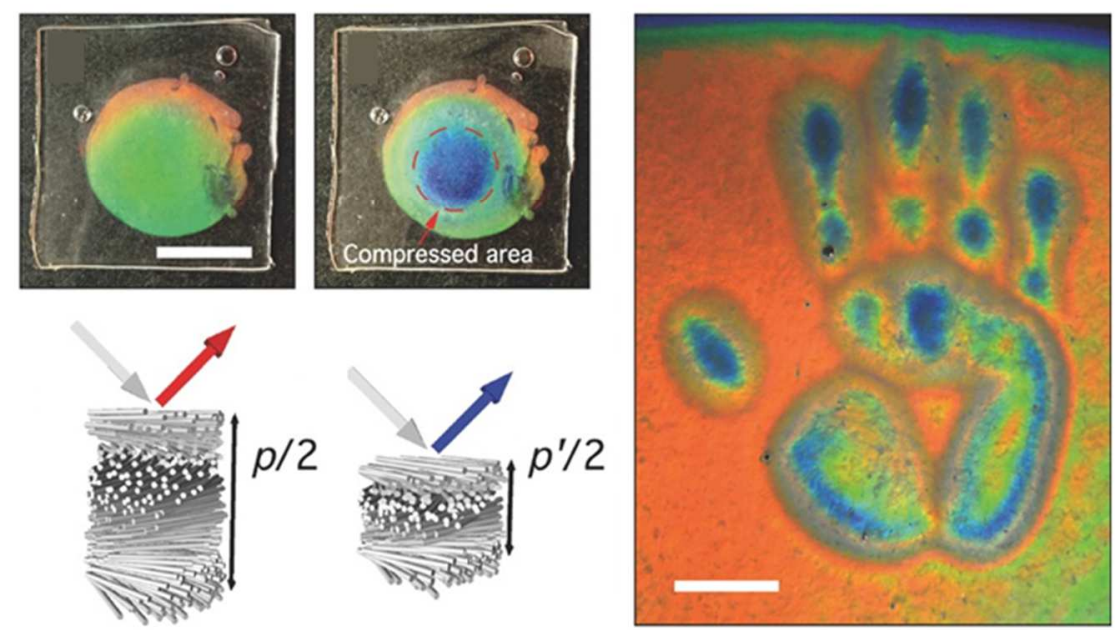

Figure 6. a) Schematic illustration of the change in helical pitch that occurs upon pressing the material. (Reproduced with modification from GIESE et al. ${ }^{88}$ Copyright from American Chemical Society 2013) b) Schematic illustration of the chiral HPC touch sensor based on the change in the pitch length. (Reproduced with modification from KAMITA et al. ${ }^{89}$ Copyright from WILEY-VCH 2016) 
Similarly, the alignment of CNWs can be controlled by applying mechanical compression. The color of the composite films can be manipulated by applying pressure to the materials, which causeed a blue shift in the wavelength of light reflected from the materials (Figure $6 \mathrm{a}$ ). Furthermore, the chiral nematic patterns can be imprinted into the composite films by using a stamp. These materials can essentially form a new type of imprintable photonic resin that may be useful for security features and decorations. ${ }^{88}$ Kamita et al. developed a method of producing a photonic strain sensor from aqueous LC-HPC phases. The color shift of the sensors from green to blue is a result of a change in the pitch of the cholesteric structure of HPC triggered by the imposed compressive deformation (Figure $6 \mathrm{~b}$ ). ${ }^{89}$

\subsubsection{Electric field induced alignment of CNWs}

Extern electric fields are an efficient and convenient tool to control the alignment of CNWs, both in aqueous or apolar suspension. Bordel et al. reported that CNWs were aligned along the direction of the electric field, and the birefringence of the suspension quickly vanished when the electric field was switched off. ${ }^{90}$ Moreover, the frequency and the strength of the applied electric field greatly influence the alignment of CNWs generated films as reported by Habibi et al. Uniform CNWs alignment in films obtained by drying CNW suspensions in the presence of electric fields with frequencies in the $\mathrm{kHz}-\mathrm{MHz}$ range and an amplitude of $\sim 2 \mathrm{kV} \mathrm{cm}^{-1} .{ }^{91}$ Ultrathin films of highly oriented CNWs were fabricated by using a convective/shear forces coupled with low intensity electric fields. ${ }^{92}$ The orientation and degree of alignment of the rod-like particles can be controlled by changing the applied field strength and frequency during film formation. This method not only allows precise tuning of the pitch but also enables sample homogeneity at the macroscopic scale as well as dynamic control of the structural colors. ${ }^{93}$

\subsubsection{Magnetic field induced alignment of CNWs}

Unlike electric fields, magnetic fields locally induce a perpendicular alignment of CNWs nanorods, due to their negative diamagnetic susceptibility. Sugiyama et al. first demonstrated that dilute aqueous suspensions of crystalline cellulose extracted from tunicate could be strongly oriented when subjected to a magnetic field of $7 \mathrm{~T} .{ }^{94}$ The crystals axis orientation perpendicular to the magnetic field direction, which was confirmed by electron and X-ray diffraction. This orientation of CNWs derives from the intrinsic anisotropic magnetic susceptibility of the individual $\mathrm{C}-\mathrm{C}, \mathrm{C}-\mathrm{O}, \mathrm{C}-\mathrm{H}$, and $\mathrm{O}-\mathrm{H}$ bonds from $\mathrm{CNW}$ and their relative orientation in the crystal. Recently, the use of moderate magnetic fields (e.g., 0.5-1.2 T) has 
been shown to successfully induce alignment in the chiral nematic phase. The effects of comparatively weak magnetic fields $(0-1.2 \mathrm{~T})$ and CNWs concentration $(1.65-8.25 \mathrm{wt} \%)$ on the kinetics and degree of CNWs ordering has been investigated using small angle X-ray scattering. ${ }^{95}$ In the $1.2 \mathrm{~T}$ magnetic field of the CNWs suspension above critical concentration, partial alignment occurred in less than 2 minutes, and then within 200 minutes, the slower collaboration sequence achieved near perfect alignment. Near-perfect alignment was also achieved at a lower magnetic field $(0.56 \mathrm{~T})$, yet the ordering was $36 \%$ slower. However, no magnetic alignment was detected for the suspension below critical concentration. Similarly, Frka-Petesic et al. use small commercial magnets $(\approx 0.5-1.2 \mathrm{~T})$ to control the orientation of the chiral nematic domains in suspension and to produce colored CNWs films with unique control over their final optical properties. ${ }^{96}$

\subsubsection{Coassembly of CNWs and other nanoparticles}

a

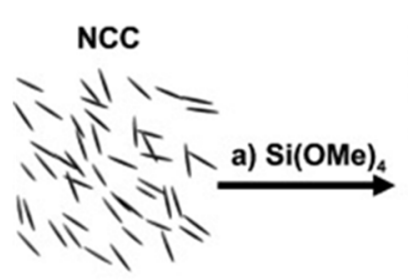

b

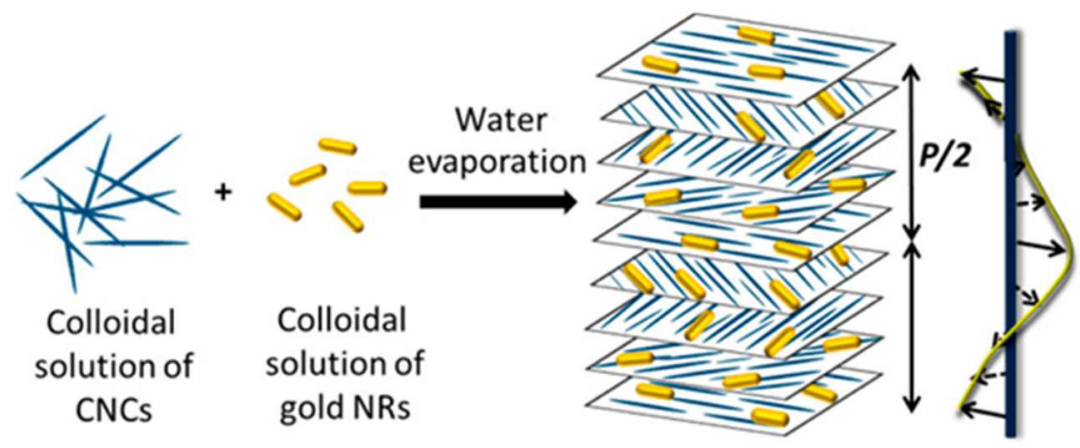

C

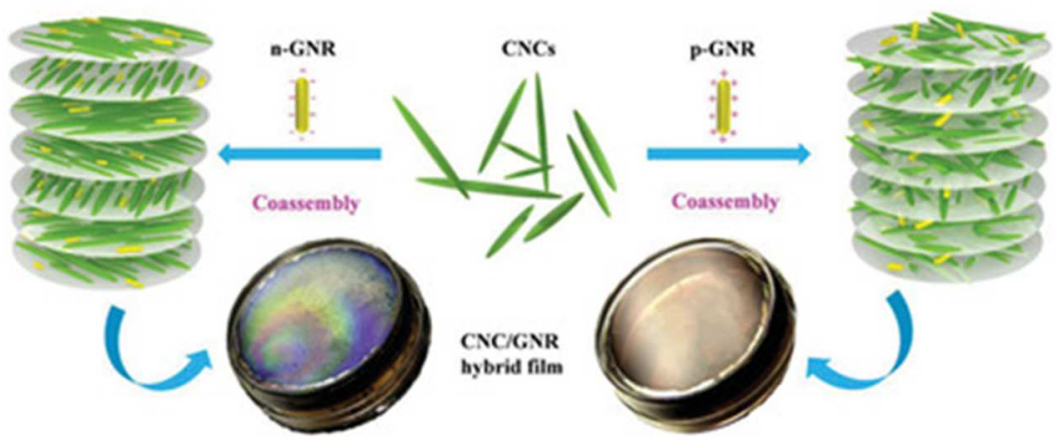


Figure 7. a) Synthesis of chiral nematic mesoporous carbon. (Reproduced from SHOPSOWITZ et al. ${ }^{101}$ Copyright from WILEY-VCH 2011) b) Coassembly of CNWs and GNRs. (Reproduced from QUEREJETA-FERNANDEZ et al. ${ }^{102}$ Copyright from American Chemical Society 2014) c) Coassembly of CNWs and charged GNRs. (Reproduced with modification from CHENG et al. ${ }^{103}$ Copyright from WILEY-VCH 2019)

Coassembly of CNWs and different types of nanoparticles (NPs) is a promising approach for the preparation of multicomponent nanostructured materials with new or enhanced properties. Shopsowitz et al. used the CNWs, extracted from bleached kraft softwood pulp by sulphuric acid hydrolysis, as a chiral nematic template for mesoporous silica. ${ }^{97}$ Mesoporous silicon films with long-range chiral nematic ordering were obtained after the removal of cellulose template by calcination the composite films at $540{ }^{\circ} \mathrm{C}$ under air. The peak reflected wavelength of these obtained films can be varied across the entire visible spectrum and into the near-infrared through simple changes in the synthetic conditions. These chiral mesoporous silica films can be used as hosts to organize particles, such as AgNPs, AuNPs and $\mathrm{TiO}_{2} .{ }^{98-100}$ They also reported that CNW-Silica composite films can be used to generate mesoporous carbon with a high specific surface area and excellent retention of the chiral nematic organization. ${ }^{101}$ After pyrolysis and etching of the silica, free standing films of chiral nematic mesoporous carbon were obtained (Figure 7a). These new carbon films with chiral nematic order may find applications in energy-storage devices, new composite materials, catalyst supports, adsorption media and so on.

The hybrid chiral nematic liquid crystal films show strong plasmonic optical activities can be fabricated by dispersing gold nanoparticles (AuNPs) in CNWs host medium followed by evaporation induced self-assembly process (Figure $7 b$ ). ${ }^{102}$ This is mainly due to the photonic properties of the CNWs host and the plasmonic properties of AuNPs. Furthermore, plasmonic chiroptical properties of the composite films can be tuned by changing the conditions of film preparation. Cheng et al. studied the effect of surface charge and concentration of the gold nanorods (GNRs) on the structure and optical properties of the CNW/GNR films (Figure 7c). ${ }^{103}$ The negatively charged GNRs distribute uniformly in the layered CNWs host, and cause strong electrostatic repulsion among the CNWs resulting in a larger helical pitch than the case without GNRs. The chiral nematic structure of the CNWs preserved well in the solid 
films. However, the positively charged GNRs decrease the chiroptical activity in the composite films due to the electrostatic interaction and particle flocculation.

The coassembly of CNWs and silver nanowires (AgNWs) can also be used to fabricate hybrid chiral nematic liquid crystal films with strong plasmonic optical activities. Chu and coauthors showed that the coassembly process can be tuned by changing the interparticle electrostatic repulsion between the CNWs and AgNWs, two negatively charged particles (Figure 8a). ${ }^{104}$ The guest AgNWs spontaneously form a left-handed chiral distribution when the electrostatic repulsion among CNWs is small. As these repulsive interactions increase, the distribution of AgNWs transform from left-handed to right-handed to minimize the total free energy in the system.

a

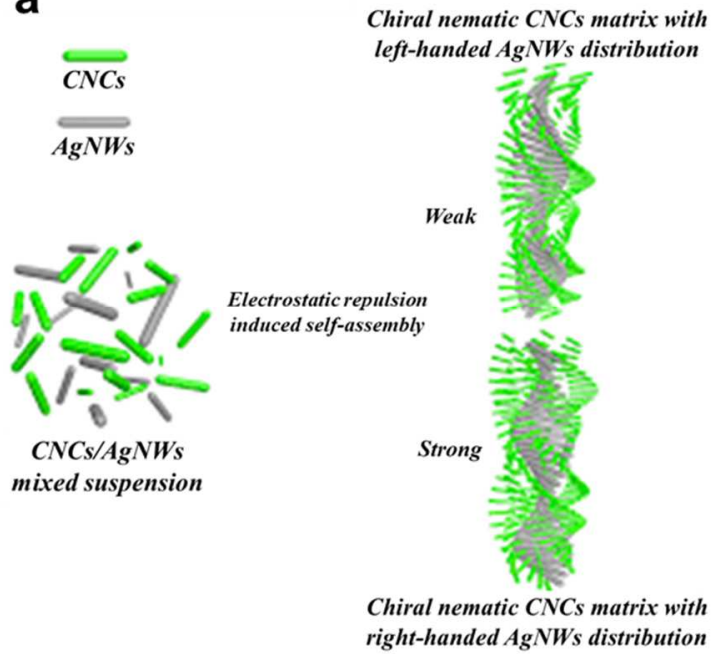

b
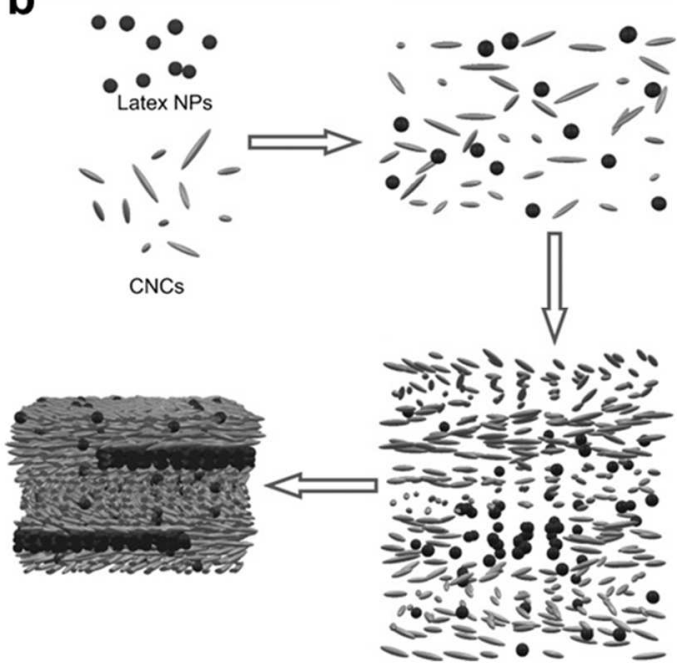

Figure 8. a) The electrostatic interactions between CNWs and AgNWs were exploited to produce helical assemblies with chirality control. (Reproduced with modification from $\mathrm{CHU}$ et al. ${ }^{104}$ Copyright from American Chemical Society 2015) b) Schematic illustration of the coassembly of CNW-latex NP suspensions and films. (Reproduced with modification from THERIEN-AUBIN et al. ${ }^{105}$ Copyright from WILEY-VCH 2015)

The structural characterization of the coassembled structures of CNWs and spherical NPs have also been reported by Thérien-Aubin and coauthors. ${ }^{105}$ They studied the coassembly of CNWs and spherical latex NPs in aqueous suspensions and in solid films. Two phases, chiral nematic CNW-rich phase and isotropic latex-NP-rich phase, exhibit in the mixed CNW-NP 
suspensions (Figure 8b). At the same time, a significant amount of latex NPs portion in the anisotropic chiral nematic domains. After drying, these suspensions form films with planar disordered layers of NPs, which alternated with chiral nematic CNW-rich regions. In addition, NPs were embedded in the chiral nematic domains. The layered morphology of the hybrid films and the random distribution of latex NPs in the anisotropic phase resulted in the films with close-to-uniform fluorescence, birefringence, and circular dichroism properties. In another work, they investigate the effect of the variation in fluorescent latex nanoparticles concentration, size, surface charge, and glass transition temperature on film structure and properties. ${ }^{106}$ They found that small, negatively charged low-glass transition temperature latex particles are the best candidates for the preparation of CNW-latex films with a preserved chiral nematic structure.

The dispersion state of the nanoparticles can also affect the coassembly of CNWs with other nanoparticles. For example, two distinct GO/CNW hybrid films with various internal structures have been obtained via vacuum-assisted self-assembly structure by simply controlling the dispersibility GO in CNWs aqueous solution. ${ }^{107}$ The metastable dispersion resulted $\mathrm{GO} / \mathrm{CNW}$ films with iridescent properties. In contrast, only homogenous mixing of $\mathrm{CNW}$ rod and GO sheet at nanoscale obtained when the dispersion was stable. However, the conductivity of the latter is much better than that of the former. 


\section{Objective of the study}

CNWs as a kind of rod-like nanomaterials have attracted much attention. Additionally, the self-assembly of CNWs and their potential applications for the fabrication of nanocomposites and interaction with other nanoparticles have been extensively studied, while the most commonly used dispersion media are aqueous solutions, which restricts the interaction between homogeneously dispersed CNWs and hydrophobic polymer matrices in non-polar solvents. Thus, surface modifications are often required before suspending in an organic solvent or compounding with hydrophobic substances.

Therefore, the objective of the present study was to create one-step pathways towards the production of hydrophobic CNWs containing acyl moieties that disperse well in non-polar media. The other objective was to explore the self-assembly of hydrophobic surface-acylated CNWs and fabricate functional materials via this method. The main objective of this thesis covers the following points:

1. To investigate the effect of the surface properties of CNW on its self-assembly behaviors and to explore the underlying mechanism of the assembly behaviors.

2. To fabricate novel functional materials from surface-modified CNWs via self-assembly process.

3. To explore the novel properties of the self-assembled materials from CNWs and to give inspirations for the investigation of self-assembly process of other one-dimensional nanomaterials. 


\section{Results and discussion}

\subsection{Self-assembly of CNWs-SU into helical fibers}

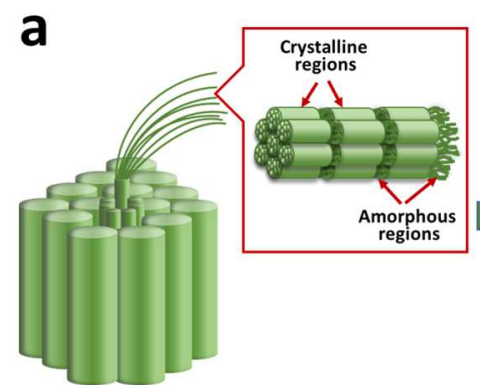

Cellulose

b

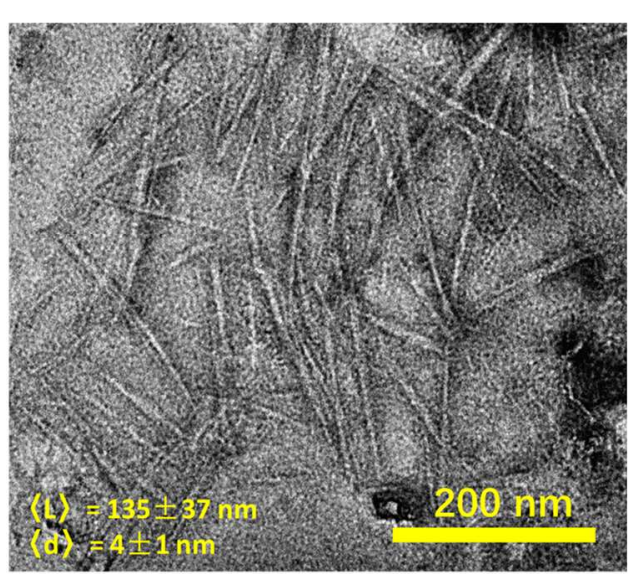

10-undecenoy chloride Pyridine, $50{ }^{\circ} \mathrm{C}, 7 \mathrm{~h}$
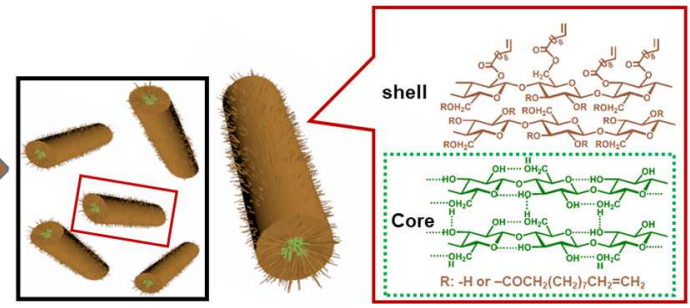

CNWs-SU

C

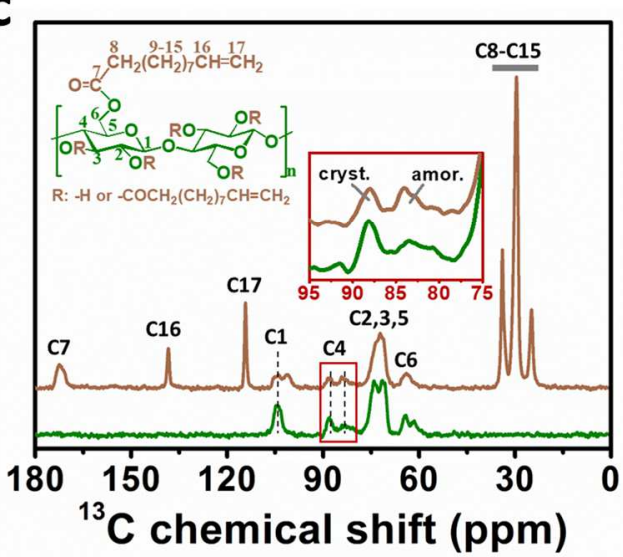

Figure 9. a) Schematic illustration for the synthesis of surface-acylated cellulose nanowhiskers (CNWs-SU). b) A representative TEM image of CNWs-SU1.32. c) Solid-state ${ }^{13} \mathrm{C}$ CP-MAS NMR spectra of microcrystalline cellulose, and CNWs-SU $U_{1.32}$.

As reported in Publication 1, helical fibers with the lengths of micrometers were fabricated via evaporation-driven self-assembly of surface-acylated cellulose nanowhiskers (CNWs-SU) after drying on silicon wafers. CNWs-SU were synthesized from microcrystalline cellulose (MCC) after the surface esterification by 10-undecenoyl chloride under heterogeneous conditions and separated via ultracentrifugation (Figure 9a). The contents of surface-immobilized 10-undecenoyl groups were determined to be on average $3.52 \mathrm{mmol} / \mathrm{g}$ based on the elemental analysis, which corresponds to a degree of substitution (DS) of 1.32. This product is thus referred to as CNWs-SU $\mathrm{CU}_{1.32}$ and they have typical whisker shapes as displayed by TEM images (Figure 9b). They have an average length of $135 \pm 37 \mathrm{~nm}$ 
and an average diameter of $4 \pm 1 \mathrm{~nm}$. Solid-state ${ }^{13} \mathrm{C}$ CP-MAS NMR and FTIR spectra of MCC and $\mathrm{CNWs}-\mathrm{SU}_{1.32}$ further confirmed the chemical compositions and were used for the determination of their crystallinities (Figure 9c). The crystallinity of MCC is determined to be approximately $58 \%$ and that of CNWs-SU 1.32 is about $40 \%$.
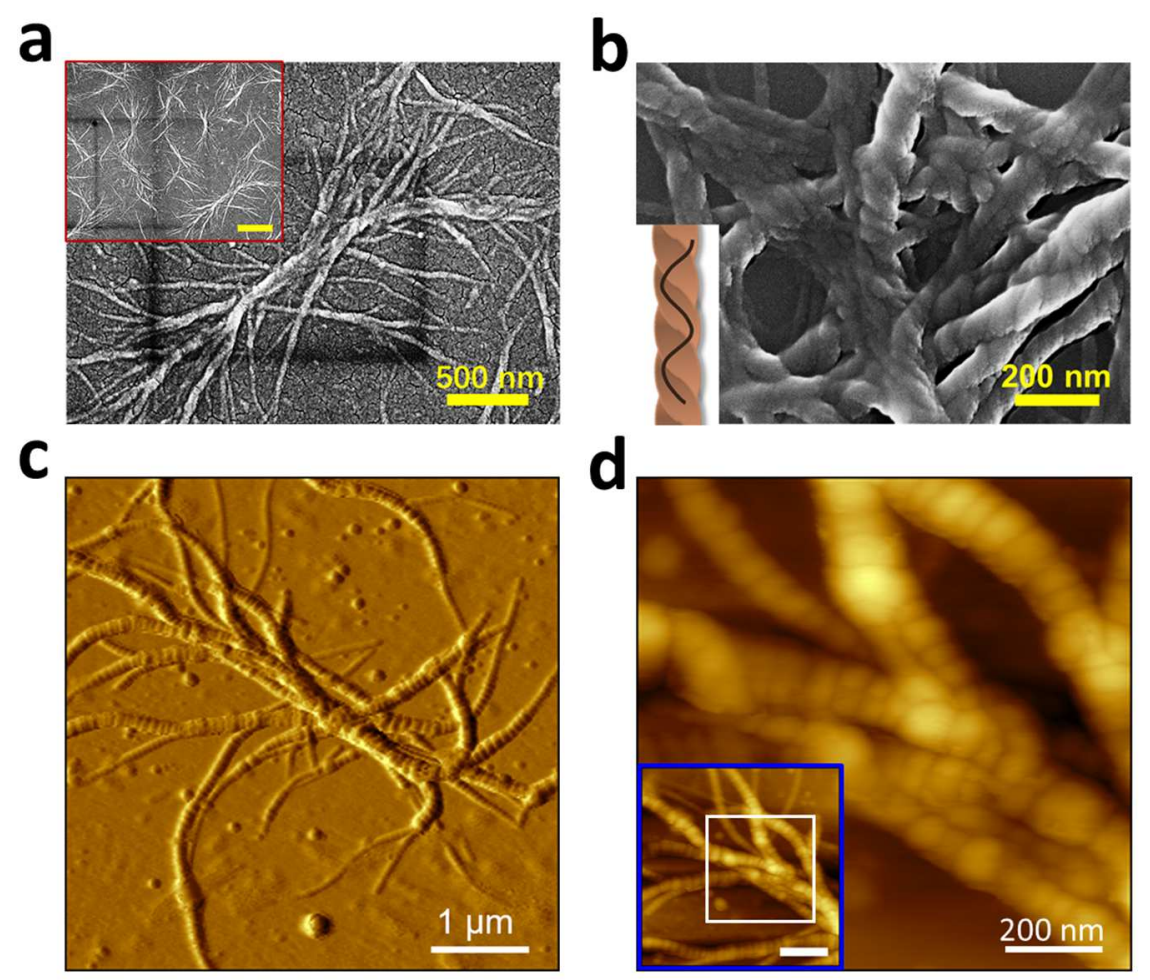

Figure 10. a) A typical SEM image of helical fibers constructed by CNWs-SU1.32. The inset shows more typical helical fibers with the scale bar of $1 \mu \mathrm{m}$. b) A representative SEM image of helical fibers constructed by CNWs-SU1.32 showing details of the helix. The inset shows a schematic illustration of the left-handed helical structure. c) AFM amplitude image of helical fibers formed on silicon wafer using the THF suspension of CNWs-SU $\mathrm{CU}_{1.32}$ at a concentration of $0.0002 \mathrm{mg} / \mathrm{mL}$. d) Magnified regions of the AFM image from (c) showing the detailed helical structures. The scale bar in the inset is $500 \mathrm{~nm}$.

After depositing the THF suspensions of CNWs-SU $\mathrm{SU}_{1.32}$ on the surface of silicon wafers, CNWs-SU 1.32 gradually self-assembled into helical fibers during drying in the air under ambient conditions. These integrated helical structures can reach a length of 5-6 $\mu \mathrm{m}$ and contain smaller fibers with a length between 1-2 $\mu \mathrm{m}$ (Figure 10a,c). The smaller fibers are 
generally twisted and composed of several single $\mathrm{CNWs}_{-} \mathrm{SU}_{1.32}$. Moreover, the helices in these helical fibers are generally left-handed (Figure 10b,d).
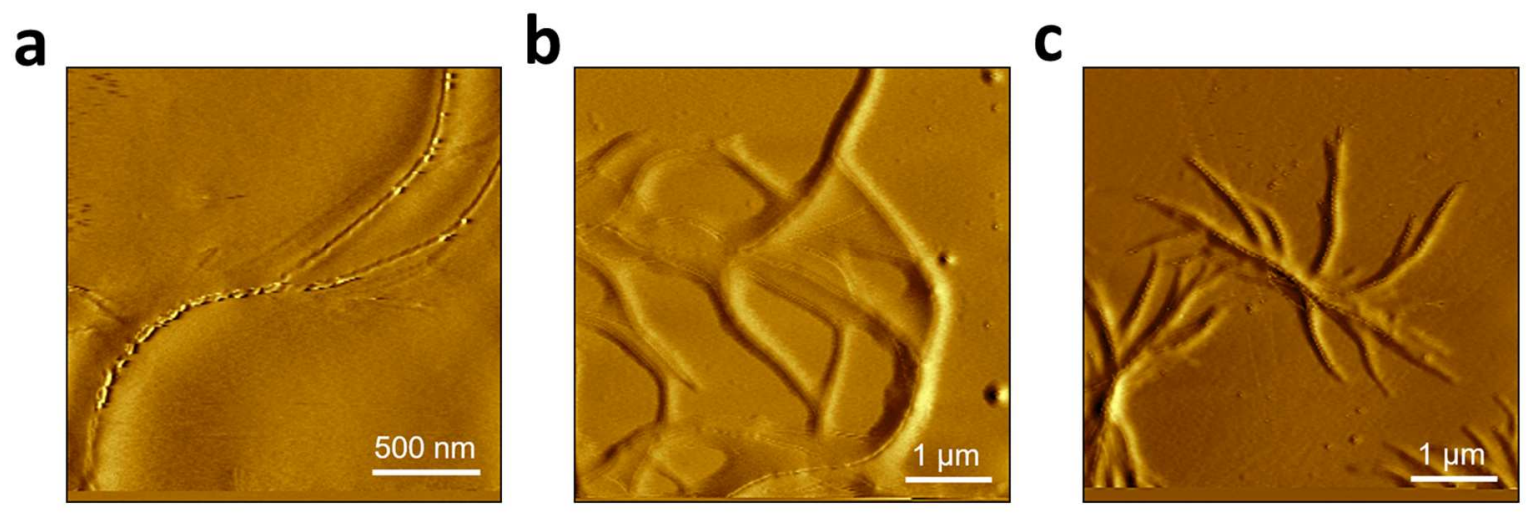

Figure 11. AFM images of (a) $\mathrm{PCUE}_{1.1}$, (b) $\mathrm{CUE}_{3}$, (c) $\mathrm{CSE}_{3}$.

As comparison, a polymeric cellulose 10-undecenoyl ester with a DS of 1.10 (referred to as PCUE 1.1 , polymeric cellulose 10-undecenoyl ester and cellulose stearoyl ester with complete derivatization of hydroxy groups and thus DS of 3 (referred to as $\mathrm{CUE}_{3}$ and $\mathrm{CSE}_{3}$ ), did not form helical fibers under similar conditions (Figure 11). Thus, the presence of rigid CNWs as the crystalline core is a determining factor for the formation of helical fibers. 

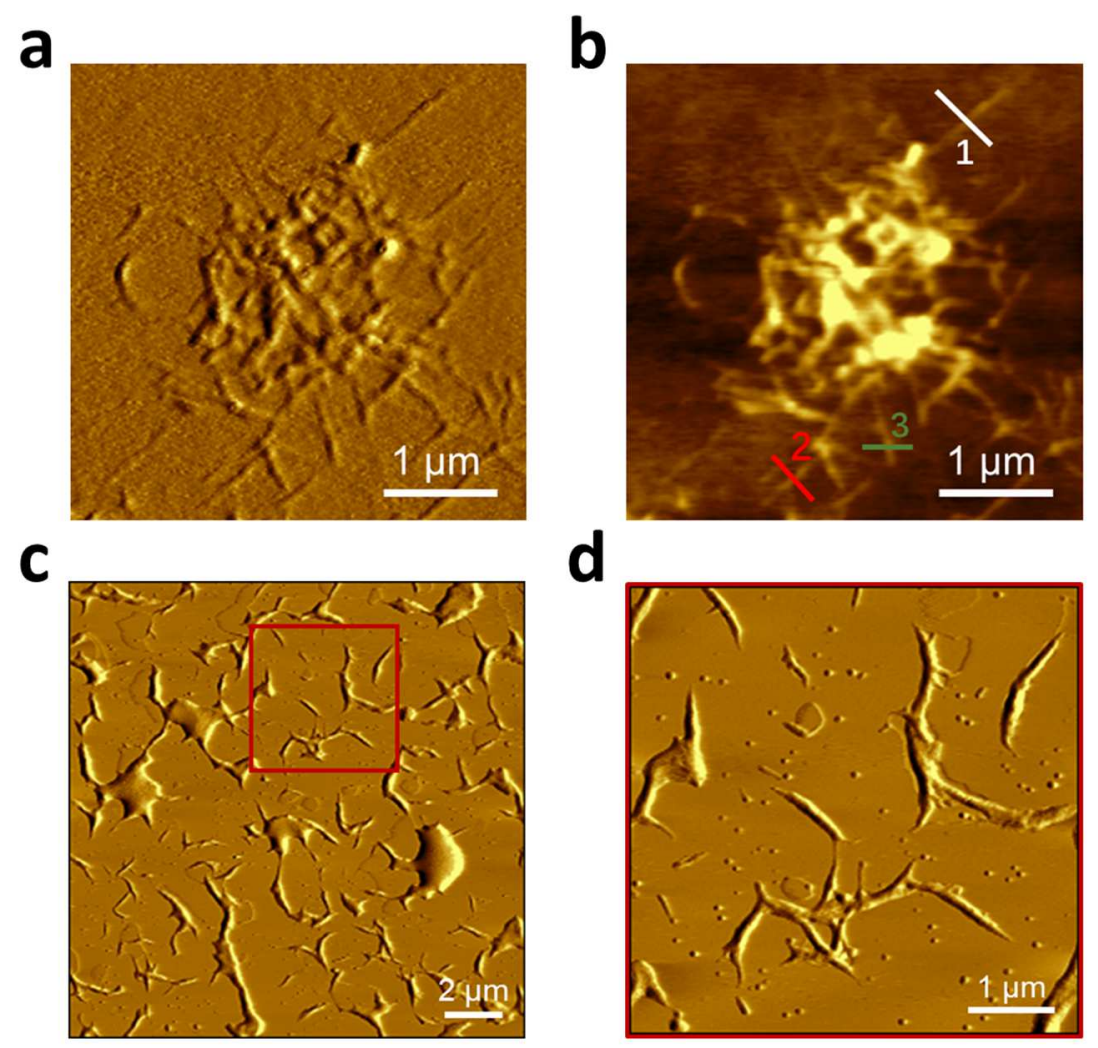

Figure 12. AFM (a) amplitude and (b) height image of dried $\mathrm{CNWs}_{-} \mathrm{SU}_{1.32}$ on PDMS substrate using their THF suspensions at a concentration of $0.0002 \mathrm{mg} / \mathrm{mL}$. c) AFM image of dried CNWs-SU $\mathrm{SU}_{1.32}$ on the surface of hydrophobized silicon wafer using their THF suspensions at a concentration of $0.0002 \mathrm{mg} / \mathrm{mL}$. d) AFM image of the magnified region as in (c).

In contrast to the helical structures on the silicon wafers, CNWs-SU ${ }_{1.32}$ did not form helical fibers on the surface of PDMS, but only aggregates (Figure 12a,b). In a similar way, no helical fiber was generated by CNWs-SU1.32 on the surface of hydrophobized silicon wafer. Instead, CNWs-SU1.32 tended to aggregate into smooth fibers (Figure 12a,c). Therefore, CNWs-SU 1.32 cannot self-assemble into helical structures on hydrophobic substrates under applied conditions. This is primarily due to the presence of surface-attached non-polar acyl chains. CNWs-SU1.32 should spread better on hydrophobic surfaces via the hydrophobic interaction between surface-attached acyl chains and hydrophobic surface. In comparison, CNWs-SU $U_{1.32}$ prefer to self-assembly with each other on hydrophilic surfaces. 

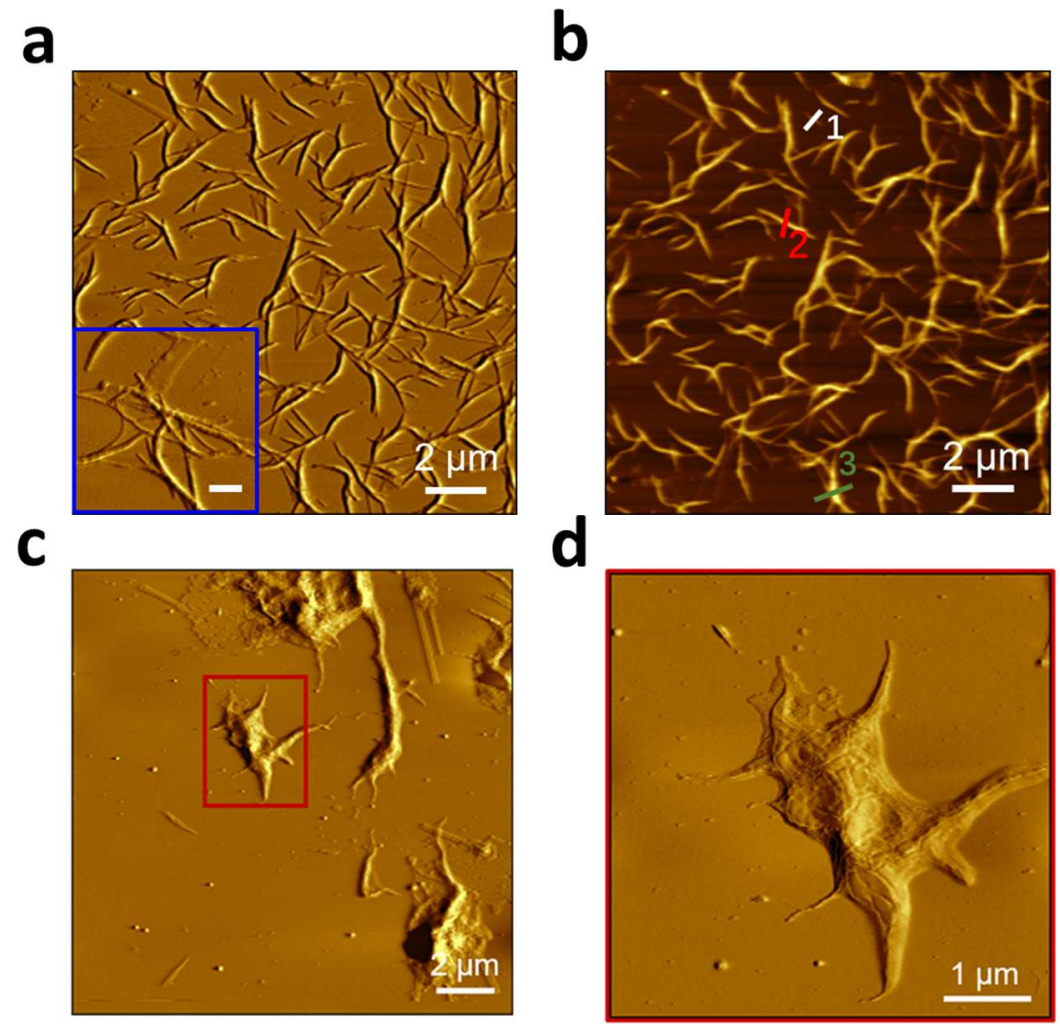

Figure 13. AFM (a) amplitude and (b) height image of dried CNWs-SU $\mathrm{CU}_{1.32}$ on silicon wafer using their DCM suspensions at a concentration of $0.0002 \mathrm{mg} / \mathrm{mL}$. The scale bar in the inset is $500 \mathrm{~nm}$. c) AFM image of dried CNWs-SU 1.32 on silicon wafer using their toluene suspensions at a concentration of $0.0002 \mathrm{mg} / \mathrm{mL}$. d) AFM image of the magnified region as in (c).

Different structures were obtained by using distinct solvents, that is, THF, DCM and toluene, which demonstrates the critical effect of solvent evaporation on the self-assembly process (Figure 13). THF with an intermediate evaporation rate under atmospheric conditions provided sufficient time for $\mathrm{CNWs}-\mathrm{SU}_{1.32}$ to self-assemble into helical fibers. In comparison, faster evaporation of DCM did not assure sufficient time for CNWs-SU $\mathrm{CU}_{1.32}$ to self-assembly, while only single short fibers of aggregated CNWs-SU $\mathrm{SU}_{1.32}$ were obtained. Toluene with a much lower evaporation rate only led to strong aggregation due to the continuous accumulation of CNWs-SU1.32. 


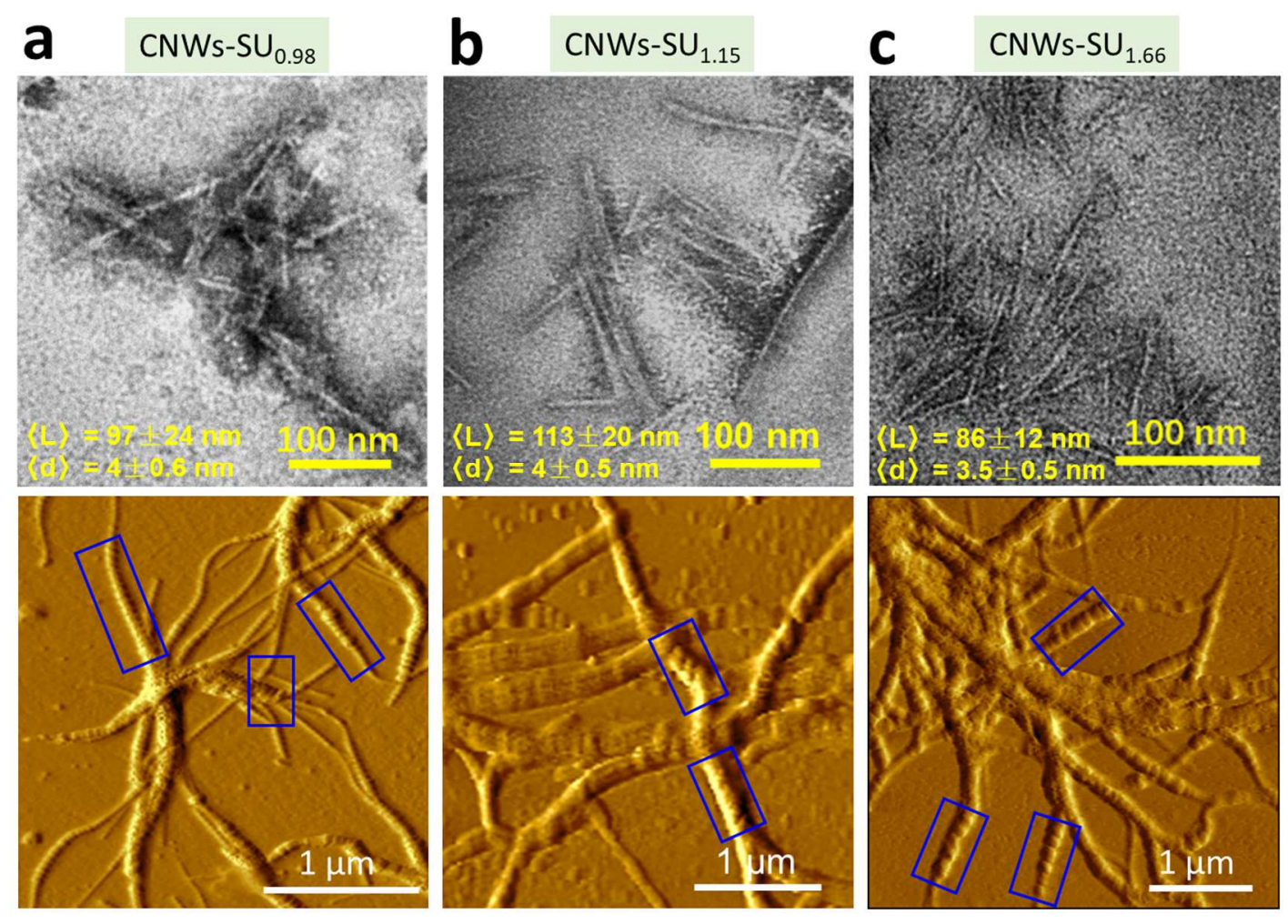

Figure 14. Representative TEM images and AFM amplitude images of diverse CNWs-SU on silicon wafers using their THF suspensions at a concentration of $0.0002 \mathrm{mg} / \mathrm{mL}$ : (a) CNWs$\mathrm{SU}_{0.98}$, (b) CNWs-SU1.15, and (c) CNWs-SU1.66.

The DSs of diverse CNWs-SU play a critical role in the generation of explicit left-handed helical fibers via self-assembly, while the crystallinity of diverse CNWs-SU affects the formation of helical fibers to a certain extent due to their association with the alternating DSs during the reaction. Too low or too high amounts of surface-attached 10-undecenoyl groups are not preferable for the self-assembly of helical fibers, while an intermediate amount of undecenoyl groups is more suitable (Figure 14). 

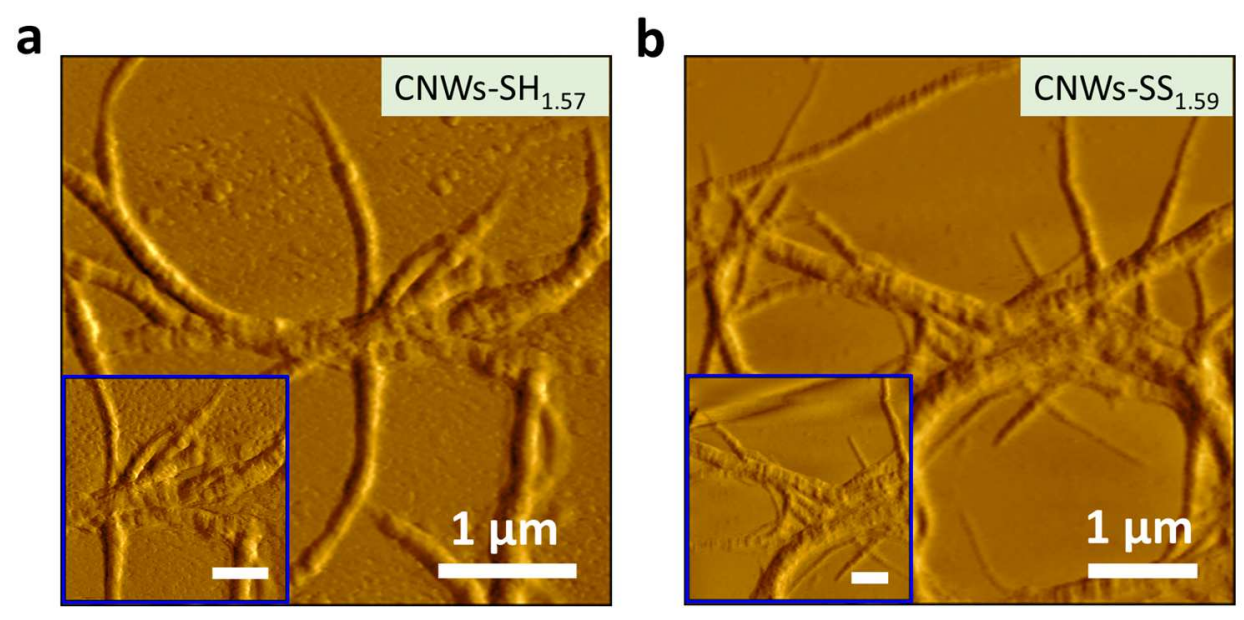

Figure 15. a) AFM amplitude image of $\mathrm{CNWs} \mathrm{SH}_{1.57}$ dried on silicon wafers using its $\mathrm{THF}$ suspension at a concentration of $0.0002 \mathrm{mg} / \mathrm{mL}$. The scale bar in the inset is $500 \mathrm{~nm}$. b) AFM amplitude image of CNWs-SS 1.59 dried on silicon wafers using its THF suspension at a concentration of $0.0002 \mathrm{mg} / \mathrm{mL}$. The scale bars in the inset are $500 \mathrm{~nm}$.

CNWs with surface-attached hexanoyl groups $\left(\mathrm{CNWs}-\mathrm{SH}_{1.57}\right)$ or stearoyl groups (CNWs$\mathrm{SS}_{1.59}$ ) were synthesized correspondingly. Both $\mathrm{CNWs}_{-} \mathrm{SH}_{1.57}$ and $\mathrm{CNWs}-\mathrm{SS}_{1.59}$ could not self-assemble into significant helical fibers, but only formed long fibers with periodic rough surface patterns during the drying process (Figure 15). Thus, the acyl chain lengths strongly influence the formation of helical fibers, while the 10-undecenoyl groups are the most appropriate acyl chains compared to hexanoyl and stearoyl moieties.

In conclusion, we showed the formation of helical fibers with the lengths of micrometers by the evaporation-driven self-assembly on silicon wafers of functionalized CNWs with surface-attached acyl chains. The self-assembly process and the final helical structures were affected by parameters including the wettability of substrates, dispersing solvents, the amount of 10-undecenoyl groups, the crystallinity, the dimension of CNWs, and the length of acyl chains. 


\subsection{Self-assembly of surface-acylated CNWs for Janus films}
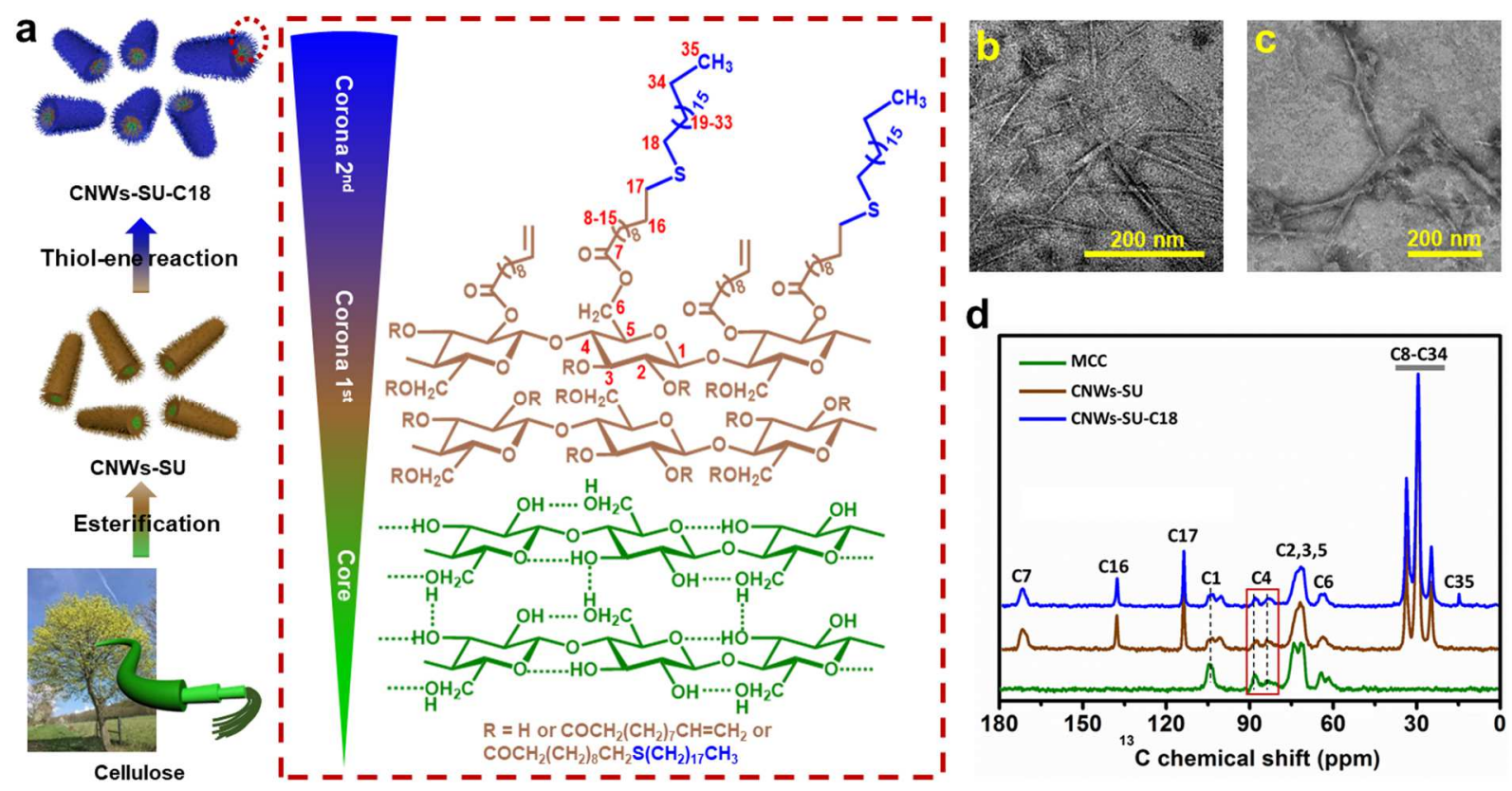

Figure 16. Synthesis and characterization of surface-acylated CNWs. a) Schematic illustration for the synthesis of CNWs-SU and CNWs-SU-C18. b) A representative TEM image of CNWs-SU. c) A representative TEM image of CNWs-SU-C18. d) Solid-state ${ }^{13} \mathrm{C}$ CP-MAS NMR spectra of microcrystalline cellulose, CNWs-SU and CNWs-SU-C18.

As demonstrated in Publication 1, surface-acylated CNWs were obtained after surface esterification of MCC by 10-unddecenoyl chloride under heterogeneous conditions. The presence of reactive terminal double bonds in surface-acylated CNWs allows further modifications. In publication 2, CNWs-SU were further modified through the thiol-ene reaction of the terminal double bonds with 1-octadecanethiol to synthesis CNWs-SU-C18 (Figure 16a). Compared with CNWs-SU, CNWs-SU-C18 still maintained their original sizes after the introduction of further alkane moieties (Figure 16b,c). For instance, CNWs-SU$\mathrm{C} 18 / 75$ had an average dimension of $146 \pm 33 \mathrm{~nm}$ in length and $4 \pm 1 \mathrm{~nm}$ in diameter. Compared with the ${ }^{13} \mathrm{C}$ CP-MAS NMR spectra of CNWs-SU, a new signal emerged at 15 ppm (C35) and is ascribed to the terminal methyl carbons of the long-chain hydrocarbons, which suggests the successful introduction of thiols via the thiol-ene reaction for CNWs-SU-C18 (Figure 16d). The crystallinity of CNWs-SU-C18/75 was determined to be about $33 \%$ according to the ${ }^{13} \mathrm{C}$ 
CP-MAS NMR spectra. Based on the elemental analysis, the content of 1-octadecane groups in CNWs-SU-C18 was determined to be $1.37 \mathrm{mmol} / \mathrm{g}$.

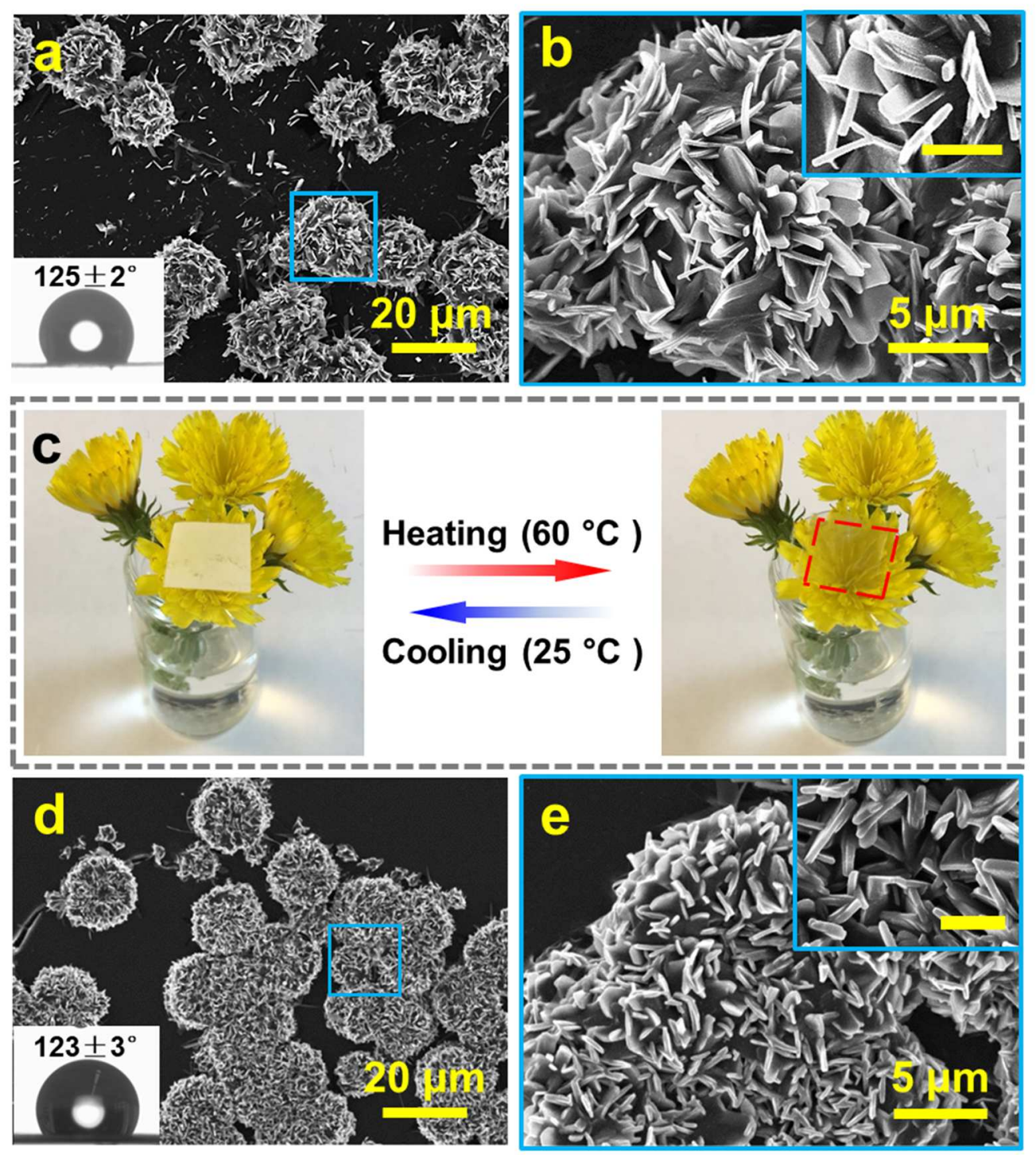

Figure 17. (a) Representative SEM image of the top surface of the CNWs-SU-C18/75 film. The inset shows static water contact angle (SWCA) on the top surface. (b) SEM image of the magnified region as in (a). The inset shows the magnified particle surface in (b) with the scale bar of $2 \mu \mathrm{m}$. c) Photo images of one CNWs-SU-C18/75 film on flowers at 25 and $60{ }^{\circ} \mathrm{C}$. d) Representative SEM image of the CNWs-SU-C18/75 film after heat-treatment at $60{ }^{\circ} \mathrm{C}$, cooling down to room temperature and further aging at room temperature for more than two days. The inset shows SWCA on this surface. e) SEM image of the magnified region as in (d). The inset shows the magnified particle surface in (e) with the scale bar of $2 \mu \mathrm{m}$. 
Solvent-casting THF suspensions of CNWs-SU-C18/75 on Teflon substrates resulted in self-standing films. The top surface of as-prepared CNWs-SU-C18/75 films contained hierarchical micro/nanostructure. Many spherical microscale particles were randomly distributed on the top surface of CNWs-SU-C18/75 films (Figure 17a). Figure 17b shows a high-resolution SEM image of a single particle, where lamellar nanoplates with an average thickness of $196 \pm 36 \mathrm{~nm}$ are vertically present on the whole surface of microparticles. This self-assembled hierarchical structure on the top surface led to highly hydrophobic surfaces. In comparison, no hierarchical micro/nanostructure formed on the bottom surface of the film, which causes the surface with lower hydrophobicity.

Furthermore, the non-transparent CNWs-SU-C18/75 films turned transparent after the treatment at $60{ }^{\circ} \mathrm{C}$ within one minute due to the disappearance of microparticles (Figure 17c). When the temperature was lowered to room temperature, the films became non-transparent again due to the formation of microparticles. The cooling down from $60{ }^{\circ} \mathrm{C}$ to room temperature took less than $2 \mathrm{~min}$, while it took a few further days for the films to become nontransparent again. During the cooling process, the lamellar nanoplates on spherical microparticles were gradually reformed (Figure 17d,e).
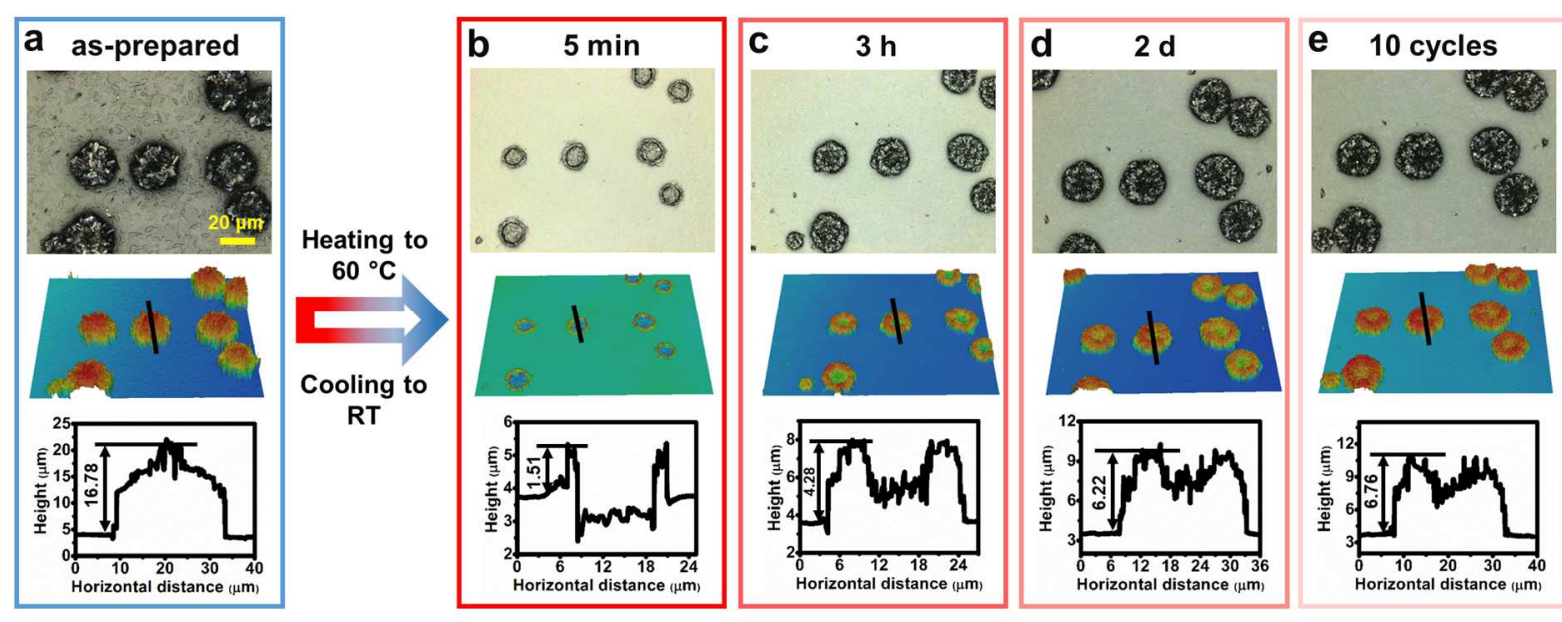

Figure 18. Thermo-reversible properties of CNWs-SU-C18/75 film. a) Representative laser scanning microscope (LSM) image, 3D LSM image and height profile of microparticles on the top surface of a CNWs-SU-C18/75 film. b-d) Time-dependent LSM images, 3D LSM images, and height profiles of the self-assembled structures after cooling down from $60^{\circ} \mathrm{C}$ to room temperature and further aging time of $5 \mathrm{~min}, 3 \mathrm{~h}$, and $2 \mathrm{~d}$, respectively. e) LSM image, 3D LSM image, and height profile of the CNWs-SU-C18/75 film after 10 cycles of heating- 
cooling process. The images and height profile after the $10^{\text {th }}$ cycle were recorded after maintaining at room temperature for 2 days. RT: room temperature.

Remarkably, the LSM measurement also reveals that these self-assembled structures exhibited thermo-reversible properties (Figure 18). Although these donut-like microparticles are different from the original spheres in the as-prepared films, they can be reconstructed during the following heating-cooling cycles with constant thermo-reversible properties. No significant fatigue of the morphology was observed after 10 cycles except for the slightly variable outside diameters of the donuts. The regeneration of such self-assembled donut-like particles was assumed to be realized via two steps: melting and recrystallization. The observation that the outside diameter of the donuts remained unaltered by multiple heatingcooling cycles suggested that the contact line of the molten material was pinned at the surface of Janus films. After the first heating, the molten material densified, i.e., the volume of the molten drop was smaller than that of the original sphere. This volume shrinkage in conjunction with the pinned contact line resulted in the formation of a molten rim. After rapid cooling down to room temperature and aging, the molten rim crystallizes, leading to the donut structures. The regeneration of such microsized particles leads to the significant transition of the surface topography, which dominates the recovery of the nontransparency and hydrophobicity of CNWs-SU-C18/75 films. 

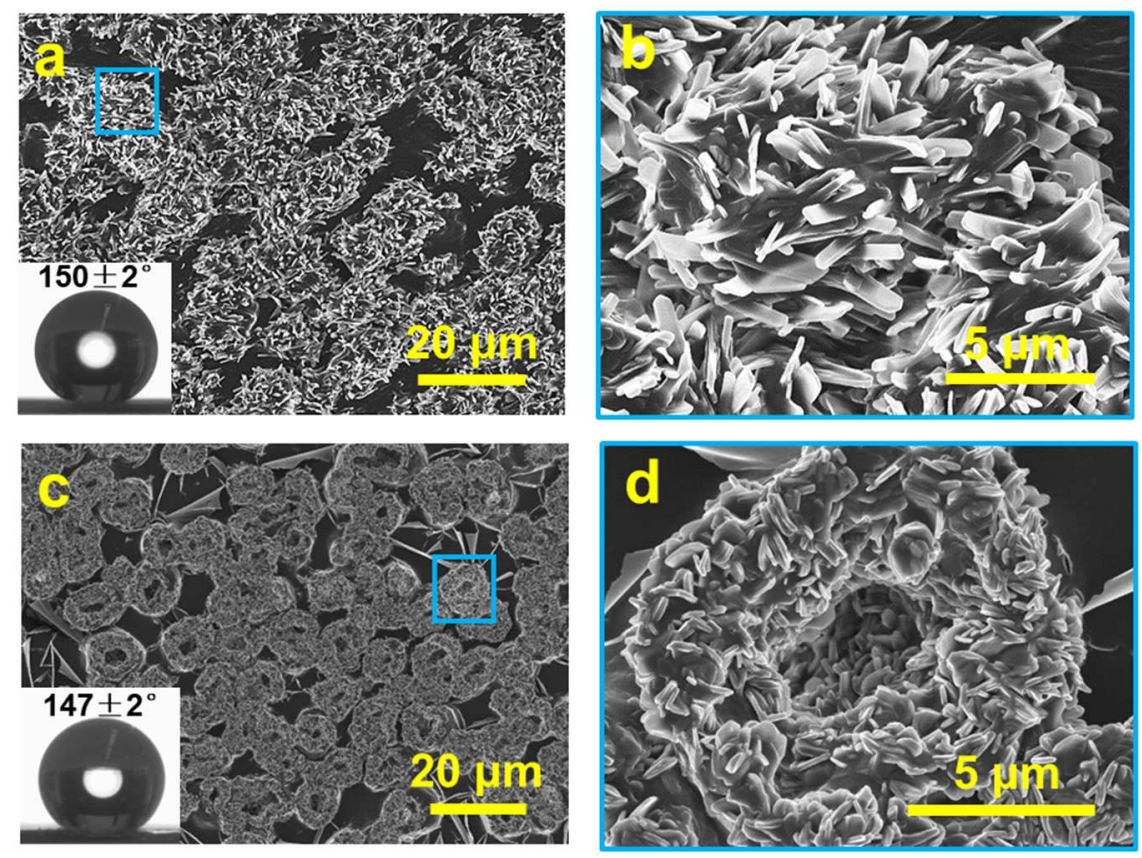

Figure 19. a) Representative SEM image of the top surface of a CNWs-SU-C18/75+GO(4:1) film. The inset shows SWCA on the top surface. b) SEM image for the magnified region as in (a). c) Representative SEM image of the top surface of CNWs-SU-C18/75+GO(4:1) film after heating at $60{ }^{\circ} \mathrm{C}$, cooling down to room temperature and maintaining at room temperature for more than two days. The inset shows SWCA on the surface. d) SEM image for the magnified region as in (c).

To further adjust the morphology and the surface properties of obtained films, GO with a different surface wettability was added in the suspensions of CNWs-SU-C18/75 to prepare Janus films. Compared with the self-assembly of solely CNWs-SU-C18/75, the coassembly of CNWs-SU-C18/75 and GO resulted in more spherical structures formed on the top surface of films, which should be attributed to the heterogeneous nucleation of CNWs-SU-C18 in bulk suspensions where the GO act as heterogeneous nuclei (Figure 19a,b). Furthermore, the Janus films of CNWs-SU-C18/75+GO(4:1) also exhibited thermo-reversible property. The spherical structures on the top surface disappeared after heat-treatment at $60{ }^{\circ} \mathrm{C}$ and recovered as flat donut-like particles after cooling down to room temperature (Figure 19c). After such treatments, the middle part of donut-like particle collapsed and formed a circular structure with lamellar nanoplates on the surface (Figure 19d). 


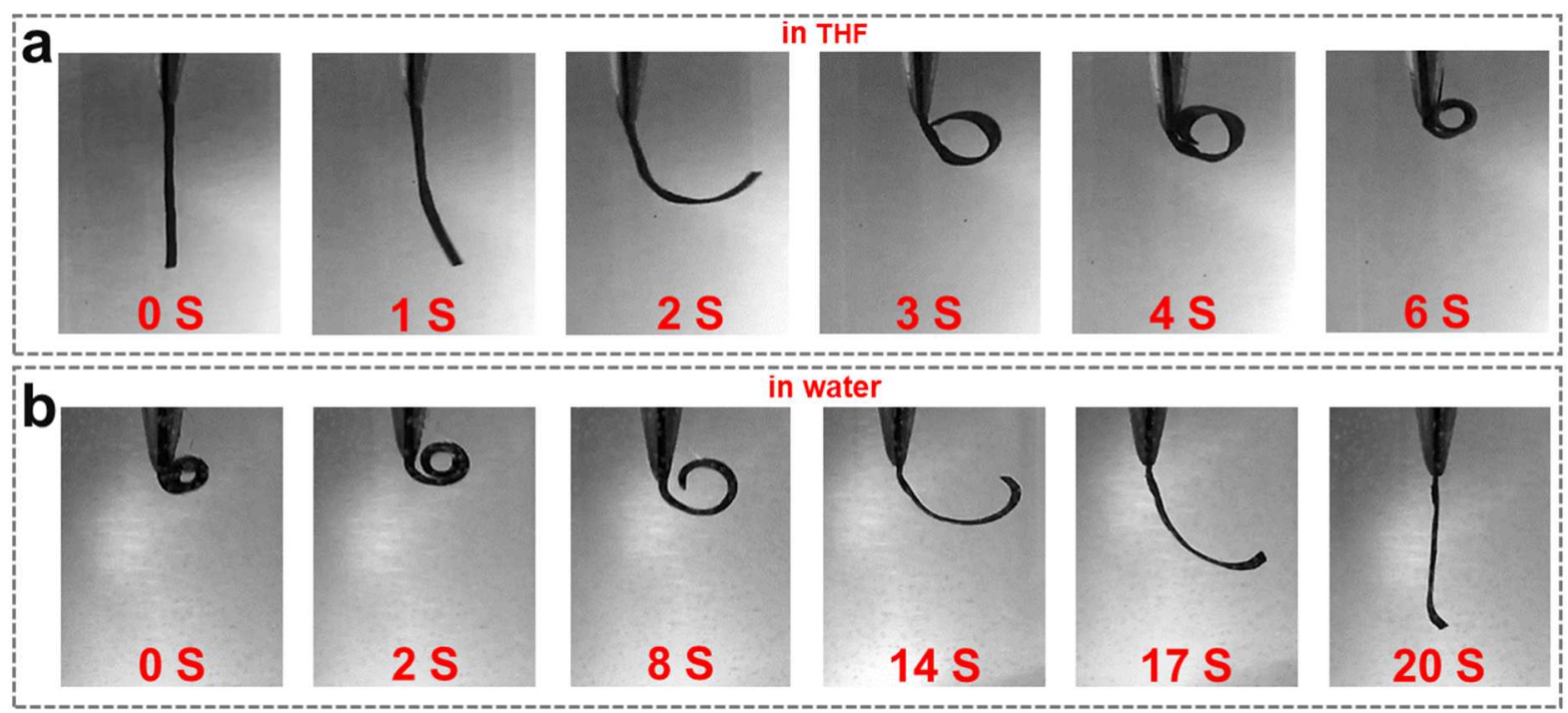

Figure 20. a, b) Snapshots showing reversible bending processes of the Janus film stripes in (a) THF after 0, 1, 2, 3, 4 and $6 \mathrm{~s}$ as well as (b) water after 0, 2, 8, 14, 17 and $20 \mathrm{~s}$.

In addition to the thermo-reversible behaviors of the micro/nano-structures on the top surface, these Janus films also exhibited solvent-responsive properties. As soon as the stripes were placed in the THF solution, the straight films instantly rolled up into highly curled films. This process was very fast with rapidly increasing bending angles and finished within $6 \mathrm{~s}$ (Figure 20a). When the curled stripes immersed into water, they responded by opening up with decreasing bending angles and became straight within $20 \mathrm{~s}$ (Figure 20b). This is mainly due to the asymmetric structures in the Janus films and distinct swelling behaviors of CNWs and GO led to curling and flattening behaviors of these hybrid Janus films in diverse solvents. The Janus films maintained the fast bending performance even after 25 runs, by alternatively placing them in THF and water under ambient conditions.

In conclusion, we developed a facile one-step method to fabricate multiresponsive Janus films with reconstructible time-dependent micro-/nanostructures and asymmetric wettability via the evaporation-driven self-assembly of surface-acylated CNWs bearing long alkane chains with or without GO. 


\subsection{Self-assembly of CNWs-SU confined in honeycomb films}
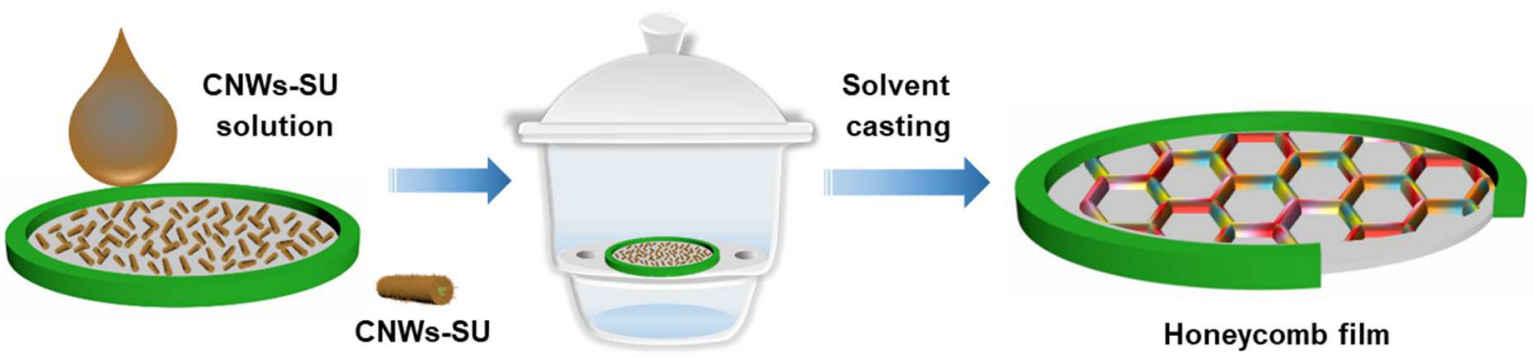

Figure 21. Schematic illustration for the fabrication of CNWs-SU honeycomb film with breath figure method.

As demonstrated in Publication 1, CNWs with surface-immobilized 10-undecenoyl groups (CNWs-SU) were synthesized from MCC after the surface esterification by 10-undecenoyl chloride. CNWs-SU honeycomb films were prepared using a static BF approach in water saturated atmosphere (Figure 21). $10 \mathrm{~mL}$ of a DCM solution of CNWs-SU (3 mg/mL) was cast on Teflon mould and kept at room temperature with a relative humidity of $75 \%$. 

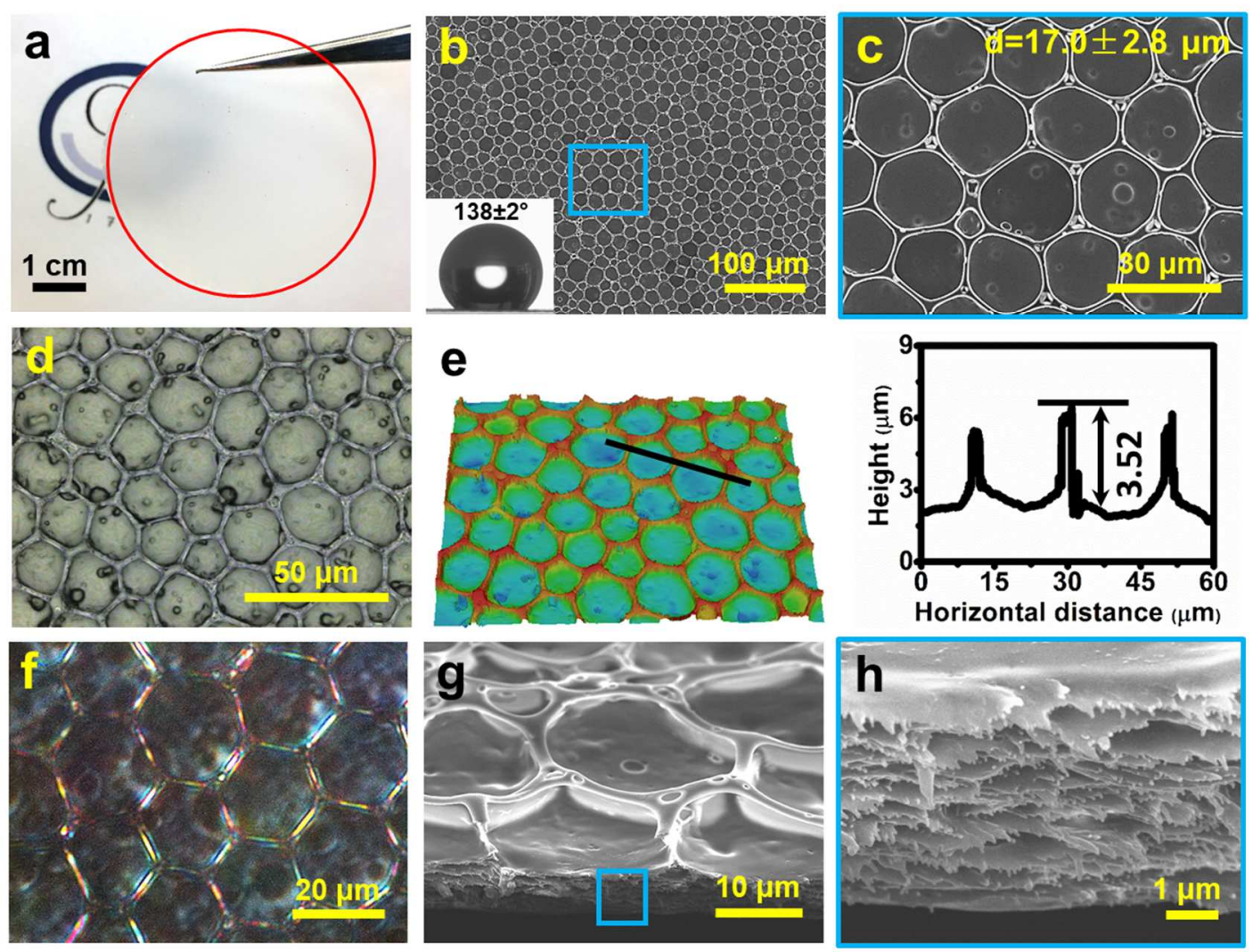

Figure 22. a) Photo image of the dried honeycomb film fabricated with CNWs-SU. b) Representative SEM image of the top surface of the honeycomb film fabricated with CNWsSU. The inset shows SWCA on the top surface. c) SEM image of the magnified region as in (b). d) Representative LSM image of the honeycomb film fabricated with CNWs-SU. e) 3D LSM image and height profile of the honeycomb film fabricated with CNWs-SU. f) PLM image of the honeycomb film fabricated with CNWs-SU. g) Representative SEM image of the cross section of the honeycomb film fabricated with CNWs-SU. h) SEM image of the magnified region as in $(\mathrm{g})$.

After complete evaporation of solvent and water droplets, we obtained honeycomb films with microscale pores on the top surfaces (Figure 22a). The porous surfaces gave rise to a high contact angle of about $138 \pm 2^{\circ}$ due to their structured surfaces (Figure 22b). The SEM image shows that the diameter of pores was around $17.0 \pm 2.8 \mu \mathrm{m}$ and the width of the rims was about $1.8 \pm 0.4 \mu \mathrm{m}$ (Figure 22c). The LSM measurement also reveals that the obtained film exhibited honeycomb-like structures (Figure 22d). Microsized pores uniformly distributed on the top surface of the films. According to the height profile obtained from 3D LSM image, the average depth of the pores was about $3.7 \pm 0.5 \mu \mathrm{m}$ (Figure 22e). It is worth 
pointing out that the rim of the obtained honeycomb film displayed iridescent color as shown in the PLM image (Figure 22f). Ring-shaped iridescent patterns were observed around the pores, but no iridescent color was observed from the interior part of the pore, indicating that the CNWs highly arranged along the boundary of micropores. The SEM cross-sectional view in Figure $22 \mathrm{~g}$ shows the contour of the pores. The depth of the pores was about $3.8 \mu \mathrm{m}$ which consistent with the result of 3D LSM. The height of the underneath diameter was about $10.3 \pm 0.5 \mu \mathrm{m}$. The pore depth and underneath diameter were smaller than the open diameter $(17.0 \pm 2.8 \mu \mathrm{m})$ of the pore on the top surface. Accordingly, the aspect ratio of depth of pores and the height of underneath diameter $\left(\mathrm{r}_{\mathrm{D} / \mathrm{H}}\right)$ was about $35.6 \%$. From the magnified SEM image of the cross section, the CNW film showed a typical layered morphology (Figure 22h).
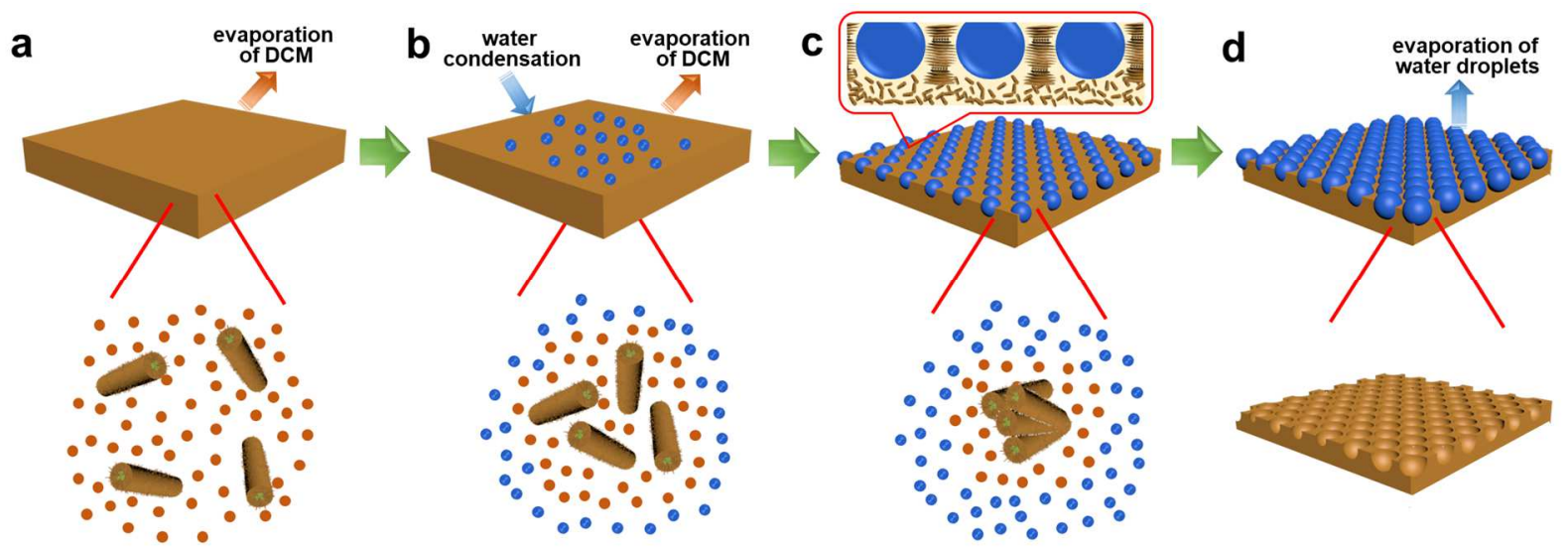

Figure 23. Schematic illustration for the self-assembly process of CNWs-SU into honeycomb films.

Figure 23 presents a schematic explanation as how the ordered porous films form. At first, the CNWs solution spread on the substrate homogeneously (Figure 23a). As the solvent evaporates, leading to the cooling of the solution surface, the surrounding water vapor forms small droplets and deposits on the surface of the solution. The presence of water droplets changes the characteristics of the solvent dramatically: inducing a phase inversion and aggregation of the hydrophobic CNWs (Figure 23b). The continuous condensation of water vapor, causes the growth and self-organization of the water droplets into ordered droplet array and forces the CNWs to move closer together to form aggregates (Figure 23c), thereby induces the formation of walls and pores. The walls, composed of stacked and overlapped 
CNWs, are highly arranged along the boundary of micropores. Complete evaporation of DCM and then the water droplets, the ordered porous films formed (Figure $23 \mathrm{~d}$ ).
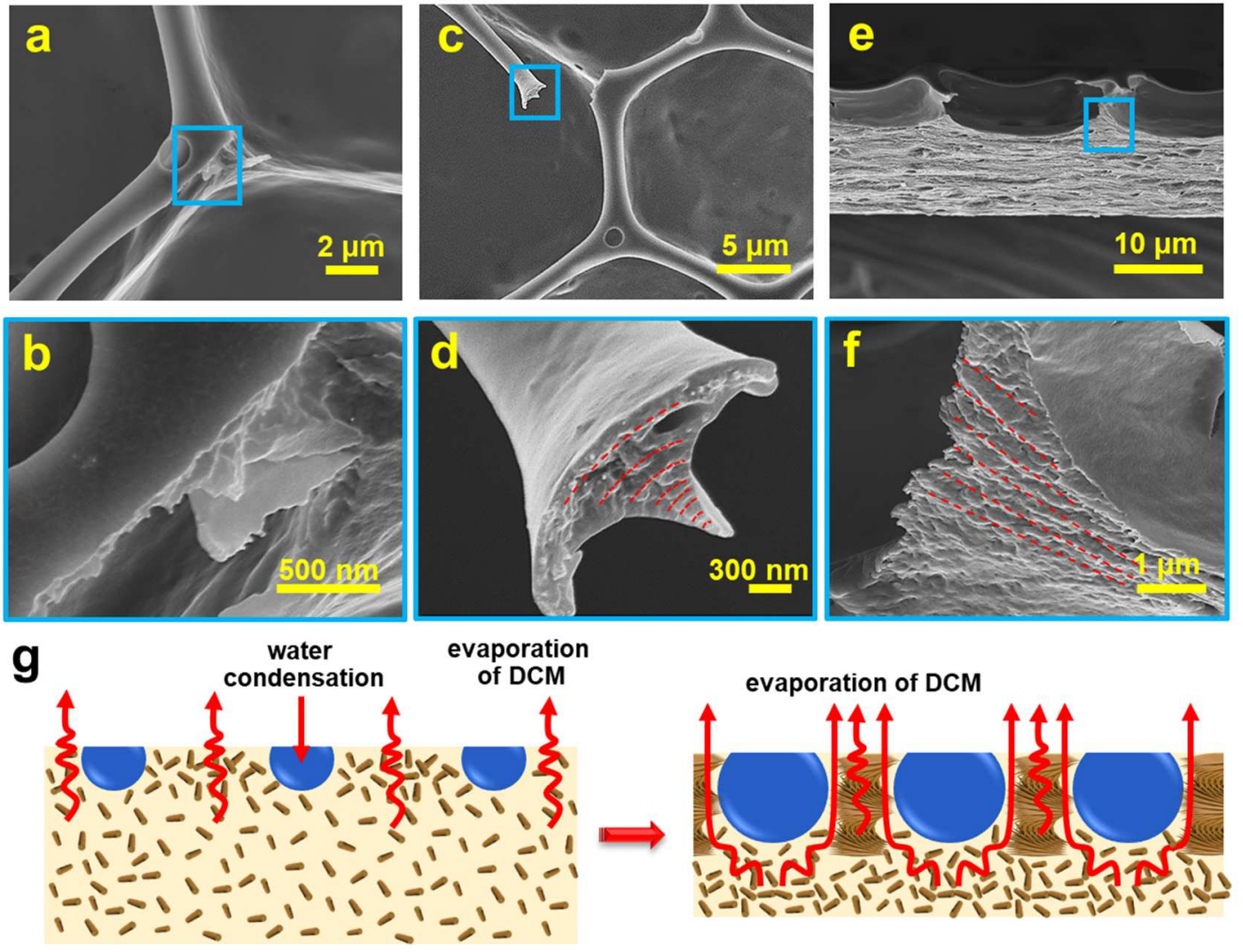

Figure 24. a) and c) Representative SEM image of the honeycomb film fabricated with CNWs-SU after removing part of walls of the pores. b) and d) SEM images of the magnified region as in (a) and (c), respectively. e) Representative SEM image of the cross section of the honeycomb film fabricated with CNWs-SU. f) SEM image of the magnified region as in (h). g) Schematic description for the formation of iridescent rim.

To understand the mechanism of the self-assembly of CNWs-SU into honeycomb films with iridescent rims, we detected the structures of the walls of pores. Figure 24a shows the top surface of honeycomb film after removing part of walls of the pores with a top view. The magnified SEM image clearly reveals the lamellar-like structure of the walls of pores (Figure $24 b$ ). From another SEM image of the damaged pores, we can see the cross section of the walls of pores present a ginkgo-leaf-like structure (Figure $24 \mathrm{c}, \mathrm{d}$ ). Figure $3 \mathrm{~d}$ shows the lamellar-like structure where CNWs-SU are organized into a chiral nematic structure with a 
periodic spacing of about $300 \mathrm{~nm}$. For the underside of the walls of pores, we can see the slandered lamellar structure (Figure 24e,f). This is mainly due to the width of the wall is getting narrower from bottom to top. With the growth of water droplets, CNWs-SU were squeezed and formed the slanted lamellar structure. We also proposed the mechanism for iridescent rim formation in honeycomb film during casting (Figure 3g). The CNWs-SU concentration was uniform in the initial aqueous suspension. However, as the water droplets formed on the surface of the suspension, the DCM will evaporate along with the areas where no water droplets formed. We predicted that two distinct regions would arise, one under the water droplets, where evaporation is restricted, and another outside the water droplets, where evaporation can occur. In the confined 3D walls, CNWs self-assembly into cholesteric architectures which resulted in the iridescent color of the rims. The arrangement presumably occurs upon the growth of the aqueous droplets during the BF process. The stage of selfassembly is very important in determining the resulting film's optical properties.
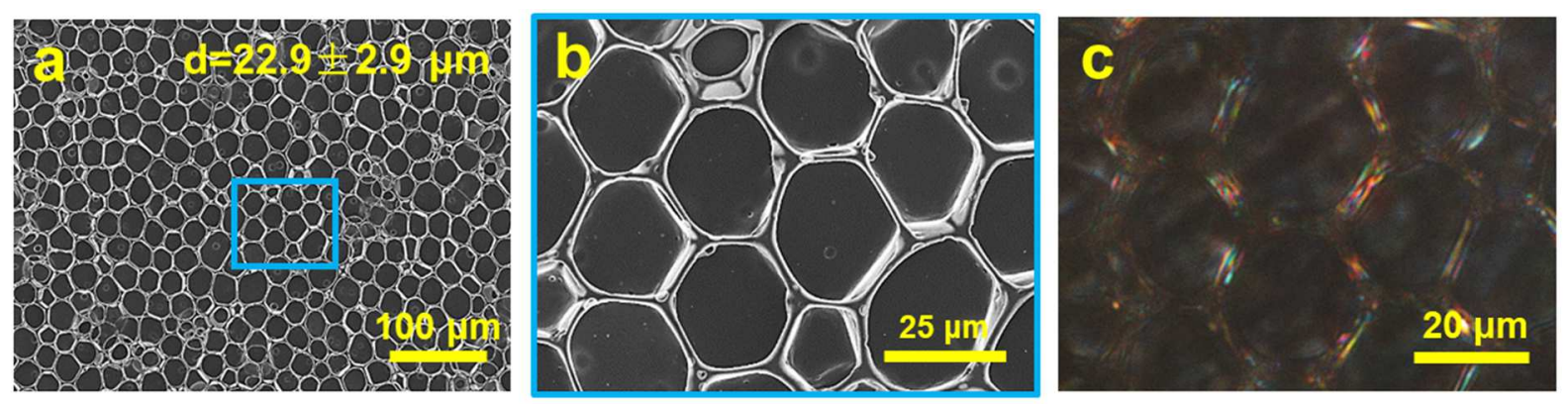

Figure 25. a) Representative SEM image of the top surface of the honeycomb film fabricated with CNWs-SU at the concentration of $7 \mathrm{mg} / \mathrm{mL}$. b) SEM image of the magnified region as in (a). c) PLM image of the top surface of the honeycomb film fabricated with CNWs-SU at the concentration of $7 \mathrm{mg} / \mathrm{mL}$.

The pore size and regularity of BF arrays are sensitive to the concentration of the solution. As a result, each CNWs solution with a different concentration yielded an entirely different morphology. However, when the concentration of CNWs was increased to $7 \mathrm{mg} / \mathrm{mL}$, the resulting films exhibited pores with irregular shapes (Figure 25a,b). On the other hand, the high concentration resulted in the poor self-assembly of CNWs in this kind of disorder geometry which shows in the not obvious iridescent color of the rim (Figure 25c). 

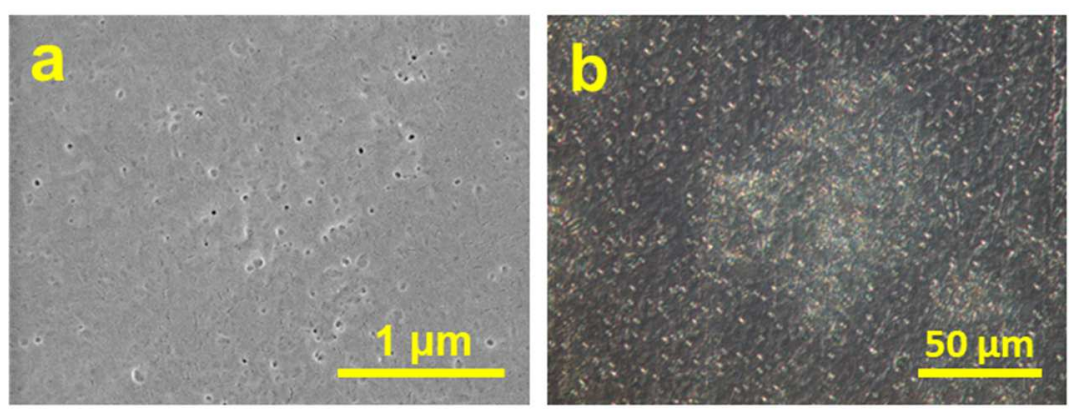

Figure 26. a) SEM image of the top surface of the honeycomb film fabricated with CNWsSU on the surface of Teflon with the concentration of $16 \mathrm{mg} / \mathrm{mL}(7 \mathrm{~mL})$. b) LSM image of the top surface of the honeycomb film fabricated with CNWs-SU on the surface of Teflon with the concentration of $3 \mathrm{mg} / \mathrm{mL}(5 \mathrm{~mL})$.

A malformed morphology was obtained when the concentration of CNWs was very high (Figure 26a). The increased viscosity will weaken the convection in the solution, which is not beneficial to the self-assembly of droplets, and the pores will become disordered when the concentration is very high. ${ }^{108}$ Honeycomb structures were not observed at the CNWs concentration at $3 \mathrm{mg} / \mathrm{mL}$ and the volume of $5 \mathrm{~mL}$ (Figure 26b). This result implies that an adequate amount of CNWs is required to form the honeycomb structures. This maybe stem from inefficient encapsulation and stabilization of the condensed water droplets levitation on the interface due to too low quantity of CNWs.
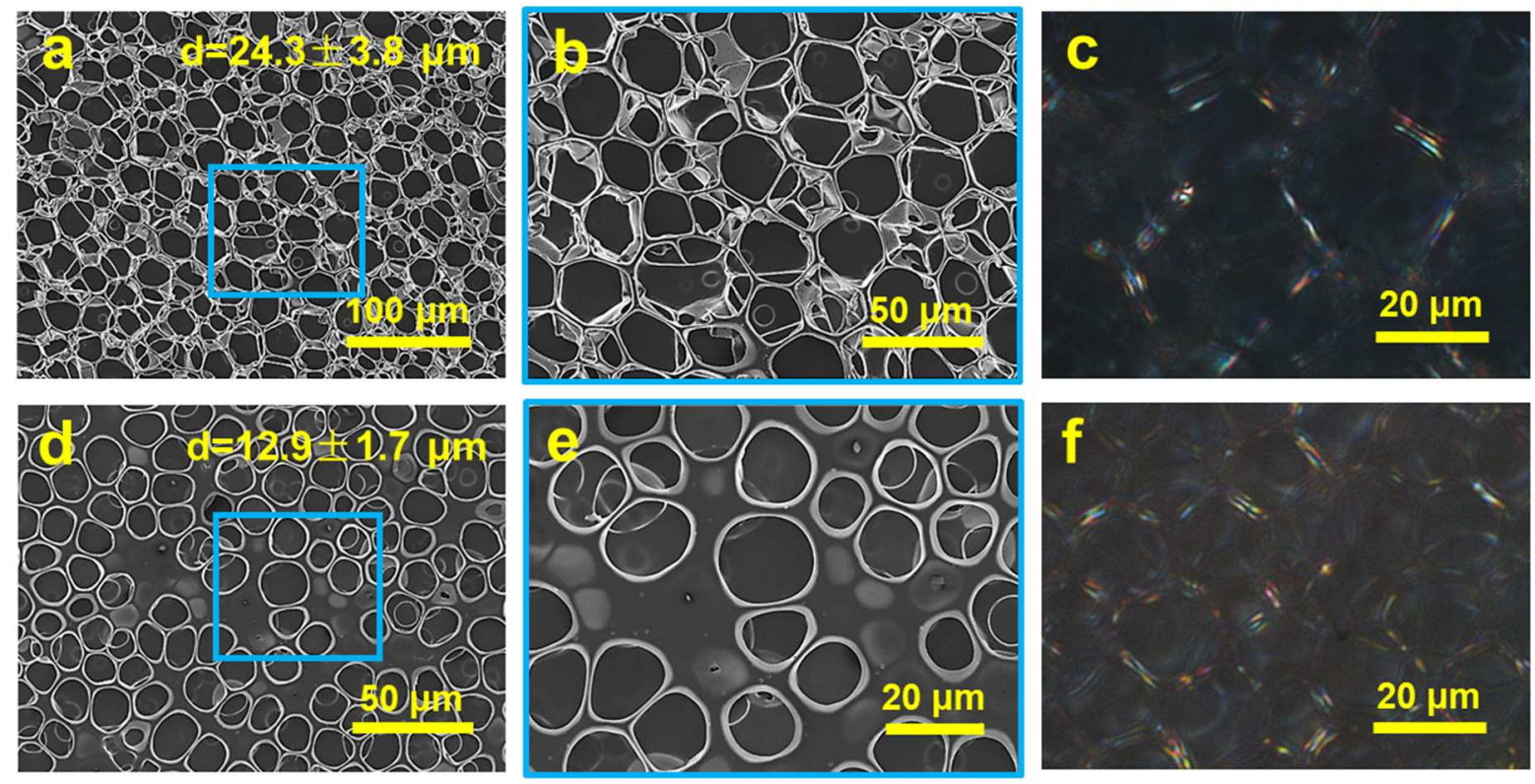
Figure 27. a) Representative SEM image of the top surface of the honeycomb film fabricated with CNWs-SU at $96 \%$ relative humidity. b) SEM image of the magnified region as in (a). c) PLM image of the top surface of the honeycomb film fabricated with CNWs-SU at $96 \%$ relative humidity. d) Representative SEM image of the top surface of the honeycomb film fabricated with CNWs-SU at $60 \%$ relative humidity. e) SEM image of the magnified region as in (d). f) PLM image of the top surface of the honeycomb film fabricated with CNWs-SU at $60 \%$ relative humidity.

As the humidity of the atmosphere increased from $75 \%$ to $96 \%$, the average pore size systematically increased from $17.0 \pm 2.8 \mu \mathrm{m}$ to $24.3 \pm 3.8 \mu \mathrm{m}$ (Figure $27 \mathrm{a}, \mathrm{b}$ ). The high humidity results in the coalescence of water droplets, yielding a polydisperse pore size distribution. The higher humidity not only forms larger water droplets but also slows the solvent evaporation, both of which lead to the growth of droplets and the formation of larger pores. ${ }^{109}$ When the relative humidity was $60 \%$, micropores uneven distributed on the top surface and even some areas did not form micropores (Figure $27 \mathrm{~d}, \mathrm{e}$ ). The humidity of the atmosphere influences the evaporation of the CNWs solvents, which further affect the self-assembly of CNWs in the confined 3D geometry (Figure 27c,f).

In conclusion, honeycomb films with iridescent rim were fabricated by self-assembly in breath figure templating of surface-modified cellulose nanowhiskers. 


\section{General conclusion and perspectives}

As mentioned all along this study, the self-assembly CNWs has attracted significant interest, resulting in much research effort, as this arrangement gives materials various functions. In this thesis, CNWs with surface-immobilized acyl groups CNWs-SU were synthesized through one-step esterification and the obtained CNWs well dissolved in nonpolar solvents. This study particularly focused on the self-assembly process of solely CNWs or coassembly with other nanoparticles as well as the self-assembly of CNWs-SU in confined geometry.

Firstly, in Publication 1, we show the formation of helical fibers with the lengths of micrometers by the evaporation-driven self-assembly on silicon wafers of functionalized CNWs with surface-attached acyl chains. The results show the significant effects of the wettability of substrate, dispersing solvents, the amount of 10-undecenoyl groups, the crystallinity, the length of CNWs and the length of acyl chains on the self-assembly process driven by the solvent evaporation. These parameters serve to adjust the self-assembly process and the final resulting helical structures.

Then, in Publication 2, we developed a facile one-step method to fabricate multiresponsive Janus films via the evaporation-driven self-assembly of surface-acylated CNWs bearing long alkane chains with or without GO. The heterogeneous nucleation of nanowhiskers on rough Teflon substrates favors the formation of thin films, whereas the homogeneous nucleation of residual nanowhiskers in bulk solutions promotes the growth of patchy microspheres that are composed of a large number of lamellar nanoplates. The incorporation of GO in the solution lowers the free-energy barrier of homogeneous nucleation and promotes the formation of more microparticles on the top surface. Janus films were obtained with diverse morphologies and wettabilities on both surfaces, while GO enhanced these differences on both surfaces. These microspheres precipitate on the top of the films and show thermo-reversible and reconstructable time-dependent donut structures in the dry-state. The structural difference between the top and bottom surfaces of GO-containing Janus films facilitates the solvent-responsive curling actuation in different solvents with fast reversible bending motions for many cycles.

Finally, in Publication 3, a porous CNWs-SU film with well-ordered porous structures was prepared by using the breath figure method. The rim of the obtained honeycomb film displayed iridescent color due to the confined 3D geometry drastically affects the self- 
assembly of CNWs. The rims, composed of stacked and overlapped CNWs, are highly arranged along the boundary of micropores. The arrangement presumably occurs upon the growth of the aqueous droplets during the breath-figure process. Additionally, some critical influencing factors, such as the concentration of the CNWs solution and the relative humidity of the atmosphere were also investigated.

In conclusion, the various studies described in this thesis highlighted the unique selfassembly behavior of the surface-acylated CNWs. Remarkably, microscale fibers with explicit helical structures were fabricated in Publication 1. These self-assembled helical structures can endow materials with many excellent properties for potentially broad applications. Moreover, the proposed self-assembly approach would open up a promising avenue for growing and patterning nanostructures using surface-functionalized nanocellulose. In addition, we found that the thermo-reversible self-assembly from CNWs is possible in dry state and the self-assembly can be tuned by adding GO. These findings not only extend our understanding of the self-assembly of anisotropic nanorods dominated by one mechanism toward those with combined mechanisms, e.g. the interfacial activity on (liquid-substrate) interface and the bulk structure formation in solutions, but also provide inspirations in designing and fabricating other functional Janus materials. Moreover, honeycomb films with iridescent rim were fabricated with CNWs via breath figure method. This simple technique unlocks new possibilities in designing the visual appearance of such iridescent films. We expect these porous films with structural color may lead to a broad application of sophisticated optical materials. 


\section{Reference}

1. Brinchi, L.; Cotana, F.; Fortunati, E.; Kenny, J. M., Production of nanocrystalline cellulose from lignocellulosic biomass: Technology and applications. Carbohydrate Polymers 2013, 94 (1), 154-169.

2. Thakur, V. K.; Thakur, M. K., Processing and characterization of natural cellulose fibers/thermoset polymer composites. Carbohydrate Polymers 2014, 109, 102-117.

3. Gardner, K. H.; Blackwell, J., The structure of native cellulose. Biopolymers 1974, 13 (10), 1975-2001.

4. Sjostrom, E., Wood chemistry: fundamentals and applications. Academic Press, Inc.: New York, 1981.

5. Klemm, D.; Heublein, B.; Fink, H.-P.; Bohn, A., Cellulose: fascinating biopolymer and sustainable raw material. Angewandte Chemie International Edition 2005, 44 (22), 33583393.

6. Moon, R. J.; Martini, A.; Nairn, J.; Simonsen, J.; Youngblood, J., Cellulose nanomaterials review: structure, properties and nanocomposites. Chemical Society Reviews 2011, 40 (7), 3941-3994.

7. Marcos, M.; Nadia, E. K.; Alain, D., Cellulose nanocrystals and related nanocomposites: review of some properties and challenges. Journal of Polymer Science Part B: Polymer Physics 2014, 52 (12), 791-806.

8. Trache, D.; Hussin, M. H.; Haafiz, M. K. M.; Thakur, V. K., Recent progress in cellulose nanocrystals: sources and production. Nanoscale 2017, 9 (5), 1763-1786.

9. Lin, N.; Dufresne, A., Nanocellulose in biomedicine: current status and future prospect. European Polymer Journal 2014, 59, 302-325.

10. Thomas, B.; Raj, M. C.; B, A. K.; H, R. M.; Joy, J.; Moores, A.; Drisko, G. L.; Sanchez, C., Nanocellulose, a versatile green platform: from biosources to materials and their applications. Chemical Reviews 2018, 118 (24), 11575-11625.

11. Habibi, Y., Key advances in the chemical modification of nanocelluloses. Chemical Society Reviews 2014, 43 (5), 1519-1542.

12. Bondeson, D.; Mathew, A.; Oksman, K., Optimization of the isolation of nanocrystals from microcrystalline celluloseby acid hydrolysis. Cellulose 2006, 13 (2), 171.

13. Sun, B.; Zhang, M.; Hou, Q.; Liu, R.; Wu, T.; Si, C., Further characterization of cellulose nanocrystal (CNC) preparation from sulfuric acid hydrolysis of cotton fibers. Cellulose 2016, 23 (1), 439-450. 
14. Wang, N.; Ding, E.; Cheng, R., Preparation and liquid crystalline properties of spherical cellulose nanocrystals. Langmuir 2008, 24 (1), 5-8.

15. Yu, H.; Qin, Z.; Liang, B.; Liu, N.; Zhou, Z.; Chen, L., Facile extraction of thermally stable cellulose nanocrystals with a high yield of $93 \%$ through hydrochloric acid hydrolysis under hydrothermal conditions. Journal of Materials Chemistry A 2013, 1 (12), 3938-3944.

16. Habibi, Y.; Lucia, L. A.; Rojas, O. J., Cellulose nanocrystals: chemistry, self-assembly, and applications. Chemical Reviews 2010, 110 (6), 3479-3500.

17. Wang, Y.; Wang, X.; Xie, Y.; Zhang, K., Functional nanomaterials through esterification of cellulose: a review of chemistry and application. Cellulose 2018, 25 (7), 3703-3731.

18. George, J.; Sabapathi, S. N., Cellulose nanocrystals: synthesis, functional properties, and applications. Nanotechnol Sci Appl 2015, 8, 45-54.

19. Habibi, Y.; Chanzy, H.; Vignon, M. R., TEMPO-mediated surface oxidation of cellulose whiskers. Cellulose 2006, 13 (6), 679-687.

20. Salajková, M.; Berglund, L. A.; Zhou, Q., Hydrophobic cellulose nanocrystals modified with quaternary ammonium salts. Journal of Materials Chemistry 2012, 22 (37), 19798-19805.

21. Uhlig, M.; Fall, A.; Wellert, S.; Lehmann, M.; Prévost, S.; Wågberg, L.; von Klitzing, R.; Nyström, G., Two-dimensional aggregation and semidilute ordering in cellulose nanocrystals. Langmuir 2016, 32 (2), 442-450.

22. Fraschini, C.; Chauve, G.; Bouchard, J., TEMPO-mediated surface oxidation of cellulose nanocrystals (CNCs). Cellulose 2017, 24 (7), 2775-2790.

23. Bragd, P.; Van Bekkum, H.; Besemer, A., TEMPO-mediated oxidation of polysaccharides: survey of methods and applications. Topics in Catalysis 2004, 27, 49-66.

24. De Nooy, A.; Besemer, A.; Van Bekkum, H., Highly selective TEMPO mediated oxidation of primary alcohol groups in polysaccharides. Recueil des Travaux Chimiques des Pays-Bas 1994, 113 (3), 165-166.

25. De Nooy, A. E.; Besemer, A. C.; van Bekkum, H., Highly selective nitroxyl radicalmediated oxidation of primary alcohol groups in water-soluble glucans. Carbohydrate research 1995, 269 (1), 89-98.

26. De Nooy, A.; Besemer, A. C.; Van Bekkum, H.; Van Dijk, J.; Smit, J., TEMPOmediated oxidation of pullulan and influence of ionic strength and linear charge density on the dimensions of the obtained polyelectrolyte chains. Macromolecules 1996, 29 (20), 6541-6547. 
27. Chang, P. S.; Robyt, J. F., Oxidation of primary alcohol groups of naturally occurring polysaccharides with 2,2,6,6-tetramethyl-1-piperidine oxoammonium ion. Journal of Carbohydrate Chemistry 1996, 15 (7), 819-830.

28. Montanari, S.; Roumani, M.; Heux, L.; Vignon, M. R., Topochemistry of carboxylated cellulose nanocrystals resulting from TEMPO-mediated oxidation. Macromolecules 2005, 38 (5), 1665-1671.

29. Li, B.; Xu, W.; Kronlund, D.; Määttänen, A.; Liu, J.; Smått, J.-H.; Peltonen, J.; Willför, $\mathrm{S}$; $\mathrm{Mu}, \mathrm{X} . ; \mathrm{Xu}, \mathrm{C}$., Cellulose nanocrystals prepared via formic acid hydrolysis followed by TEMPO-mediated oxidation. Carbohydrate Polymers 2015, 133, 605-612.

30. Abitbol, T.; Kloser, E.; Gray, D. G., Estimation of the surface sulfur content of cellulose nanocrystals prepared by sulfuric acid hydrolysis. Cellulose 2013, 20 (2), 785-794.

31. Gu, J.; Catchmark, J. M.; Kaiser, E. Q.; Archibald, D. D., Quantification of cellulose nanowhiskers sulfate esterification levels. Carbohydrate Polymers 2013, 92 (2), 1809-1816.

32. Araki, J.; Wada, M.; Kuga, S.; Okano, T., Influence of surface charge on viscosity behavior of cellulose microcrystal suspension. Journal of Wood Science 1999, 45 (3), 258-261. 33. Araki, J.; Wada, M.; Kuga, S.; Okano, T., Birefringent glassy phase of a cellulose microcrystal suspension. Langmuir 2000, 16 (6), 2413-2415.

34. Lin, N.; Dufresne, A., Surface chemistry, morphological analysis and properties of cellulose nanocrystals with gradiented sulfation degrees. Nanoscale 2014, 6 (10), 5384-5393.

35. Roman, M.; Winter, W. T., Effect of sulfate groups from sulfuric acid hydrolysis on the thermal degradation behavior of bacterial cellulose. Biomacromolecules 2004, 5 (5), 1671 1677.

36. Li, L.; Ma, W.; Higaki, Y.; Kamitani, K.; Takahara, A., Organic-inorganic hybrid thin films fabricated by layer-by-layer assembly of the phosphorylated cellulose nanocrystal and imogolite nanotubes. Langmuir 2018, 34 (44), 13361-13367.

37. Baek, J.; Wahid-Pedro, F.; Kim, K.; Kim, K.; Tam, K. C., Phosphorylated$\mathrm{CNC} /$ modified-chitosan nanocomplexes for the stabilization of Pickering emulsions. Carbohydrate Polymers 2019, 206, 520-527.

38. Kim, J.; Montero, G.; Habibi, Y.; Hinestroza, J. P.; Genzer, J.; Argyropoulos, D. S.; Rojas, O. J., Dispersion of cellulose crystallites by nonionic surfactants in a hydrophobic polymer matrix. Polymer Engineering \& Science 2009, 49 (10), 2054-2061. 
39. Khoshkava, V.; Kamal, M. R., Effect of drying conditions on cellulose nanocrystal (CNC) agglomerate porosity and dispersibility in polymer nanocomposites. Powder Technology 2014, 261, 288-298.

40. Mormann, W.; Al-Higari, M., Acylation of starch with vinyl acetate in water. Starch-Stärke 2004, 56 (3-4), 118-121.

41. Dicke, R., A straight way to regioselectively functionalized polysaccharide esters. Cellulose 2004, 11 (2), 255-263.

42. Heinze, T.; Dicke, R.; Koschella, A.; Kull, A. H.; Klohr, E. A.; Koch, W., Effective preparation of cellulose derivatives in a new simple cellulose solvent. Macromolecular Chemistry and Physics 2000, 201 (6), 627-631.

43. Xie, J.; Hsieh, Y. L., Enzyme-catalyzed transesterification of vinyl esters on cellulose solids. Journal of Polymer Science Part A: Polymer Chemistry 2001, 39 (11), 1931-1939.

44. Yang, K.; Wang, Y. J., Lipase-catalyzed cellulose acetylation in aqueous and organic media. Biotechnology progress 2003, 19 (6), 1664-1671.

45. Çetin, N. S.; Tingaut, P.; Özmen, N.; Henry, N.; Harper, D.; Dadmun, M.; Sèbe, G., Acetylation of cellulose nanowhiskers with vinyl acetate under moderate conditions. Macromolecular Bioscience 2009, 9 (10), 997-1003.

46. Yuan, H.; Nishiyama, Y.; Wada, M.; Kuga, S., Surface acylation of cellulose whiskers by drying aqueous emulsion. Biomacromolecules 2006, 7 (3), 696-700.

47. Braun, B.; Dorgan, J. R., Single-step method for the isolation and surface functionalization of cellulosic nanowhiskers. Biomacromolecules 2009, 10 (2), 334-341.

48. Junior de Menezes, A.; Siqueira, G.; Curvelo, A. A. S.; Dufresne, A., Extrusion and characterization of functionalized cellulose whiskers reinforced polyethylene nanocomposites. Polymer 2009, 50 (19), 4552-4563.

49. Liu, H.; Pang, B.; Garces, R.; Dervisoglu, R.; Chen, L.; Andreas, L.; Zhang, K., Helical fibers via evaporation-driven self-assembly of surface-acylated cellulose nanowhiskers. Angewandte Chemie International Edition 2018, 57 (50), 16323-16328.

50. Hasani, M.; Cranston, E. D.; Westman, G.; Gray, D. G., Cationic surface functionalization of cellulose nanocrystals. Soft Matter 2008, 4 (11), 2238-2244.

51. Zaman, M.; Xiao, H.; Chibante, F.; Ni, Y., Synthesis and characterization of cationically modified nanocrystalline cellulose. Carbohydrate Polymers 2012, 89 (1), 163 170. 
52. Goussé, C.; Chanzy, H.; Excoffier, G.; Soubeyrand, L.; Fleury, E., Stable suspensions of partially silylated cellulose whiskers dispersed in organic solvents. Polymer 2002, 43 (9), 2645-2651.

53. Pei, A.; Zhou, Q.; Berglund, L. A., Functionalized cellulose nanocrystals as biobased nucleation agents in poly(l-lactide) (PLLA) - Crystallization and mechanical property effects. Composites Science and Technology 2010, 70 (5), 815-821.

54. Saini, S.; Belgacem, M. N.; Salon, M.-C. B.; Bras, J., Non leaching biomimetic antimicrobial surfaces via surface functionalisation of cellulose nanofibers with aminosilane. Cellulose 2016, 23 (1), 795-810.

55. Khanjanzadeh, H.; Behrooz, R.; Bahramifar, N.; Gindl-Altmutter, W.; Bacher, M.; Edler, M.; Griesser, T., Surface chemical functionalization of cellulose nanocrystals by 3aminopropyltriethoxysilane. International Journal of Biological Macromolecules 2018, 106, 1288-1296.

56. Marchessault, R.; Morehead, F.; Walter, N., Liquid crystal systems from fibrillar polysaccharides. Nature 1959, 184 (4686), 632-633.

57. Revol, J. F.; Bradford, H.; Giasson, J.; Marchessault, R. H.; Gray, D. G., Helicoidal self-ordering of cellulose microfibrils in aqueous suspension. International Journal of Biological Macromolecules 1992, 14 (3), 170-172.

58. Lagerwall, J. P.; Scalia, G., A new era for liquid crystal research: Applications of liquid crystals in soft matter nano-, bio-and microtechnology. Current Applied Physics 2012, $12(6), 1387-1412$.

59. Lagerwall, J. P. F.; Schütz, C.; Salajkova, M.; Noh, J.; Hyun Park, J.; Scalia, G.; Bergström, L., Cellulose nanocrystal-based materials: from liquid crystal self-assembly and glass formation to multifunctional thin films. Npg Asia Materials 2014, 6, e80.

60. Eichhorn, S. J., Cellulose nanowhiskers: promising materials for advanced applications. Soft Matter 2011, 7 (2), 303-315.

61. Usov, I.; Nyström, G.; Adamcik, J.; Handschin, S.; Schütz, C.; Fall, A.; Bergström, L.; Mezzenga, R., Understanding nanocellulose chirality and structure-properties relationship at the single fibril level. Nature communications 2015, 6 (1), 1-11.

62. Conley, K.; Godbout, L.; Whitehead, M. A.; van de Ven, T. G. M., Origin of the twist of cellulosic materials. Carbohydrate Polymers 2016, 135, 285-299. 
63. Parker, R. M.; Guidetti, G.; Williams, C. A.; Zhao, T.; Narkevicius, A.; Vignolini, S.; Frka-Petesic, B., The self-assembly of cellulose nanocrystals: hierarchical design of visual appearanceh. Advanced Materials 2018, 30 (19), 1704477.

64. Dong, X. M.; Kimura, T.; Revol, J.-F.; Gray, D. G., Effects of ionic strength on the isotropic-chiral nematic phase transition of suspensions of cellulose crystallites. Langmuir 1996, 12 (8), 2076-2082.

65. Gray, D. G.; Mu, X., Chiral nematic structure of cellulose nanocrystal suspensions and films; polarized light and atomic force microscopy. Materials 2015, 8 (11), 7873-7888.

66. Revol, J.-F.; Godbout, L.; Dong, X.-M.; Gray, D. G.; Chanzy, H.; Maret, G., Chiral nematic suspensions of cellulose crystallites; phase separation and magnetic field orientation. Liquid Crystals 1994, 16 (1), 127-134.

67. de Souza Lima, M. M.; Borsali, R., Rodlike cellulose microcrystals: structure, properties, and applications. Macromolecular rapid communications 2004, 25 (7), 771-787.

68. Majoinen, J.; Kontturi, E.; Ikkala, O.; Gray, D. G., SEM imaging of chiral nematic films cast from cellulose nanocrystal suspensions. Cellulose 2012, 19 (5), 1599-1605.

69. $\mathrm{Mu}, \mathrm{X}$.; Gray, D. G., Formation of chiral nematic films from cellulose nanocrystal suspensions is a two-stage process. Langmuir 2014, 30 (31), 9256-9260.

70. Beck-Candanedo, S.; Roman, M.; Gray, D. G., Effect of reaction conditions on the properties and behavior of wood cellulose nanocrystal suspensions. Biomacromolecules 2005, $6(2), 1048-1054$.

71. Pan, J.; Hamad, W.; Straus, S. K., Parameters affecting the chiral nematic phase of nanocrystalline cellulose films. Macromolecules 2010, 43 (8), 3851-3858.

72. Beck, S.; Bouchard, J.; Chauve, G.; Berry, R., Controlled production of patterns in iridescent solid films of cellulose nanocrystals. Cellulose 2013, 20 (3), 1401-1411.

73. Dong, X. M.; Revol, J.-F.; Gray, D. G., Effect of microcrystallite preparation conditions on the formation of colloid crystals of cellulose. Cellulose 1998, 5 (1), 19-32.

74. Beck, S.; Bouchard, J.; Berry, R., Controlling the reflection wavelength of iridescent solid films of nanocrystalline cellulose. Biomacromolecules 2011, 12 (1), 167-172.

75. Dong, X. M.; Gray, D. G., Effect of counterions on ordered phase formation in suspensions of charged rodlike cellulose crystallites. Langmuir 1997, 13 (8), 2404-2409.

76. Ličen, M.; Majaron, B.; Noh, J.; Schütz, C.; Bergström, L.; Lagerwall, J.; DrevenšekOlenik, I., Correlation between structural properties and iridescent colors of cellulose nanocrystalline films. Cellulose 2016, 23 (6), 3601-3609. 
77. Ebeling, T.; Paillet, M.; Borsali, R.; Diat, O.; Dufresne, A.; Cavaillé, J. Y.; Chanzy, H., Shear-induced orientation phenomena in suspensions of cellulose microcrystals, revealed by small angle X-ray scattering. Langmuir 1999, 15 (19), 6123-6126.

78. Chowdhury, R. A.; Peng, S. X.; Youngblood, J., Improved order parameter (alignment) determination in cellulose nanocrystal (CNC) films by a simple optical birefringence method. Cellulose 2017, 24 (5), 1957-1970.

79. Yoshiharu, N.; Shigenori, K.; Masahisa, W.; Takeshi, O., Cellulose microcrystal film of high uniaxial orientation. Macromolecules 1997, 30 (20), 6395-6397.

80. Tatsumi, M.; Teramoto, Y.; Nishio, Y., Different orientation patterns of cellulose nanocrystal films prepared from aqueous suspensions by shearing under evaporation. Cellulose 2015, 22 (5), 2983-2992.

81. Zong, Y.; Yue, Z.; Higgins, M. J., Nanocrystalline cellulose for anisotropic magnetoelectric composites. Macromolecular Materials and Engineering 2018, 303 (7), 1800099.

82. Pullawan, T.; Wilkinson, A. N.; Eichhorn, S. J., Orientation and deformation of wetstretched all-cellulose nanocomposites. Journal of Materials Science 2013, 48 (22), 78477855.

83. Osorio-Madrazo, A.; Eder, M.; Rueggeberg, M.; Pandey, J. K.; Harrington, M. J.; Nishiyama, Y.; Putaux, J.-L.; Rochas, C.; Burgert, I., Reorientation of cellulose nanowhiskers in agarose hydrogels under tensile loading. Biomacromolecules 2012, 13 (3), 850-856.

84. Kose, O.; Tran, A.; Lewis, L.; Hamad, W. Y.; MacLachlan, M. J., Unwinding a spiral of cellulose nanocrystals for stimuli-responsive stretchable optics. Nature Communications 2019, $10(1), 510$.

85. Kose, O.; Boott, C. E.; Hamad, W. Y.; MacLachlan, M. J., Stimuli-responsive anisotropic materials based on unidirectional organization of cellulose nanocrystals in an elastomer. Macromolecules 2019, 52 (14), 5317-5324.

86. Huang, H.; Wang, X.; Yu, J.; Chen, Y.; Ji, H.; Zhang, Y.; Rehfeldt, F.; Wang, Y.; Zhang, K., Liquid-behaviors-assisted fabrication of multidimensional birefringent materials from dynamic hybrid hydrogels. ACS Nano 2019, 13 (4), 3867-3874.

87. Qu, D.; Chu, G.; Martin, P.; Vasilyev, G.; Vilensky, R.; Zussman, E., Modulating the structural orientation of nanocellulose composites through mechano-stimuli. ACS Applied Materials \& Interfaces 2019, 11 (43), 40443-40450. 
88. Giese, M.; Khan, M. K.; Hamad, W. Y.; MacLachlan, M. J., Imprinting of photonic patterns with thermosetting amino-formaldehyde-cellulose composites. ACS Macro Letters 2013, 2 (9), 818-821.

89. Kamita, G.; Frka-Petesic, B.; Allard, A.; Dargaud, M.; King, K.; Dumanli, A. G.; Vignolini, S., Biocompatible and sustainable optical strain sensors for large-area applications. Advanced Optical Materials 2016, 4 (12), 1950-1954.

90. Bordel, D.; Putaux, J.-L.; Heux, L., Orientation of native cellulose in an electric field. Langmuir 2006, 22 (11), 4899-4901.

91. Habibi, Y.; Heim, T.; Douillard, R., AC electric field-assisted assembly and alignment of cellulose nanocrystals. Journal of Polymer Science Part B: Polymer Physics 2008, 46 (14), $1430-1436$.

92. Csoka, L.; Hoeger, I. C.; Peralta, P.; Peszlen, I.; Rojas, O. J., Dielectrophoresis of cellulose nanocrystals and alignment in ultrathin films by electric field-assisted shear assembly. Journal of Colloid and Interface Science 2011, 363 (1), 206-212.

93. Frka-Petesic, B.; Radavidson, H.; Jean, B.; Heux, L., Dynamically controlled iridescence of cholesteric cellulose nanocrystal suspensions using electric fields. Advanced Materials 2017, 29 (11), 1606208.

94. Sugiyama, J.; Chanzy, H.; Maret, G., Orientation of cellulose microcrystals by strong magnetic fields. Macromolecules 1992, 25 (16), 4232-4234.

95. De France, K. J.; Yager, K. G.; Hoare, T.; Cranston, E. D., Cooperative ordering and kinetics of cellulose nanocrystal alignment in a magnetic field. Langmuir 2016, 32 (30), 7564 7571.

96. Frka-Petesic, B.; Guidetti, G.; Kamita, G.; Vignolini, S., Controlling the photonic properties of cholesteric cellulose nanocrystal films with magnets. Advanced Materials 2017, 29 (32), 1701469.

97. Shopsowitz, K. E.; Qi, H.; Hamad, W. Y.; MacLachlan, M. J., Free-standing mesoporous silica films with tunable chiral nematic structures. Nature 2010, 468, 422.

98. Qi, H.; Shopsowitz, K. E.; Hamad, W. Y.; MacLachlan, M. J., Chiral nematic assemblies of silver nanoparticles in mesoporous silica thin films. Journal of the American Chemical Society 2011, 133 (11), 3728-3731.

99. Kelly, J. A.; Shopsowitz, K. E.; Ahn, J. M.; Hamad, W. Y.; MacLachlan, M. J., Chiral nematic stained glass: controlling the optical properties of nanocrystalline cellulose-templated materials. Langmuir 2012, 28 (50), 17256-17262. 
100. Shopsowitz, K. E.; Stahl, A.; Hamad, W. Y.; MacLachlan, M. J., Hard templating of nanocrystalline titanium dioxide with chiral nematic ordering. Angewandte Chemie International Edition 2012, 51 (28), 6886-6890.

101. Shopsowitz, K. E.; Hamad, W. Y.; MacLachlan, M. J., Chiral nematic mesoporous carbon derived from nanocrystalline cellulose. Angewandte Chemie International Edition 2011, 50 (46), 10991-10995.

102. Querejeta-Fernández, A.; Chauve, G.; Methot, M.; Bouchard, J.; Kumacheva, E., Chiral plasmonic films formed by gold nanorods and cellulose nanocrystals. Journal of the American Chemical Society 2014, 136 (12), 4788-4793.

103. Cheng, Z.; Ma, Y.; Yang, L.; Cheng, F.; Huang, Z.; Natan, A.; Li, H.; Chen, Y.; Cao, D.; Huang, Z.; Wang, Y.; Liu, Y.; Yang, R.; Zhu, H., Plasmonic-enhanced cholesteric films: coassembling anisotropic gold nanorods with cellulose nanocrystals. Advanced Optical Materials 2019, 7, 1801816.

104. Chu, G.; Wang, X.; Chen, T.; Gao, J.; Gai, F.; Wang, Y.; Xu, Y., Optically tunable chiral plasmonic guest-host cellulose films weaved with long-range ordered silver nanowires. ACS Applied Materials \& Interfaces 2015, 7 (22), 11863-11870.

105. Thérien-Aubin, H.; Lukach, A.; Pitch, N.; Kumacheva, E., Coassembly of nanorods and nanospheres in suspensions and in stratified films. Angewandte Chemie International Edition 2015, 54 (19), 5618-5622.

106. Thérien-Aubin, H.; Lukach, A.; Pitch, N.; Kumacheva, E., Structure and properties of composite films formed by cellulose nanocrystals and charged latex nanoparticles. Nanoscale 2015, 7 (15), 6612-6618.

107. Chen, Q.; Liu, P.; Sheng, C.; Zhou, L.; Duan, Y.; Zhang, J., Tunable self-assembly structure of graphene oxide/cellulose nanocrystal hybrid films fabricated by vacuum filtration technique. RSC Advances 2014, 4 (74), 39301-39304.

108. Zhao, B.; Zhang, J.; Wang, X.; Li, C., Water-assisted fabrication of honeycomb structure porous film from poly(l-lactide). Journal of Materials Chemistry 2006, 16 (5), 509513.

109. Wang, C.; Mao, Y.; Wang, D.; Qu, Q.; Yang, G.; Hu, X., Fabrication of highly ordered microporous thin films by PS-b-PAA self-assembly and investigation of their tunable surface properties. Journal of Materials Chemistry 2008, 18 (6), 683-690. 


\section{Appendix}

Author's peer-reviewed publications 


\section{Publication 1}

Helical fibers via evaporation-driven self-assembly of surface-acylated cellulose nanowhiskers

Huan Liu, Bo Pang, Renata Garces, Riza Dervişoğlu, Longquan Chen, Loren Andreas, and Kai Zhang*

The article was published in Angewandte Chemie International Edition 57 (50), P 16323 16328 in December 2018. The supplementary materials are attached to the main part. The full article can be found online at:

https://onlinelibrary.wiley.com/doi/abs/10.1002/anie.201808250

DOI: $\underline{10.1002 / \text { anie. } 201808250}$ 


\title{
Helical Fibers via Evaporation-Driven Self-Assembly of Surface- Acylated Cellulose Nanowhiskers
}

\author{
Huan Liu, Bo Pang, Renata Garces, Riza Dervisoglu, Longquan Chen, Loren Andreas, and \\ Kai Zhang*
}

\begin{abstract}
Many natural materials have helical or twisting shapes. Herein, we show the formation of helical fibers with the lengths of micrometers by the evaporation-driven self-assembly on silicon wafers of functionalized cellulose nanowhiskers (CNWs) with surface-attached acyl chains. The self-assembly process and the final helical structures were affected by parameters including the wettability of substrates, dispersing solvents, the amount of 10-undecenoyl groups, the crystallinity, the dimension of CNWs, and the length of acyl chains. In particular, surface-acylated CNWs with a certain amount of 10undecenoyl groups (ca. $3.52 \mathrm{mmolg}^{-1}$ ), an appropriate crystallinity (ca. $40 \%$ ), a length of about $135 \mathrm{~nm}$, and a diameter of around $4 \mathrm{~nm}$, preferentially self-assembled into explicit lefthanded helical fibers from their THF suspensions on wafers. Thus, we showed novel particular self-assembly behaviors of surface-acylated CNWs, and we expanded the materials spectrum for the construction of helical structures.
\end{abstract}

Helical architectures are ubiquitous in nature. A wellknown example is climbing tendrils, which provide a parasitic alternative to help some plants attach to trees or other objects. ${ }^{[1]}$ Also the animal kingdom often features the presence of helical or spiral macro-morphologies, such as the horns of some animals and sea shells. ${ }^{[2]}$ In addition to these helical architectures at macroscopic scale, a few native macromolecules have helical architectures. The double helices formed by DNA (pitch of $3.4 \mathrm{~nm})^{[3]}$ and the $\alpha$-helical motifs in proteins (pitch of $0.54 \mathrm{~nm}$ ),${ }^{[4]}$ for example, generate stable structures with critical roles in the three-dimensional

[*] H. Liu, B. Pang, Prof. Dr. K. Zhang

Wood Technology and Wood Chemistry

Georg-August-University of Göttingen

37077 Göttingen (Germany)

E-mail: kai.zhang@uni-goettingen.de

Dr. R. Garces

Third Institute of Physics-Biophysics, Georg-August-University of

Göttingen

37077 Göttingen (Germany)

Dr. R. Dervisoglu, Dr. L. Andreas

NMR-based Structural Biology, Max-Planck-Institute for Biophysical Chemistry

37077 Göttingen (Germany)

Prof. Dr. L. Chen

School of Physics, University of Electronic Science and Technology of China

610054 Chengdu (China)

(iD)

Supporting information and the ORCID identification number(s) for the author(s) of this article can be found under:

https://doi.org/10.1002/anie.201808250. architectures in biology. ${ }^{[5]}$ Another archetypal example is collagen, a right-handed superhelical structure of $0.95 \mathrm{~nm}$ pitch, formed through self-assembly of three left-handed amino-acid based chains during the biosynthesis. ${ }^{[6]}$

Inspired by the unique features of fascinating biological superstructures, the creation of even more complex architectures has recently become possible by applying the concept of self-assembly. ${ }^{[7]}$ Among various self-assembled nanostructures, helical architectures have attracted special attention in view of their chiroptical properties, supramolecular chiral chemistry and supramolecular electronics. ${ }^{[8]}$ Amyloid fibers, a natural chiral protein-based system, formed through the self-assembly of $\beta$-sheet aggregates into twisted or helical ribbons. ${ }^{[9]}$ These fibers have found various applications in nanotechnology and biomaterials as templates or building blocks due to their unique physical and mechanical properties. ${ }^{[10]}$ A variety of aesthetically appealing helical structures have been created through the assembly of synthetic polymers or inorganic substance. These spiral nano-/micromaterials have found diverse applications in the area of dynamic functional materials through the transformation of high-order supramolecular architectures using external stimuli, such as light, solvents or ions. ${ }^{[1]]}$ However, helical microfibers constructed by self-assembled polysaccharides-based nanomaterials have not been reported.

Herein, we show for the first time that cellulose nanowhiskers (CNWs) with surface-attached acyl chains form helical fibers with the lengths of micrometers induced by solvent evaporation on silicon wafers. Various surface-acylated CNWs containing diverse acyl groups with the diameters of a few $\mathrm{nm}$ and the length of around $100 \mathrm{~nm}$ were prepared and used for the formation of helical fibers under varied conditions. CNWs have attracted much attention owing to their outstanding properties, such as high surface to volume ratio, high mechanical performance, small thermal expansion coefficient, renewability and sustainability. ${ }^{[12]}$ As CNWs are rigid rod-like particles generally with the length of $100-300 \mathrm{~nm}$ and the diameter of 3-10 nm, they have a strong tendency to align along a vector director. ${ }^{[13]} \mathrm{CNWs}$ with a threshold concentration in aqueous suspensions form cholesteric structures that can be retained upon solvent evaporation to form iridescent films. ${ }^{[14]}$ The optical response can be tuned by changing external field, such as electric and magnetic field. ${ }^{[15]}$ Aligning and patterning of CNWs with the aim of obtaining unique properties is also of particular interest for designing and creating advanced materials. ${ }^{[16]}$ In comparison, the feasibility of needle-like CNWs to form helical fibers through self-assembly has not been reported before. These helical fibers based on surface-acylated CNWs 
were systematically analyzed using diverse methods, and the formation mechanism was investigated by varying the critical parameters, including the wettability of substrate, the dispersant solvent, the amount of 10-undecenoyl groups, the crystallinity, the dimension of CNWs and the length of acyl chains.

CNWs with surface-immobilized 10-undecenoyl groups (CNWs-SU) were synthesized from microcrystalline cellulose (MCC) after the surface esterification by 10 -undecenoyl chloride under heterogeneous conditions and separated via ultracentrifugation (Figure 1a). The contents of surfaceimmobilized 10-undecenoyl groups were determined to be on average $3.52 \mathrm{mmolg}^{-1}$ based on the elemental analysis, which is corresponds to a degree of substitution (DS) of 1.32 . This product is thus referred to as $\mathrm{CNWs}^{-\mathrm{SU}_{1.32}}$ and they have typical whisker shapes as displayed by TEM images (Figure $1 \mathrm{~b}$ ). They have an average length of $135 \pm 37 \mathrm{~nm}$ and an average diameter of $4 \pm 1 \mathrm{~nm}$ based on the measurements on 100 single CNWs.

Solid-state ${ }^{13} \mathrm{C}$ CP-MAS NMR and FTIR spectra of MCC and $\mathrm{CNWs}-\mathrm{SU}_{1.32}$ further confirmed the chemical compositions and were used for the determination of their crystallinities (Figure 1c, Figure S1 and S2 in the Supporting Information). The signals between 55 and $110 \mathrm{ppm}$ are ascribed to the carbons of anhydroglucose units of cellulose. Among them, the signal at $88.5 \mathrm{ppm}$ is attributed to $\mathrm{C} 4$ in crystalline regions and the signal at $83.7 \mathrm{ppm}$ arises from the C4 in amorphous regions. ${ }^{[17]}$ After the esterification, new signals in addition to resonances between 110 and $55 \mathrm{ppm}$ emerged within the spectrum of CNWs-SU $\mathrm{SU}_{1.32}$. The signals between 40 and $10 \mathrm{ppm}$ are attributed to the carbon atoms of the aliphatic groups and the peak at $173 \mathrm{ppm}$ is ascribed to the carbon from the ester linkages. The signals at 114 and $139 \mathrm{ppm}$ are attributed to carbons in terminal olefin groups. Based on the signals ascribed to $\mathrm{C} 4$ in crystalline (88.5 ppm) and amorphous (83.7 ppm) domains, the crystallinity of MCC is determined to be approximately $58 \%$ and that of CNWs$\mathrm{SU}_{1.32}$ is about $40 \%$ (refer to Figure S3a and S3b). Because the modification of MCC generally proceeds from outer layers to the interior, 10 -undecenoyl moieties in $\mathrm{CNWs}^{-\mathrm{SU}_{1.32}}$ are present within a shell in the outer layers around the crystalline cellulose core (Figure 1a). Due to these acyl groups within the outer shells. CNWs-SU $\mathrm{CH}_{1.32}$ were well dispersible in diverse organic solvents, such as THF and dichloromethane (DCM).

After depositing the THF suspensions of CNWs-SU $\mathrm{CH}_{1.32}$ on the surface of silicon wafers, CNWs-SU $\mathrm{CH}_{1.32}$ gradually selfassembled into helical fibers during drying in the air under ambient conditions (Figure 1a,d,e). These integrated helical structures can reach a length of 5-6 $\mu \mathrm{m}$ and contain smaller fibers with a length between 1-2 $\mu \mathrm{m}$ (Figure $1 \mathrm{~d}$ ). The smaller fibers are generally twisted and composed of several single $\mathrm{CNWs}_{1.32}$. Moreover, the helices in these helical fibers are generally left-handed (Figure $1 \mathrm{e}$ ).

For comparison, a polymeric cellulose 10-undecenoyl ester with a DS of 1.10 (referred to as $\mathrm{PCUE}_{1.1}$ ) that was synthesized under homogeneous conditions did not form helical fibers under otherwise equal conditions, as shown in Figure S4a. In addition to the $\mathrm{PCUE}_{1.1}$, polymeric cellulose 10 -undecenoyl ester and cellulose stearoyl ester with complete derivatization of hydroxy groups and thus DS of 3, referred to as $\mathrm{CUE}_{3}$ and $\mathrm{CSE}_{3}$, also did not form helical fibers under similar conditions (refer to Figure S4b and S4c). Thus,
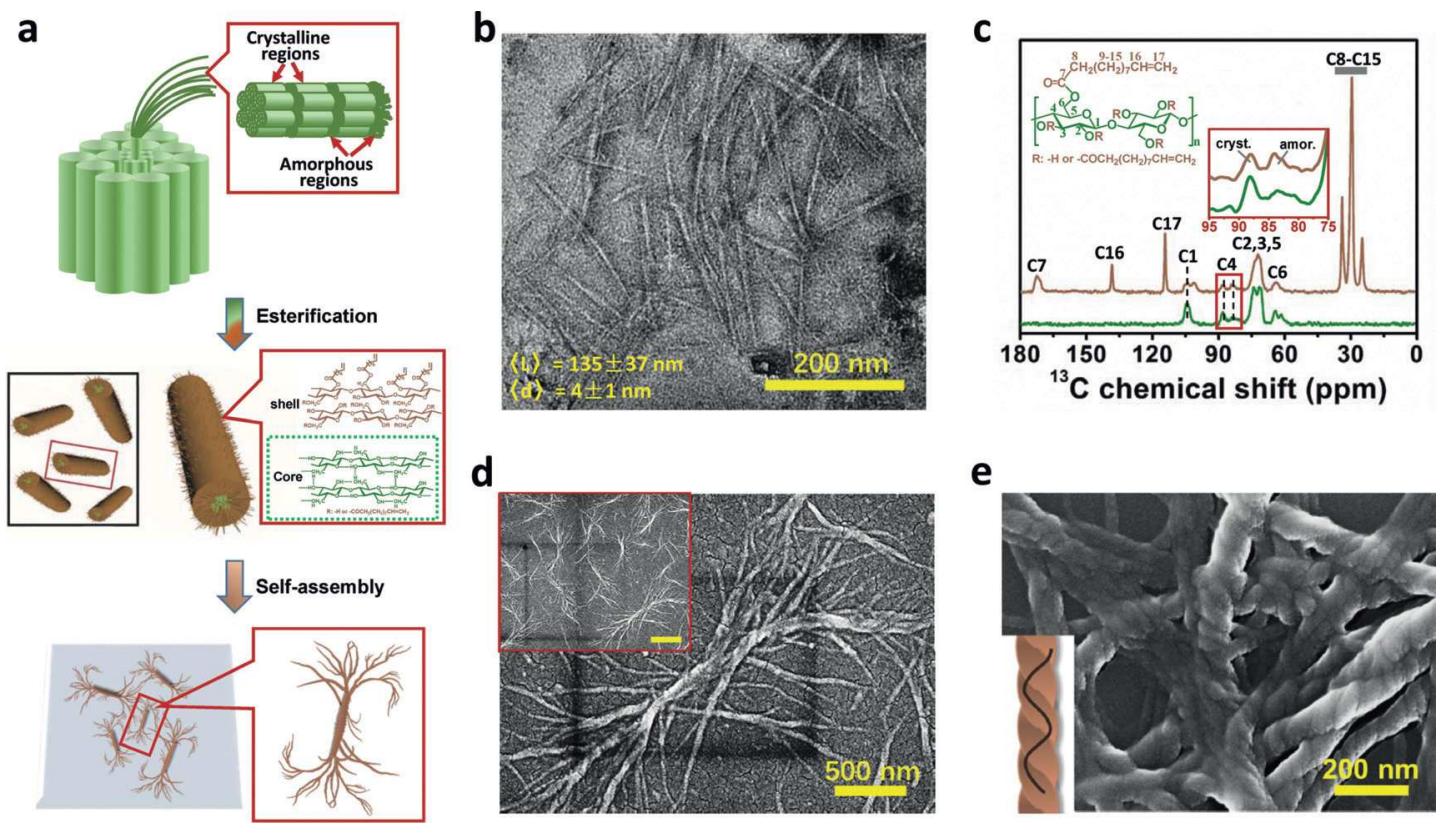

Figure 1. a) Schematic illustration for the synthesis of $\mathrm{CNWs}-\mathrm{SU}_{1.32}$ and further formation of self-assembled helical fibers driven by solvent evaporation. b) A representative TEM image of CNWs-SU ${ }_{1.32}$. c) Solid-state ${ }^{13} \mathrm{C}$ CP-MAS NMR spectra of microcrystalline cellulose, and CNWs$\mathrm{SU}_{1.32}$. d) A typical SEM image of helical fibers constructed by $\mathrm{CNWs}-\mathrm{SU}_{1.32}$. The inset shows more typical helical fibers with the scale bar of $1 \mu$ m. e) A representative SEM image of helical fibers constructed by $\mathrm{CNWS}_{\mathrm{S}} \mathrm{SU}_{1.32}$ showing details of the helix. The inset shows a schematic illustration of the left-handed helical structure. 
the presence of rigid $\mathrm{CNWs}$ as the crystalline core is a determining factor for the formation of helical fibers.

AFM images of self-assembled CNWs-SU $\mathrm{C}_{1.32}$ showed the formation of long bundles by twisted fibers with the lengths of several micrometers. Both the bundles and twisted fibers contained well-defined left-handed helical structures (Figure $2 \mathrm{a}, \mathrm{b})$.

The heights of single fibers range from 3 to $8 \mathrm{~nm}$, as shown by the height profiles across the marked sections (Figure $2 \mathrm{~b}, \mathrm{c}, \mathrm{d})$. The average height of these single fibers of $5.29 \pm 1.02 \mathrm{~nm}$ is similar as the result of TEM image of single $\mathrm{CNWs} \mathrm{SU}_{1.32}$ (Figure $1 \mathrm{~b}$ and Figure $2 \mathrm{~d}$ ). Thus, single CNWs$\mathrm{SU}_{1.32}$ are obviously the structural units for further construction of twisted fibers, which generally contain several single $\mathrm{CNWs}_{\mathrm{S}} \mathrm{SU}_{1.32}$ (yellow arrows in Figure $2 \mathrm{~b}$ ). The heights of twisted fibers lie between 10 and $20 \mathrm{~nm}$ (Figure $2 \mathrm{e}, \mathrm{f}$ ). This indicates the presence of three or four individual CNWs$\mathrm{SU}_{1.32}$ within one twisted helical fiber (white arrows in Figure 2b). Furthermore, at least two to five of such branches formed the backbone of the helical fiber bundles, based on the diameters of bundles around $50 \mathrm{~nm}$ (green arrow in Figure $2 \mathrm{~b}, \mathrm{e})$.
In addition to optically visible pitches (Figure $2 \mathrm{~g}$ ), fluctuations in height along the fibers provided further evidence of helical structures of the fibers. The height along the fiber axis revealed a zigzag pattern with the pitch length fluctuation between 40 and $140 \mathrm{~nm}$ (Figure 2h,i), while the average length of helical pitches was around $77 \mathrm{~nm}$. It is observed that the pitch lengths of the twisted fibers often increase with their heights as reported on other twisted fibers containing nanotubes and peptides. ${ }^{[18]}$ In addition, Hall et al. found the relationship between the optimal cross-sectional dimensions of bundles and the final equilibrium pitch. ${ }^{[19]}$

To understand the mechanisms about the self-assembly of CNWs-SU $\mathrm{SU}_{1.32}$ into helical fibers, we varied the critical parameters affecting the self-assembly process. Among others are the substrate wettability, dispersing solvent, content of surface-attached 10-undecenoyl groups, the crystallinity, the dimension of CNWs and the type of acyl groups with distinct chain lengths.

First, we varied the substrates and the dispersing solvents for the self-assembly process. With a static water contact angle of approximately $50^{\circ}$, bulk silicon wafers demonstrate as hydrophilic substrates (refer to Figure S5a). As hydrophobic substrates, both surface-hydrophobized silicon wafers with chloro(dimethyl)octadecylsilane (static water contact angles of $107 \pm 2^{\circ}$ ) and polydimethylsiloxane (PDMS, with static water contact angles of $120 \pm 2^{\circ}$ ) substrates were used to study the effect of substrate wettability on the self-assembly process (refer to Figure S5b and S5c).

In contrast to the helical structures on the silicon wafers, CNWs-SU $\mathrm{CU}_{1.32}$ did not form helical fibers on the surface of PDMS, but only aggregates. Single CNWs with their original needle-like shapes were maintained on PDMS (Figure $3 \mathrm{a}, \mathrm{b}$ ). The heights of the whiskers are between 3.5-4 nm (Figure 3c), which is approximately the same height as the diameters of single $\mathrm{CNWs}_{\mathrm{SU}} \mathrm{SU}_{1.32}$ measured via TEM measurements. In a similar way, no helical fiber was generated by $\mathrm{CNWs}-\mathrm{SU}_{1.32}$ on the surface of hydrophobized silicon wafer. Instead, CNWs-SU $\mathrm{CU}_{1.32}$ tended to aggregate into smooth fibers (refer to Figure S7). Therefore, CNWs-SU ${ }_{1.32}$ cannot self-assemble into helical structures on hydrophobic substrates under applied conditions. This is primarily due to the presence of surface-attached non-polar acyl chains. CNWs-SU $\mathrm{SU}_{1.32}$ should spread better on hydrophobic surfaces via the hydrophobic interaction between surface-attached acyl chains and hydrophobic surface. In comparison, $\mathrm{CNWs}_{-} \mathrm{SU}_{1.32}$ prefer to self-assembly with each other on hydrophilic surfaces.

Furthermore, the dispersant for CNWs$\mathrm{SU}_{1.32}$ was altered to examine the effect of the evaporation rates on the drying process, which further influences the self-assembly process. DCM and toluene were chosen as dispersant in
Figure 2. AFM analysis of helical fibers. AFM amplitude (a) and height (b) image of helical fibers formed on silicon wafer using the THF suspension of CNWs-SU $\mathrm{SU}_{1.32}$ at a concentration of $0.0002 \mathrm{mg} \mathrm{mL}^{-1}$. Individual $\mathrm{CNWs}^{-S_{1.32}}$ (yellow arrows) self-assembled into branches of the helical fiber bundles (white arrows) and further into the backbone of the helical fiber bundles (green arrows). c) Height profiles of single fibers 1, 2, and 3 indicated in (b). d) Height distribution of single fibers with the average height of single fibers: $h=5.29 \pm 1.02 \mathrm{~nm}$. e),f) Height profiles across assembled twisted fibers 4 and 5 indicated in (b). g) Magnified regions of the AFM image from (b) showing the detailed helical structures. The scale bar in the inset is $500 \mathrm{~nm}$. h) Height profile along the helical fiber showing the height fluctuation along the fiber axis and the helical pitch as displayed in (g). i) The distribution of the helical pitch length within helical pitches. The average length of helical pitches: $I=77.79 \pm 22.55 \mathrm{~nm}$. www.angewandte.de 
a

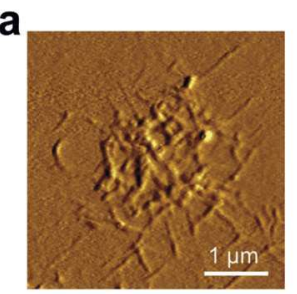

d

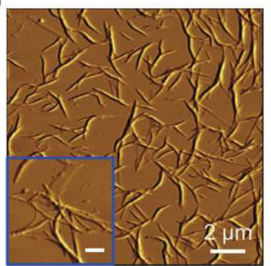

b

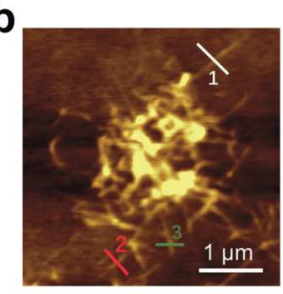

e

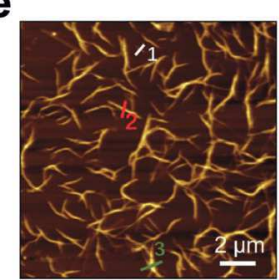

C

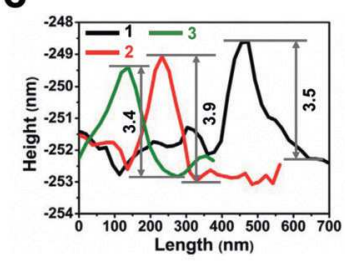

f

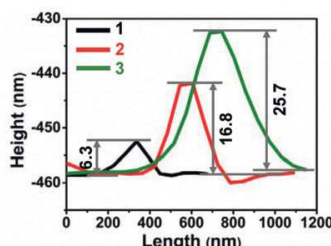

Figure 3. a) AFM amplitude and b) height image of dried $\mathrm{CNWs}-\mathrm{SU}_{1.32}$ on PDMS substrate using their THF suspensions at a concentration of $0.0002 \mathrm{mg} \mathrm{mL}^{-1}$. c) Height profiles of 1, 2, and 3 shown in (b). d) AFM amplitude and e) height image of dried $\mathrm{CNW}_{\mathrm{s}} \mathrm{SU}_{1.32}$ on silicon wafer using their DCM suspensions at a concentration of $0.0002 \mathrm{mg} \mathrm{mL}^{-1}$. The scale bar in the inset is $500 \mathrm{~nm}$. f) Height profiles of 1, 2, and 3 shown in (e).

addition to THF, because DCM has a lower boiling point $\left(39.6^{\circ} \mathrm{C}\right)$ and toluene has a higher boiling point $\left(111^{\circ} \mathrm{C}\right)$ than THF $\left(66^{\circ} \mathrm{C}\right)$ under atmospheric pressure. Thus, DCM will evaporate faster than THF under ambient conditions, while toluene slower. $10 \mu \mathrm{L}$ dispersion of $\mathrm{CNWs}^{-\mathrm{SU}_{1.32}}$ in $\mathrm{DCM}$, THF, and toluene on silicon wafer needed until total dry $49 \pm$ $4,159 \pm 15$ and $446 \pm 20$ seconds, respectively. By using the DCM dispersion of CNWs-SU ${ }_{1.32}$, most CNWs-SU ${ }_{1.32}$ assembled into rod-like fibers with the height of up to ten nanometers and the length of approximately $2 \mu \mathrm{m}$ (Figure $3 \mathrm{~d}-\mathrm{f}$ ). A part of them even assembled into relatively long fibers with the length of 4-5 $\mu \mathrm{m}$ and the height of dozens of nanometers. In contrast, by using toluene as dispersant, most of CNWs-SU $\mathrm{CU}_{1.32}$ aggregated irregularly together after drying on silicon wafers (refer to Figure S8). We obtained different structures by using distinct solvents, that is, THF, DCM and toluene, which demonstrates the critical effect of solvent evaporation on the self-assembly process. THF with an intermediate evaporation rates under atmospheric conditions provided sufficient time for $\mathrm{CNWs} \mathrm{SU}_{1.32}$ to self-assemble into helical fibers. In comparison, faster evaporation of DCM did not assure sufficient time for CNWs-SU $\mathrm{CH}_{1.32}$ to selfassembly, while only single short fibers of aggregated CNWs-SU $S_{1.32}$ were obtained. Toluene with a much lower evaporation rate only led to strong aggregation due to the continuous accumulation of $\mathrm{CNWs}_{\mathrm{S}} \mathrm{SU}_{1.32}$.

Diverse CNWs-SU with various amounts of 10-undecenoyl groups and crystallinities were prepared by varying the reaction time, the dosage of acid chloride or the type of reaction medium. $\mathrm{CNWs}^{-\mathrm{SU}_{0.98}}$ with a DS of 0.98 was obtained by using $1.5 \mathrm{~mol} 10$-undecenoyl chloride per mol anhydroglucose units of cellulose (Figure $4 \mathrm{a}$ ). They have an average length of $97 \pm 24 \mathrm{~nm}$ and an average diameter of $4 \pm$ $0.6 \mathrm{~nm}$. Based on solid-state ${ }^{13} \mathrm{C} \mathrm{CP}-\mathrm{MAS}$ NMR spectra, the crystallinity of CNWs-SU $\mathrm{CU}_{0.98}$ is determined to be approximately $41 \%$, which is similar as that of $\mathrm{CNWs}^{-\mathrm{SU}_{1.32}}$ (refer to

Figure S3c). CNWs-SU 0.98 self-assembled after drying on silicon wafers into dendroid fibers, while just part of fibers with helical structure (Figure $4 \mathrm{a}$ ). By changing reaction times to 3 and $20 \mathrm{~h}$, two other CNWs-SU with DSs of 1.15 and 1.66 were synthesized, referred to as $\mathrm{CNWs}^{-\mathrm{SU}_{1.15}}$ and $\mathrm{CNWs}-\mathrm{SU}_{1.66}$, respectively. They have similar morphologies as $\mathrm{CNWs-SU_{1.32 }}$ and $\mathrm{CNWs}-\mathrm{SU}_{0.98}$ but with slightly different dimensions (Figure $4 \mathrm{~b}, \mathrm{c})$. CNWs-SU $\mathrm{CN}_{1.15}$ have an average length of $113 \pm 20 \mathrm{~nm}$ and an average diameter of $4 \pm$ $0.5 \mathrm{~nm}$, while $\mathrm{CNWs}^{-\mathrm{SU}_{1.66}}$ have an average length of $86 \pm 12 \mathrm{~nm}$ and an average diameter of $3.5 \pm 0.5 \mathrm{~nm}$. The crystallinity of CNWs-SU $\mathrm{SU}_{1.15}$ and $\mathrm{CNWs} \mathrm{SU}_{1.66}$ is determined to be approximately $54 \%$ and $37 \%$ according to solid-state ${ }^{13} \mathrm{C} \mathrm{CP}$ MAS NMR spectra, respectively (refer to Fig-

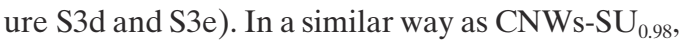
both CNWs-SU $\mathrm{CU}_{1.15}$ and CNWs-SU $\mathrm{C}_{1.66}$ could selfassemble into long fibers with partially helical structures (Figure 4b,c).

Thus, the DSs of diverse CNWs-SU play a critical role for the generation of explicit lefthanded helical fibers via self-assembly, while the crystallinity of diverse CNWs-SU affects the formation of helical fibers to a certain extent due to their association with the alternating DSs during the reaction. Too low or too high amounts of surface-attached 10-undecenoyl groups are not preferable for the self-assembly of helical fibers, while an intermediate amount of undecenoyl groups is more suitable. Moreover, $\mathrm{CNWs} \mathrm{SU}_{1.32}$ with the longest $\mathrm{CNWs}$ preferentially tended to form explicit helical fibers, while the other CNWs-SU (CNWs-SU ${ }_{0.98}, \mathrm{CNWs} \mathrm{SU}_{1.15}$ and CNWs-SU ${ }_{1.66}$ ) with similar diameters and crystallinities only partially form helical fibers.

We further explored the effects of acyl chain lengths on the self-assembly. CNWs with surface-attached hexanoyl groups (CNWs-SH $\left.{ }_{1.57}\right)$ or stearoyl groups $\left(\mathrm{CNWs-S_{1.59 }}\right)$ were synthesized correspondingly. Both $\mathrm{CNWs}^{-\mathrm{SH}_{1.57}}$ and

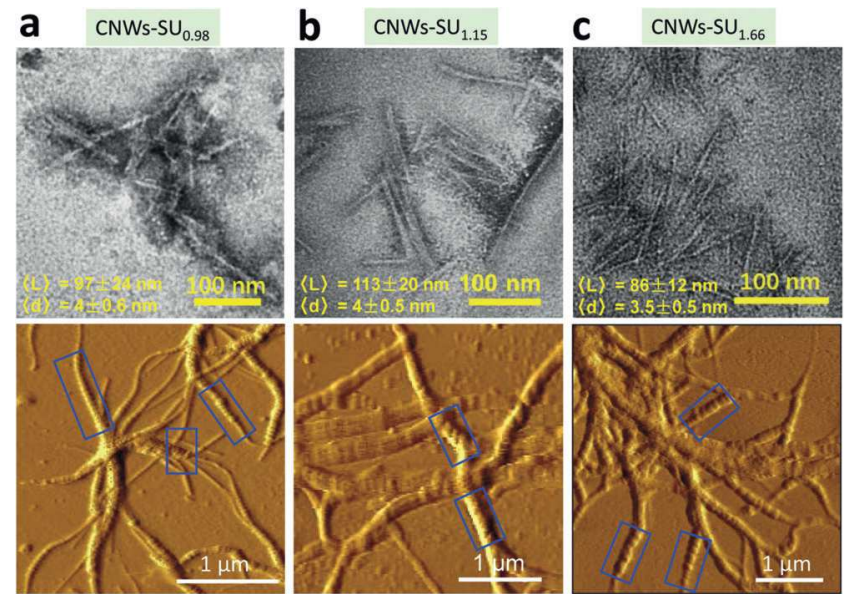

Figure 4. Representative TEM images of and AFM amplitude images of diverse CNWs-SU on silicon wafers using their THF suspensions at a concentration of $0.0002 \mathrm{mg} \mathrm{mL}^{-1}$ : a) $\mathrm{CNWs}-\mathrm{SU}_{0.98}$, b) $\mathrm{CNWs}_{\mathrm{S}} \mathrm{SU}_{1.15}$, and c) $\mathrm{CNWs}-\mathrm{SU}_{1.66}$. 
$\mathrm{CNWs} \mathrm{SS}_{1.59}$ could not self-assemble into significant helical fibers, but only formed long fibers with periodic rough surface patterns during the drying process (refer to Figure S9c and S9d). Due to the presence of high amounts of modified surface layers containing hexanoyl or stearoyl moieties, $\mathrm{CNWs} \mathrm{SH}_{1.57}$ and $\mathrm{CNWs} \mathrm{SS}_{1.59}$ had a crystallinity of only $23 \%$ and $22 \%$ (refer to Figure S3g and S3h), respectively. It should be noted that the CNWs-SS with a lower DS was not possible, while a harsh modification of cellulose fibers was accompanied by a lower crystallinity. Instead, $\mathrm{CNWs}^{-\mathrm{SH}_{1.37}}$ with a lower DS ascribed to hexanoyl groups of 1.37 was synthesized, but $\mathrm{CNWs}-\mathrm{SH}_{1.37}$ could not self-assemble into helical fibers and only resulted in long fibers with rough surfaces (refer to Figure S10). Thus, the acyl chain lengths strongly influence the formation of helical fibers, while the 10undecenoyl groups are the most appropriate acyl chains compared to hexanoyl and stearoyl moieties.

In summary, the results show the significant effects of the wettability of substrate, dispersing solvents, the amount of 10undecenoyl groups, the crystallinity, the length of CNWs and the length of acyl chains on the self-assembly process driven by the solvent evaporation (Figure 5). These parameters serve to adjust the self-assembly process and the final resulting helical structures. Such helical structures can endow materials with many excellent properties for potentially broad applications. Moreover, the proposed self-assembly approach would open up a promising avenue for growing and patterning nanostructures using surface-functionalized nanocellulose.

\section{Acknowledgements}

K.Z. thanks German Research Foundation (DFG) for the financial support for the project (Project Nr. ZH546/2-1) and Georg-August-University of Goettingen for the start-up funding. H.L. and B.P. and thank the Chinese Scholarship Council (CSC) for the financial support. We thank Prof. Dr. Christoph F. Schmidt from the Third Institute of PhysicsBiophysics, Georg-August-University of Goettingen for the support by AFM measurements.

\section{Conflict of interest}

The authors declare no conflict of interest.

Keywords: acylation - cellulose - helical fibers - nanowhiskers · self-assembly

How to cite: Angew. Chem. Int. Ed. 2018, 57, 16323-16328 Angew. Chem. 2018, 130, 16561-16566

[1] S. Isnard, W. K. Silk, Am. J. Bot. 2009, 96, 1205-1221.

[2] a) S. Yang, L. Zhao, C. Yu, X. Zhou, J. Tang, P. Yuan, D. Chen, D. Zhao, J. Am. Chem. Soc. 2006, 128, 10460 -10466; b) Q. Guo, Z. Chen, W. Li, P. Dai, K. Ren, J. Lin, L. A. Taber, W. Chen, Europhys. Lett. 2014, 105, 64005.

[3] R. Langridge, W. E. Seeds, H. R. Wilson, C. W. Hooper, M. H. F. Wilkins, L. D. Hamilton, J. Biophys. Biochem. Cytol. 1957, 3, $767-778$.

[4] A. N. Lupas, M. Gruber, Adv. Protein Chem. Vol. 70, Academic Press, 2005, pp. $37-38$.

[5] T. Muraoka, H. Cui, S. I. Stupp, J. Am. Chem. Soc. 2008, 130, 2946-2947.

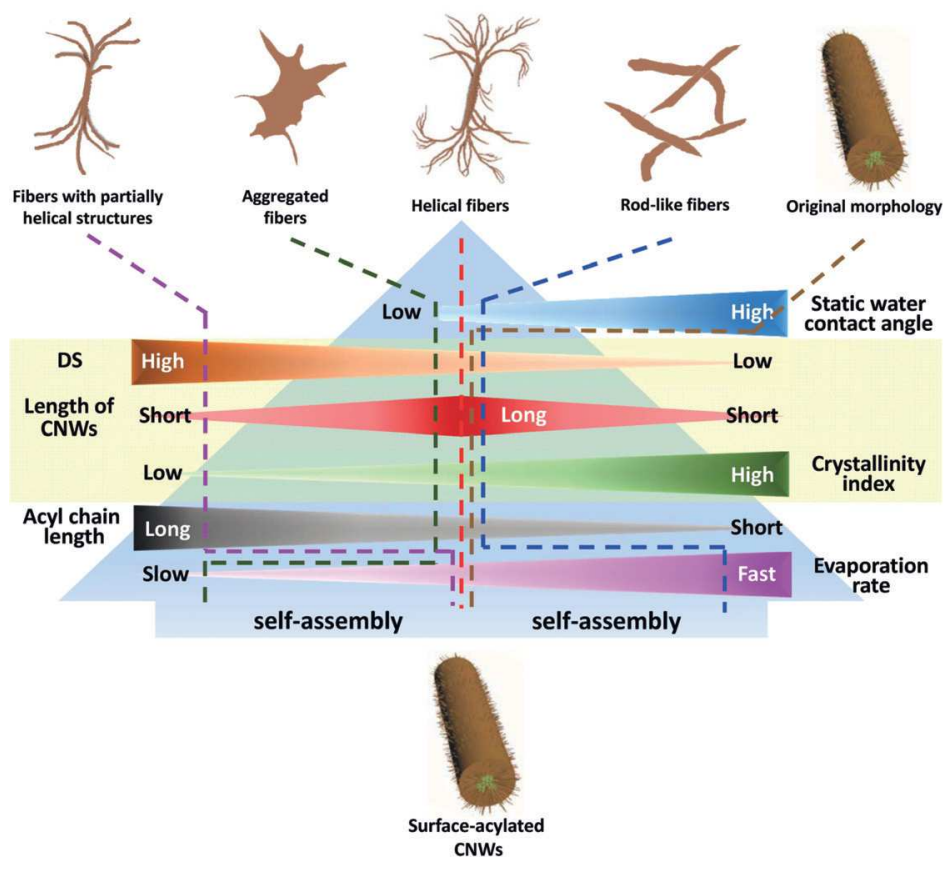

[6] a) D. R. Eyre, Science 1980, 207, 1315-1322; b) K. Okuyama, M. Takayanagi, T. Ashida, M. Kakudo, Polym. J. 1977, 9, 341; c) K. Watanabe, J. Nakagawa, T. Ebihara, Y. Okamoto, Polymer 1997, 38, 5155-5159.

[7] a) G. Whitesides, J. Mathias, C. Seto, Science 1991, 254, $1312-1319$; b) G. M. Whitesides, B. Grzybowski, Science 2002, 295, 2418-2421; c) M. Grzelczak, J. Vermant, E. M. Furst, L. M. Liz-Marzán, ACS Nano 2010, 4, 3591-3605; d) Z. Nie, A. Petukhova, E. Kumacheva, Nat. Nanotechnol. 2010, 5, 15-25; e) Q. Wang, Z. Wang, Z. Li, J. Xiao, H. Shan, Z. Fang, L. Qi, Sci. Adv. 2017, 3, 2375-2548.

[8] a) C. C. Lee, C. Grenier, E. W. Meijer, A. P. H. J. Schenning, Chem. Soc. Rev. 2009, 38, 671-683; b) F. J. M. Hoeben, P. Jonkheijm, E. W. Meijer, A. P. H. J. Schenning, Chem. Rev. 2005, 105, $1491-$ 1546.

[9] G. Nyström, M. Arcari, R. Mezzenga, Nat. Nanotechnol. 2018, 13, 330-336.

[10] a) T. P. J. Knowles, R. Mezzenga, Adv. Mater. 2016, 28, 6546-6561; b) C. Li, A.-K. Born, T. Schweizer, M. Zenobi-Wong, M. Cerruti, R. Mezzenga, Adv. Mater. 2014, 26, 3207-3212; c) C. Li, J. Adamcik, R. Mezzenga, Nat. Nanotechnol. 2012, 7, 421-427.

[11] a) P. Chen, Y. Xu, S. He, X. Sun, S. Pan, J. Deng, D. Chen, H. Peng, Nat. Nanotechnol. 2015, 10, 1077-1083; b) H. Engelkamp, S. Middelbeek, R. J. M. Nolte, Science 1999, 284, 785-788.

acylated CNWs affected by diverse parameters during the self-assembly.

[12] M. A. S. Azizi Samir, F. Alloin, A. Dufresne, Biomacromolecules 2005, 6, 612-626. 
[13] M. M. de Souza Lima, R. Borsali, Macromol. Rapid Commun. 2004, 25, $771-787$.

[14] a) G. Guidetti, S. Atifi, S. Vignolini, W. Y. Hamad, Adv. Mater. 2016, 28, 10042-10047; b) K. E. Shopsowitz, H. Qi, W. Y Hamad, M. J. MacLachlan, Nature 2010, 468, 422-425; c) A. G. Dumanli, H. M. van der Kooij, G. Kamita, E. Reisner, J. J. Baumberg, U. Steiner, S. Vignolini, ACS Appl. Mater. Interfaces 2014, 6, 12302-12306.

[15] a) B. Frka-Petesic, H. Radavidson, B. Jean, L. Heux, Adv. Mater. 2017, 29, 1606208; b) B. Frka-Petesic, G. Guidetti, G. Kamita, S Vignolini, Adv. Mater. 2017, 29, 1701469.

[16] M. Mashkour, T. Kimura, F. Kimura, M. Mashkour, M. Tajvidi, Biomacromolecules 2014, 15, 60-65.
[17] A. Isogai, M. Usuda, T. Kato, T. Uryu, R. H. Atalla, Macromolecules 1989, 22, 3168-3172.

[18] a) L. Ziserman, H.-Y. Lee, S. R. Raghavan, A. Mor, D. Danino, J. Am. Chem. Soc. 2011, 133, 2511-2517; b) M. Wang, P. Zhou, J. Wang, Y. Zhao, H. Ma, J. R. Lu, H. Xu, J. Am. Chem. Soc. 2017, 139, 4185-4194.

[19] D. M. Hall, I. R. Bruss, J. R. Barone, G. M. Grason, Nat. Mater. 2016, $15,727-732$.

Manuscript received: July 18, 2018

Accepted manuscript online: September 27, 2018

Version of record online: November 11, 2018 


\section{Angewandte}

Supporting Information

Helical Fibers via Evaporation-Driven Self-Assembly of SurfaceAcylated Cellulose Nanowhiskers

Huan Liu, Bo Pang, Renata Garces, Riza Dervisoglu, Longquan Chen, Loren Andreas, and Kai Zhang*

ange_201808250_sm_miscellaneous_information.pdf 


\section{Author Contributions}

H.L. Data curation: Lead; Investigation: Lead; Methodology: Lead; Visualization: Lead; Writing—original draft: Lead

B.P. Investigation: Supporting; Methodology: Supporting; Writing—review \& editing: Supporting

R.G. Methodology: Supporting; Writing_review \& editing: Supporting

R.D. Methodology: Supporting; Writing—review \& editing: Supporting

L.C. Methodology: Supporting; Writing—review \& editing: Supporting

L.A. Methodology: Supporting; Writing-review \& editing: Supporting

K.Z. Conceptualization: Lead; Funding acquisition: Lead; Project administration: Lead; Resources: Lead; Supervision: Lead; Visualization: Supporting; Writing—original draft: Supporting; Writing—review \& editing: Lead. 


\section{Table of Contents}

1 General information

2 Experimental Part
2.1 Synthesis Description

2.2 Hydrophobization of Silicon Wafers

2.3 Preparation of Polydimethylsiloxane (PDMS) Substrates

2.4 Elemental Analysis

2.5 Solid-State ${ }^{13} \mathrm{C}$ CP-MAS NMR Spectroscopy

2.6 Scanning Electron Microscopy (SEM)

2.7 Transmission Electron Microscope (TEM)

2.8 Atomic Force Microscopy (AFM)

2.9 Static Water Contact Angles

3 Results and Discussion

4 References

5 Author Contributions 


\section{General information}

Microcrystalline cellulose (MCC) with an average particle size of $50 \mu \mathrm{m}, 10$-undecenoyl chloride, stearoyl chloride and hexanoyl chloride were purchased from Sigma-Aldrich Chemie $\mathrm{GmbH}$ (Steinheim, Germany). MCC was dried in vacuum oven for 24 hours at $80{ }^{\circ} \mathrm{C}$ before use. Dry pyridine and tetrahydrofuran (THF) were brought from Th. Geyer GmbH \& Co. KG (Renningen, Germany). Other chemicals are all of analytical grade and used as received. Deionized water (DI water) was used in all experiments.

\section{Experimental Part}

\subsection{Synthesis Description}

Synthesis of cellulose nanowhiskers with surface-attached 10-undecenoyl groups (CNWs-SU) and surface-attached hexanoyl cellulose nanowhiskers (CNWs-SH)

CNWs were synthesized under heterogeneous conditions according to published procedures with a few modifications. ${ }^{[1]}$ Briefly, $1 \mathrm{~g}$ of MCC was fed into a three-necked flask. Then, $30 \mathrm{~mL}$ pyridine was added and MCC dispersed therein by stirring. After the mixture was heated up to $50{ }^{\circ} \mathrm{C}, 2.73 \mathrm{~mL}$ or $2.05 \mathrm{~mL}$ 10-undecenoyl chloride $(2 \mathrm{~mol}$ or $1.5 \mathrm{~mol}$ acid chloride per mol anhydroglucose units of cellulose) or 2.67 or $2.23 \mathrm{~mL}$ hexanoyl chloride ( $3 \mathrm{~mol}$ or $2.5 \mathrm{~mol}$ acid chloride per mol anhydroglucose units of cellulose) were added to the cellulose suspension under stirring. After 3,7 or $20 \mathrm{~h}$ stirring at $50{ }^{\circ} \mathrm{C}$, the reaction mixture was poured into $200 \mathrm{~mL}$ methanol immediately. The precipitate was separated by centrifugation. Thereafter, the product was purified by a repeated dispersion and precipitation process using THF and methanol, respectively, before the product was dispersed in THF. Then, the suspension was centrifuged for $20 \mathrm{~min}$ at $14000 \mathrm{rpm}$ at $4{ }^{\circ} \mathrm{C}$. After the centrifugation, the supernatant was removed and the solid was dispersed in THF again, which was centrifuged for $20 \mathrm{~min}$ at $3000 \mathrm{rpm}$ at $20^{\circ} \mathrm{C}$ to remove the micro-scaled segments. Finally, the purified product was separated on ultracentrifuge at $14000 \mathrm{rpm}$ at $4{ }^{\circ} \mathrm{C}$ for $10 \mathrm{~h}$, leading the corresponding CNWs with the yields of $11.6 \pm 2.4 \%$. The DS of CNWs-SU under the conditions of $2 \mathrm{~mol} 10$-undecenoyl chloride per mol anhydroglucose units of cellulose for 3,7 and $20 \mathrm{~h}$ is $1.15,1.32$ and 1.66, namely $\mathrm{CNWs}-\mathrm{SU}_{1.15}, \mathrm{CNWs}-\mathrm{SU}_{1.32}$ and $\mathrm{CNWs}-\mathrm{SU}_{1.66}$, respectively. With the condition of 1.5 mol 10 -undecenoyl chloride per mol anhydroglucose units of cellulose for $7 \mathrm{~h}$, CNWs-SU with the DS of 0.98 (named as $\mathrm{CNWs} \mathrm{SU}_{0.98}$ ) was obtained The degree of substitution of CNWs-SH synthesized with 3 and $2.5 \mathrm{~mol}$ hexanoyl chloride per mol anhydroglucose units of cellulose for $7 \mathrm{~h}$ was determined to be 1.57 and 1.37, respectively. The samples were referred to as $\mathrm{CNWs}^{-\mathrm{SH}_{1.57}}$ and $\mathrm{CNWs}^{-\mathrm{SH}_{1.37}}$

Synthesis of cellulose nanowhiskers with surface-attached stearoyl groups (CNWs-SS) in a mixed solvent of N,N-dimethylacetamide (DMAc) and pyridine

$1 \mathrm{~g} \mathrm{MCC}$ was fed into a three-necked flask. Then, $30 \mathrm{~mL}$ DMAc and $2.5 \mathrm{~mL}$ pyridine was added and MCC dispersed therein by stirring. The mixture was heated up to $100^{\circ} \mathrm{C}$ and $5.76 \mathrm{~mL}$ stearoyl chloride $(2.5 \mathrm{~mol}$ stearoyl chloride per mol anhydroglucose units of cellulose) was added to the hot cellulose suspension under stirring. After $1 \mathrm{~h}$ stirring at $100{ }^{\circ} \mathrm{C}$, the hot reaction mixture was poured into $200 \mathrm{~mL}$ ethanol immediately. The precipitate was separated by centrifugation. Thereafter, the product was purified by a repeated dispersion and precipitation process using THF and ethanol, respectively, before the product was dispersed in THF. Then, the suspension was centrifuged for $20 \mathrm{~min}$ at $14000 \mathrm{rpm}$ at $4{ }^{\circ} \mathrm{C}$. After the centrifugation, the supernatant was removed and the solid was dispersed in THF again, which was centrifuged for $20 \mathrm{~min}$ at $3000 \mathrm{rpm}$ at $20^{\circ} \mathrm{C}$ to remove the micro-scaled segments. Finally, the purified product was dispersed in THF for further use. The degree of substitution of CNWs-SS is 1.66 and the product is referred to as $\mathrm{CNWs}_{\mathrm{SS}}$ 1.66.

\section{Synthesis of polymeric cellulose 10-undecenoyl ester (PCUE) under homogeneous condition}

Homogeneous synthesis was carried out according to reference with a few modifications. ${ }^{[2]}$ In brief, $1 \mathrm{~g} \mathrm{MCC}$ and $40 \mathrm{~mL}$ DMAc was added into a three-necked flask and the mixture was heated up to $130^{\circ} \mathrm{C}$ for 30 min under rigorous stirring before $3 \mathrm{~g} \mathrm{LiCl}$ was added. The system was purged with nitrogen for at least $3 \mathrm{~min}$. Then, the mixture was cooled down to room temperature under stirring for additional 3 hours, until a transparent cellulose solution was obtained. After that, the above cellulose solution was heated up to $50{ }^{\circ} \mathrm{C}$ and $2.73 \mathrm{~mL}$ of 10-undecenoyl chloride (2 mol 10-undecenoyl chloride per mol anhydroglucose units of cellulose) was added. Thereafter, $3.25 \mathrm{~mL}$ pyridine was added ( $3 \mathrm{~mol}$ pyridine per mol acid chloride) under stirring. The mixture was then stirred for additional $3 \mathrm{~h}$ at $50{ }^{\circ} \mathrm{C}$ and the reaction was stopped by adding the warm mixture into 5 volumes of methanol. Then, the product was purified by a repeated solubilization and precipitation process using THF and methanol, respectively, before the product was dispersed in THF for further use. The degree of substitution is 1.1 and the product is named as PCUE $\mathrm{P}_{1.1}$.

\section{Synthesis of cellulose 10-undecenoyl ester with a degree of substitution of 3 ( $\mathrm{CUE}_{3}$ )}

$\mathrm{CUE}_{3}$ was prepared according to our previous work with a few modifications. ${ }^{[3]}$ In a typical case, $1 \mathrm{~g}$ dried MCC was fed into a threenecked flask. Then, $30 \mathrm{~mL}$ pyridine was added and MCC was dispersed therein by stirring. The cellulose suspension was heated up to $100{ }^{\circ} \mathrm{C}$ and $8.4 \mathrm{~mL} 10$-undecenoyl acid chloride (6 mol 10-undecenoyl chloride per mol anhydroglucose units of cellulose) was added into the hot cellulose suspension. After $1 \mathrm{~h}$ stirring at $100{ }^{\circ} \mathrm{C}$, the mixture was added into $200 \mathrm{~mL}$ methanol. The precipitate was separated by centrifugation. Thereafter, the product was purified by a repeated solubilization and precipitation process using THF and methanol, respectively. Finally, the product was dissolved in THF and stored in the dark for further use. 
Synthesis of cellulose stearoyl ester with a degree of substitution of $3\left(\mathrm{CSE}_{3}\right)$

$\mathrm{CSE}_{3}$ was synthesized under heterogeneous conditions according to published procedures ${ }^{[2]}$ Briefly, $1 \mathrm{~g}$ dried MCC was fed into a three-necked flask. Then, $30 \mathrm{~mL}$ pyridine was added and MCC dispersed therein by stirring. The cellulose suspension was heated up to $100{ }^{\circ} \mathrm{C}$ and $13.83 \mathrm{~mL}$ of stearoyl chloride (6 mol stearoyl chloride per mol anhydroglucose of cellulose) was added into the cellulose suspension. After $1 \mathrm{~h}$ stirring at $100{ }^{\circ} \mathrm{C}$, the reaction mixture was poured into $200 \mathrm{~mL}$ ethanol. The precipitate was separated by centrifugation. Thereafter, the product was purified by repeated dissolution in THF and precipitation in ethanol. Finally, the product was dried in vacuum at $50{ }^{\circ} \mathrm{C}$ for $12 \mathrm{~h}$.

\subsection{Hydrophobization of Silicon Wafers}

The hydrophobic silicon wafers were modified with chloro(dimethyl)octadecylsilane solution. Before the silanization, silicon wafers were treated with a plasma cleaner (Zepto Plasma cleaner, Diener Electronic, Germany). Then, the wafers were immersed in chloro(dimethyl)octadecylsilane solution of $15 \mathrm{mg} / \mathrm{ml}$ pyridine at $50{ }^{\circ} \mathrm{C}$ for $24 \mathrm{~h}$. After that, the wafers were sonicated in THF for 10 $\mathrm{min}$, in ethanol for $10 \mathrm{~min}$ and dried in the air, leading to silicon wafers with (dimethyl)octadecylsilyl groups at the surface.

\subsection{Preparation of Polydimethylsiloxane (PDMS) Substrates}

PDMS prepolymer and curing agent (Sylguard 184, Sigma-Aldrich) were mixed at a ratio of 10:1, stirred thoroughly, and then degassed in a vacuum desiccator. The prepolymer mixture was poured into a polystyrene Petri dish of $1 \mathrm{~mm}$ thick (VWR International $\mathrm{GmbH}$, Darmstadt, Germany), degassed again in the vacuum desiccator, and cured in the oven at $80{ }^{\circ} \mathrm{C}$ for $1 \mathrm{~h}$. Following the curing, flat PDMS slices were peeled from the dishes and cut into $1 \mathrm{~cm} \times 1 \mathrm{~cm}$ slices.

\subsection{Elemental Analysis}

The contents of carbon and hydrogen were determined with an Elemental Analyser 4.1 vario EL III (Elementar, Germany). DS of corresponding groups was calculated according to the carbon contents, as the following formula.

$$
\mathrm{DS}=\frac{5.13766-11.5592 \times C \%}{0.996863 \times C \%-0.856277 \times n+n \times C \%}
$$

where $\mathrm{C} \%$ is the content of element carbon and $\mathrm{n}$ is the number of carbon atoms in the acyl moieties, respectively.

\subsection{Solid-State ${ }^{13} \mathrm{C}$ CP-MAS NMR Spectroscopy}

Solid-state ${ }^{13} \mathrm{C}$ CP-MAS NMR measurements for all samples were acquired at ambient conditions using a Bruker AVANCE IIIHD 800WB spectrometer with a field strength of $18.8 \mathrm{~T}$. Experiments were performed using a Bruker $1.3 \mathrm{~mm}$ MAS triple resonance probe with a MAS spinning frequency of $55.555 \mathrm{kHz}$. A ramped CP-MAS sequence was used with a $1 \mathrm{H}$ excitation power of $138.8 \mathrm{kHz}$ and a Hartman Hahn contact time of $2 \mathrm{~ms}$ with a ${ }^{13} \mathrm{C}$ inherent radio frequency (rf) field strength of $62.5 \mathrm{kHz}$ and ${ }^{1} \mathrm{H}$ rf field strength of 127.4 $\mathrm{kHz}$. During acquisition of $27 \mathrm{~ms}$ a ${ }^{1} \mathrm{H}$ decoupling was performed with an inherent rf field of $14.7 \mathrm{kHz}$ employing WALTZ-16 sequence ${ }^{[4]}$ In a typical $8 \mathrm{~h}$ experiment, 15360 scans were accumulated with a recycle delay of $2 \mathrm{~s}$.

The crystallinities of the samples were determined based on the solid-state ${ }^{13} \mathrm{C} C P-M A S ~ N M R$ spectra. The individual peaks ascribed to $\mathrm{C} 4$ in crystalline and amorphous regions were extracted by a peak deconvolution process in Origin 8.5 using Gaussian function (Supplementary Fig. 3). The crystallinity was calculated using the following equation:

$$
\text { Crystallinity }(\%)=\frac{A_{\text {cr. }}}{A_{\text {cr. }+A_{a m}}}
$$

where $A_{c r}$ and $A_{a m}$. represent the areas obtained by the integration of the fitting curves of the crystalline peak and amorphous peaks in $\mathrm{C} 4$ region, respectively.

\subsection{Scanning Electron Microscopy (SEM)}

SEM images were obtained on a LEO Supra-35 high-resolution field emission scanning electron microscope (Carl Zeiss AG, Germany) at an accelerating voltage of $5 \mathrm{kV}$. A layer of carbon or gold was coated on the surface of samples before SEM measurements.

\subsection{Transmission Electron Microscope (TEM)}

The TEM observation was performed on a CM 12 Transmission Electron Microscope (Philips, Netherland). The specimen was stained by phosphotungstic acid solution ( $0.2 \mathrm{wt} \%$ in THF) for about $2 \mathrm{~min}$ to enhance the contrast.

\subsection{Atomic Force Microscopy (AFM)}

AFM topographic measurements were performed using a Cypher S AFM (Asylum Research, Santa Barbara, CA) in tapping mode in air. Samples were prepared by transferring a drop of diluted solution (concentration of $0.0002 \mathrm{mg} / \mathrm{mL}$.) onto different substrates followed by drying in air. Silicon Nitride cantilevers with a tip radius of $7 \mathrm{~nm}$, nominal spring constant of $2 \mathrm{~N} \mathrm{~m}^{-1}$, and nominal 
resonance frequency of $70 \mathrm{kHz}$ (AC240TS-R3, Olympus) were used. Images processing and subsequent evaluation of sample geometrical parameters was performed with Gwyddion (Free Software Foundation, Inc., Boston, USA).

\subsection{Static Water Contact Angles}

The wetting properties of substrates were assessed on a Contact Angle System OCA 15EC (dataphysics, Filderstadt, Germany). Static water contact angles were measured using the sessile drop $(4 \mu \mathrm{L})$ technique averaged over five repeats.

\section{Results and Discussion}

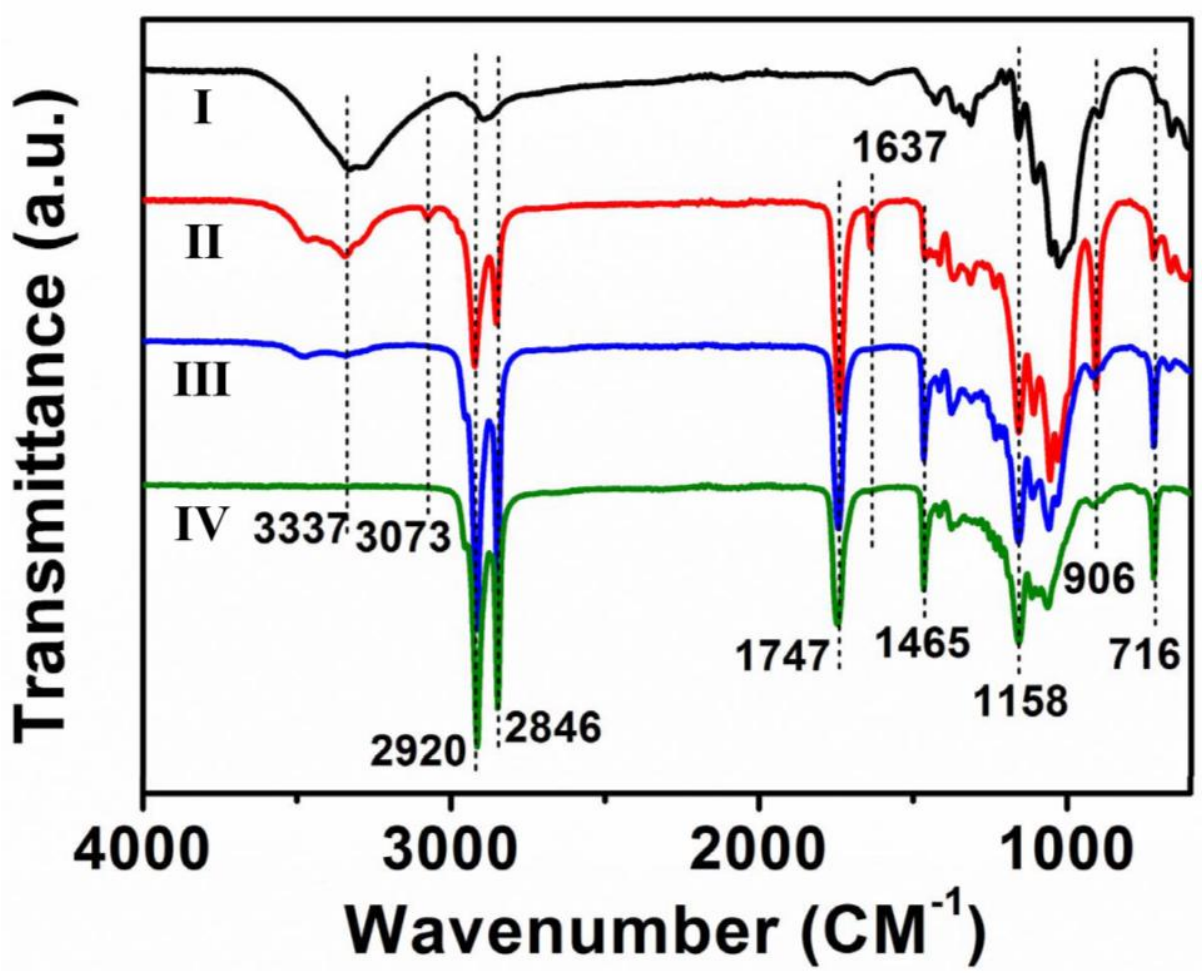

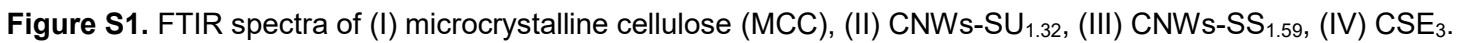

Typical FTIR bands of cellulose are visible, such as the bands attributed to vibrations of O-H (3000-3600 and $\left.1300-1450 \mathrm{~cm}^{-1}\right), \mathrm{C}-\mathrm{H}$ $\left(2900 \mathrm{~cm}^{-1}\right)$, and C-O groups $\left(950-1200 \mathrm{~cm}^{-1}\right)$.

Within the FTIR spectrum of CNWs-SU ${ }_{1.32}$, new bands emerge at $3073,1747,1637$ and $906 \mathrm{~cm}^{-1}$, which are attributed to stretching vibrations of $\mathrm{CH}_{2}=\mathrm{CH}_{2}$, stretching vibrations of $\mathrm{C}=\mathrm{O}$, stretching vibrations of $\mathrm{C}=\mathrm{C}$, and deformation vibrations of alkene $\mathrm{C}-\mathrm{H}$ groups, respectively.

Within the FTIR spectrum of CNWs-SS 1.59 and $\mathrm{CSE}_{3}$, new bands emerge at $1747,1465,1158$ and $716 \mathrm{~cm}^{-1}$, which are attributed to stretching vibrations of $\mathrm{C}=\mathrm{O}$, symmetric $\mathrm{C}-\mathrm{H}$ deformation vibrations, $\mathrm{C}-\mathrm{O}-\mathrm{C}$ stretching vibrations and $\mathrm{C}-\mathrm{C}$ rocking vibrations, respectively.

The intensities of signals at 2920 and $2846 \mathrm{~cm}^{-1}$ strongly increased, which are due to asymmetric and symmetric stretching vibrations of $\mathrm{C}-\mathrm{H}$ groups of alkyl chains, respectively.

The peak at $3337 \mathrm{~cm}^{-1}$ attributed to stretching vibrations of hydroxyl groups at cellulose backbone strongly decreased due to the derivatisation by acyl groups. 


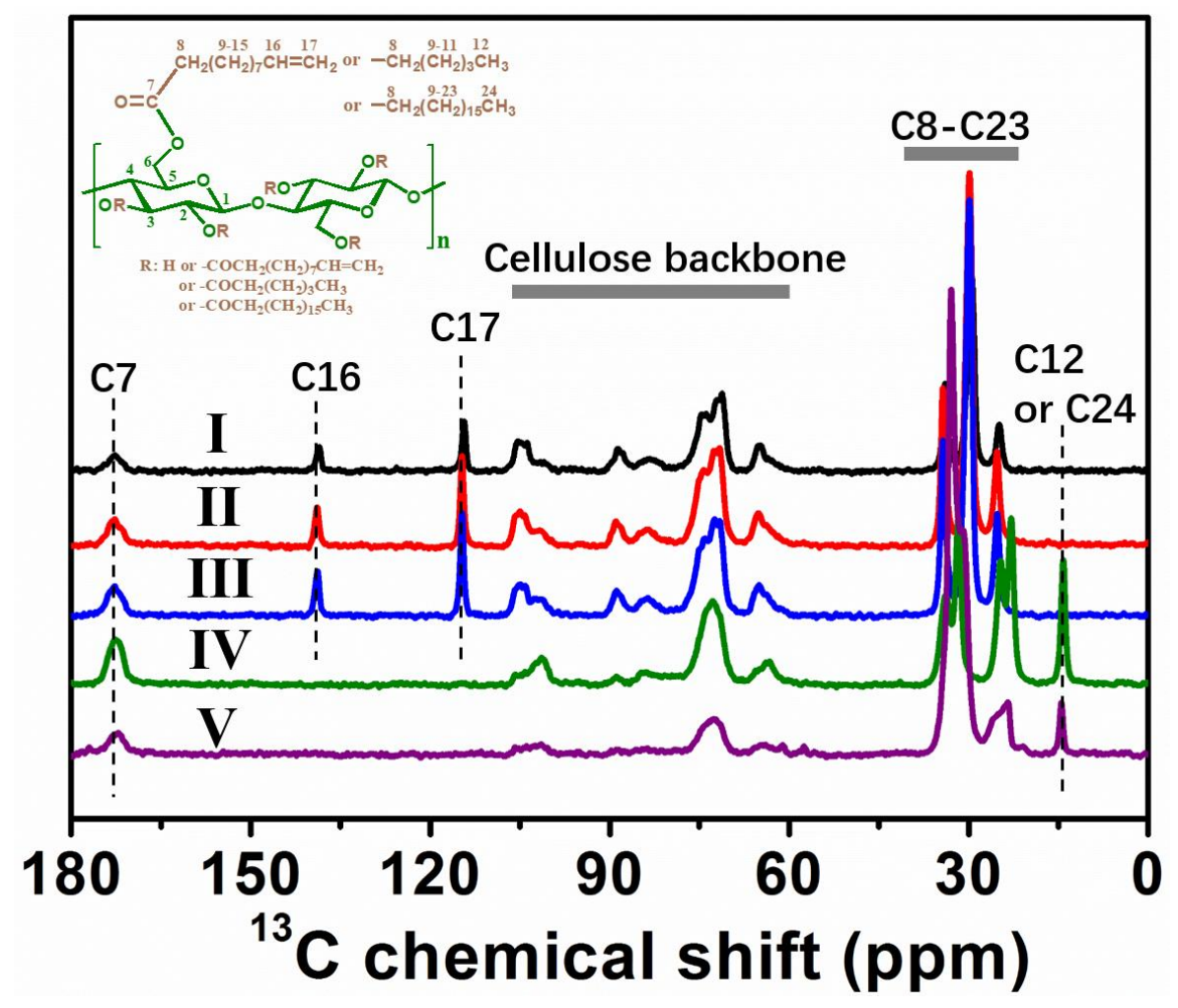

Figure S2. Solid-state CP-MAS ${ }^{13} \mathrm{C}$ NMR spectra of (I) $\mathrm{CNWs}-\mathrm{SU}_{0.98}$, (II) $\mathrm{CNWs}_{-} \mathrm{SU}_{1.15}$, (III) $\mathrm{CNWs}-\mathrm{SU}_{1.66}$, (IV) $\mathrm{CNWs} \mathrm{SH} \mathrm{H}_{1.57}$, (V) CNWs-SS 1.59 .

The solid-state CP-MAS ${ }^{13} \mathrm{C}$ NMR spectrums show the signals assigned to the cellulose backbones, 10-undecenoyl, hexanoyl and stearoyl groups. The signals between 55 and $110 \mathrm{ppm}$ are ascribed to the carbons of anhydroglucose units of cellulose. The chemical shift at $172.6 \mathrm{ppm}$ represents the carbon from the ester linkage. The signals at 114 and $139 \mathrm{ppm}$ attribute to carbons in terminal olefin groups for (I) CNWs-SU ${ }_{0.98}$, (II) $\mathrm{CNWs}_{-} \mathrm{SU}_{1.15}$, and (III) CNWs-SU $\mathrm{CH}_{1.66}$. The signals between 40 and $10 \mathrm{ppm}$ represent the remaining carbon atoms of the 10-undecenoyl, hexanoyl or stearoyl moieties.

a

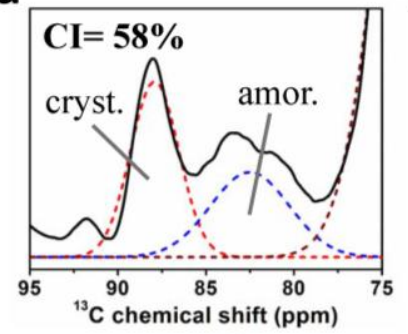

e b

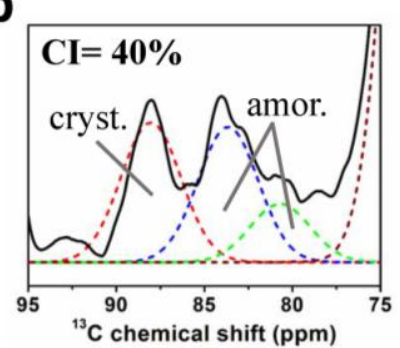

C

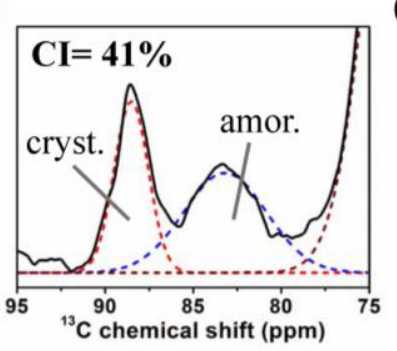

d

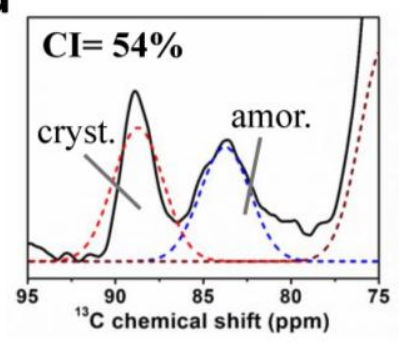

g
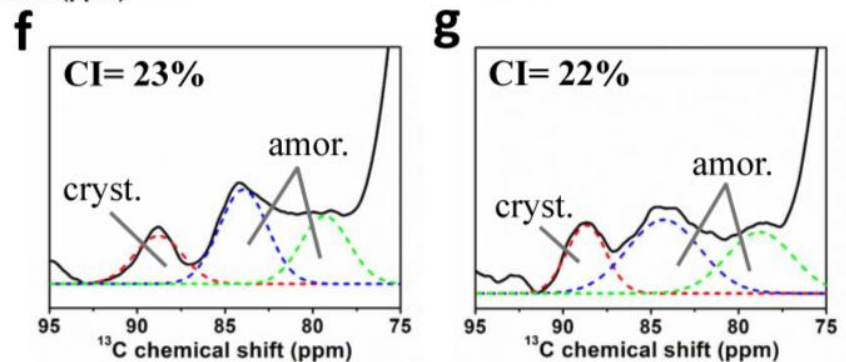

Figure S3. Peak deconvolution for the $\mathrm{C} 4$ region of solid-state CP-MAS ${ }^{13} \mathrm{C}$ NMR spectra of (a) microcrystalline cellulose, (b) CNWs$\mathrm{SU}_{1.32}$, (c) CNWs-SU 0.98 , (d) CNWs-SU ${ }_{1.15}$, (e) CNWs-SU $\mathrm{CN}_{1.66}$, (f) CNWs-SH $\mathrm{CN}_{1.57}$, (g) CNWs-SS $\mathrm{CN}_{1.59}$. 


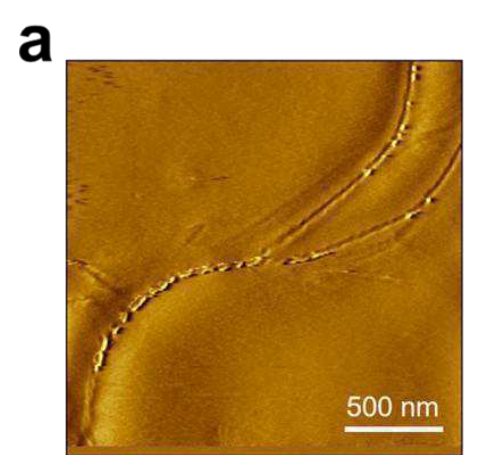

b

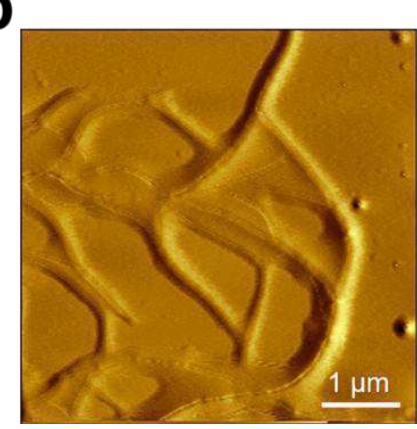

C

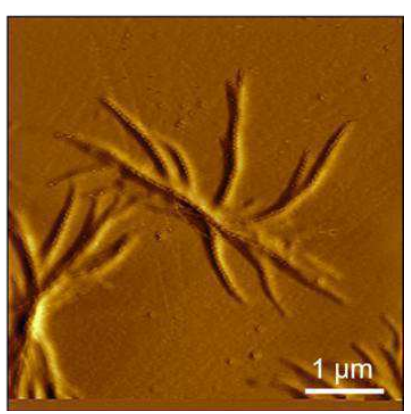

Figure S4. AFM images of (a) PCUE 1.1 , (b) $\mathrm{CUE}_{3}$, (c) $\mathrm{CSE}_{3}$.

a

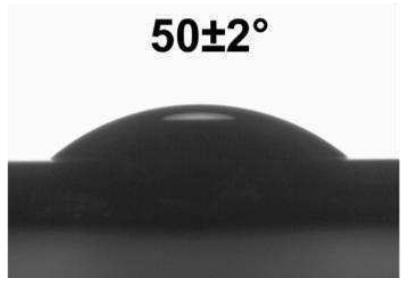

b

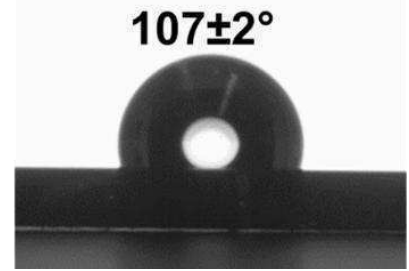

C

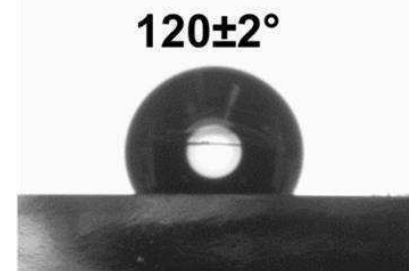

Figure S5. Static water contact angles on the corresponding surfaces. (a) Silicon wafer, (b) silanized silicon wafers with surfaceattached (dimethyl)octadecylsilyl groups, (c) PDMS surface.
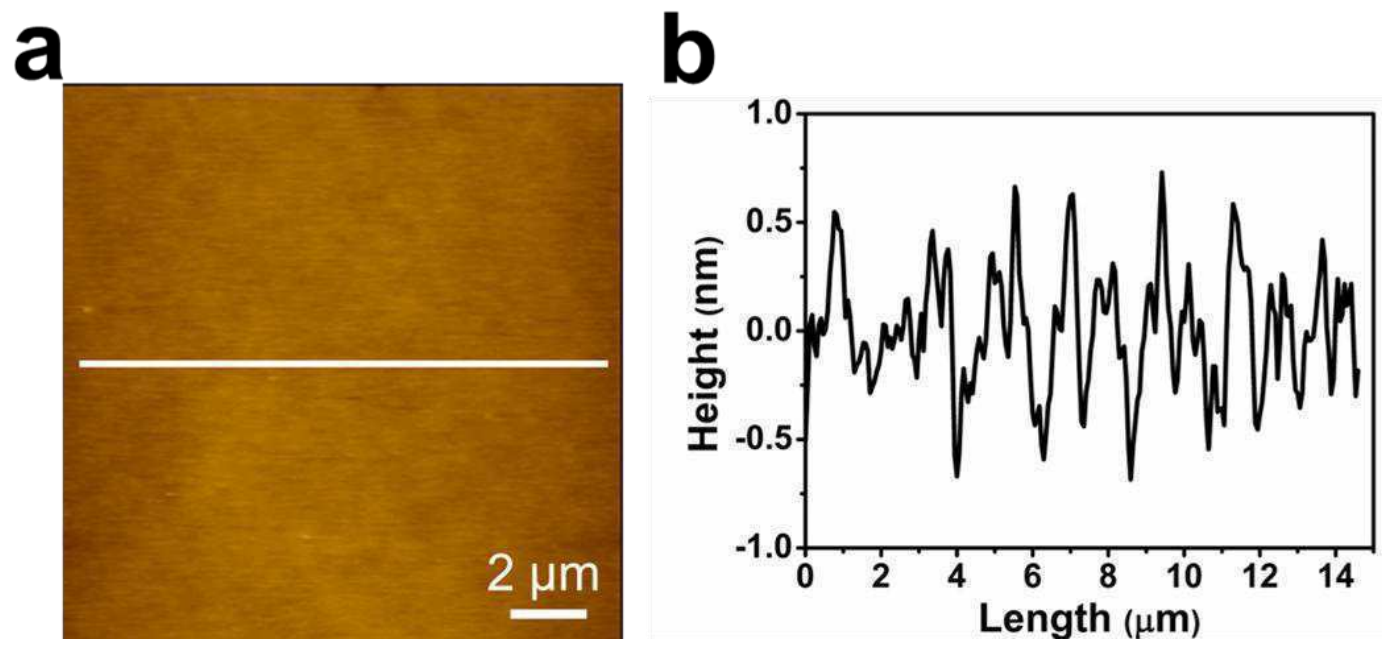

Figure S6. (a) AFM image and (b) corresponding height profile of PDMS surface as shown by the line in (a). 

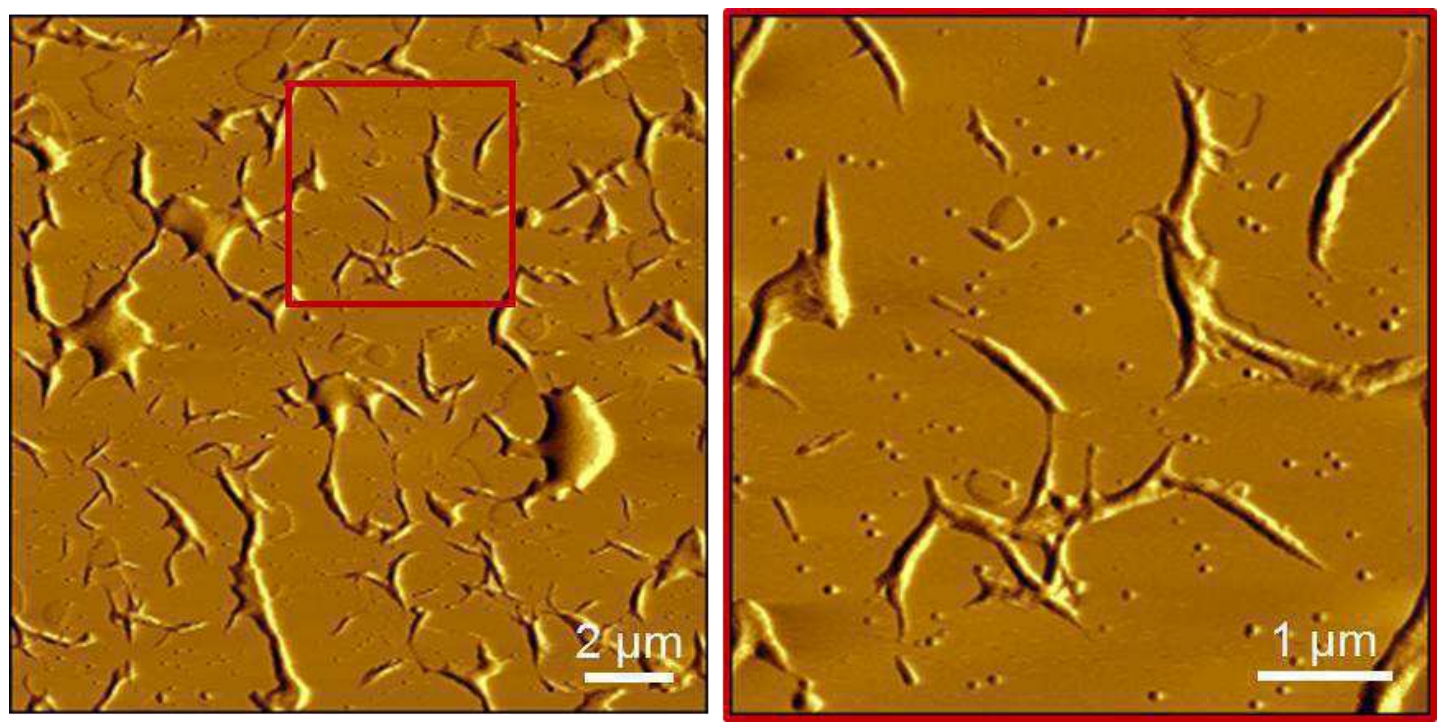

Figure S7. AFM images of dried CNWs-SU ${ }_{1.32}$ on the surface of hydrophobized silicon wafer using their THF suspensions at a concentration of $0.0002 \mathrm{mg} / \mathrm{mL}$ with two different magnifications.
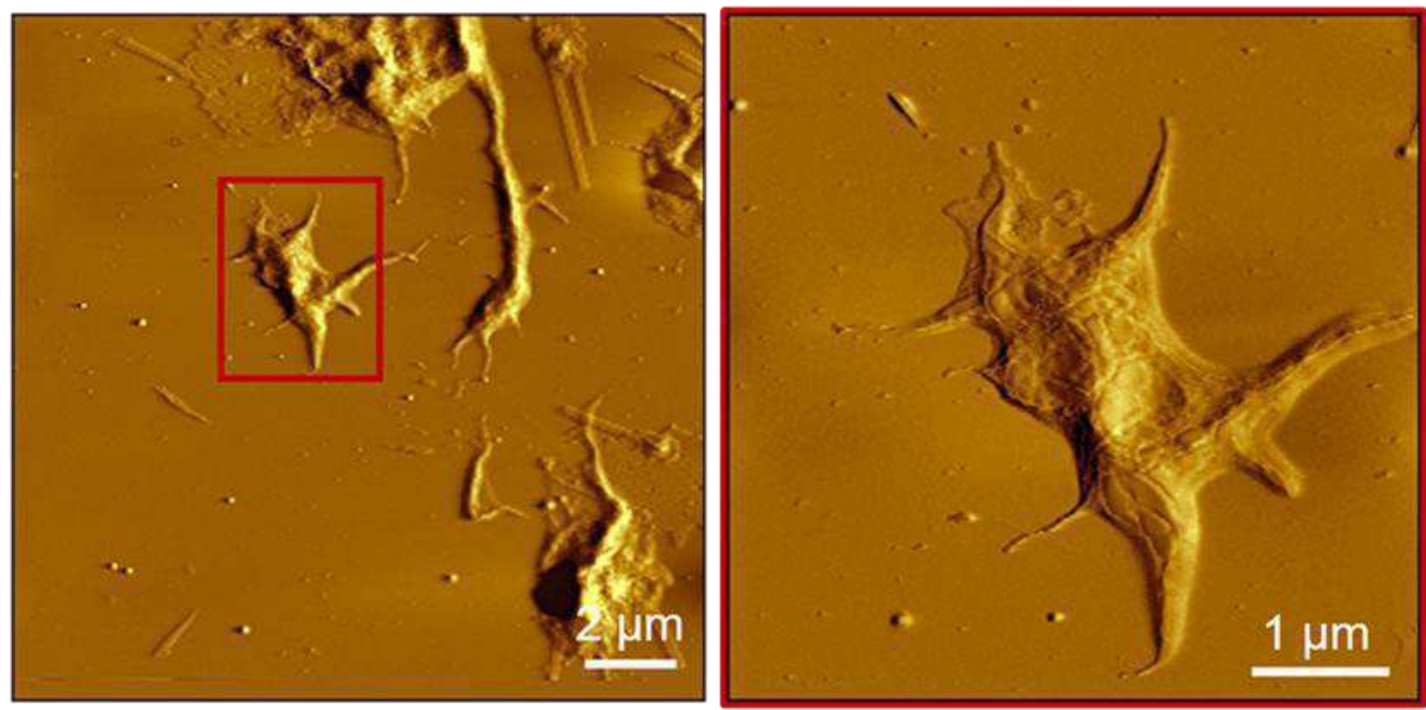

Figure S8. AFM images of dried $\mathrm{CNWs}-\mathrm{SU}_{1.32}$ on silicon wafer using their toluene suspensions at a concentration of $0.0002 \mathrm{mg} / \mathrm{mL}$ with two different magnifications. 

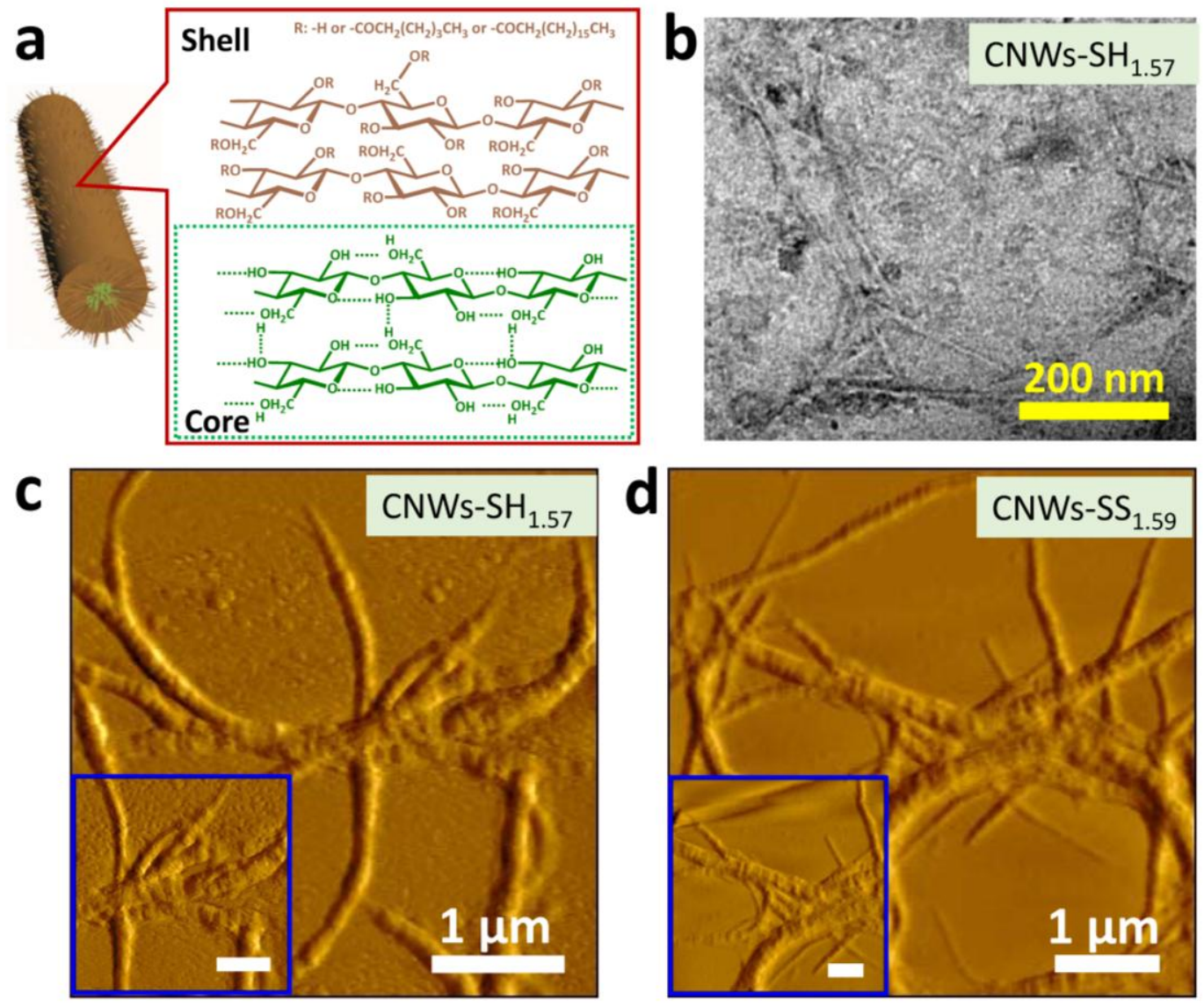

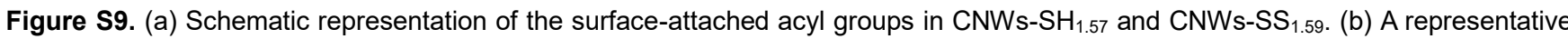
TEM image of $\mathrm{CNWs}-\mathrm{SH}_{1.57}$. (c) AFM amplitude image of $\mathrm{CNWs}^{-\mathrm{SH}_{1.57}}$ dried on silicon wafers using its THF suspension at a concentration of $0.0002 \mathrm{mg} / \mathrm{mL}$. The scale bar in the inset is $500 \mathrm{~nm}$. (d) AFM amplitude image of CNWs-SS 1.59 dried on silicon wafers using its THF suspension at a concentration of $0.0002 \mathrm{mg} / \mathrm{mL}$. The scale bars in the inset are $500 \mathrm{~nm}$.
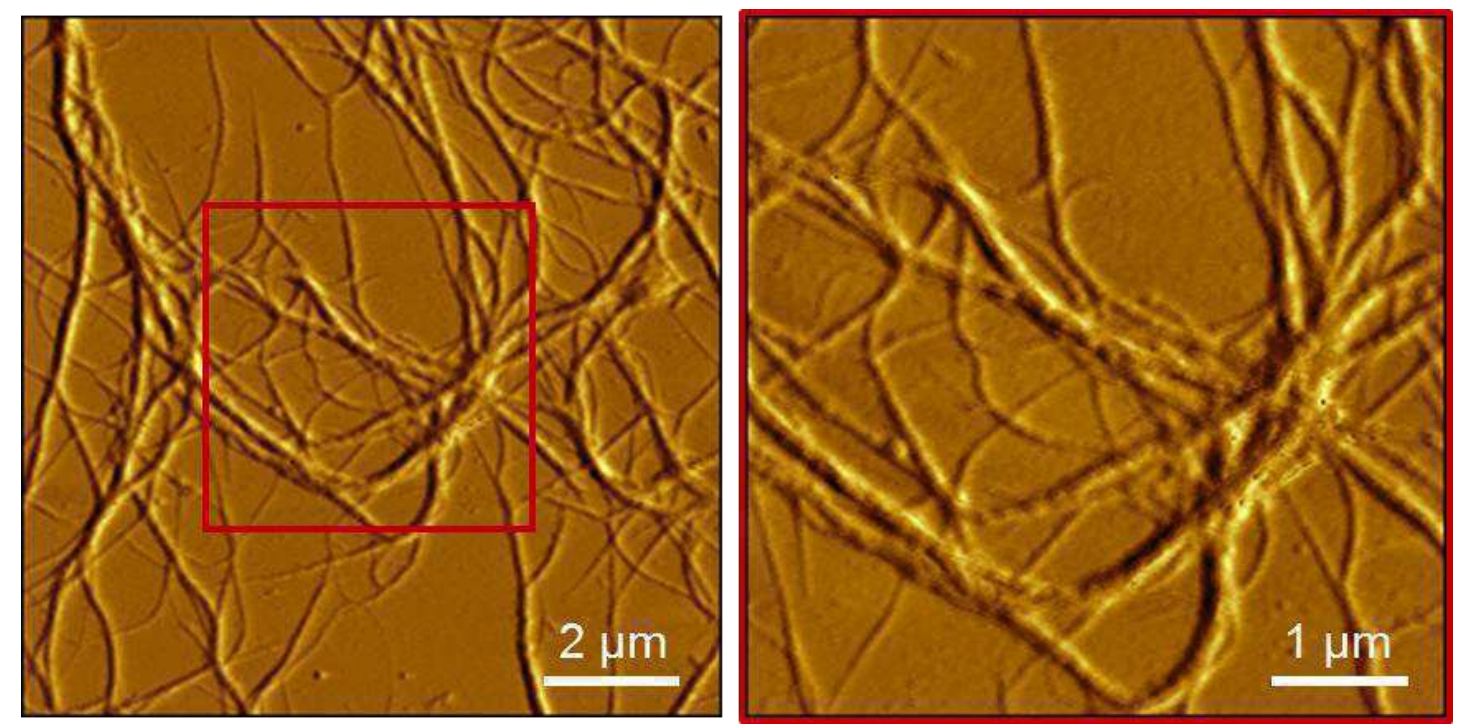

Figure S10. AFM images of dried CNWs-SH 1.37 on silicon wafer using their THF suspensions at a concentration of $0.0002 \mathrm{mg} / \mathrm{mL}$ with two different magnifications. 


\section{References}

[1] Y. Wang, P. B. Groszewicz, S. Rosenfeldt, H. Schmidt, C. A. Volkert, P. Vana, T. Gutmann, G. Buntkowsky, K. Zhang, Adv. Mater. 2017, 29, 1702473.

[2] K. Zhang, A. Geissler, T. Heinze, Part. Part. Syst. 2015, 32, 258-266.

[3] Y. Wang, T. Heinze, K. Zhang, Nanoscale 2016, 8, 648-657.

[4] a) G. Metz, X. L. Wu, S. O. Smith, J. Magn. Reson., Ser. A 110, 219-227; b) A. J. Shaka, J. Keeler, R. Freeman, J. Magn. Reson. (1969) 1983, 53, 313-340.

\section{Author Contributions}

K.Z. conceived the project. H.L. performed synthesis, characterization and self-assembly experiments. B.P. recorded the TEM images. H.L. and R.G. performed and analyzed the AFM measurements. L.A. and R.D. measured NMR spectra. H.L., L.C. and K.Z. discussed and proposed the mechanism. H.L. and K.Z. analyzed the results and all authors contributed to the discussion of the results. H.L. and K.Z. drafted the manuscript and all authors contributed to the writing of the manuscript. 


\section{Publication 2}

Self-assembly of surface-acylated cellulose nanowhiskers and graphene oxide for multiresponsive Janus films with time-dependent dry-state structures

Huan Liu, Bo Pang, Qiyun Tang, Marcus Müller, Hua Zhang, Riza Dervişoğlu, and Kai Zhang*

The article was published online in Small in October 2020. The supplementary materials are attached to the main part. The full article can be found online at:

https://onlinelibrary.wiley.com/doi/full/10.1002/smll.202004922

DOI: $\underline{10.1002 / \mathrm{smll} .202004922}$ 


\title{
Self-Assembly of Surface-Acylated Cellulose Nanowhiskers and Graphene Oxide for Multiresponsive Janus-Like Films with Time-Dependent Dry-State Structures
}

\author{
Huan Liu, Bo Pang, Qiyun Tang,* Marcus Müller, Hua Zhang, Riza Dervişoğlu, \\ and Kai Zhang**
}

For the first time Janus-like films of surface-acylated cellulose nanowhiskers (CNWs) with or without graphene oxide (GO) via one-step evaporationdriven self-assembly process are reported, which have reconstructible time-dependent micro-/nanostructures and asymmetric wettability. The heterogeneous aggregation of CNWs on rough Teflon substrates favors the formation of uniform films, leading to hydrophobic smooth bottom surface. The homogeneous nucleation of residual CNWs in bulk suspensions promotes the growth of patchy microspheres with an average diameter of $22.7 \pm 2.1 \mu \mathrm{m}$, which precipitate on the top surface leading to enhanced hydrophobicity. These patchy microspheres are thermoresponsive and vanish after heating at $60^{\circ} \mathrm{C}$ within $1 \mathrm{~min}$, while they are reconstructed at room temperature with time-dependent evolving micro-/nanostructures in dry state within $2 \mathrm{~d}$. The thermoresponsive transition of patchy microparticles leads to accompanied switchable change between transparency and opacity of Janus-like films. Furthermore, the incorporation of $\mathrm{GO}$ generates more patchy microspheres with an average diameter of $13.5 \pm 1.3 \mu \mathrm{m}$ on the top surface of hybrid Janus-like films. Different distributions of CNWs and GO in Janus-like films and the solvent-responsive self-assembled patchy microparticles of CNWs facilitate their reversible actuation by showing fast curling in THF within $6 \mathrm{~s}$ and flattening in water for at least 25 cycles.
H. Liu, B. Pang, H. Zhang, Prof. K. Zhang

Department Wood Technology and Wood-Based Composites

University of Goettingen

Büsgenweg 4, Göttingen 37077, Germany

E-mail: kai.zhang@uni-goettingen.de

Dr. Q. Tang, Prof. M. Müller

Institute for Theoretical Physics

University of Goettingen

Friedrich-Hund-Platz 1, Göttingen 37077, Germany

E-mail: qiyun.tang@theorie.physik.uni-goettingen.de

Dr. R. Dervişoğlu

NMR-Based Structural Biology

Max-Planck-Institute for Biophysical Chemistry

Am Fassberg 11, Göttingen 37077, Germany

The ORCID identification number(s) for the author(s) of this article can be found under https://doi.org/10.1002/smll.202004922.

(c) 2020 The Authors. Published by Wiley-VCH GmbH. This is an open access article under the terms of the Creative Commons AttributionNonCommercial License, which permits use, distribution and reproduction in any medium, provided the original work is properly cited and is not used for commercial purposes.

\section{Introduction}

Self-assembly of nanoparticles into ordered materials is an attractive route for the synthesis of functional materials that are not feasible through conventional techniques. ${ }^{[1]}$ Macroscopic assemblies of nanoscaled materials have the potential to revolutionize a wide variety of technologies, including photovoltaics, plasmonics, magnetic storage, and sensing. ${ }^{[2]}$ In general, the self-assembly process strongly depends on the properties of the nanoscale building blocks and the macroscopic parameters that characterize the assembly process. Recently, much attention has been paid to self-assembly of anisotropic cellulose nanowhiskers (CNWs) because of their unique properties, such as high surface to volume ratio, high mechanical performance, small thermal expansion coefficient, renewability, and sustainability. ${ }^{[3]}$ This self-assembly phenomenon of CNWs was shown by the occurrence of "fingerprint" patterns obtained from suspensions observed by polarized light microscopy (PLM), indicating chiral-nematic ordering. This chiral nematic structure can even be preserved within the iridescent films that are obtained after the evaporation of CNWs suspensions on a flat substrate. ${ }^{[4]}$ Many factors during the self-assembly influence the optical and mechanical properties of obtained films, including the temperature, pressure, substrate and surface chemistry of the CNWs. ${ }^{[5]}$ For instance, we recently reported the formation of helical fibers with lengths of micrometers by the evaporation-driven self-assembly on silicon wafers of functionalized CNWs with surface-attached acyl chains. ${ }^{[6]}$

Although the self-assembly of CNWs and their potential applications for the fabrication of nanocomposites and interaction with other nanoparticles have been extensively studied, the most commonly used dispersion media are aqueous solutions, which restricts the interaction of homogeneously dispersed CNWs and hydrophobic polymer matrices in nonpolar solvents. ${ }^{[7]}$ This is mainly because CNWs from native cellulose fibers are generally decorated with polar moieties on surface and thus are basically hydrophilic in nature. CNWs readily

DOI: 10.1002/smll.202004922 
aggregate in organic solvents or in the presence of hydrophobic polymers, without forming continuous materials with sufficient mechanical stabilities. Thus, surface modifications are often required before suspending in an organic solvent or compounding with hydrophobic substances. ${ }^{[8]}$ Moreover, nanoparticles with different shapes, dimensions, and charges would also affect the organization of CNWs. Coassembly of nanoparticles with the distinct shape, size-, and compositiondependent properties is a promising approach for the preparation of multicomponent nanostructured materials with new or enhanced properties. ${ }^{[9]}$ Graphene oxide (GO) as a kind of 2D nanomaterial has been widely used to combine with hydrophilic nanocellulose to prepare functional materials owing to its outstanding mechanical properties, light weight, high surface area, potential electrical conductivity, and aqueous processability. ${ }^{[10]}$ While most studies mainly focused on improving the mechanical or electronic properties of obtained materials, to the best of our knowledge, using GO as a polar nanomaterial to tune the self-assembly process of 1D nonpolar CNWs has not been explored.

Furthermore, Janus films as a class of functional materials possess asymmetric architecture based on two incompatible sides of different chemistry or morphology. In general, the Janus films can be fabricated by two approaches as asymmetric fabrication and asymmetric decoration. However, the complete manufacture of Janus films still needs a couple of steps. In recent years, major progress has been achieved for the preparation of nano/microsized Janus materials/particles by self-assembly, modification with mask protection or phase separation. ${ }^{[1]}$ Self-assembly is among them an elegant and powerful way to fabricate materials with novel structure and properties, which is also an essential feature of many strategies to generate Janus structures on nano/microscale. However, still very few reports focus on the fabrication of Janus films via self-assembly until now, let alone the Janus films using nanocellulose via self-assembly. Zhang et al. fabricated flexible Janus films with integrated electrically, optically, and thermally anisotropic properties by simply mixing liquid metal nanoparticles with suspensions of CNWs and polyvinyl alcohol, followed by self-assembly density deposition. ${ }^{[12]}$

Here, we report a facile self-assembly process of CNWsSU-C18, which were obtained after the UV-induced thiol-ene reaction of CNWs with surface-attached 10-undecenoyl moieties (CNWs-SU) with 1-octadecanethiol, for the preparation of multiresponsive Janus-like films. These films had asymmetric topography on their top and bottom surfaces with different wettabilities. Furthermore, these self-assembled structures vanished after heating and recovered after cooling down to room temperature over time. Moreover, the self-assembly process forming such surface structures was altered by incorporating GO into the matrix of CNWs-SU-C18 due to their different wettabilities, which promoted the phase separation during the drying process. Resulting Janus-like films were thermoresponsive and represented excellent substrates for solvent-responsive actuators. Thus, our method demonstrates a facile strategy for the one-step preparation of multiresponsive Janus-like films via the self-assembly of CNWs-SU-C18 solely or as composites with GO.

\section{Results and Discussion}

\subsection{Synthesis of CNWs-SU and CNWs-SU-C18}

CNWs with surface-immobilized 10-undecenoyl groups (CNWs$\mathrm{SU})$ were synthesized with a yield of about $10 \%$ from microcrystalline cellulose (MCC) after the surface esterification by 10-undecenoyl chloride (Figure 1a). The degree of substitution (DS) of CNWs-SU was measured to be 1.32 and the content of surface-immobilized 10-undecenoyl groups was determined to be about $3.57 \mathrm{mmol} \mathrm{g}^{-1}$ based on the elemental analysis. TEM image (Figure $1 \mathrm{~b}$ ) displays that CNWs-SU had typical whisker shapes with an average length of $135 \pm 37 \mathrm{~nm}$ and an average diameter of $4 \pm 1 \mathrm{~nm}$ based on the measurement of 100 single CNWs (Figure S1, Supporting Information). The solid-state ${ }^{13} \mathrm{C}$ CP-MAS NMR spectra showed characteristic bands ascribed to both cellulose backbone and 10-undecenoyl groups (Figure 1d). The signals between 55 and $110 \mathrm{ppm}$ are assigned mainly to the carbons of anhydroglucose units of cellulose. The signals between 40 and $10 \mathrm{ppm}$ are attributed to the carbons of aliphatic groups and the peak at $173 \mathrm{ppm}$ is ascribed to the carbonyl carbon from the ester linkages. The signals at 114 and $139 \mathrm{ppm}$ are attributed to carbons in terminal olefin groups. Based on the signals ascribed to crystalline $(88.5 \mathrm{ppm})$ and amorphous $(83.7 \mathrm{ppm})$ domains, ${ }^{[13]}$ the crystallinities of MCC and CNWs-SU are determined to be $\approx 58 \%$ and $40 \%$, respectively (Figure S2a,b, Supporting Information).

CNWs-SU-C18 was obtained after the thiol-ene reaction of the terminal olefin groups with 1-octadecanethiol. Three molar ratios of thiols to $\mathrm{C}=\mathrm{C}$ double bonds of $100 / 100,75 / 100$, and 25/100 were used, namely CNWs-SU-C18/100, CNWs-SUC18/75, and CNWs-SU-C18/25, respectively. Compared with the ${ }^{13} \mathrm{C}$ CP-MAS NMR spectra of CNWs-SU, a new signal emerged at $15 \mathrm{ppm}(\mathrm{C} 35)$ and is ascribed to the terminal methyl carbons of the long-chain hydrocarbons, which suggests the successful introduction of thiols via the thiol-ene reaction for CNWS-SUC18 (Figure 1d). ${ }^{[14]}$ The crystallinity of CNWs-SU-C18/75 was determined to be about $33 \%$ according to the ${ }^{13} \mathrm{C}$ CP-MAS NMR spectra (Figure S2c, Supporting Information). Based on the elemental analysis, the contents of 1-octadecane groups in CNWs-SU-C18/100, CNWs-SU-C18/75, and CNWs-SU$\mathrm{C} 18 / 25$, were determined to be $1.55,1.37$, and $0.55 \mathrm{mmol} \mathrm{g}^{-1}$, respectively. Compared with CNWs-SU, CNWs-SU-C18 still maintained their original sizes after the introduction of further alkane moieties. For instance, CNWs-SU-C18/75 had an average dimension of $146 \pm 33 \mathrm{~nm}$ in length and $4 \pm 1 \mathrm{~nm}$ in diameter (Figure 1c).

\subsection{Fabrication of Self-Standing Films Using CNWs-SU-C18}

Solvent-casting THF suspensions of CNWs-SU-C18/25, CNWsSU-C18/75, or CNWs-SU-C18/100 on Teflon substrates resulted in self-standing films (Figure 2a and Figure S3, Supporting Information). Comparing with CNWs-SU-C18/75 films, films of CNWs-SU-C18/25 and CNWs-SU-C18/100 had relatively lower homogeneity and even cracks.

Moreover, the top surface of as-prepared CNWs-SU-C18/75 films contained hierarchical micro/nanostructure. Many 

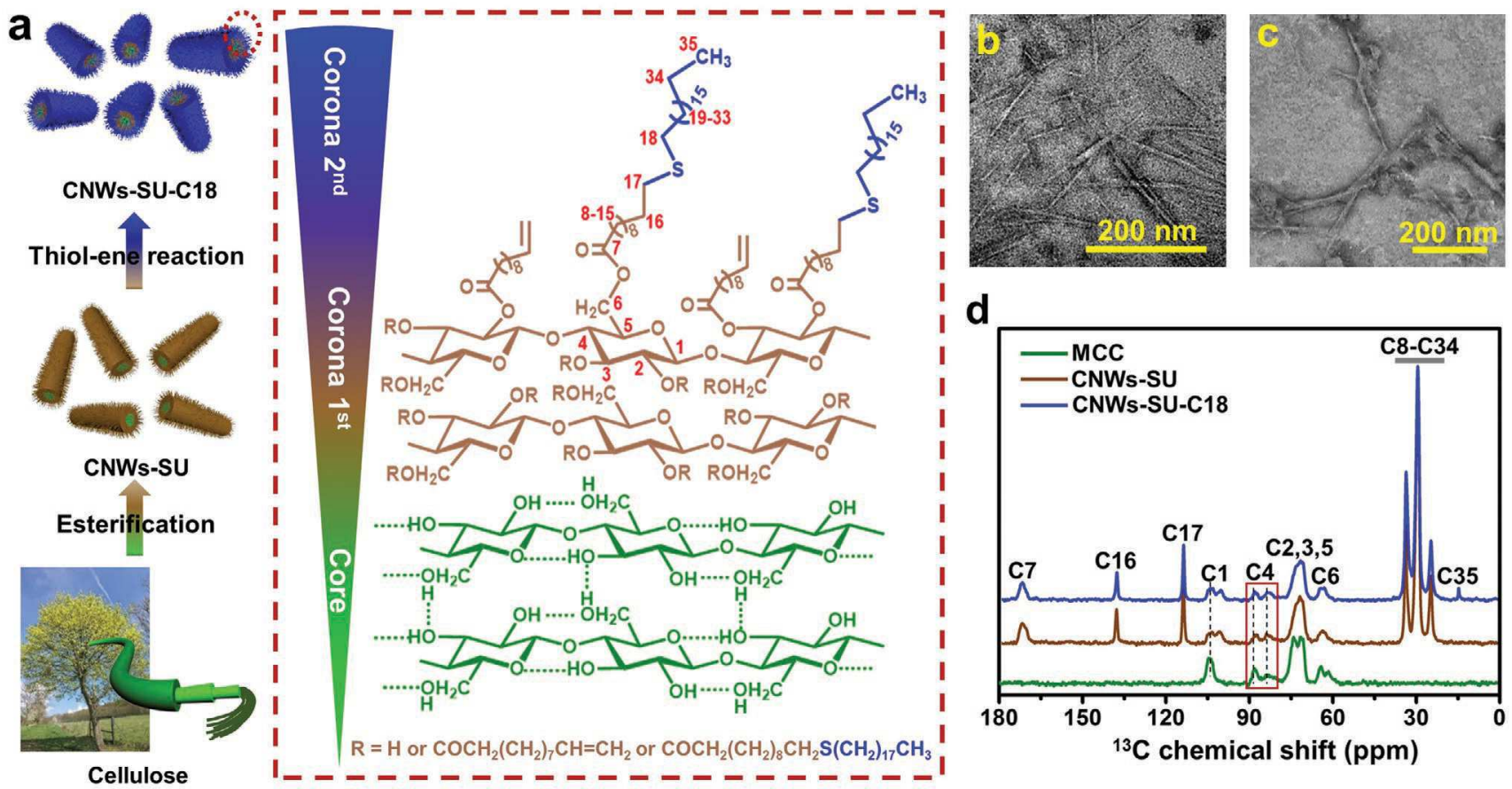

Figure 1. Synthesis and characterization of surface-acylated CNWs. a) Schematic illustration for the synthesis of CNWs-SU and CNWs-SU-C18. b) A representative TEM image of CNWs-SU. c) A representative TEM image of CNWs-SU-C18. d) Solid-state ${ }^{13}$ C CP-MAS NMR spectra of microcrystalline cellulose, CNWs-SU and CNWs-SU-C18.

spherical microscale particles with an average diameter of $22.7 \pm 2.1 \mu \mathrm{m}$ were randomly distributed on the top surface of CNWs-SU-C18/75 films (Figure 2b). Figure 2c shows a highresolution SEM image of a single particle, where lamellar nanoplates with an average thickness of $196 \pm 36 \mathrm{~nm}$ are vertically present on the whole surface of microparticles. These selfassembled hierarchical structures on the top surface led to highly hydrophobic surfaces with the static water contact angle (SWCA) of $125 \pm 2^{\circ}$. In comparison, the SWCA of the smooth bottom surface was only $95 \pm 1^{\circ}$ (Figure S4, Supporting Information). The high stable hydrophobicity on the top surface of CNWs-SUC18/75 films should be attributed to the present hierarchical micro/nanostructures, as well known for lotus leaves with combined micropapillary and nanobranches. ${ }^{[15]}$ Moreover, these spherical microscale particles showed multiple colors under the PLM, indicating the presence of ordered domains within these self-assembled structures (Figure S5, Supporting Information).

Furthermore, the nontransparent CNWs-SU-C18/75 films turned transparent after the treatment at $60{ }^{\circ} \mathrm{C}$ within $1 \mathrm{~min}$ due to the disappearance of microparticles (Figure $2 \mathrm{~d}$ and Movie S1, Supporting Information). $60^{\circ} \mathrm{C}$ is the lowest temperature for turning nontransparent CNWs-SU-C18/75 films into transparent during heating. When the temperature is higher than $60{ }^{\circ} \mathrm{C}$, such as $70{ }^{\circ} \mathrm{C}$ or $80^{\circ} \mathrm{C}$, the films can also turn transparent in a similar manner as the treatment at $60^{\circ} \mathrm{C}$. However, when the temperature is lower than $60{ }^{\circ} \mathrm{C}$, the films cannot become transparent and only keep their original state. When the temperature was lowered to room temperature, the films became non-transparent again due to the formation of microparticles. The cooling down from $60{ }^{\circ} \mathrm{C}$ to room temperature took less than $2 \mathrm{~min}$, while it took a few further days for the films to become non-transparent again. During the cooling process, the lamellar nanoplates on spherical microparticles were gradually reformed (Figure 2e). The change of the morphology and sizes of microparticles will be discussed later in Figure 3. The wettability of the recovered top surface maintained almost the same with the SWCA of about $123 \pm 2^{\circ}$. The diameter of these flat particles was averagely $21.3 \pm 1.2 \mu \mathrm{m}$. The thickness of these nanoplates lied at $195 \pm 40 \mathrm{~nm}$ (Figure 2f), which was similar to the original size in as-prepared spheres (Figure 2c).

In comparison, the films of CNWs-SU were only transparent films with smooth surfaces and did not contain such self-assembled structures at all (Figure S6, Supporting Information). Thus, the octadecane chains at the surface in the outmost corona of CNWs-SU plays a key role during the self-assembly in the dry state.

Two mechanisms contribute to the formation of Janus-like films via solvent evaporation. Initially, CNWs-SU-C18 rods are dispersed in suspensions at a low concentration. On the one hand, as the solvents gradually evaporate, the increase of rod concentrations promotes the nucleation at high concentrations. Here, the Teflon substrates have a surface roughness of around $437 \mathrm{~nm}$ (see Figure S7, Supporting Information). The randomly distributed domains on the substrates can be considered as the heterogeneous nuclei, which facilitate the nucleation of rods on the Teflon substrates at sufficiently high concentrations of rods (Figure 2g). Therefore, the formation of CNWs-SU-C18 films presumably proceeds with the heterogeneous nucleation of rods on the rough Teflon substrates.

On the other hand, the residual rods in the bulk suspension still increase their concentration during the solvent evaporation, which decreases the nucleation barrier of the 

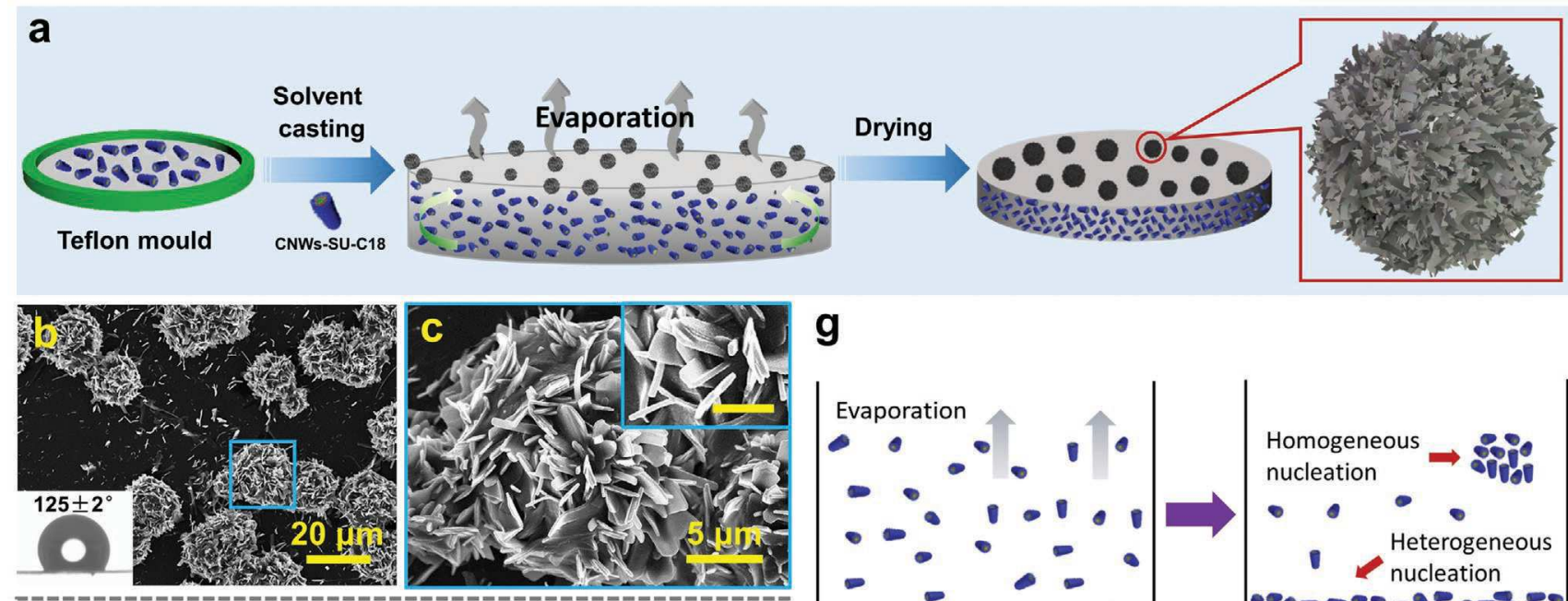

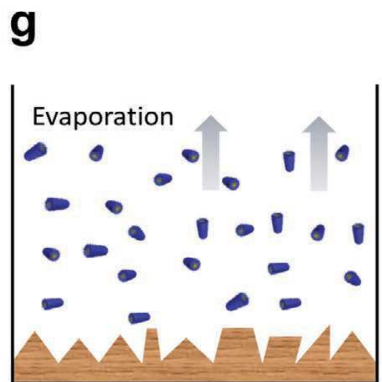

Teflon substrates

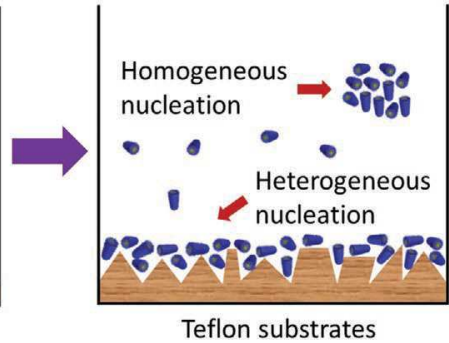

Teflon substrates

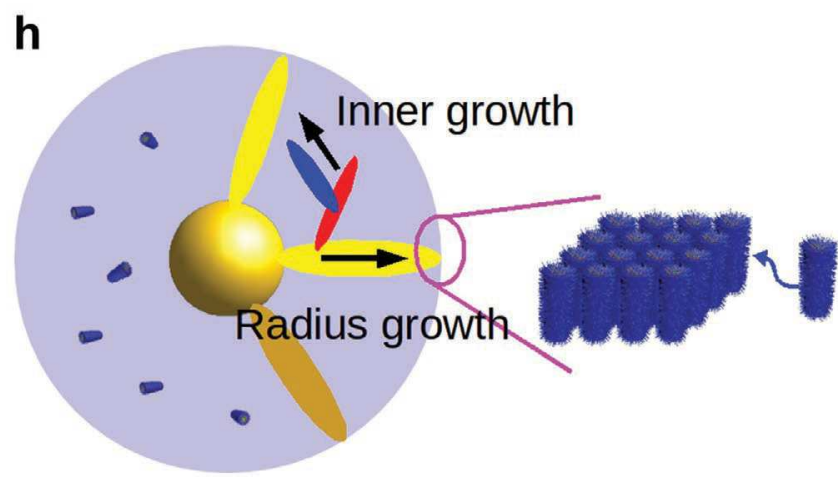

Figure 2. Preparation and characterization of CNWs-SU-C18 films. a) Schematic representation for the fabrication of CNWs-SU-C18 films by solventcasting. b) Representative SEM image of the top surface of the CNWs-SU-C18/75 film. The inset shows static water contact angle (SWCA) on the top surface. c) SEM image of the magnified region as in (b). The inset shows the magnified particle surface in (c) with the scale bar of $2 \mu \mathrm{m}$. d) Photo images of one CNWs-SU-C18/75 film on flowers at $25^{\circ} \mathrm{C}$ and $60^{\circ} \mathrm{C}$. e) Representative SEM image of the CNWs-SU-C18/75 film after heat-treatment at $60^{\circ} \mathrm{C}$, cooling down to room temperature and further aging at room temperature for more than two days. The inset shows SWCA on this surface. f) SEM image of the magnified region as in (e). The inset shows the magnified particle surface in (f) with the scale bar of $2 \mu \mathrm{m}$. g) Illustration of two mechanisms governing the formation of Janus-like CNWs-SU-C18 films during the solvent evaporation. The rough surface of Teflon substrates provides randomly distributed nuclei that promote the heterogeneous nucleation of rods, forming CNWs-SU-C18 thin films. The residual rods in suspension form clusters via homogeneous nucleation as the rod concentration increases during the solvent evaporation. $h$ ) Illustration of the growth of patchy microparticles. The growth of yellow sheets increases the radius of particles, whereas the growth of red or blue disks from the existing sheets fills up the particles. The attachment of CNWs-SU-C18 to the cluster gives rise to the growth of the sheets.

CNWs-SU-C18 in suspension and facilitates their aggregation to clusters (Figure $2 \mathrm{~g}$ ). The octadecane alkane chains on the rods tend to attach to the clusters, driving the rod to be parallel to each other and forming lamellar sheets (Figure 2h). This is similar to polymer crystallization ${ }^{[16]}$ in the sense that rods are attracted to be parallel to each other at suitable conditions. Such process indicates a typical homogeneous nucleation and growth process. Further growth of the sheets at their edge and nucleation of new sheets on existing ones result in the formation of patchy microparticles, which are similar to the structures observed in other crystal systems. ${ }^{[17]}$ The growth of patchy microparticles could be separated into two processes: i) the attachment of rods to the outer edges of crystalline sheets increases the radius of patchy microparticles, ii) the nucleation and growth of crystalline sheets on the existing ones presumably result in the inner growth of patchy microparticles, which tends to fill up the structure, as shown in Figure $2 \mathrm{~h}$. As the radius increases, the inner growth consumes a large fraction of CNWs-SU-C18 that diffuses toward the patchy microparticles from bulk suspensions, which slows down the radial growth of patchy microparticles. Generally speaking, homogeneous nucleation is a rare event, giving rise to widely size-distributed nuclei. However, the increase of concentration driven by evaporation will facilitate the nucleation of CNWs-SU-C18, making the time distribution of nucleation events narrower than that at fixed concentration. This effect as well as the slow growth of patchy microparticles give rise to the narrow size distribution of microspheres, as shown in Figure 2b. 

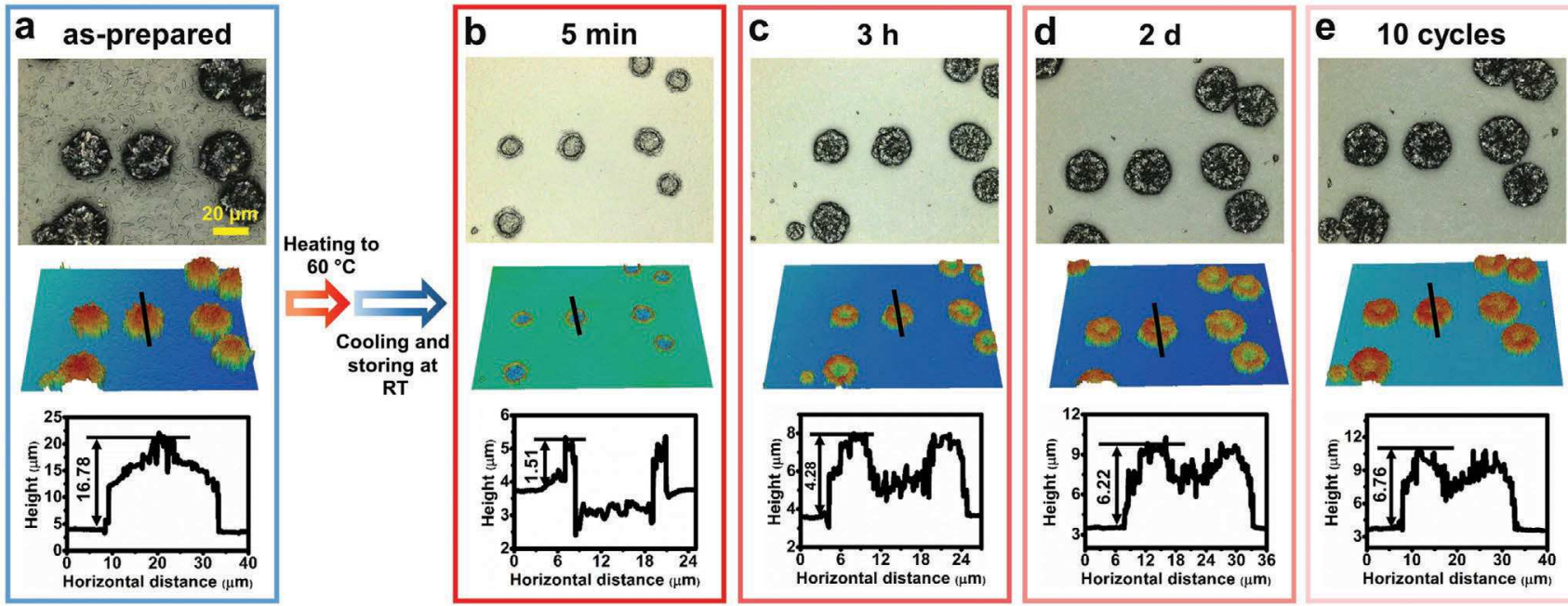

Figure 3. Thermoreversible properties of CNWs-SU-C18/75 film. a) Representative laser scanning microscope (LSM) image, 3D LSM image and height profile of microparticles on the top surface of a CNWs-SU-C18/75 film. b-d) Time-dependent LSM images, 3D LSM images, and height profiles of the self-assembled structures after cooling down from $60^{\circ} \mathrm{C}$ to room temperature and further aging time of $5 \mathrm{~min}, 3 \mathrm{~h}$, and $2 \mathrm{~d}$, respectively. e) LSM image, 3D LSM image, and height profile of the CNWs-SU-C18/75 film after 10 cycles of heating-cooling process. The images and height profile after the $10^{\text {th }}$ cycle were recorded after maintaining at room temperature for $2 \mathrm{~d}$. RT: room temperature.

Remarkably, the laser scanning microscope (LSM) measurement also reveals that these self-assembled structures exhibited thermo-reversible properties (Figure 3). Microsized spherical particles on the top surface of as-prepared CNWs-SU-C18/75 films were also clearly recorded using LSM, which showed similar morphology as in their SEM images (Figures 2b,c and $3 \mathrm{a})$. According to the height profiles obtained from the 3D LSM image, the height of the initial microparticles was about $14.2 \pm 1.0 \mu \mathrm{m}$ and the diameter was averagely $24.9 \pm 1.8 \mu \mathrm{m}$.

Immediately after cooling down from heat-treatment at $60^{\circ} \mathrm{C}$ and maintaining at room temperature for $5 \mathrm{~min}$, circled structures were formed and grew gradually, as visualized by 3D LSM images of CNWs-SU-C18/75 films (Figure 3b). The circles have a height of about $1.5 \mu \mathrm{m}$ and a wall thickness of $1.4 \mu \mathrm{m}$. After $3 \mathrm{~h}$ aging at room temperature, a donut-like structure with the diameter of microscale gradually formed due to the expanding of the circles (Figure 3c). After $2 \mathrm{~d}$, the height of the flat donuts further increased to $6.2 \mu \mathrm{m}$ and the wall thickness reached $6.6 \mu \mathrm{m}$ (Figure $3 \mathrm{~d}$ ). The outside diameter was about $22.8 \pm 1.3 \mu \mathrm{m}$. The height of these donuts barely grew after 6 further $d$ and stayed at about $6.42 \mu \mathrm{m}$, while the wall thickness only increased slightly. Besides, the heights and the wall thickness of these donuts remained unchanged after $12 \mathrm{~d}$ of aging at room temperature (Figure S8, Supporting Information).

Although these donut-like microparticles are different from the original spheres in the as-prepared films, they can be reconstructed during the following heating-cooling cycles with constant thermo-reversible properties. No significant fatigue of the morphology was observed after 10 cycles except for the slightly variable outside diameters of the donuts (Figure 3e). The regeneration of such self-assembled donut-like particles was assumed to be realized via two steps: melting and recrystallization. It is worth noting that the melting happens on the crystal structures formed with octadecane alkane chains and not on the crystalline part of CNWs. The observation that the outside diameter of the donuts remained unaltered by multiple heating-cooling cycles suggested that the contact line of the molten material was pinned at the surface of Janus-like films. After the first heating, the molten material densified, i.e., the volume of the molten drop was smaller than that of the original sphere. This volume shrinkage in conjunction with the pinned contact line resulted in the formation of a molten rim. After rapid cooling down to room temperature and aging, the molten rim crystallizes, leading to the donut structures. The regeneration of such microsized particles leads to the significant transition of the surface topography, which dominates the recovery of the nontransparency and hydrophobicity of CNWs-SU-C18/75 films.

\subsection{Fabrication of Hybrid Self-Standing Films Using CNWs-SU-C18+GO}

To further adjust the morphology and the surface properties of obtained films, GO with a different surface wettability was added in the suspensions of CNWs-SU-C18/75 to prepare Janus-like films (Figure 4a). GO nanosheets with a smooth surface and a thickness of $\approx 2 \mathrm{~nm}$ were used (Figure S9, Supporting information), ${ }^{[18]}$ according to atomic force microscopy (AFM) analysis. After the one-step preparation via solvent casting, hybrid Janus-like films of CNWs-SU-C18/75 and GO were obtained (Figure 4a and Figure S10, Supporting Information).

Figure $4 \mathrm{~b}$ shows a typical SEM image of the top surface of as-prepared Janus-like films containing $20 \mathrm{wt} \%$ of GO, and these films are referred to as CNWs-SU-C18/75+GO(4:1). Many spherical particles are visible on the top surface in a random pattern with an average diameter of about $13.5 \pm 1.3 \mu \mathrm{m}$. As can be seen from the magnified image of a single particle (Figure 4c), the lamellar nanoplates with an average thickness of $164 \pm 34 \mathrm{~nm}$ protruded radially from the particles forming hierarchical micro- and nanoscaled structures. Following this, 


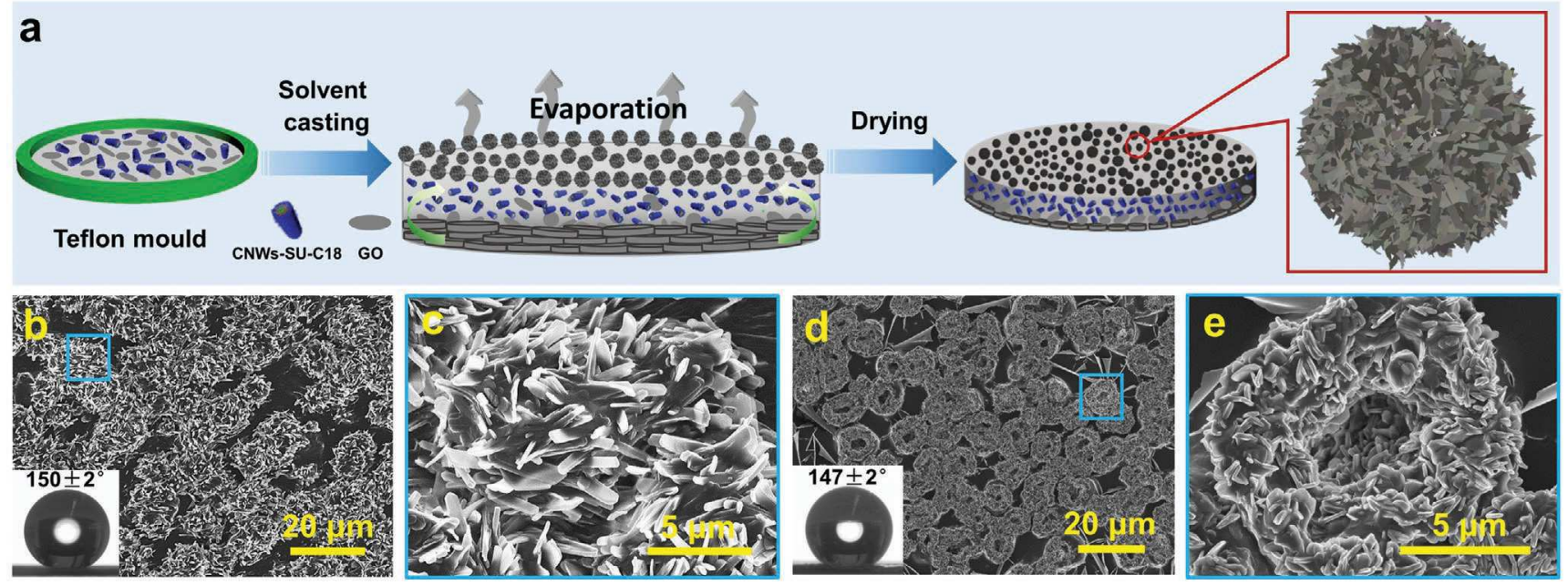

Figure 4. Preparation and characterization of CNWs-SU-C18/75+GO films. a) Schematic representation for the fabrication of CNWs-SU-C18/75+GO films by solvent-casting on Teflon substrates. b) Representative SEM image of the top surface of a CNWs-SU-C18/75+CO(4:1) film. The inset shows SWCA on the top surface. c) SEM image for the magnified region as in (b). d) Representative SEM image of the top surface of CNWs-SU-C18/75+CO(4:1) film after heating at $60^{\circ} \mathrm{C}$, cooling down to room temperature and maintaining at room temperature for more than two days. The inset shows SWCA on the surface. e) SEM image for the magnified region as in (d).

the top surface of these films showed superhydrophobicity with a SWCA of $150 \pm 2^{\circ}$, while the SWCA of the smooth bottom surface was only $96 \pm 3^{\circ}$ (Figure S11a, Supporting Information). Both SEM and the 3D LSM image of the smooth bottom surface of CNWs-SU-C18/75+GO(4:1) films demonstrate the absence of any particular micro- or nanostructures (Figure S11, Supporting Information).

Furthermore, the Janus-like films of CNWs-SUC18/75+GO(4:1) also exhibited thermo-reversible property. The spherical structures on the top surface disappeared after heattreatment at $60{ }^{\circ} \mathrm{C}$ and recovered as flat donut-like particles after cooling down to room temperature (Figure 4d). After such treatments, the middle part of donut-like particle collapsed and formed a circular structure with lamellar nanoplates on the surface (Figure 4e). In addition, the surface still maintained its high hydrophobicity with a SWCA of $147 \pm 2^{\circ}$.

Compared with the self-assembly of solely CNWs-SU-C18/75, the coassembly of CNWs-SU-C18/75 and GO resulted in more spherical structures formed on the top surface of films, which should be attributed to the heterogeneous nucleation of CNWsSU-C18 in bulk suspensions where the GO act as heterogeneous nuclei. Generally speaking, the free energy barrier of heterogeneous nucleation is much lower than that of homogeneous nucleation. Thus, the incorporation of GO in suspensions will strongly enhance the nucleation of CNWs-SU-C18 in bulk suspension, giving rise to more microspheres on the top of the thin films. In addition, the bottom layer contained more GO according to the measurements of the oxygen and sulfur contents (Figure S12, Supporting Information). The oxygen content on the bottom surface is obviously higher than that of the top surface, but the sulfur content on the bottom surface is obviously lower than that of the top surface. Therefore, the GO nanosheets in the suspension should have primarily aggregated and precipitated constituting majorly the bottom surface, as the concentration of mixtures started to increase during the initial evaporation period. With further evaporation of THF, CNWs that better suspended started to self-assemble on the top layers of the films. This selective process led to hybrid Janus-like films with distinct chemical compositions in the top (mainly CNWs) and bottom surface (majorly GO). Moreover, this separate distribution of CNWs and GO generated rough top surface with attached microparticles and smooth bottom surface.

Furthermore, the content of GO in the composites affected the self-assembly process of CNWs. In particular, less microsized spherical particles were formed on the top surface of the hybrid Janus-like films, if the mass ratio of CNWs-SU-C18/75 to GO was varied from 4:1 to 8:1, 2:1 or 1:2 (Figures S13-S15, Supporting Information). We speculate that a reduction of GO content will reduce the concentration of heterogeneous nuclei, resulting in fewer microparticles. At high concentrations, in turn, GO might aggregate into clusters and thereby reduce the surface available for heterogeneous nucleation, also giving rise to fewer microparticles.

The thermoreversible properties of these as-prepared sphere-like structures on the top surface of the Janus-like films of CNWs-SU-C18/75+GO(4:1) were also studied with LSM (Figure 5). The height of the initial microparticles was about $14.6 \pm 1.2 \mu \mathrm{m}$ and the diameter was averagely $12.8 \pm 1.9 \mu \mathrm{m}$ according to 3D LSM measurements. A donut-like structure formed and grew gradually after cooling down from heat-treatment at $60{ }^{\circ} \mathrm{C}$ and maintaining at room temperature for $5 \mathrm{~min}$. These donuts had a height and wall thickness of about 7.1 and $4.9 \mu \mathrm{m}$, respectively (Figure $5 \mathrm{~b}$ ). After $3 \mathrm{~h}$, the height and the wall thickness of the donut-like structure increased to 9.3 and $5.3 \mu \mathrm{m}$, respectively. After $24 \mathrm{~h}$, the height of the flat donuts further increased to $\approx 10.3 \mu \mathrm{m}$ and the wall thickness reached around 5.9 (Figure $5 \mathrm{~d}$ ).

Therefore, similar to the CNWs-SU-C18/75 films, CNWsSU-C18/75+GO(4:1) films also contained thermoresponsive microparticles with their morphology changing into donutlike structures after heating-cooling treatment, but their density was still higher than those on CNWs-SU-C18/75 films. 

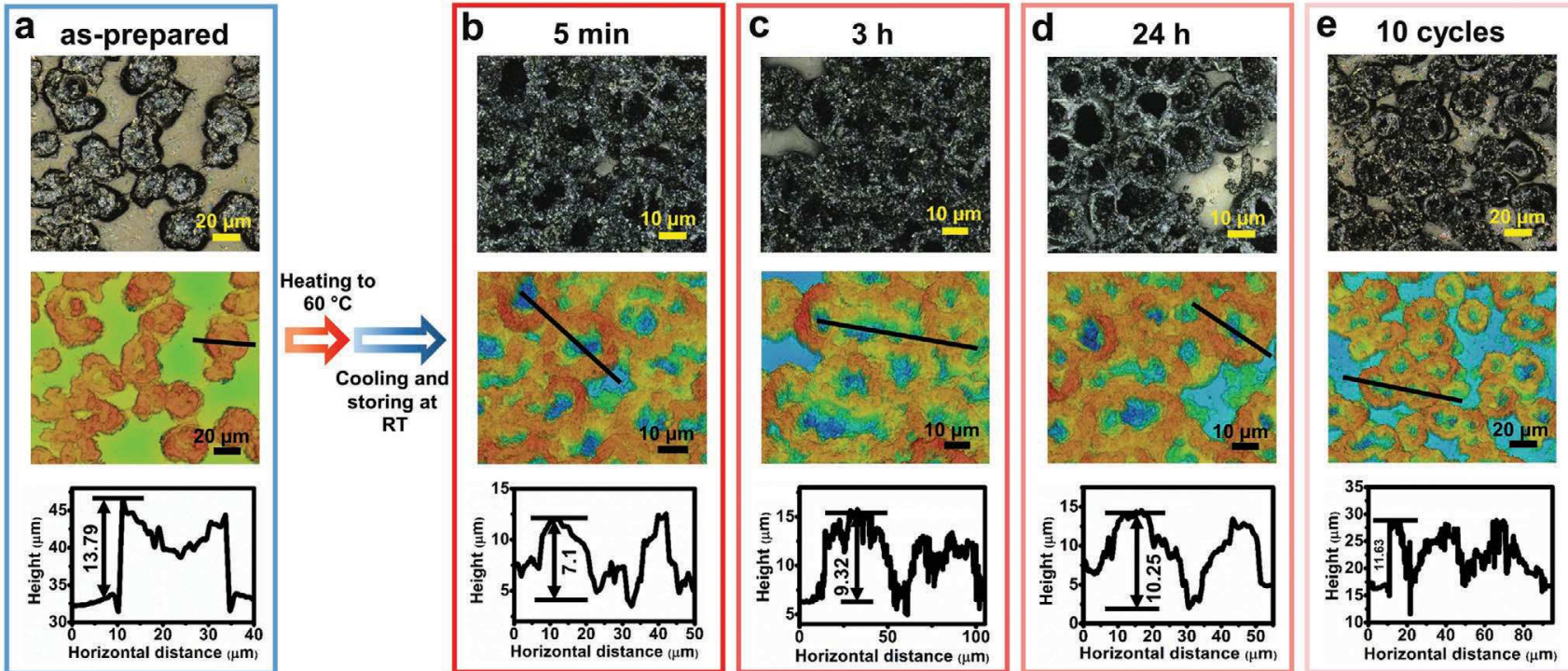

Figure 5. Thermoreversible properties of CNWs-SU-C18/75+GO(4:1) film. a) Representative LSM image, 3D LSM image, and height profile of the CNWs-SU-C18/75+GO(4:1) film. b-d) Time-dependent LSM images, 3D LSM images, and height profiles of the self-assembled structures after cooling down from $60^{\circ} \mathrm{C}$ to room temperature and further aging time of $5 \mathrm{~min}, 3 \mathrm{~h}$, and $24 \mathrm{~h}$, respectively. e) LSM image, 3D LSM image, and height profile of the CNWs-SU-C18/75+GO(4:1) film after 10 cycles of heating-cooling process. The images and height profile after the 10th cycle were recorded after maintaining at room temperature for $2 \mathrm{~d}$. RT: room temperature.

It is worth noting that the density of microparticles on the CNWs-SU-C18/75+GO(4:1) film was higher than those on CNWs-SU-C18/75 film before heating-cooling treatment. Moreover, no significant fatigue of the donut-like morphology was observed after a further 10 cycles of heating-cooling treatment (Figure 5e). In addition, the average height of the particles was higher than $10 \mu \mathrm{m}$ after complete aging, which was greater than the heights of the donuts on CNWs-SU-C18/75 films. This is mainly due to the collapse of the middle part of microspheres which formed the donut-like structures with higher edges. The presence of such higher donut-like microparticles in higher densities resulted in stronger hydrophobicity on the top surface of CNWs-SU-C18/75+GO(4:1) Janus-like films than on CNWsSU-C18/75 films (Figure 4b,d).

In addition to the thermoreversible behaviors of the micro/nanostructures on the top surface, these Janus-like films also exhibited solvent-responsive properties. To study this responsive behavior, stripes with a dimension of $2 \mathrm{~mm} \times 6 \mathrm{~mm}$ (width $\times$ length) were cut from the Janus-like films of CNWs-SU-C18/75+GO(4:1). The thickness of the films was generally adjusted to be around $30 \mu \mathrm{m}$. The actuation experiments were performed in THF and water, because THF and water are swelling and nonswelling solvents for CNWsSU-C18/75+GO(4:1), respectively. As soon as the stripes were placed in the THF solution, the straight films instantly rolled up into highly curled films with a bending angle of around $692^{\circ}$ (Figure 6a and Figure S16, Supporting Information). This process was very fast with rapidly increasing bending angles and finished within $6 \mathrm{~s}$ (Figure $6 \mathrm{c}$ and Movie S2, Supporting Information). When the curled stripes immersed into water, they responded by opening up with decreasing bending angles and became straight within $20 \mathrm{~s}$ (Figure 6b,c). The curling time of the stripe actuator was generally shorter in THF than the flattening time in water. Moreover, this rolling-up and flattening process of these Janus-like film stripes was robust and reversible. They maintained the fast bending performance even after 25 runs, by alternatively placing these stripes in THF and water under ambient conditions (Movie S3, Supporting Information). The actuation experiments were also performed in diverse THF-water mixtures, for instance, the THF-water mixture with the volume ratio of 3 to 1 . In such mixtures, the bending behaviors of the Janus-like films were weakened with lower reaction speed and much smaller curvature (Figure S17 and Movie S4, Supporting Information). The bending of the films was mainly driven by the swelling of the CNWs region with adsorbed THF. Decreasing the concentration of THF resulted in a slower swelling of the CNWs sides of the film, thus leading to the weakening of the bending behaviors of the films.

The addition of GO resulted in the hybrid Janus-like film with higher rigidity (Figure S18 and Table S1, Supporting Information). With the mass ratio of $\mathrm{CNWs}$ to $\mathrm{GO}$ in hybrid Janus-like films decreasing from 4:1 to 2:1 and 1:2, and thus increasing contents of GO, the responsiveness of the Januslike films in THF was strongly weakened (Figures S19 and S20, and Movies S5 and S6, Supporting Information). In contrast, when the mass ratio of CNWs to GO in Janus-like films was 8:1, and thus with a lower content of GO, the response speed was fast in the first cycles and decreased afterward due to the very low rigidity of the films (Figure S21 and Movie S7, Supporting Information). Moreover, the Janus-like films from solely CNWsSU-C18/75 did not show bending performance due to the good dispersibility of CNWs-SU-C18/75 in THF result in the very low rigidity of the Janus-like films.

Figure $6 \mathrm{~d}$ shows the SEM image of the top surface of the CNWs-SU-C18/75+GO(4:1) Janus-like films after dipping in 

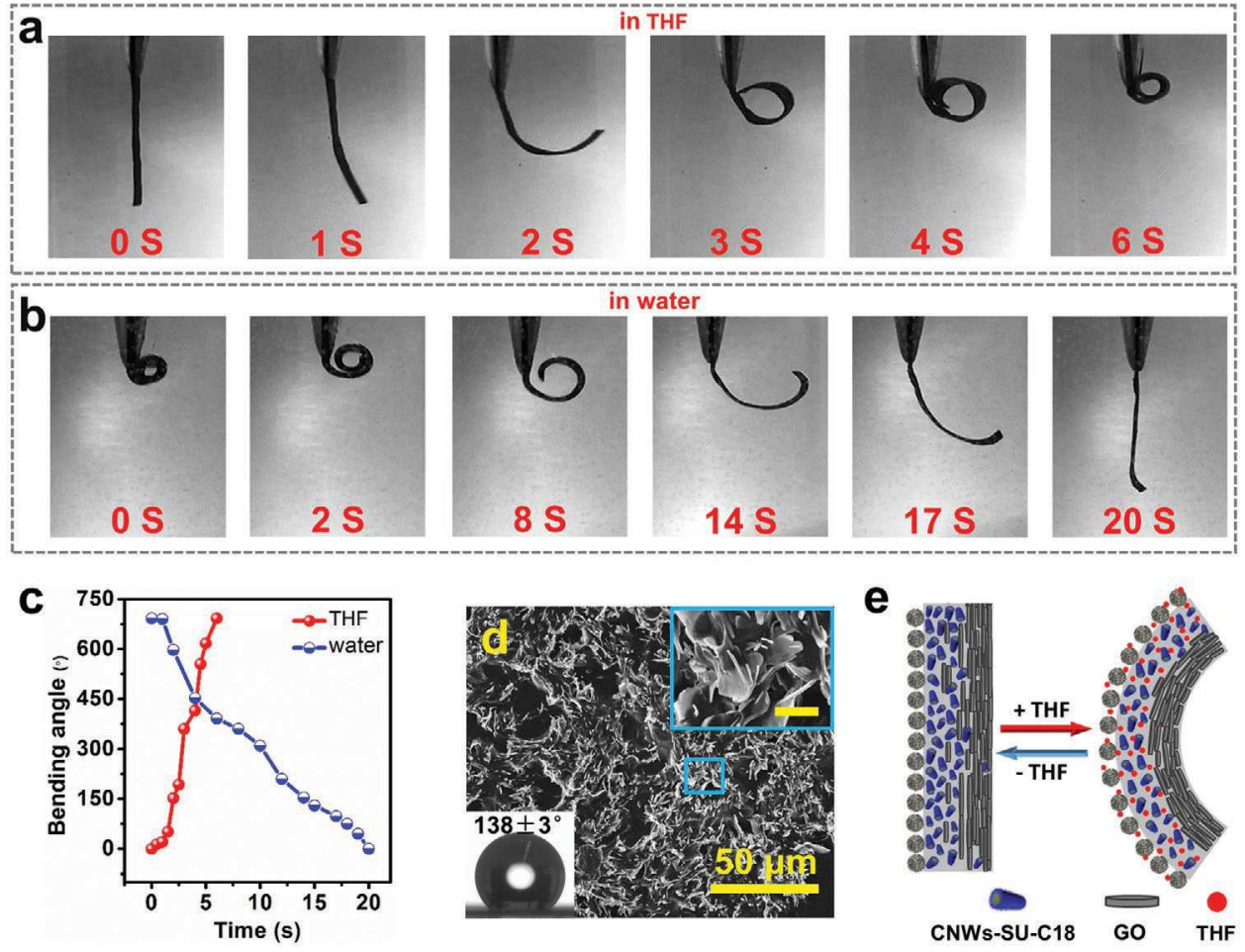

Figure 6. Responsive behaviors of CNWs-SU-C18/75+CO(4:1) Janus-like films. Snapshots showing reversible bending processes of the Janus-like film stripes in a) THF after $0,1,2,3,4$, and $6 \mathrm{~s}$ as well as b) water after $0,2,8,14,17$, and $20 \mathrm{~s}$. c) The bending angle variation as results of time for the actuation in THF and water. d) SEM image of the top surface of a CNWs-SU-C18/75+GO(4:1) Janus-like film after the exposure in THF. The top right inset shows the magnified region as in (d) with the scale bar of $5 \mu \mathrm{m}$. The bottom left inset shows SWCA on the top surface. e) Schematic illustration for the bending behaviors of the CNWs-SU-C18/75+GO Janus-like films.

THF and subsequent drying. The microsized particles vanished and only random lamellate nanosheets formed, leading to smaller SWCA of $138 \pm 3^{\circ}$. On the contrary, the exposure of the films in THF had no influence on the smooth bottom surface and the SWCA of the bottom surface remained with $98 \pm 2^{\circ}$ (Figure S22, Supporting Information). Therefore, THF had a strong impact on the CNWs region but only a negligible effect on the GO region of the films. When dipped in THF, CNWs regions very quickly absorbed THF and swelled, and the films bent towards the GO side. By immersing in water, the films turned flat again (Figure 6b,e), because THF diffused from Janus-like films into water and water only did minimum damage to the superhydrophobic top surface containing mainly CNWs. Therefore, the asymmetric structures in the Janus-like films and distinct swelling behaviors of CNWs and GO led to curling and flattening behaviors of these hybrid Janus-like films in diverse solvents.

The high degree of bending deformation or curling movement of such film actuators is interesting for diverse applications, such as self-walking devices, controlled release, microvalves, bimetal thermometers, and sensors. ${ }^{[19]}$ Most of the actuators have bi- or trilayer configurations, in which a stimuli-responsive layer adheres to a nonresponsive flexible support. Multilayer actuators often suffer from possible delamination of the two layers after long-term exposure to diverse surroundings, resulting in a reduced lifetime. Actuators with a single-layer configuration are therefore preferable because of their durability and long-term ability to withstand a range of chemicals. ${ }^{[20]}$ Thus, our work not only extends the fundamental knowledge of the self-assembly of CNWs, but also provides a facile one-step approach for fabricating single-layer Janus-like films with solvent-responsive properties, which have various potential applications.

\section{Conclusion}

In conclusion, we developed a facile one-step method to fabricate multiresponsive Janus-like films via the evaporation-driven self-assembly of surface-acylated CNWs bearing long alkane chains with or without GO. The heterogeneous nucleation of nanowhiskers on rough Teflon substrates favors the formation of thin films, whereas the homogeneous nucleation of residual nanowhiskers in bulk solutions promotes the growth of patchy microspheres that are composed of a large number of lamellar nanoplates. The incorporation of GO in the solution lowers the free-energy barrier of homogeneous nucleation and promote the formation of more microparticles on the top surface. Januslike films were obtained with diverse morphologies and wettabilities on both surfaces, while GO enhanced these differences on both surfaces. In particular, the microspheres on the top surface of Janus-like films show thermoreversible and reconstructable time-dependent donut structures in the dry state. The structural difference between the top and bottom surfaces 
of GO-containing Janus-like films facilitates the solvent-responsive curling actuation in different solvents with fast reversible bending motions for many cycles. These findings not only extend our understanding of the self-assembly of anisotropic nanorods dominated by one mechanism toward those with combined mechanisms, e.g. the interfacial activity on (liquidsubstrate) interfaces and the bulk structure formation in solutions, but also provide inspirations in designing and fabricating other functional Janus-like materials.

\section{Experimental Section}

Materials: Microcrystalline cellulose (MCC) with an average particle size of $50 \mu \mathrm{m}, 10$-undecenoyl chloride, 1-octadecanethiol, and graphene flakes (325 mesh) were purchased from Sigma-Aldrich Chemie $\mathrm{GmbH}$ (Steinheim, Germany). Dry pyridine and tetrahydrofuran (THF) were brought from Th. Geyer $\mathrm{GmbH} \&$ Co. KG (Renningen, Germany). Other chemicals are all of analytical grade and used as received. Deionized water (DI water) was used in all experiments.

Synthesis of Cellulose Nanowhiskers with Surface-Attached 10-Undecenoyl Groups (CNWs-SU): CNWs-SU were synthesized according to published procedures with a few modifications. ${ }^{[6]}$ Briefly, $1 \mathrm{~g}$ of MCC was fed into a three-necked flask. Then, $30 \mathrm{~mL}$ pyridine was added and $\mathrm{MCC}$ dispersed therein by stirring. After the mixture was heated up to $50^{\circ} \mathrm{C}, 2.73 \mathrm{~mL}$ 10 -undecenoyl chloride $(2 \mathrm{~mol}$ acid chloride per $\mathrm{mol}$ anhydroglucose units of cellulose) was added to the cellulose suspension under stirring. $n$, the reaction mixture was poured into $200 \mathrm{~mL}$ methanol immediately. The precipitate was separated by centrifugation. Thereafter, the product was purified by a repeated dispersion and precipitation process using THF and methanol, respectively, before the product was dispersed in THF. Then, the suspension was centrifuged for $10 \mathrm{~h}$ at $14000 \mathrm{rpm}$ at $4^{\circ} \mathrm{C}$. After the centrifugation, the supernatant was removed and the solid was dispersed in THF again, which was centrifuged for $20 \mathrm{~min}$ at $3000 \mathrm{rpm}$ at $20{ }^{\circ} \mathrm{C}$ to remove the microscaled segments. Finally, the purified product was dispersed in THF for further use. The corresponding yield of CNWs was $11.6 \pm 2.4 \%$. The DS of the obtained CNWs-SU was determined to be 1.32 .

Postmodification of CNWs-SU via Thiol-Ene Reaction with 1-Octadecanethiol to Synthesize CNWs-SU-C18: 1-octadecanethiol (1, 0.75, and $0.25 \mathrm{~mol}$ per mol $\mathrm{C}=\mathrm{C}$ double bonds) was added into THFsuspension of CNWs-SU (10 mg mL $\left.\mathrm{mL}^{-1}\right)$. The mixture was exposed to UV light $\left(320-400 \mathrm{~nm}\right.$ with the intensity of $\left.\approx 15 \mathrm{~mW} \mathrm{~cm}{ }^{-1}\right)$ for $3 \mathrm{~h}$ at room temperature under stirring. The obtained CNWs-SU-C18 were referred to as CNWs-SU-C18/100, CNWs-SU-C18/75, and CNWs-SU-C18/25, respectively. After the reaction, all mixtures were transferred in dialysis membrane (with a molecular weight cutoff of $3500 \mathrm{Da}$ ) and kept in 5 volumes of THF for $12 \mathrm{~h}$. Then, THF was changed twice after every $12 \mathrm{~h}$. Thereafter, the dialyzed products were centrifuged for $20 \mathrm{~min}$ at $3000 \mathrm{rpm}$ at $20^{\circ} \mathrm{C}$ to remove the aggregates. Finally, all purified CNWs$\mathrm{SU}-\mathrm{C} 18$ were dispersed in THF for further use.

Synthesis of Graphene Oxide: GO suspension was prepared via the modified Hummers method. ${ }^{[18]} 1.5 \mathrm{~g}$ graphene flakes were fed into a three-necked flask and a 9:1 mixture of concentrated $\mathrm{H}_{2} \mathrm{SO}_{4} / \mathrm{H}_{3} \mathrm{PO}_{4}$ (180:20 in V/V) was added. Then, $9 \mathrm{~g} \mathrm{KMnO}_{4}$ was added slowly while stirring at room temperature. The reaction mixture was then heated up to $50{ }^{\circ} \mathrm{C}$ and stirred for $12 \mathrm{~h}$. After the reaction, the flask was removed from the oil bath and cooled down to room temperature. The reaction mixture was slowly added to the ice $(\approx 300 \mathrm{~mL})$ with $1.5 \mathrm{~mL}$ of $30 \%$ $\mathrm{H}_{2} \mathrm{O}_{2}$ and then was stored for $12 \mathrm{~h}$ at room temperature. Thereafter, the suspension was centrifuged for $60 \mathrm{~min}$ at $4000 \mathrm{rpm}$ and $25^{\circ} \mathrm{C}$ to separate the solids from the liquid phase. The remaining solid materials were then washed in succession with $100 \mathrm{~mL}$ of water, $100 \mathrm{~mL}$ of $30 \%$ $\mathrm{HCl}$, and $100 \mathrm{~mL}$ of ethanol $(2 \times)$. For each washing step, the mixture was centrifuged for $30 \mathrm{~min}$ at $4000 \mathrm{rpm}$ and $25^{\circ} \mathrm{C}$ to separate the solid from the liquid phase. Finally, the solid phase was suspended in $50 \mathrm{~mL}$ of ethanol. The product suspension was left under room temperature for two days, so the ethanol evaporated.

Solvent Casting of CNWs-SU-C18 and CNWs-SU-C18+CO for SelfAssembled Self-Standing Films: The films were obtained after the deposition of $10 \mathrm{~mL}$ of homogeneous THF suspensions of CNWs-SU, CNWs-SU-C18/100, CNWs-SU-C18/75, and CNWs-SU-C18/25 with the concentration of $10 \mathrm{mg} \mathrm{mL}^{-1}$ on round Teflon molds (diameter: $5 \mathrm{~cm}$ ) using a solvent casting technique. After $15 \mathrm{~h}$ of drying at room temperature, the films were removed from Teflon substrates for further characterization. The films of CNWs-SU-C18/75 mixed with various weight ratios of GO (2:1, 4:1, 8:1, 1:2) were also obtained by solvent casting. $10 \mathrm{~mL}$ of mixed CNWs-SU-C18/75 and GO suspensions with the concentration of $10 \mathrm{mg} \mathrm{mL}^{-1}$ were transferred into equal round Teflon molds (diameter: $5 \mathrm{~cm}$ ). After $15 \mathrm{~h}$ of drying at room temperature, the films were removed from Teflon substrates for further characterization.

Elemental Analysis: The contents of carbon, hydrogen, and sulfur were determined with an Elemental Analyzer 4.1 vario EL III (Elementar, Germany). DS of corresponding groups was calculated according to the carbon contents, as the following formula:[2]]

$$
\mathrm{DS}=\frac{5.13766-11.5592 \times C \%}{0.996863 \times C \%-0.856277 \times n+n \times C \%}
$$

where $\mathrm{C} \%$ is the content of elemental carbon and $n$ is the number of carbon atoms in the acyl moieties, respectively.

Solid-State $13 \mathrm{C}$ CP-MAS NMR Spectroscopy: Solid-state ${ }^{13} \mathrm{C}$ CP-MAS NMR measurements for all samples were acquired at ambient conditions using a Bruker AVANCE IIIHD 800WB spectrometer with a field strength of 18.8 T. Experiments were performed using a Bruker $1.3 \mathrm{~mm}$ MAS triple resonance probe with a MAS spinning frequency of $55.555 \mathrm{kHz}$. A ramped CP-MAS sequence was used with a ${ }^{1} \mathrm{H}$ excitation power of $138.8 \mathrm{kHz}$ and a Hartman Hahn contact time of $2 \mathrm{~ms}$ with a ${ }^{13} \mathrm{C}$ inherent radio frequency (rf) field strength of $62.5 \mathrm{kHz}$ and ${ }^{1} \mathrm{H}$ rf field strength of $127.4 \mathrm{kHz}$. During the acquisition of $27 \mathrm{~ms},{ }^{7}{ }^{1} \mathrm{H}$ decoupling was performed with an inherent rf field of $14.7 \mathrm{kHz}$ employing WALTZ-16 sequence. ${ }^{[22]}$ In a typical $8 \mathrm{~h}$ experiment, 15360 scans were accumulated with a recycle delay of $2.5 \mathrm{~s}$.

The crystallinities of the samples were determined based on the solid-state ${ }^{13} \mathrm{C}$ CP-MAS NMR spectra. The individual peaks ascribed to $\mathrm{C} 4$ in crystalline and amorphous regions were extracted by a peak deconvolution process in Origin 8.5 using Gaussian function (Figure S2, Supporting Information). The crystallinity was calculated using the following equation ${ }^{[23]}$

Crystallinity $(\%)=\frac{A_{\text {cr. }}}{A_{\text {cr. }+A_{\text {am. }}}} \times 100$

where $A_{\text {cr. }}$ and $A_{\text {amm. }}$ represent the areas obtained by the integration of the fitted curves of the crystalline peak and amorphous peak in the C4 region, respectively.

Scanning Electron Microscopy: Scanning electron microscopy (SEM) images were obtained on a LEO Supra-35 high-resolution field emission scanning electron microscope (Carl Zeiss AG, Germany) at an accelerating voltage of $5 \mathrm{kV}$. A layer of carbon was coated on the surface of samples before SEM measurements.

Transmission Electron Microscope: The transmission electron microscope (TEM) observation was performed on a CM 12 Transmission Electron Microscope (Philips, Netherland). The specimen was stained by phosphotungstic acid solution ( $0.2 \mathrm{wt} \%$ in THF) for about $2 \mathrm{~min}$ to enhance the contrast.

Atomic Force Microscopy: Atomic force microscopy (AFM) topographic measurements were performed using a Cypher S AFM (Asylum Research, Santa Barbara, CA) in tapping mode in air. Samples were prepared by transferring a drop of diluted GO suspension onto freshly cleaved mica. Silicon nitride cantilevers with a tip radius of $7 \mathrm{~nm}$, nominal spring constant of $2 \mathrm{~N} \mathrm{~m}^{-1}$, and nominal resonance frequency of $70 \mathrm{kHz}$ (AC240TS-R3, Olympus) were used. Images processing and subsequent evaluation of sample geometrical parameters was performed with Gwyddion (Free Software Foundation, Inc., Boston, USA). 
Laser Scanning Microscope: Laser scanning microscope (LSM) images were obtained on a VK-X100K 3D laser scanning microscope (Keyence Corporation, Germany) under ambient conditions. The average heights and diameters of microparticles are calculated based on the measurement of around 50 microparticles in LSM images.

Static Water Contact Angles (SWCA): The wetting properties of films were measured on a Contact Angle System OCA 15EC (Dataphysics, Filderstadt, Germany). Static water contact angles were measured using the sessile drop $(4 \mu \mathrm{L})$ technique averaged over five repeats.

Statistical Analysis: Statistical analyses were performed using the Origin software. All data are presented as the mean value \pm SD.

\section{Supporting Information}

Supporting Information is available from the Wiley Online Library or from the author.

\section{Acknowledgements}

H.L. and B.P. contributed equally to this work. K.Z. thanks German Research Foundation (DFG) for the financial support for the project ZH546/2-1 and Georg-August-University of Goettingen for the start-up funding. H.L., B.P., and H.Z. thank the China Scholarship Council (CSC) for the financial support. Q.T. and M.M. acknowledge the financial support from the DFG under grant Mu1674/15-2.

Open access funding enabled and organized by Projekt DEAL.

\section{Conflict of Interest}

The authors declare no conflict of interest.

\section{Keywords}

cellulose nanowhiskers, multiresponsive Janus-like films, self-assembly, surface acylation

Received: August 11, 2020

Revised: September 15, 2020

Published online:

[1] a) Q. Wang, Z. Wang, Z. Li, J. Xiao, H. Shan, Z. Fang, L. Qi, Sci. Adv. 2017, 3, e1701183; b) M. Grzelczak, J. Vermant, E. M. Furst, L. M. Liz-Marzán, ACS Nano 2010, 4, 3591; c) C. J. Brinker, Y. Lu, A. Sellinger, H. Fan, Adv. Mater. 1999, 11, 579.

[2] a) P. Liu, C. Mai, K. Zhang, ACS Sustainable Chem. Eng. 2017, 5, 5313; b) B.-H. Sohn, J.-M. Choi, S. I. Yoo, S.-H. Yun, W.-C. Zin, J. C. Jung, M. Kanehara, T. Hirata, T. Teranishi, J. Am. Chem. Soc. 2003, 125, 6368 .

[3] M. A. S. Azizi Samir, F. Alloin, A. Dufresne, Biomacromolecules 2005, 6,612 .

[4] a) Y. Habibi, L. A. Lucia, O. J. Rojas, Chem. Rev. 2010, 110, 3479; b) J. F. Revol, H. Bradford, J. Giasson, R. H. Marchessault,
D. G. Gray, Int. J. Biol. Macromol. 1992, 14, 170; c) D. G. Gray, X. Mu, Materials 2015, 8, 7873.

[5] A. G. Dumanli, G. Kamita, J. Landman, H. van der Kooij, B. J. Glover, J. J. Baumberg, U. Steiner, S. Vignolini, Adv. Opt. Mater. 2014, 2, 646

[6] H. Liu, B. Pang, R. Garces, R. Dervisoglu, L. Chen, L. Andreas, K. Zhang, Angew. Chem. 2018, 130, 16561; , Angew. Chem., Int. Ed. 2018, 57, 16323.

[7] a) I. Siró, D. Plackett, Cellulose 2010, 17, 459; b) C. Miao, W. Y. Hamad, Cellulose 2013, 20, 2221.

[8] a) Y. Habibi, Chem. Soc. Rev. 2014, 43, 1519; b) S. Eyley, W. Thielemans, Nanoscale 2014, 6, 7764.

[9] a) H. Thérien-Aubin, A. Lukach, N. Pitch, E. Kumacheva, Angew. Chem. 2015, 127, 5710; ,Angew. Chem., Int. Ed. 2015, 54, 5618; b) B. Wicklein, A. M. Diem, A. Knöller, M. S. Cavalcante, L. Bergström, J. Bill, Z. Burghard, Adv. Funct. Mater. 2018, 28, 1704274.

[10] a) Y. Li, H. Zhu, S. Zhu, J. Wan, Z. Liu, O. Vaaland, S. Lacey, Z. Fang, H. Dai, T. Li, L. Hu, NPG Asia Mater. 2015, 7, e150; b) L. Valentini, M. Cardinali, E. Fortunati, L. Torre, J. M. Kenny, Mater. Lett. 2013, 105, 4; c) R. Xiong, K. Hu, A. M. Grant, R. Ma, W. Xu, C. Lu, X. Zhang, V. V. Tsukruk, Adv. Mater. 2016, 28, 1501; d) K. K. Sadasivuni, A. Kafy, L. Zhai, H. Ko, S. Mun, J. Kim, Small 2015, 11, 994; e) C. Zhu, S. Monti, A. P. Mathew, ACS Nano 2018, $12,7028$.

[11] H. Zhang, R. Hao, J. K. Jackson, M. Chiao, H. Yu, Chem. Commun. 2014, 50, 14843.

[12] P. Zhang, Q. Wang, R. Guo, M. Zhang, S. Wang, C. Lu, M. Xue, J. Fan, Z. He, W. Rao, Mater. Horiz. 2019, 6, 1643.

[13] A. Isogai, M. Usuda, T. Kato, T. Uryu, R. H. Atalla, Macromolecules 1989, 22, 3168

[14] S. Björklund, A. Nowacka, J. A. Bouwstra, E. Sparr, D. Topgaard, PLoS One 2013, 8, e61889.

[15] a) P. Wang, T. Zhao, R. Bian, G. Wang, H. Liu, ACS Nano 2017, 11, 12385; b) Y. Zhao, C. Yu, H. Lan, M. Cao, L. Jiang, Adv. Funct. Mater. 2017, 27, 1701466; c) R. Nishimura, K. Hyodo, H. Sawaguchi, Y. Yamamoto, Y. Nonomura, H. Mayama, S. Yokojima, S. Nakamura, K. Uchida, J. Am. Chem. Soc. 2016, 138, 10299.

[16] a) W. Hu, Phys. Rep. 2018, 747, 1; b) Q. Tang, M. Müller, C. Y. Li, W. Hu, Phys. Rev. Lett. 2019, 123, 207801.

[17] a) S. Hope, S. Kundu, C. Roy, S. Manna, A. Hansen, Front. Phys. 2015, 3, 72; b) H. Chen, C. Grey, Adv. Mater. 2008, 20, 2206; c) J. Ge, J. Lei, R. N. Zare, Nat. Nanotechnol. 2012, 7, 428.

[18] D. C. Marcano, D. V. Kosynkin, J. M. Berlin, A. Sinitskii, Z. Sun, A. Slesarev, L. B. Alemany, W. Lu, J. M. Tour, ACS Nano 2010, 4, 4806.

[19] a) S. Ling, K. Jin, Z. Qin, C. Li, K. Zheng, Y. Zhao, Q. Wang, D. L. Kaplan, M. J. Buehler, Adv. Mater. 2018, 30, 1802306; b) L. Zong, M. Li, C. Li, Adv. Mater. 2017, 29, 1604691; c) C. Lv, H. Xia, Q. Shi, G. Wang, Y.-S. Wang, Q.-D. Chen, Y.-L. Zhang, L.-Q. Liu, H.-B. Sun, Adv. Mater. Interfaces 2017, 4, 1601002.

[20] T. Kamal, S.-Y. Park, Chem. Commun. 2014, 50, 2030.

[21] C. Vaca-Garcia, M. E. Borredon, A. Gaseta, Cellulose 2001, 8, 225.

[22] a) G. Metz, X. L. Wu, S. O. Smith, J. Magn. Reson., Ser. A 1994, 110, 219; b) A. J. Shaka, J. Keeler, R. Freeman, J. Magn. Reson. 1983, 53, 313.

[23] a) S. Park, D. K. Johnson, C. I. Ishizawa, P. A. Parilla, M. F. Davis, Cellulose 2009, 16, 641; b) R. H. Newman, L. M. Davies, P. J. Harris, Plant Physiol. 1996, 111, 475. 


\section{small $^{\text {numo }}$}

\section{Supporting Information}

for Small, DOI: 10.1002/smll.202004922

Self-Assembly of Surface-Acylated Cellulose Nanowhiskers and Graphene Oxide for Multiresponsive Janus-Like Films with Time-Dependent Dry-State Structures

Huan Liu, Bo Pang, Qiyun Tang, * Marcus Müller, Hua Zhang, Riza Dervi\#o\#lu, and Kai Zhang* 


\section{WILEY-VCH}

(C) 2020 Wiley-VCH GmbH

Supporting Information

Self-Assembly of Surface-Acylated Cellulose Nanowhiskers and Graphene Oxide for Multiresponsive Janus-like Films with Time-Dependent Dry-State Structures

Huan Liu, Bo Pang, Qiyun Tang*, Marcus Müller, Hua Zhang, Riza Dervişoğlu, and Kai Zhang*

This file includes:

Supplementary Figures

Supplementary Table

Captions for Supplementary Movie S1 to S7

Other Supplementary Materials for this manuscript includes the following:

Supplementary Movie S1 to S7 


\section{WILEY-VCH}
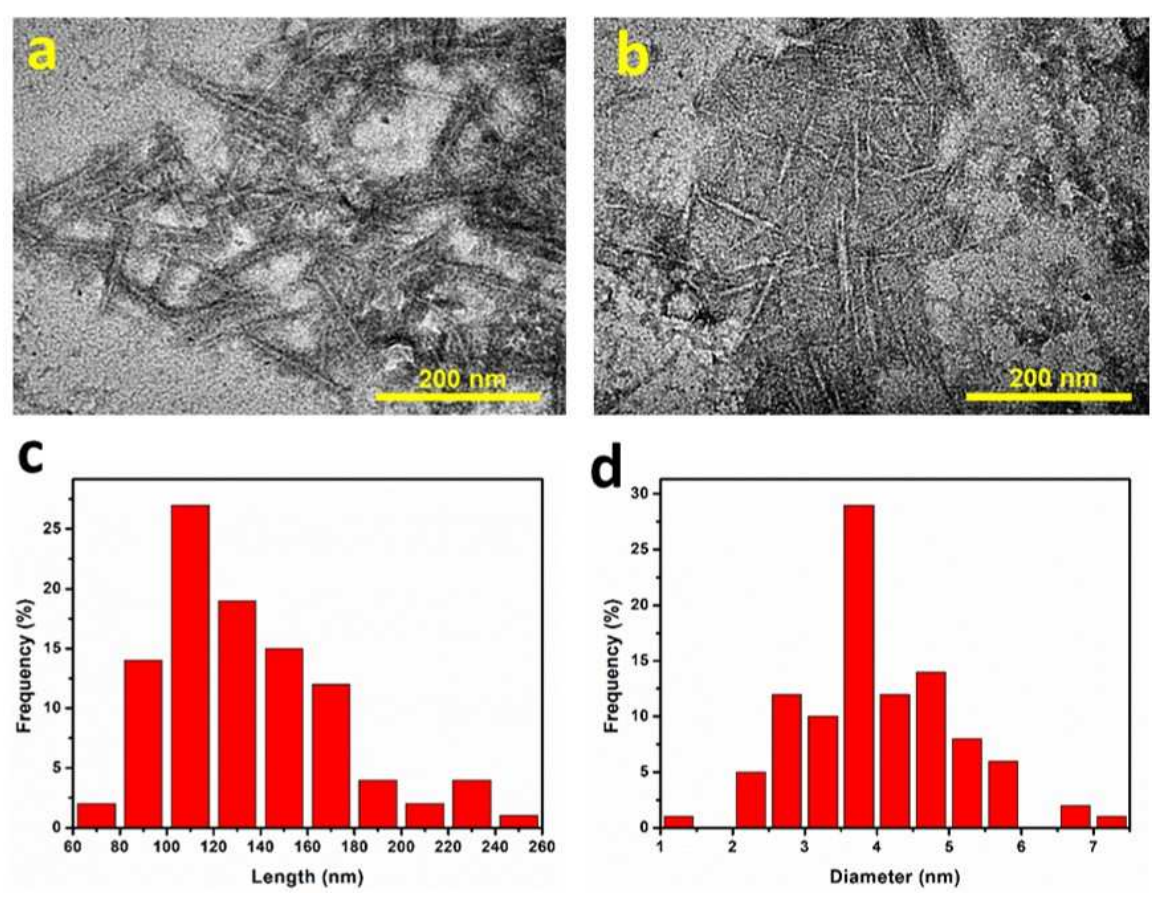

Figure S1. a, b) TEM images of CNWs-SU. c) Histogram of length distribution for CNWs-SU. d) Histogram of diameter distribution for CNWs-SU.
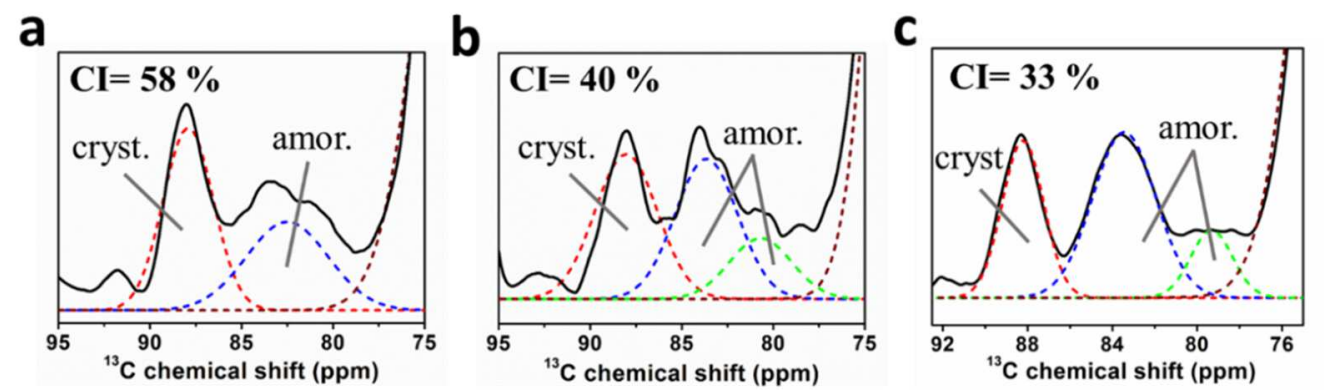

Figure S2. Peak deconvolution for the $\mathrm{C} 4$ region of solid-state CP-MAS ${ }^{13} \mathrm{C}$ NMR spectra. a) microcrystalline cellulose. b) CNWs-SU. c) CNWs-SU-C18/75. 


\section{WILEY-VCH}
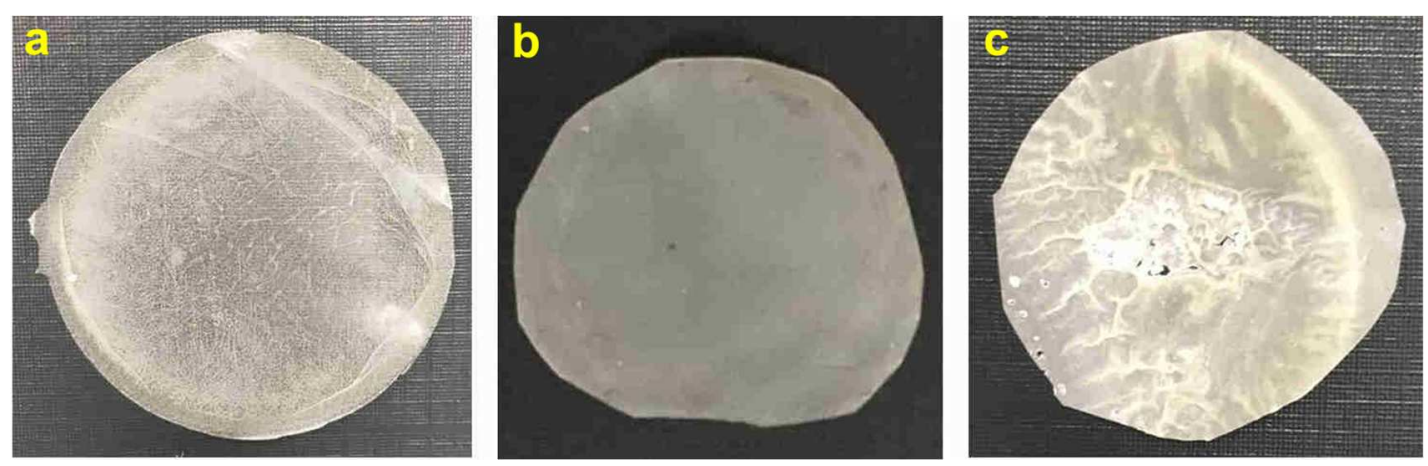

Figure S3. Photo image of the top surface of the dried films. a) CNWs-SU-C18/25. b) CNWs-SU-C18/75. c) CNWs-SU-C18/100.
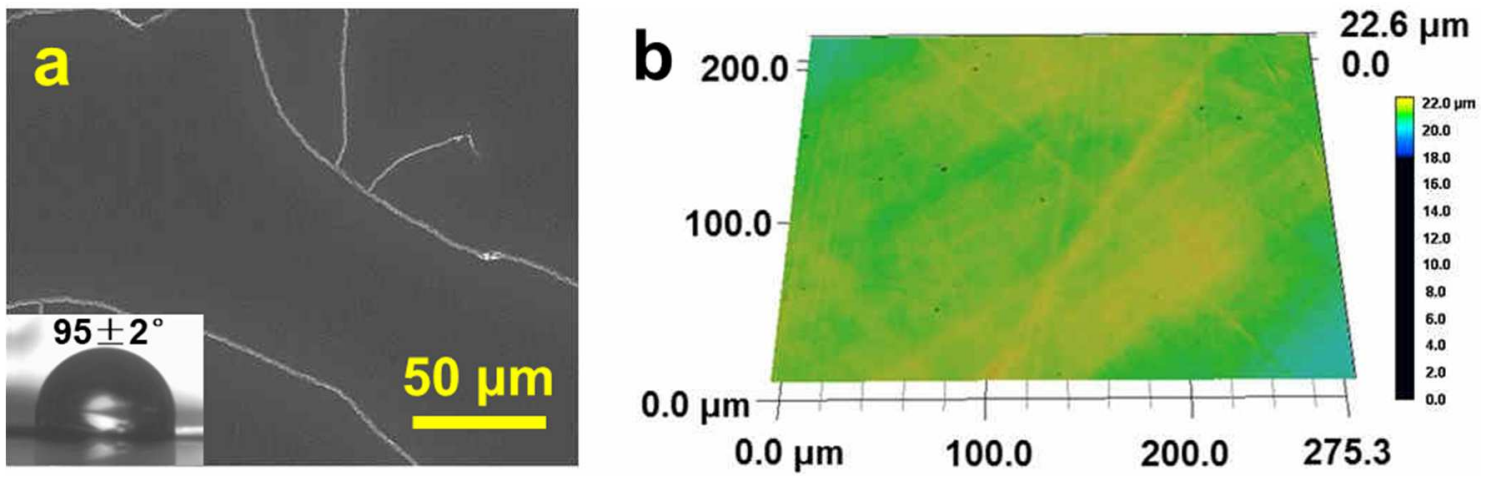

Figure S4. Morphology of the bottom surface of the CNWs-SU-C18/75 film. a) Representative SEM image of the bottom surface of the CNWs-SU-C18/75 film. The inset shows SWCA on the bottom surface. b) 3D LSM image of the bottom surface of the CNWs-SU-C18/75 film. 


\section{WILEY-VCH}
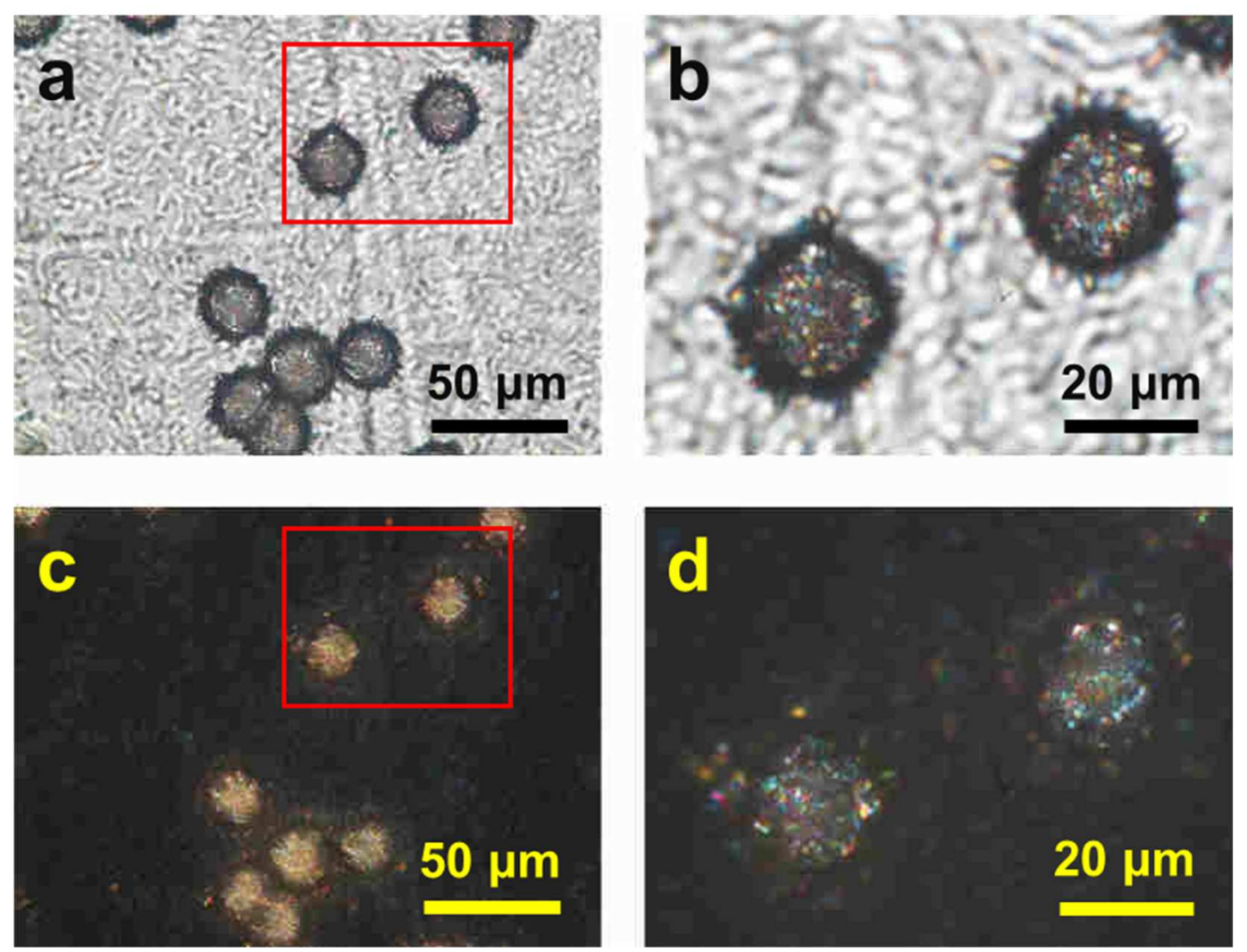

Figure S5. Microscope and polarized light microscopy (PLM) image of the self-assembled structures on the film of CNWs-SU-C18/75. a) Microscope image of the self-assembled structures on the film of CNWs-SU-C18/75. b) Microscope image of the magnified region as in (a). c) PLM image of the self-assembled structures on the film of CNWs-SU-C18/75. d) PLM image of the magnified region as in (c).
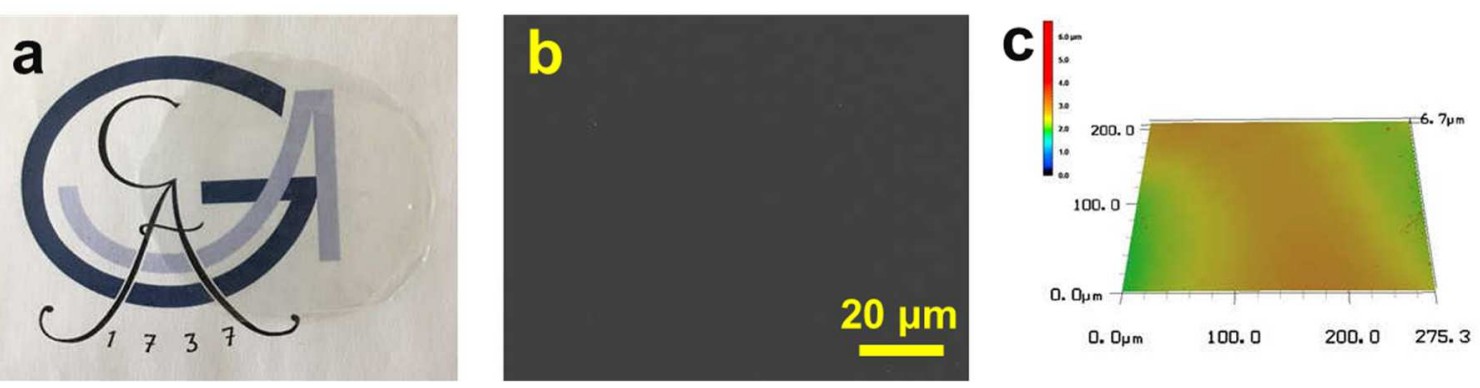

Figure S6. Dried film from CNWs-SU. a) Optical image of dried film from CNWs-SU. b) Representative SEM image and c) 3D LSM image of the CNWs-SU film. 


\section{WILEY-VCH}

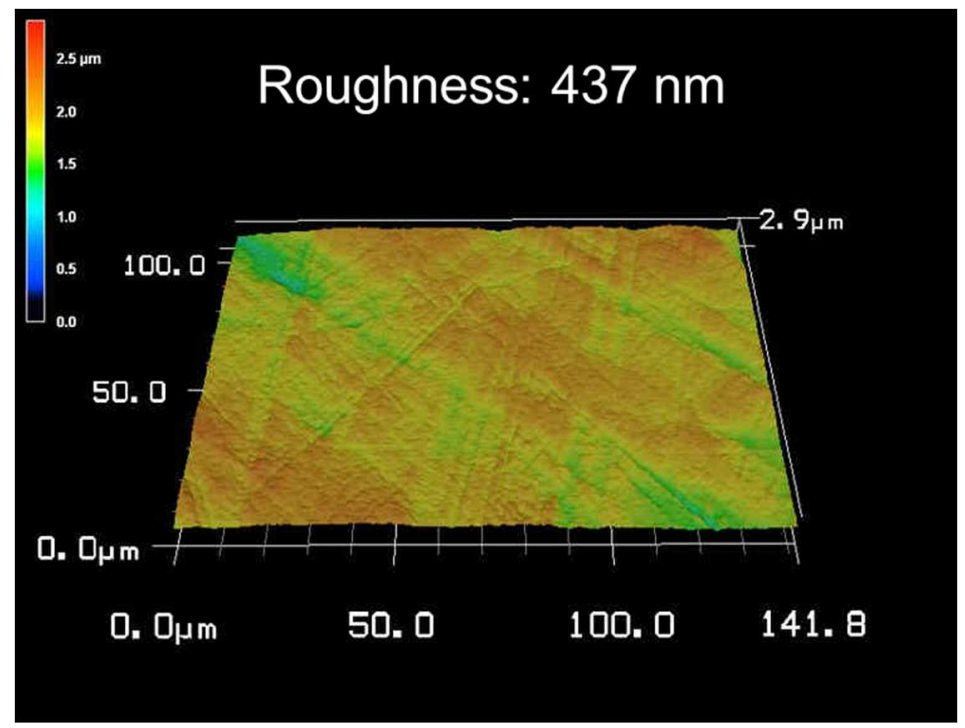

Figure S7. 3D LSM image of Teflon substrate.

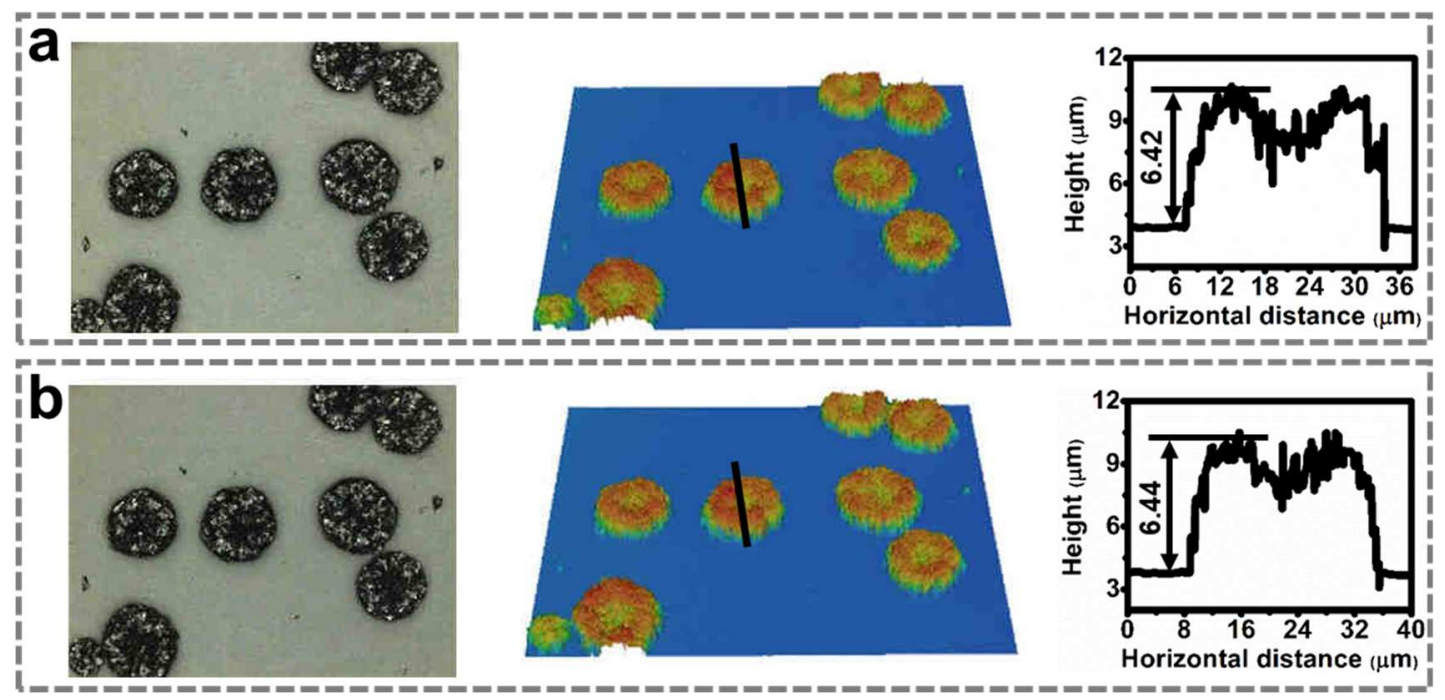

Figure S8. Thermo-reversible properties of CNWs-SU-C18/75 film. LSM image, 3D LSM image, and height profile of the CNWs-SU-C18/75 film after cooling down from $60{ }^{\circ} \mathrm{C}$ to room temperature and ageing for a) 8 days, and b) 12 days. 


\section{WILEY-VCH}
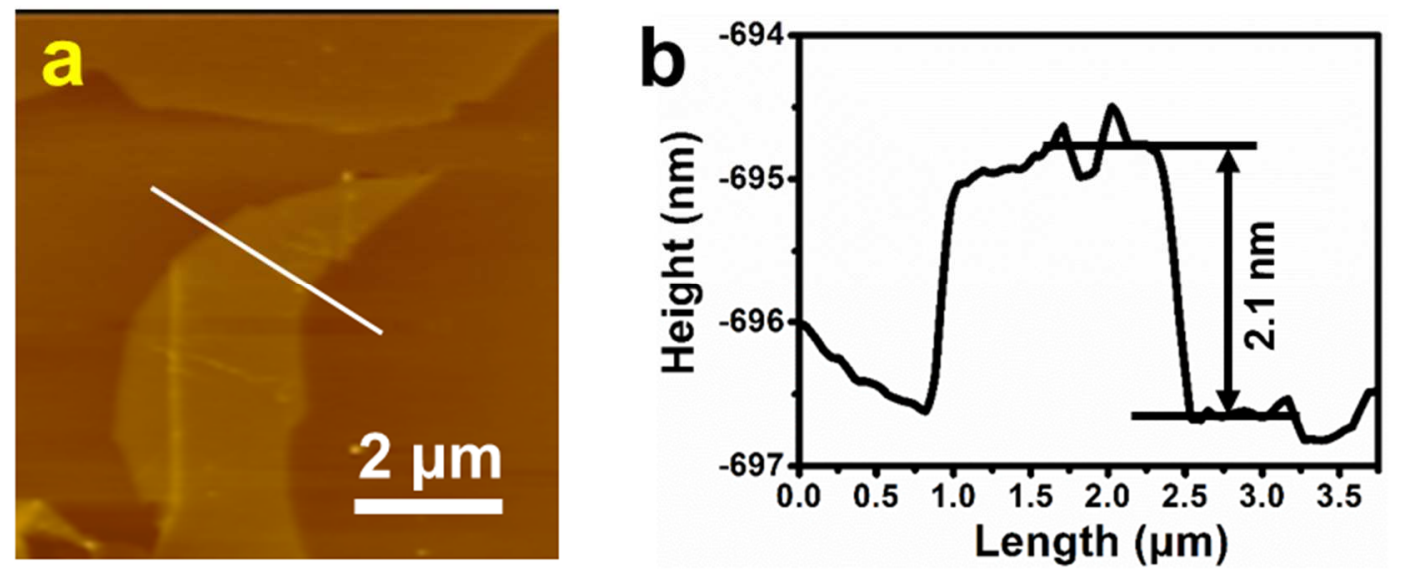

Figure S9. AFM image and cross-section analysis of GO. a) Representative AFM image of graphene oxide (GO). b) Height profile of GO as in (a).

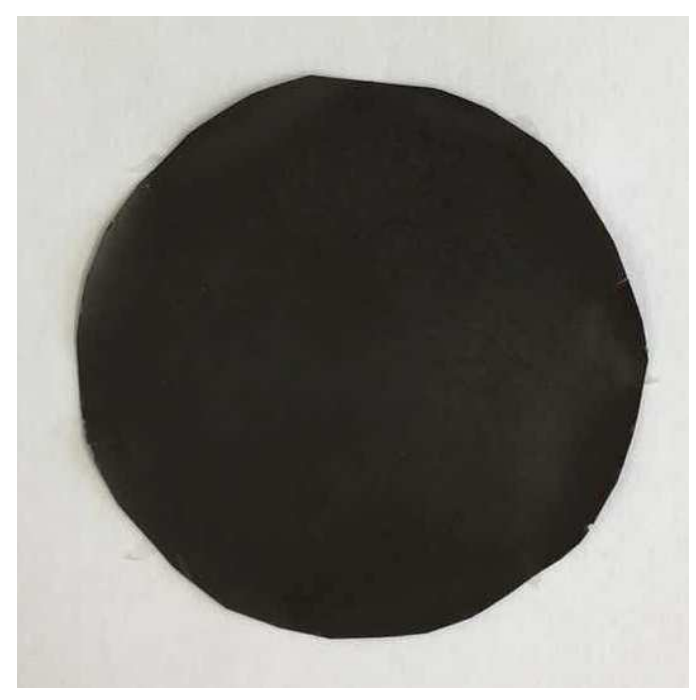

Figure S10. Photo image of the top surface of the dried CNWs-SU-C18/75+GO(4:1) film. 


\section{WILEY-VCH}
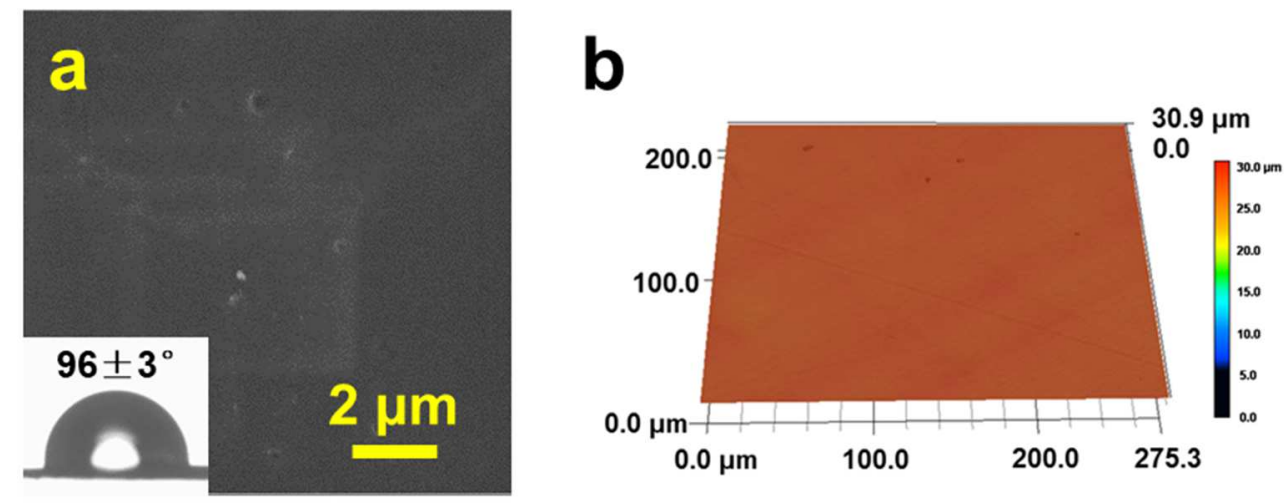

Figure S11. Morphology of the bottom surface of the CNWs-SU-C18/75+GO(4:1) film. a) Representative SEM image of the bottom surface of the CNWs-SU-C18/75+GO(4:1) film. The inset shows SWCA on the bottom surface. b) 3D LSM image of the bottom surface of the CNWs-SU-C18/75+GO(4:1) film.
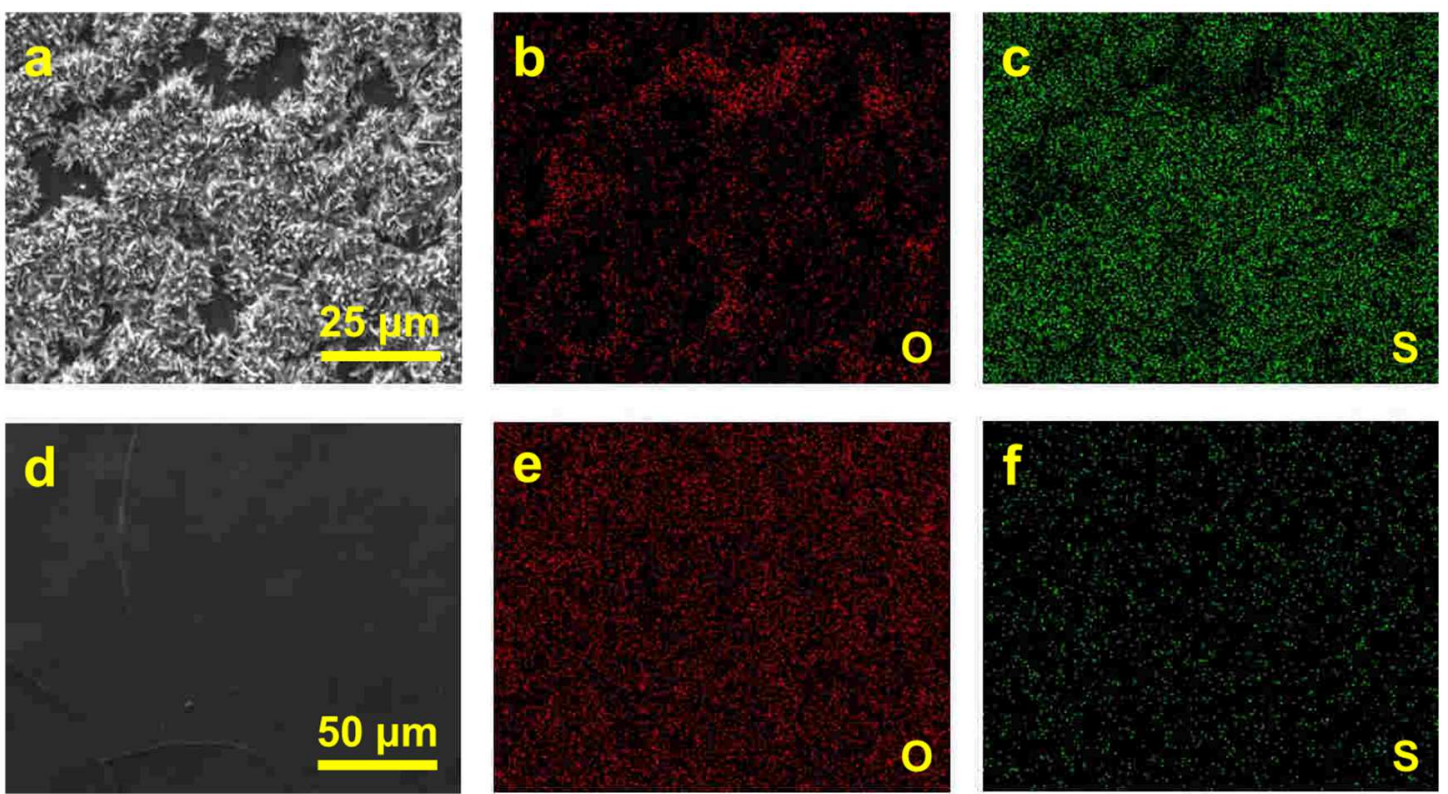

Figure S12. Elemental analysis of the CNWs-SU-C18/75+GO(4:1) film. a) SEM image of the top surface of the CNWs-SU-C18/75+GO(4:1) film. Corresponding elements mapping of $b$ ) $\mathrm{O}$ and c) S. d) SEM image of the bottom surface of the CNWs-SU-C18/75+GO(4:1) film. Corresponding elements mapping of e) $\mathrm{O}$ and $\mathrm{f}$ ) $\mathrm{S}$. 


\section{WILEY-VCH}
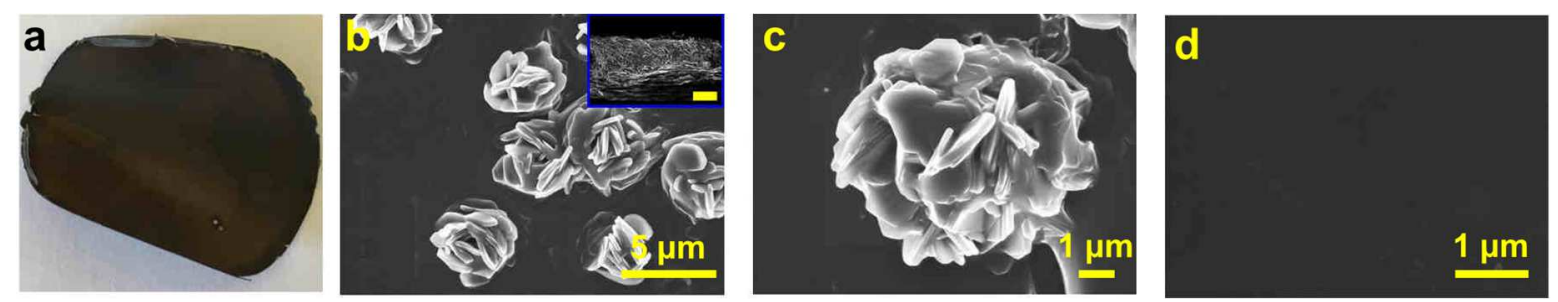

Figure S13. Characterization of dried CNWs-SU-C18/75+GO(8:1) film. a) Photo image of dried CNWs-SU-C18/75+GO(8:1) film. b) Representative SEM image of the top surface of the CNWs-SU-C18/75+GO(8:1) film. The insert shows the cross section of the film with the scale bar of $10 \mu \mathrm{m}$. c) SEM image of a microparticle with higher magnification. d) Representative SEM image of the bottom surface of the CNWs-SU-C18/75+GO(8:1) film.
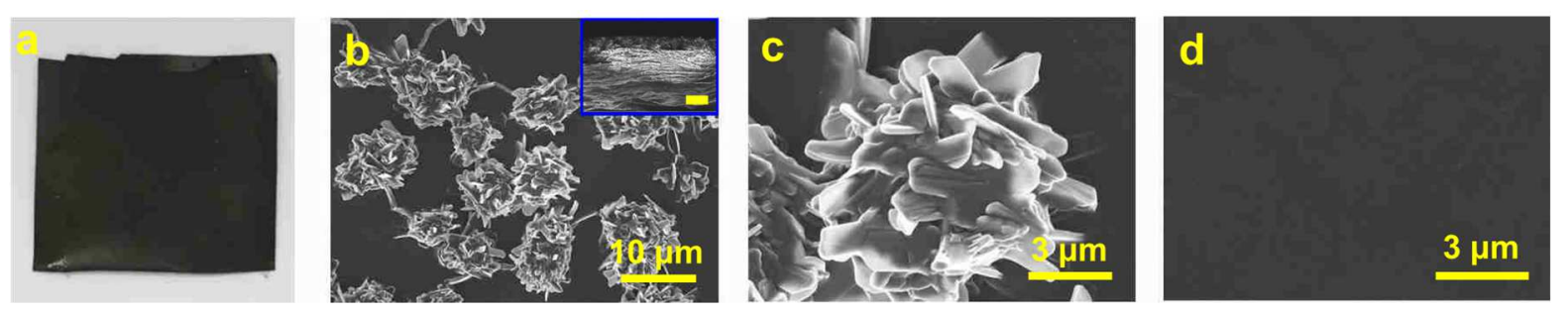

Figure S14. Characterization of dried CNWs-SU-C18/75+GO(2:1) film. a) Photo image of dried CNWs-SU-C18/75+GO(2:1) film. b) Representative SEM image of the top surface of the CNWs-SU-C18/75+GO(2:1) film. The insert shows the cross section of the film with the scale bar of $10 \mu \mathrm{m}$. c) SEM image of a microparticle with higher magnification. d) Representative SEM image of the bottom surface of the CNWs-SU-C18/75+GO(2:1) film.
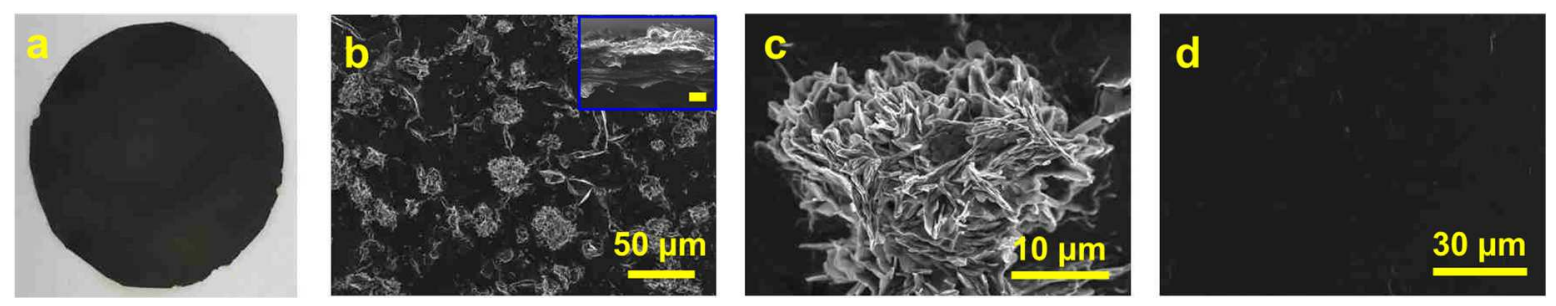

Figure S15. Characterization of dried CNWs-SU-C18/75+GO(1:2) film. a) Photo image of dried CNWs-SU-C18/75+GO(1:2) film. b) Representative SEM image of the top surface of the CNWs-SU-C18/75+GO(1:2) film. The insert shows the cross section of the film with the 


\section{WILEY-VCH}

scale bar of $5 \mu \mathrm{m}$. c) SEM image of a microparticle with higher magnification. d) Representative SEM image of the bottom surface of the CNWs-SU-C18/75+GO(1:2) film.

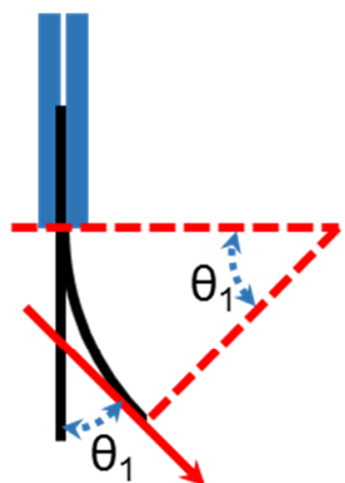

Bending angle $<90^{\circ}$

Bending angle $=\theta_{1}$

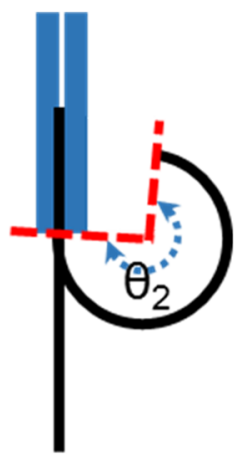

$90^{\circ}<$ Bending angle $<360^{\circ}$

Bending angle $=\theta_{2}$

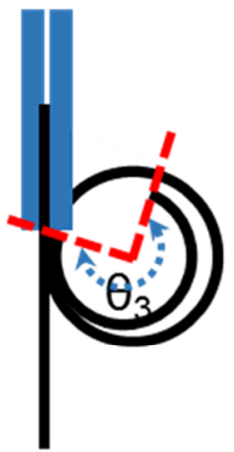

Bending angle $>360^{\circ}$

Bending angle $=\mathrm{n} \times 360^{\circ}+\theta_{3}$

( $\mathrm{n}$ is the number of bending circles)

Figure S16. Schematic illustration for the calculation of bending angles.
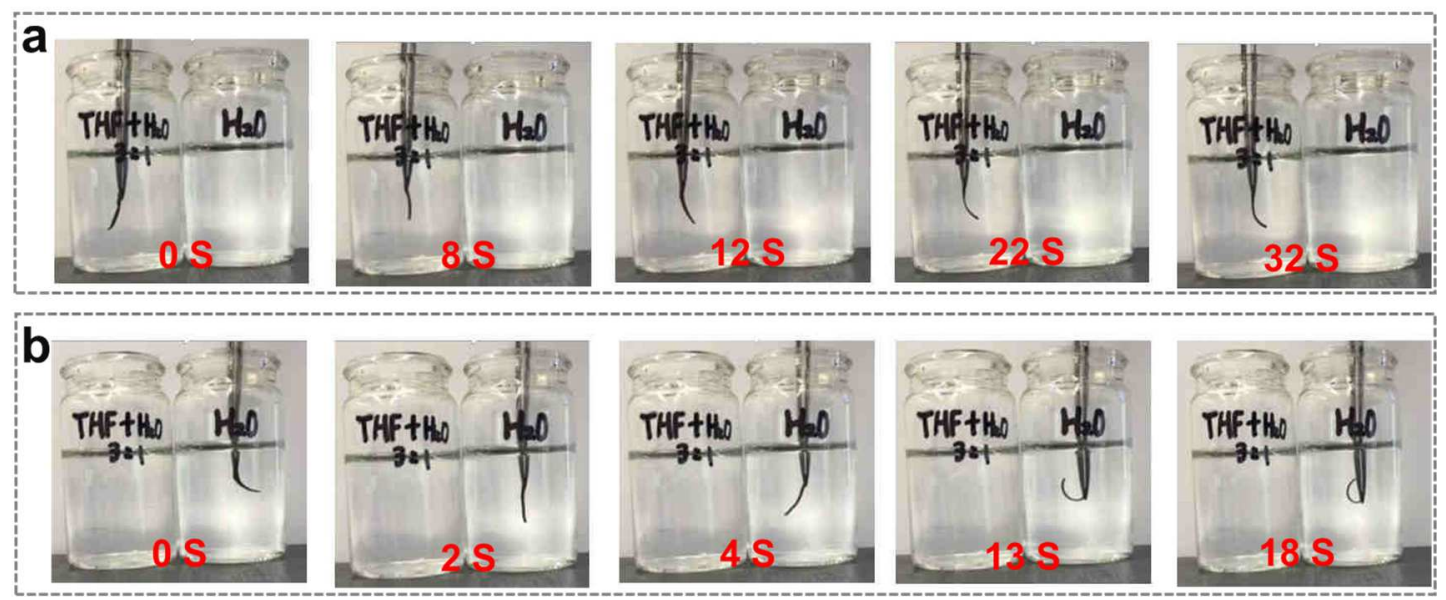

Figure S17. Responsiveness of the Janus-like film of CNWs-SU-C18/75+GO(4:1). a) Photos showing bending motions of the Janus-like stripe in THF and water $(3: 1)$ at $0,8,12,22$, and $32 \mathrm{s.b}$ ) Snapshots of shape recovery within water at $0,2,4,13$, and $18 \mathrm{~s}$. 


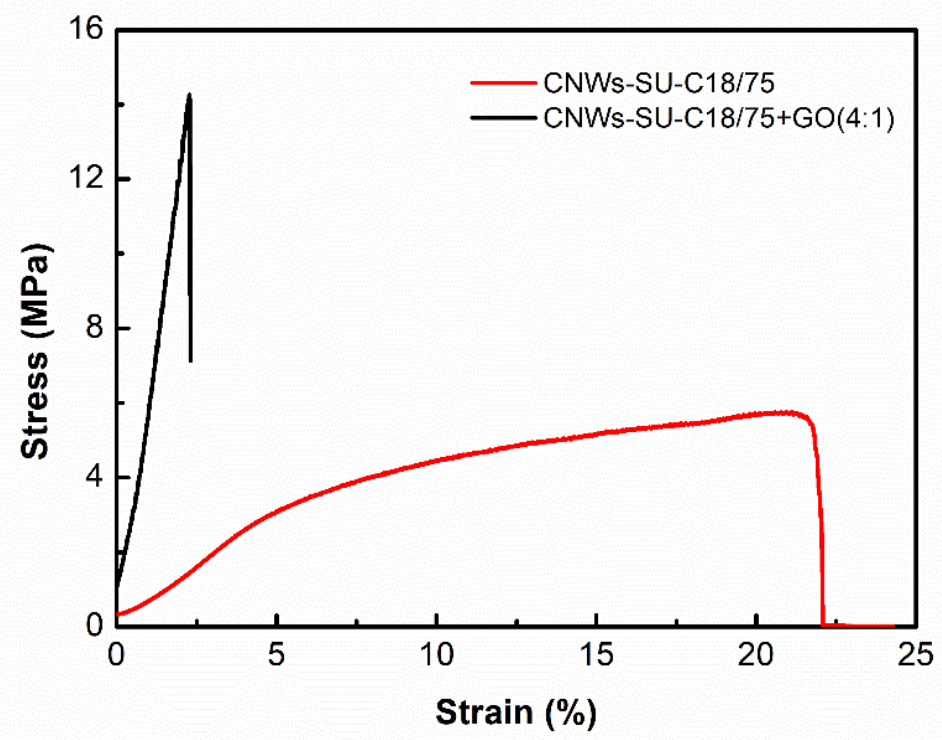

Figure S18. The stress-strain curves of CNWs-SU-C18/75 and CNWs-SU-C18/75+GO(4:1) films.

Table S1 Mechanical properties of CNWs-SU-C18/75, CNWs-SU-C18/75+GO(4:1) films, hydrophilic CNC films and neat GO films. ${ }^{1}$

\begin{tabular}{cccc}
\hline & $\begin{array}{c}\text { Tensile } \\
\text { strength } \\
(\mathrm{MPa})\end{array}$ & $\begin{array}{c}\text { Young's } \\
\text { modulus } \\
(\mathrm{MPa})\end{array}$ & $\begin{array}{c}\text { Tensile strain } \\
\text { at break } \\
(\%)\end{array}$ \\
\hline CNWs-SU-C18/75 & 5.8 & 64.3 & 22.1 \\
CNWs-SU-C18/75+GO(4:1) & 14.2 & 602.9 & 2.3 \\
Hydrophilic CNC films ${ }^{1}$ & 64 & 5960 & 1.9 \\
Neat GO films & 64.6 & 9100 & 1.0 \\
\hline
\end{tabular}

The mechanical properties of CNWs-SU-C18/75 and CNWs-SU-C18/75+GO(4:1) Janus-like films were measured. The results are summarized in Figure S19 and Table S1. For comparison, the Young's modulus and tensile strain at break of the hydrophilic CNC film ${ }^{1}$ are also listed in Table S1. One can see that the Young's modulus of Janus-like films is one (with GO) or two magnitudes (without GO) smaller than that of hydrophilic CNC films, indicating the low hardness of the films. The tensile strain at break of the films from pure CNWs-SU-C18/75 is $22.1 \%$, which is significantly larger than the $1.9 \%$ for hydrophilic CNC films. This indicates the improved toughness of CNWs-SU-C18/75 films. Both the low 


\section{WILEY-VCH}

hardness and improved toughness can be attributed to the existence of numerous long carbon chains on CNWs surfaces. The presence of GO resulted in the increase of Young's modulus up to $602.9 \mathrm{MPa}$, as well as a reduction on the tensile strain at break of the films to $2.3 \%$. The tensile strength of CNWs-SU-C18/75+GO(4:1) films was enhanced compared to pure CNWs-SU-C18/75 films, indicating the positive reinforcing effect of GO.

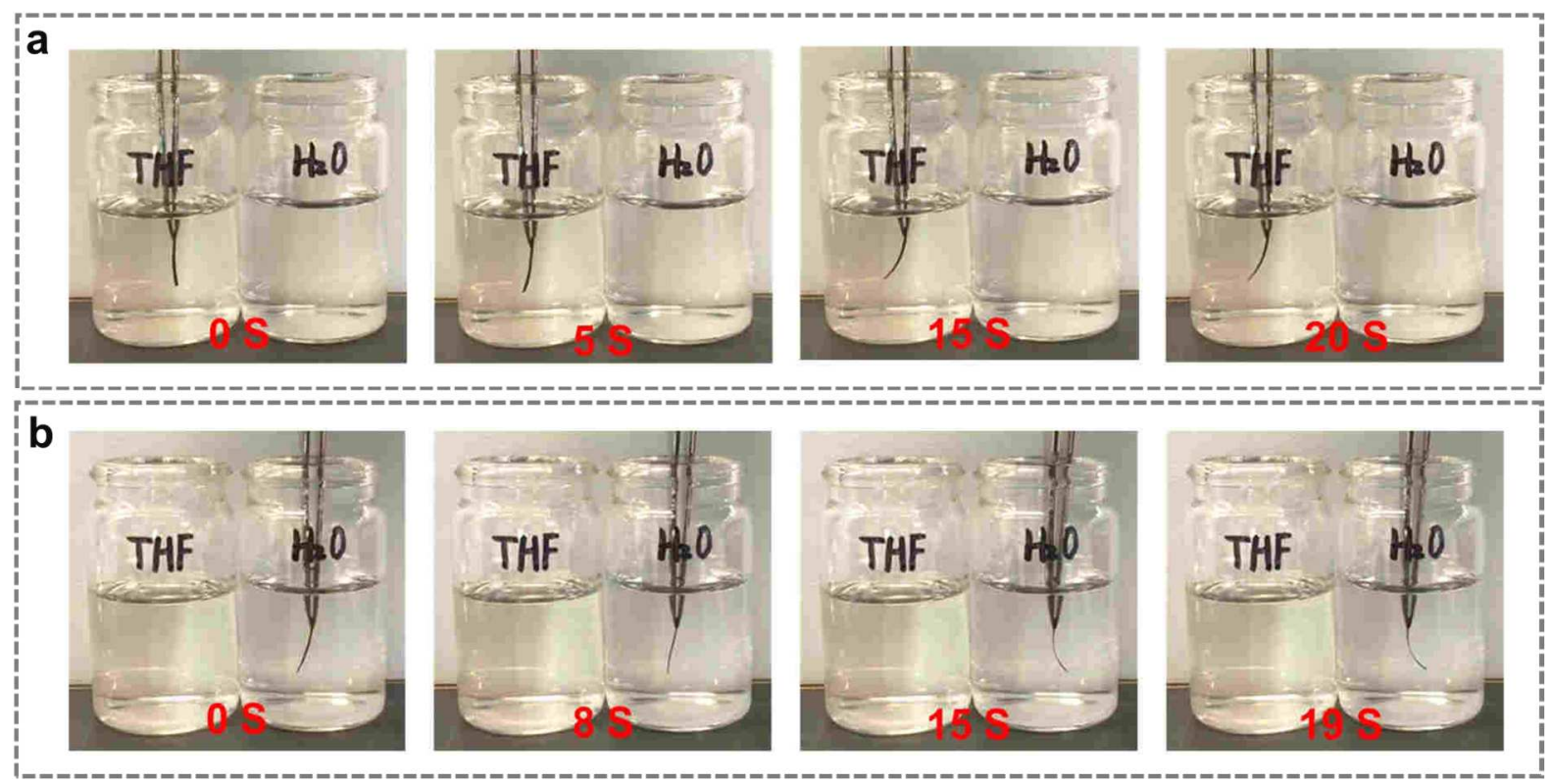

Figure S19. Responsiveness of the Janus-like film of CNWs-SU-C18/75+GO(2:1). a) Photos showing bending motions of the Janus-like stripe in THF at $0,5,15$, and $20 \mathrm{~s}$. b) Snapshots of shape recovery within water at $0,8,15$, and $19 \mathrm{~s}$. 


\section{WILEY-VCH}

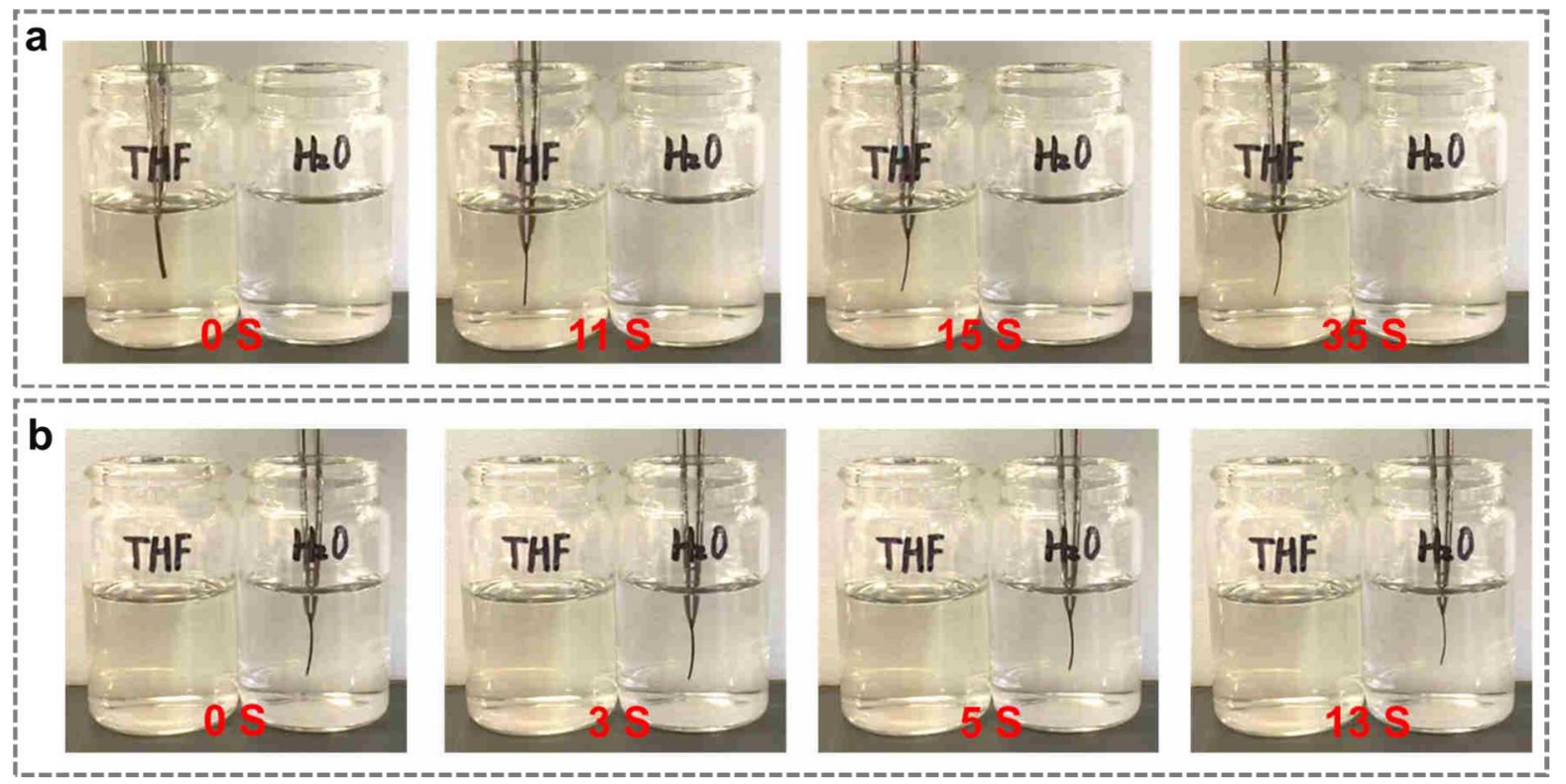

Figure S20. Responsiveness of the Janus-like film of CNWs-SU-C18/75+GO(1:2). a) Photos showing bending motions of the Janus-like stripe in THF at $0,11,15$, and $35 \mathrm{~s}$. b) Snapshots of shape recovery within water at $0,3,5$, and $13 \mathrm{~s}$.

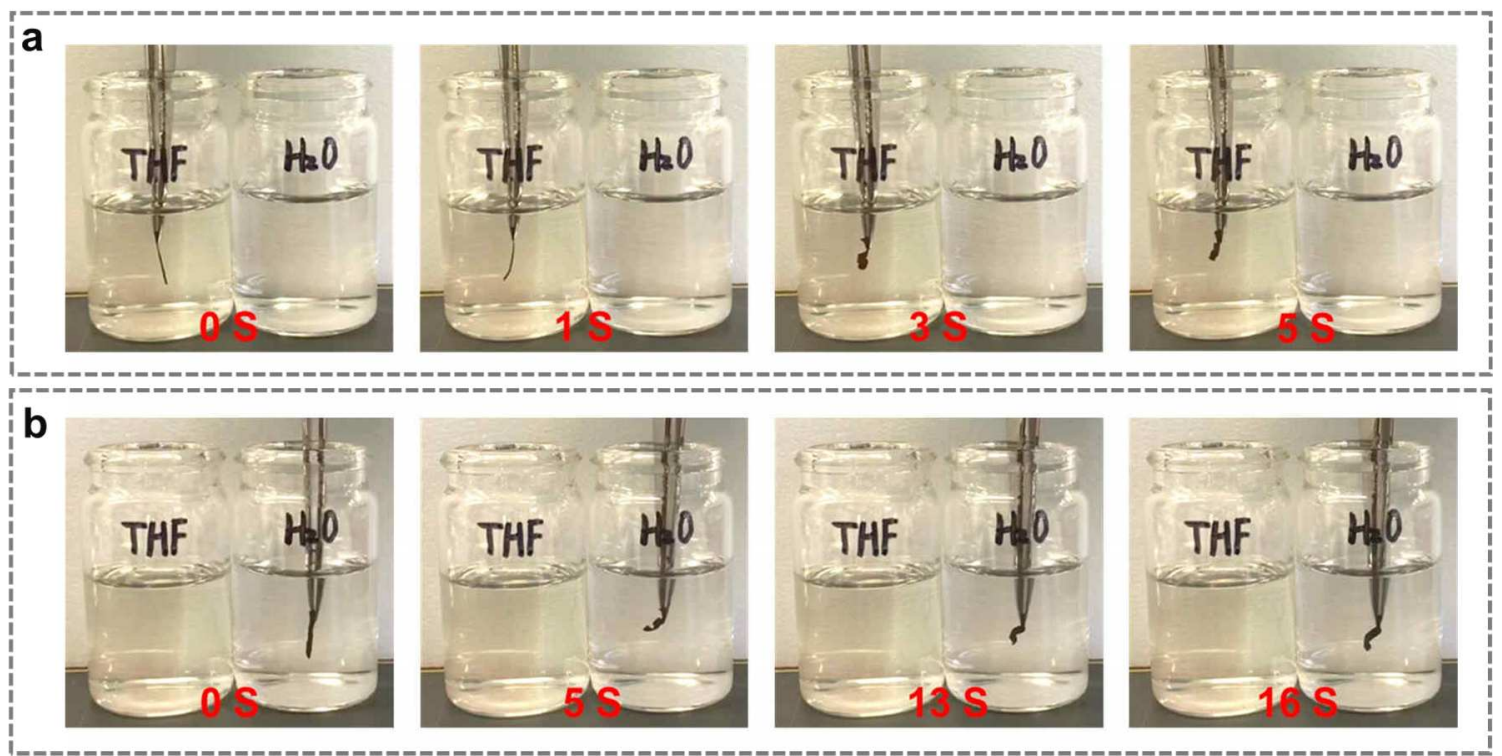

Figure S21. Responsiveness of the Janus-like film of CNWs-SU-C18/75+GO(8:1). a) Photos showing bending motions of the Janus-like stripe in THF at $0,1,3$, and $5 \mathrm{~s}$. b) Snapshots of shape recovery within water at $0,5,13$, and $16 \mathrm{~s}$. 


\section{WILEY-VCH}

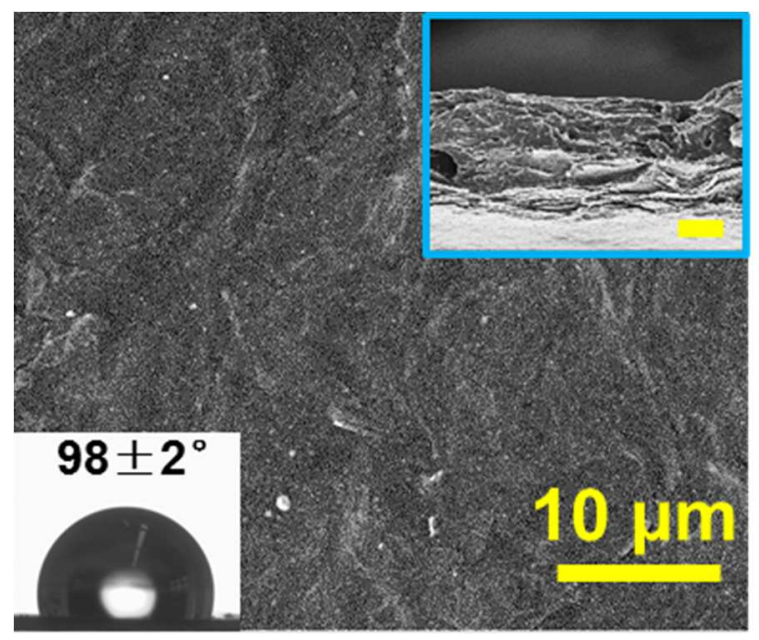

Figure S22. SEM image of the bottom surface of CNWs-SU-C18/75+GO(4:1) Janus-like film after the exposure in THF. The top right inset shows the cross section of the Janus-like film. The scale bar is $10 \mu \mathrm{m}$. The bottom left inset shows static water contact angle on the bottom surface.

\section{Reference}

1. E. Lizundia, A. Urruchi, J.L. Vilas, L.M. León, Carbohydr. Polym. 2016, 136, 250.

2. L. Liu , Y. Gao, Q. Liu , J. Kuang, D. Zhou, S. Ju, B. Han , Z. Zhang, Small 2013, 9, 2466. 


\section{WILEY-VCH}

\section{Caption for Supplementary Movies}

\section{Movie S1}

The transparency change on a film of CNWs-SU-C18/75 after the treatment at $60{ }^{\circ} \mathrm{C}$ and cooling to room temperature.

\section{Movie S2}

The reversible bending/unbending deformation of CNWs-SU-C18/75+GO(4:1) film placed in THF and water under ambient conditions.

\section{Movie S3}

The reversible bending/unbending deformation of CNWs-SU-C18/75+GO(4:1) film placed in THF and water under ambient conditions for 25 runs.

\section{Movie S4}

The reversible bending/unbending deformation of CNWs-SU-C18/75+GO(4:1) film placed in THF-water mixture (the volume ratio of 3 to 1 ) and water under ambient conditions.

\section{Movie S5}

The reversible bending/unbending deformation of CNWs-SU-C18/75+GO(2:1) film placed in THF and water under ambient conditions.

\section{Movie S6}

The reversible bending/unbending deformation of CNWs-SU-C18/75+GO(1:2) film placed in THF and water under ambient conditions.

\section{Movie S7}

The reversible bending/unbending deformation of CNWs-SU-C18/75+GO(8:1) film placed in THF and water under ambient conditions. 


\section{Publication 3}

Self-assembly of surface-acylated cellulose nanowhiskers confined in honeycomb films

Huan Liu, Bo Pang, Kai Zhang*

Author affiliations

*Corresponding author

H. Liu, B. Pang, K. Zhang*

Dept. Wood Technology and Wood-based Composites, University of Goettingen, Büsgenweg

4, Göttingen 37077, Germany

*E-mail: kai.zhang@uni-goettingen.de

Originally submitted to:

ACS Sustainable Chemistry \& Engineering 2020 
Abstract: The self-assembly of cellulose nanowhiskers (CNWs) in confined geometries provides a powerful method to break the symmetry of an ordered structure, thus it can serve as an efficient route for fabrication novel morphologies. Herein, three-dimensional (3D) ordered microporous CNWs honeycomb films were prepared by breath figure (BF) method. The resulting films show high porous order over large regions and the iridescent color was only displayed by the rim of the obtained honeycomb film due to the confined 3D geometry drastically affects the self-assembly of CNWs, which is different than traditional dish-cast CNWs films showing the iridescent color over the whole area. In the confined 3D walls, CNWs self-assemble into cholesteric architectures which resulted in the iridescent color of the rims. Some critical influencing factors, such as the concentration of the CNWs solution and the relative humidity of the atmosphere were also investigated. Too low or too high concentrations of CNWs solutions/relative humidity are not preferable for the formation of uniform porous films and the self-assembly of CNWs in the confined geometry was also affected. This method provides a new idea to study the interactions of CNWs beyond what has been achieved in a planar geometry.

Keywords: surface-acylated cellulose nanowhiskers, self-assembly, breath figure, honeycomb film

\section{Introduction}

The self-assembly of cellulose nanowhiskers (CNWs) has attracted intensive interest in the nanoscience and materials science community, owing to their ability to form chiral nematic crystals in aqueous suspensions. ${ }^{[1]}$ When CNWs adopt a chiral nematic organization, the origination of the CNWs rotates in layers with a characteristic repeating distance. ${ }^{[2]}$ Following the evaporation of water, CNWs retain their chiral nematic order. Traditionally, the self-assembly of CNWs has been studied in planar geometries, which can be defined as onedimensional (1D) confined system. This is the simplest and most studied case of CNWs in confinement, in which CNWs are placed on the substrate with the other surface being in direct contact with the atmosphere. Thin iridescent films with many applications, such as photonic sensors, filters, and photoelectronic devices, were obtained in this case. ${ }^{[3]}$

More recently, there has been increasing study into the self-assembly of CNWs in twodimensional (2D) or three-dimensional (3D) confined geometries since the confinement effect can effectively break the symmetry of a structure and lead to strong deviation from an equilibrium morphology. Compared to self-assembly of CNWs in bulk, self-assembly of 
CNWs in constrained geometries such as narrow channels or spherical confinement result in novel structures with potentially useful properties. ${ }^{[4]}$ Confinement of CNWs is realized by placing them inside a confining environment. For the 2D confinement, Liu et al. reported the self-assembly of CNWs in a continuous cylindrical geometry to fabricate hierarchical liquid metacrystal fibers. ${ }^{[5]}$ The resulted fibers exhibit polarized light-direction and -intensity controllability. Fully enclosed spherical geometry, as a kind of 3D confinement, has been applied to the self-assembly of CNWs. ${ }^{[4 \mathrm{~d}, 4 \mathrm{e}]}$ Uniform spheres with chiral nematic structures from CNWs can be fabricated with this method. Although these confined geometries can constrain the self-assembly of CNWs into chiral nematic structures, they are all static processes in which CNWs are confined from the beginning.

In this study, we investigate the self-assembly of CNWs confined in 3D honeycomb films which fabricated through breath figure (BF) method. The resulting films show high porous order over large regions. Moreover, the rim of the obtained honeycomb film displayed iridescent color due to the self-assembly of CNWs into chiral nematic architectures in the confined 3D walls of pores which generated by the gradually condense of water droplets. To the best of our knowledge, this is the first report of constrained self-assembly of CNWs in dynamic 3D geometry and it is the first report of honeycomb-like microporous films with iridescent rim induced by the BF method using CNWs.

\section{Results and discussion}



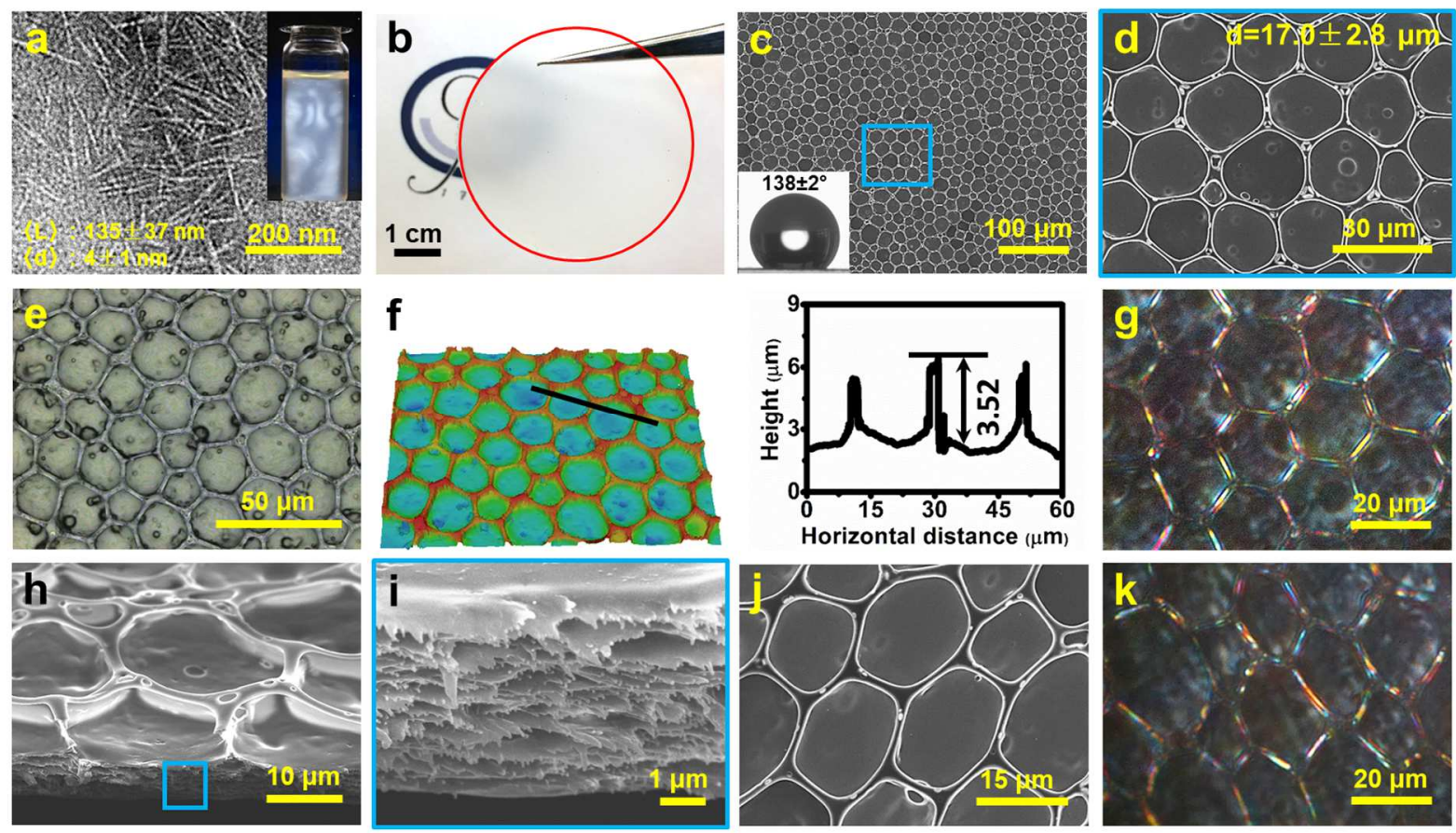

Figure 1. a) A representative TEM image of CNWs-SU. The inset shows DCM suspension of CNWs-SU (10 mg/mL) between crossed polarizers. b) Photo image of the dried honeycomb film fabricated with CNWs-SU. c) Representative SEM image of the top surface of the honeycomb film fabricated with CNWs-SU. The inset shows static water contact angle (SWCA) on the top surface. d) SEM image of the magnified region as in (c). e) Representative laser scanning microscope (LSM) image of the honeycomb film fabricated with CNWs-SU. f) 3D LSM image and height profile of the honeycomb film fabricated with CNWs-SU. g) Polarized light microscopy (PLM) image of the honeycomb film fabricated with CNWs-SU. h) Representative SEM image of the cross section of the honeycomb film fabricated with CNWs-SU. i) SEM image of the magnified region as in (h). j) Representative SEM image the dried honeycomb film fabricated with CNWs-SU after stretching. k) PLM image the dried honeycomb film fabricated with CNWs-SU after stretching.

CNWs with surface-immobilized 10-undecenoyl groups (CNWs-SU) were synthesized from microcrystalline cellulose (MCC) after the surface esterification by 10-undecenoyl chloride (Figure S1). The yield was about 10\% and the degree of substitution (DS) of CNWsSU was measured to be 1.32 based on the elemental analysis. The obtained CNWs-SU have an average length of $135 \pm 37 \mathrm{~nm}$ and an average diameter of $4 \pm 1 \mathrm{~nm}$ based on the measurements on 100 single CNWs (Figure 1a). Based on the signals ascribed to crystalline 
(88.5 ppm) and amorphous (83.7 ppm) domains, the crystallinities of MCC and CNWs-SU are determined to be about $58 \%$ and $40 \%$, respectively (Figure S2).

CNWs-SU honeycomb films were prepared using a static BF approach in water saturated atmosphere (Figure S3). $10 \mathrm{~mL}$ of a DCM solution of CNWs-SU (3 mg/mL) was cast on Teflon mould and kept at room temperature with a relative humidity of $75 \%$. After complete evaporation of solvent and water droplets, we obtained honeycomb films with microscale pores on the top surfaces (Figure $1 \mathrm{~b}$ and $\mathrm{c}$ ). The porous surfaces gave rise to high contact angle due to their structured surfaces. Flat surface possessed a hydrophilic character with an average SWCA of $86 \pm 3^{\circ}$ (Figure S4), while the porous structured surface increased the SWCA into the hydrophobic region of about $138 \pm 2^{\circ}$ (Figure 1c). The SEM image shows that the diameter of pores was around $17.0 \pm 2.8 \mu \mathrm{m}$ and the width of the rims was about $1.8 \pm 0.4$ $\mu \mathrm{m}$ (Figure 1d). The LSM measurement also reveals that the obtained film exhibited honeycomb-like structures (Figure 1e). Microsized pores uniformly distributed on the top surface of the films. According to the height profile obtained from 3D LSM image, the average depth of the pores was about $3.7 \pm 0.5 \mu \mathrm{m}$ (Figure 1f). While just few small pores distributed on the bottom surface of the honeycomb film (Figure S5). It is worth pointing out that the rim of the obtained honeycomb film displayed iridescent color as shown in the PLM image (Figure 1g). Ring-shaped iridescent patterns were observed around the pores, but no iridescent color was observed from the interior part of the pore, indicating that the CNWs highly arranged along the boundary of micropores. Furthermore, normal CNWs-SU film was used as the control and did not show any iridescence at all (Figure S4). The SEM crosssectional view in Figure 1h shows the contour of the pores. The depth of the pores was about $3.8 \mu \mathrm{m}$ which consistent with the result of 3D LSM. The height of the underneath diameter was about $10.3 \pm 0.5 \mu \mathrm{m}$. The pore depth and underneath diameter were smaller than the open diameter $(17.0 \pm 2.8 \mu \mathrm{m})$ of the pore in the top surface. Accordingly, the aspect ratio of depth of pores and the height of underneath diameter $\left(\mathrm{r}_{\mathrm{D} / \mathrm{H}}\right)$ was about $35.6 \%$. From the magnified SEM image of the cross section, the CNW film showed typical layered morphology (Figure 1i).

Stretching is a simple secondary processing method for mechanical transformation of the pore shape and size of honeycomb-patterned films. With the help of applied tensile force, the micropores elongated in the direction parallel to the force, while they compressed in the perpendicular direction. Thus, the surface micropores turned from original circular shape into rectangular shape (Figure $1 \mathrm{j}$ and Figure S6). The sides of the rectangular pores were elongated 
along the stretching direction, and the other sides were decreased in length. The ring-shaped iridescent pattern was again observed, as shown in Figure 1k, indicating that the stretching has no influence on the arrangement of CNWs after drying as a dried film.

HC films have unique double-layered structures supported by pillars. After peeling off the top layer of the film using adhesive tape, a pincushion structure can be formed (Figure S7a and $\mathrm{b}$ ). The obtained pinned topological features significantly escalate the SWCA to an average value of $144 \pm 3^{\circ}$. This is strongly coupled to the apparent surface area that is smaller than the normal close pore structure and results in lower wettability of the surface due to the formation of air pockets under the water droplet. ${ }^{[6]}$
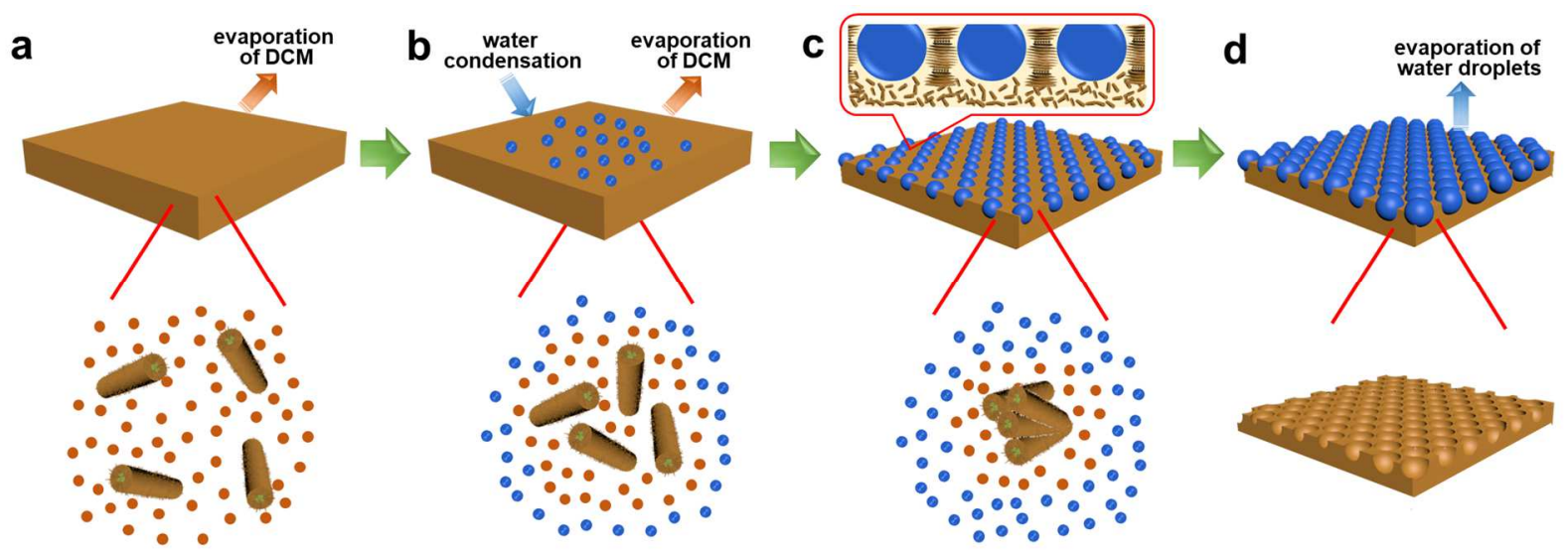

Figure 2. Schematic illustration for the self-assembly process of CNWs-SU into honeycomb films.

Figure 2 presents a schematic explanation as to how the ordered porous films form. At first, the CNWs solution spread on the substrate homogeneously (Figure 2a). As the solvent evaporates, leading to the cooling of the solution surface, the surrounding water vapor forms small droplets and deposits on the surface of the solution. The presence of water droplets changes the characteristics of the solvent dramatically: inducing a phase inversion and aggregation of the hydrophobic CNWs (Figure $2 b$ ). The continuous condensation of water vapor, causes the growth and self-organization of the water droplets into ordered droplet array and forces the CNWs to move closer together to form aggregates (Figure 2c), thereby induces the formation of walls and pores. The walls, composed of stacked and overlapped CNWs, are 
highly arranged along the boundary of micropores. Complete evaporation of DCM and then the water droplets, the ordered porous films formed (Figure 2d).
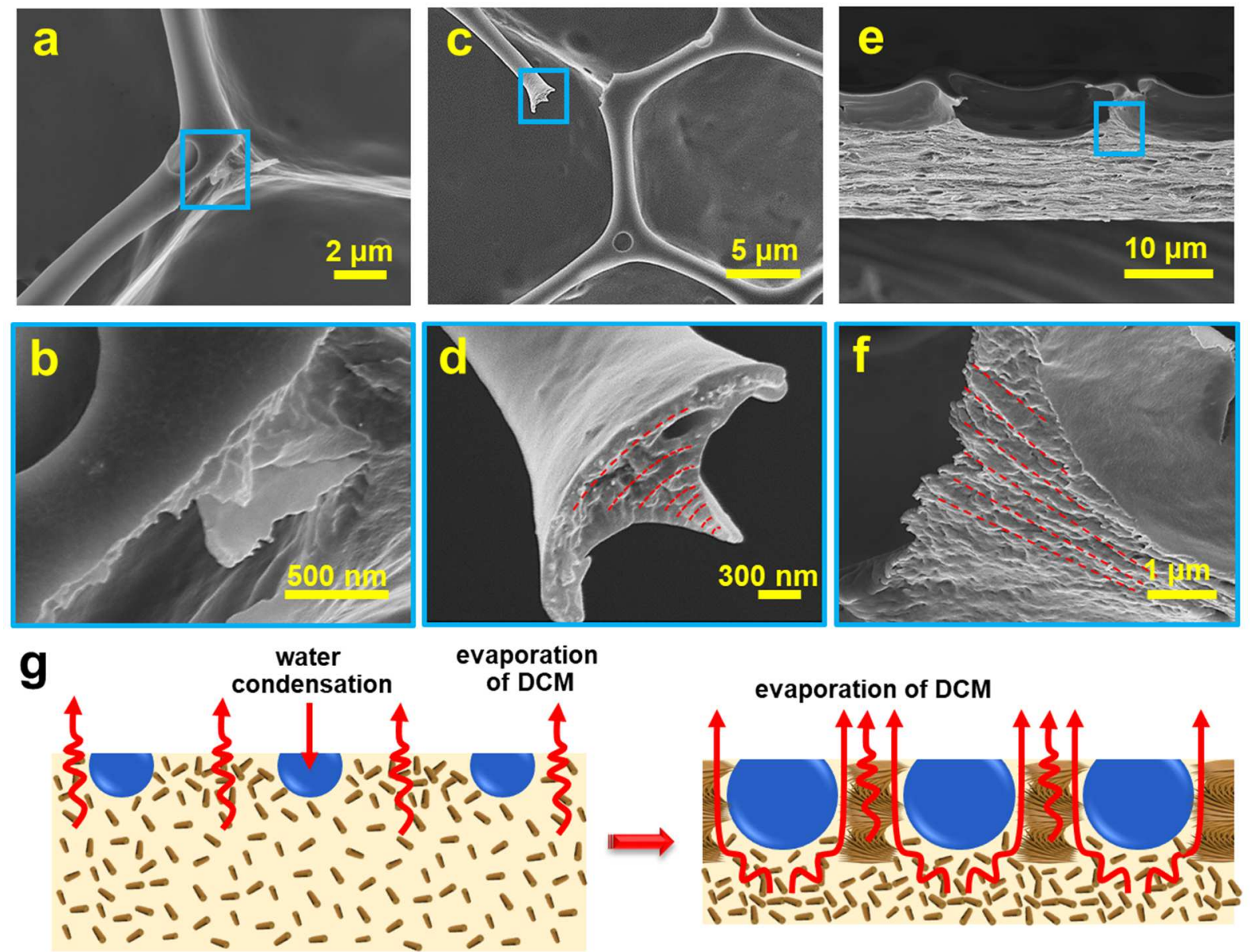

Figure3. (a) and (c) Representative SEM image of the honeycomb film fabricated with CNWs-SU after removing part of walls of the pores. (b) and (d) SEM images of the magnified region as in (a) and (c), respectively. e) Representative SEM image of the cross section of the honeycomb film fabricated with CNWs-SU. f) SEM image of the magnified region as in (h). g) Schematic description for the formation of iridescent rim.

To understand the mechanism of the self-assembly of CNWs-SU into honeycomb films with iridescent rims, we detected the structures of the walls of pores. Figure $3 \mathrm{a}$ shows the top surface of honeycomb film after removing part of walls of the pores with a top view. The magnified SEM image clearly reveals the lamellar-like structure of the walls of pores (Figure 3b). From another SEM image of the damaged pores, we can see the cross section of the walls 
of pores present ginkgo-leaf-like structure (Figure 3c,d). Figure 3d shows the lamellar-like structures where CNWs-SU are organized into a chiral nematic structure with a periodic spacing of about $300 \mathrm{~nm}$. For the underside of the walls of pores, we can see the slandered lamellar structure. This is mainly due to the width of the wall is getting narrower from bottom to top. With the growth of water droplets, CNWs-SU were squeezed and formed the slanted lamellar structure. We also proposed the mechanism for iridescent rim formation in honeycomb film during casting (Figure 3g). The CNWs-SU concentration was uniform in the initial aqueous suspension. However, as the water droplets formed on the surface of the suspension, the DCM will evaporate along with the areas where no water droplets formed. We predicted that two distinct regions would arise, one under the water droplets, where evaporation is restricted, and another outside the water droplets, where evaporation can occur. In the confined 3D walls, CNWs self-assembly into cholesteric architectures which resulted in the iridescent color of the rims. The arrangement presumably occurs upon the growth of the aqueous droplets during the BF process. The stage of self-assembly is very important in determining the resulting film's optical properties.
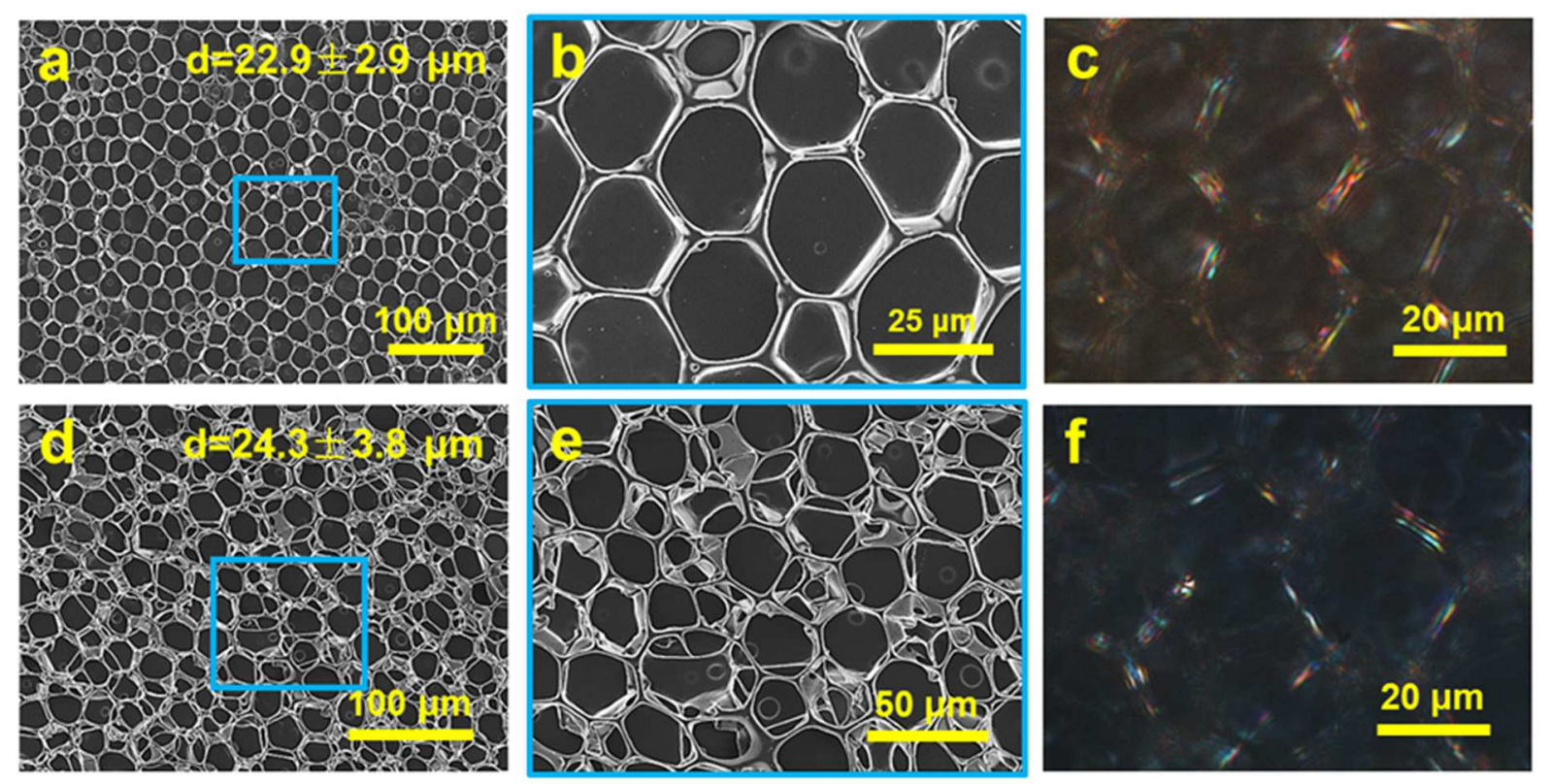

Figure 4. a) Representative SEM image of the top surface of the honeycomb film fabricated with CNWs-SU at the concentration of $7 \mathrm{mg} / \mathrm{mL}$. b) SEM image of the magnified region as in (a). c) PLM image of the top surface of the honeycomb film fabricated with CNWs-SU at the concentration of $7 \mathrm{mg} / \mathrm{mL}$. d) Representative SEM image of the top surface of the honeycomb film fabricated with CNWs-SU at 96\% relative humidity. e) SEM image of the magnified 
region as in (d). f) PLM image of the top surface of the honeycomb film fabricated with CNWs-SU at $96 \%$ relative humidity.

The effects of solution concentration and relativity humidity on the morphologies of the resulting films were also investigated in this study.

The pore size and regularity of BF arrays are sensitive to the concentration of the solution. As a result, each CNWs solution with a different concentration yielded an entirely different morphology. However, when the concentration of CNWs was increased to $7 \mathrm{mg} / \mathrm{mL}$, the resulting films exhibited pores with irregular shapes. The average pore size of the film was measured at $22.9 \pm 2.9 \mu \mathrm{m}$ (Figure 4a,b) and the depth of pore increased to $7.5 \pm 1.0 \mu \mathrm{m}$ according to the result of LSM (Figure S8). It is evident that the concentration increase facilitates the formation of deep holes. The height of the underneath diameter was about $18.0 \pm 0.7 \mu \mathrm{m}$. Accordingly, the $\mathrm{r}_{\mathrm{D} / \mathrm{H}}$ was about $41.7 \%$, which was higher than the honeycomb film fabricated at the concentration of $3 \mathrm{mg} / \mathrm{mL}$. A malformed morphology was obtained when the concentration of CNWs was very high (Figure S9a). The increased viscosity will weaken the convection in the solution, which is not beneficial to the self-assembly of droplets, and the pores will become disordered when the concentration is very high. ${ }^{[7]}$ On the other hand, the high concentration resulted in the poor self-assembly of CNWs in this kind of disorder geometry which shows in the not obvious iridescent color of the rim (Figure 4c). Honeycomb structures were not observed at the CNWs concentration at $3 \mathrm{mg} / \mathrm{mL}$ and the volume of $5 \mathrm{~mL}$ (Figure S9b). This result implies that an adequate amount of CNWs is required to form the honeycomb structures. This maybe stems from inefficient encapsulation and stabilization of the condensed water droplets levitation on the interface due to too low quantity of CNWs.

As the humidity of the atmosphere increased from $75 \%$ to $96 \%$, the average pore size systematically increased from $17.0 \pm 2.8 \mu \mathrm{m}$ to $24.3 \pm 3.8 \mu \mathrm{m}$ (Figure $4 \mathrm{~d}, \mathrm{e}$ ). The high humidity results in the coalescence of water droplets, yielding a polydisperse pore size distribution. The depth of pores increased from $3.7 \pm 0.5 \mu \mathrm{m}$ to $10.6 \pm 2.2 \mu \mathrm{m}$ when the relative humidity changed from $75 \%$ to $96 \%$ (Figure S10), while the height of the underneath diameter almost unchanged. Thus, the $\mathrm{r}_{\mathrm{D} / \mathrm{H}}$ was about $98.1 \%$, which was much higher than the honeycomb film fabricated at the relative humidity of $75 \%$. The higher humidity not only forms larger water droplets but also slows the solvent evaporation, both of which lead to the growth of droplets 
and the formation of larger pores. ${ }^{[8]}$ When the relative humidity was $60 \%$, micropores uneven distributed on the top surface and even some areas did not form micropores (Figure S11). The average pore size was about $12.9 \pm 1.7 \mu \mathrm{m}$ and the depth of pores was about $3.2 \pm 0.9 \mu \mathrm{m}$ (Figure S12). In the relative humidity was below $50 \%$, only a flat film was obtained without regular arrangement of holes due to the failure condensation of water (Figure S13). The humidity of the atmosphere influences the evaporation of the CNWs solvents, which further affect the self-assembly of CNWs in the confined 3D geometry (Figure 4f).

\section{Conclusion}

In summary, a porous CNWs-SU film with well-ordered porous structure was prepared by using the breath figure method. The rim of the obtained honeycomb film displayed iridescent color due to the confined 3D geometry drastically affects the self-assembly of CNWs. The rims, composed of stacked and overlapped CNWs, are highly arranged along the boundary of micropores. The arrangement presumably occurs upon the growth of the aqueous droplets during the breath-figure process. Additionally, some critical influencing factors, such as the concentration of the CNWs solution and the relative humidity of the atmosphere were also investigated. This simple technique unlocks new possibilities in designing the visual appearance of such iridescent films. We expect these porous films with structural color may lead to a broad application of sophisticated optical materials.

\section{Experimental Section}

Materials: Microcrystalline cellulose (MCC) (Avicel PH101) with an average particle size of about $50 \mu \mathrm{m}$, and 10-undecenoyl chloride were purchased from Sigma-Aldrich Chemie GmbH (Steinheim, Germany). Dry pyridine, tetrahydrofuran (THF) and dichloromethane (DCM) were brought from Th. Geyer GmbH \& Co. KG (Renningen, Germany). All chemicals are all analytical grade and used as received. Deionized water (DI water) was used in all experiments.

Preparation of surface-acylated cellulose nanowhiskers (CNWs-SU): CNWs-SU were prepared according to our previous work with a few modifications. ${ }^{[9]}$ Typically, $1 \mathrm{~g}$ of MCC and $30 \mathrm{~mL}$ of pyridine were fed into a three-necked flask. The reaction mixture was stirred and heated up to $50{ }^{\circ} \mathrm{C}$. Then, 10 -undecenoyl chloride $(2.73 \mathrm{~mL}, 2 \mathrm{~mol}$ acid chloride per mol anhydroglucose units of cellulose) was added to the cellulose suspension under stirring. After $7 \mathrm{~h}$ stirring at $50{ }^{\circ} \mathrm{C}$, the reaction mixture was poured into $200 \mathrm{~mL}$ methanol immediately. The precipitate was separated by centrifugation. Thereafter, the product was purified by a repeated 
dispersion and precipitation process using THF and methanol, respectively, before the product was dispersed in THF. Then, the suspension was centrifuged for $10 \mathrm{~h}$ at $14000 \mathrm{rpm}$ at $4{ }^{\circ} \mathrm{C}$. After the centrifugation, the supernatant was removed and the solid was dispersed in THF again, which was centrifuged for $20 \mathrm{~min}$ at $3000 \mathrm{rpm}$ at $20{ }^{\circ} \mathrm{C}$ to remove the micro-scaled segments. Finally, the purified product was dispersed in DCM for further use. The corresponding yield of CNWs-SU was $11.6 \pm 2.4 \%$. The DS of the obtained CNWs-SU was determined to be 1.32 .

Preparation of honeycomb films from CNWs-SU: Honeycomb films with controlled microporous structures were fabricated via breath figure method, as illustrated in Figure S3. In a typical process, CNWs-SU were first dissolved in DCM to a certain concentration, and then $10 \mathrm{~mL}$ of the CNWs-SU solution was cast onto the Teflon mould in the desiccator at different relative humidity. The saturated relative humidity in the chamber was fixed by saturated salt solutions, i.e., $44 \%\left(\mathrm{~K}_{2} \mathrm{CO}_{3}\right), 57 \%(\mathrm{NaBr}), 75 \%(\mathrm{NaCl})$, and $96 \%\left(\mathrm{~K}_{2} \mathrm{SO}_{4}\right){ }^{[10]}$ The temperature was kept at room temperature during the whole process. After the complete evaporation of solvent as well as the water droplets that spontaneously condensed at the airsolution interface, honeycomb films with controlled porous structures were obtained on the surface of the Teflon substrate.

Transmission electron microscopy (TEM): TEM images of the CNWs-SU were acquired with a CM 12 Transmission Electron Microscope (Philips, Netherland). The specimen was stained by phosphotungstic acid solution $(0.2 \mathrm{wt} \%$ in THF) for about $2 \mathrm{~min}$ to enhance the contrast.

Scanning electron microscopy (SEM): SEM images were captures by a LEO Supra-35 highresolution field emission scanning electron microscope (Carl Zeiss AG, Germany) at an accelerating voltage of $5 \mathrm{kV}$. A layer of carbon was coated on the surface of samples before SEM measurements.

Polarized light micrographs (PLM): PLM were obtained through Nikon ECLIPSE E600 imaging microscope with the polarizers in a perpendicular arrangement and the camera type of Nikon DS-Fi2.

Laser scanning microscope (LSM): LSM images were obtained on a VK-X100K 3D laser scanning microscope (Keyence Corporation, Germany) under ambient conditions. 
Elemental analysis: The contents of carbon, hydrogen and sulfur were determined with an Elemental Analyser 4.1 vario EL III (Elementar, Germany). DS of corresponding groups was calculated according to the carbon contents, as the following formula: ${ }^{[11]}$

$$
\mathrm{DS}=\frac{5.13766-11.5592 \times C \%}{0.996863 \times C \%-0.856277 \times n+n \times C \%}
$$

where $\mathrm{C} \%$ is the content of elemental carbon and $\mathrm{n}$ is the number of carbon atoms in the acyl moieties, respectively.

Solid-state ${ }^{13}$ C CP-MAS NMR spectroscopy: Solid-state ${ }^{13} \mathrm{C}$ CP-MAS NMR measurements for all samples were acquired at ambient conditions using a Bruker AVANCE IIIHD 800WB spectrometer with a field strength of $18.8 \mathrm{~T}$. Experiments were performed using a Bruker 1.3 mm MAS triple resonance probe with a MAS spinning frequency of $55.555 \mathrm{kHz}$. A ramped CP-MAS sequence was used with a ${ }^{1} \mathrm{H}$ excitation power of $138.8 \mathrm{kHz}$ and a Hartman Hahn contact time of $2 \mathrm{~ms}$ with a ${ }^{13} \mathrm{C}$ inherent radio frequency (rf) field strength of $62.5 \mathrm{kHz}$ and ${ }^{1} \mathrm{H}$ rf field strength of $127.4 \mathrm{kHz}$. During the acquisition of $27 \mathrm{~ms}$, a ${ }^{1} \mathrm{H}$ decoupling was performed with an inherent rf field of $14.7 \mathrm{kHz}$ employing WALTZ-16 sequence. ${ }^{[12]}$ In a typical $8 \mathrm{~h}$ experiment, 15360 scans were accumulated with a recycle delay of $2.5 \mathrm{~s}$.

The crystallinities of the samples were determined based on the solid-state ${ }^{13} \mathrm{C} \mathrm{CP-MAS}$ NMR spectra. The individual peaks ascribed to $\mathrm{C} 4$ in crystalline and amorphous regions were extracted by a peak deconvolution process in Origin 8.5 using Gaussian function (Supplementary Figure 1). The crystallinity was calculated using the following equation: ${ }^{[13]}$

$$
\text { Crystallinity }(\%)=\frac{A_{c r}}{A_{c r .+A_{a m}}}
$$

where Acr. and Aam. represent the areas obtained by the integration of the fitted curves of the crystalline peak and amorphous peak in the $\mathrm{C} 4$ region, respectively.

\section{Acknowledgements}

K.Z. thanks German Research Foundation (DFG) for the financial support for the project (Project Nr. ZH546/2-1) and Georg-August-University of Goettingen for the start-up funding. H.L. and B.P. thank the Chinese Scholarship Council (CSC) for the financial support.

\section{References}

[1] a) Y. Habibi, L. A. Lucia, O. J. Rojas, Chem. Rev. 2010, 110, 3479; b) A. Tran, C. E. Boott, M. J. MacLachlan, Adv. Mater. 2020, 1905876. 
[2] B. Thomas, M. C. Raj, A. K. B, R. M. H, J. Joy, A. Moores, G. L. Drisko, C. Sanchez, Chem. Rev. 2018, 118, 11575.

[3] a) K. Yao, Q. Meng, V. Bulone, Q. Zhou, Adv. Mater. 2017, 29, 1701323; b) T. Hiratani, W. Y. Hamad, M. J. MacLachlan, Adv. Mater. 2017, 29, 1606083; c) S. M. Mahpeykar, Y. Zhao, X. Li, Z. Yang, Q. Xu, Z.-H. Lu, E. H. Sargent, X. Wang, Adv. Opt. Mater. 2017, 5, 1700430; d) M. Giese, M. Spengler, Mol. Syst. Des. Eng. 2019, 4, 29.

[4] a) P. Rofouie, E. Galati, L. Sun, A. S. Helmy, E. Kumacheva, Adv. Funct. Mater. 2019, 29, 1905552; b) S. N. Fernandes, P. L. Almeida, N. Monge, L. E. Aguirre, D. Reis, C. L. P. de Oliveira, A. M. F. Neto, P. Pieranski, M. H. Godinho, Adv. Mater. 2017, 29, 1603560; c) V. Cherpak, V. F. Korolovych, R. Geryak, T. Turiv, D. Nepal, J. Kelly, T. J. Bunning, O. D. Lavrentovich, W. T. Heller, V. V. Tsukruk, Nano Lett. 2018, 18, 6770; d) Y. Li, J. Jun-Yan Suen, E. Prince, E. M. Larin, A. Klinkova, H. Thérien-Aubin, S. Zhu, B. Yang, A. S. Helmy, O. D. Lavrentovich, E. Kumacheva, Nat. Commun. 2016, 7, 12520; e) R. M. Parker, B. FrkaPetesic, G. Guidetti, G. Kamita, G. Consani, C. Abell, S. Vignolini, ACS Nano 2016, 10, 8443. [5] Y. Liu, P. Wu, Adv. Funct. Mater. 2020, 2002193.

[6] S. Mongkhontreerat, M. V. Walter, O. C. J. Andrén, Y. Cai, M. Malkoch, Adv. Funct. Mater. 2015, 25, 4837.

[7] B. Zhao, J. Zhang, X. Wang, C. Li, J. Mater. Chem. 2006, 16, 509.

[8] C. Wang, Y. Mao, D. Wang, Q. Qu, G. Yang, X. Hu, J. Mater. Chem. 2008, 18, 683.

[9] H. Liu, B. Pang, R. Garces, R. Dervisoglu, L. Chen, L. Andreas, K. Zhang, Angew. Chem., Int. Ed. 2018, 57, 16323-16328.

[10] J. F. Young, J. Appl. Chem. 1967, 17, 241.

[11] C. Vaca-Garcia, M. E. Borredon, A. Gaseta, Cellulose 2001, 8, 225.

[12] a) G. Metz, X. L. Wu, S. O. Smith, J. Magn. Reson., Ser. A 1994, 110, 219; b) A. J. Shaka, J. Keeler, R. Freeman, J. Magn. Reson. (1969) 1983, 53, 313.

[13] a) S. Park, D. K. Johnson, C. I. Ishizawa, P. A. Parilla, M. F. Davis, Cellulose 2009, 16, 641; b) R. H. Newman, L. M. Davies, P. J. Harris, Plant Physiol. 1996, 111, 475. 


\section{Publication 3}

(Supporting Information)

Self-assembly of surface-acylated cellulose nanowhiskers confined in honeycomb films

Huan Liu, Bo Pang, Kai Zhang*

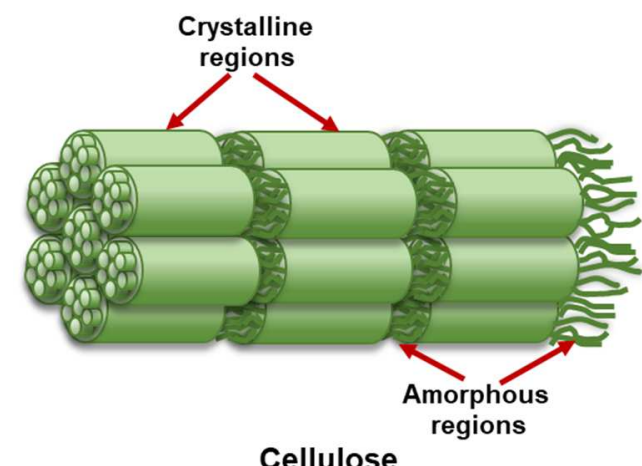

Esterification
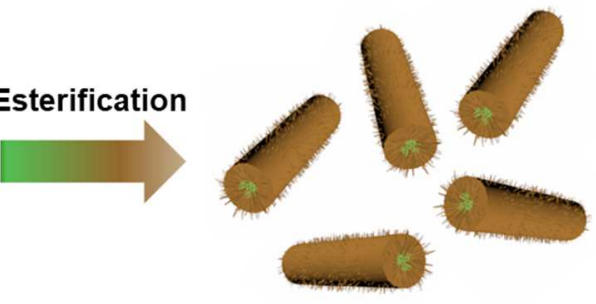

CNWs-SU

Figure S1. Schematic illustration for the synthesis of CNWs-SU.

a

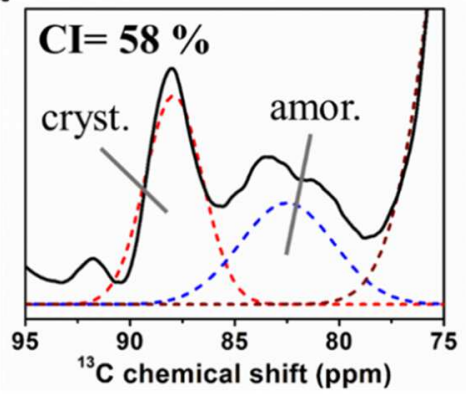

b

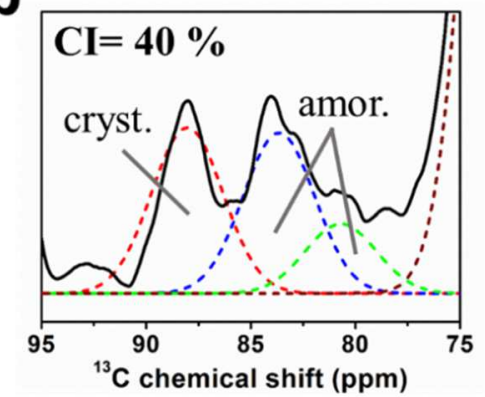

Figure S2. Peak deconvolution for the $\mathrm{C} 4$ region of solid-state $\mathrm{CP}-\mathrm{MAS}{ }^{13} \mathrm{C}$ NMR spectra of a) MCC and b) CNWs-SU.

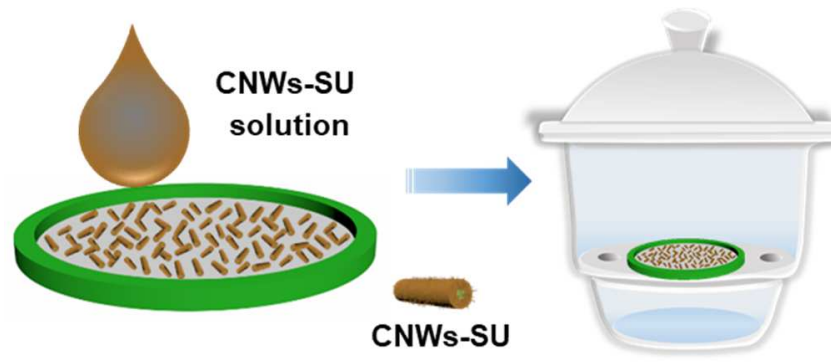

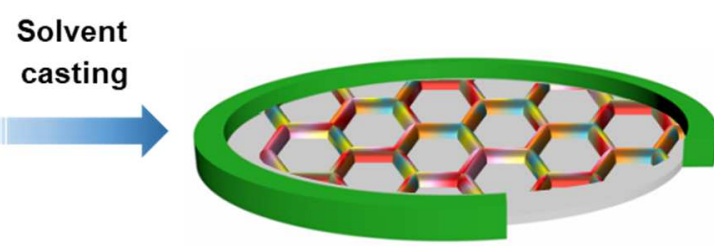

Honeycomb film 
Figure S3. Schematic illustration for the fabrication of CNWs-SU honeycomb film with breath figure method.
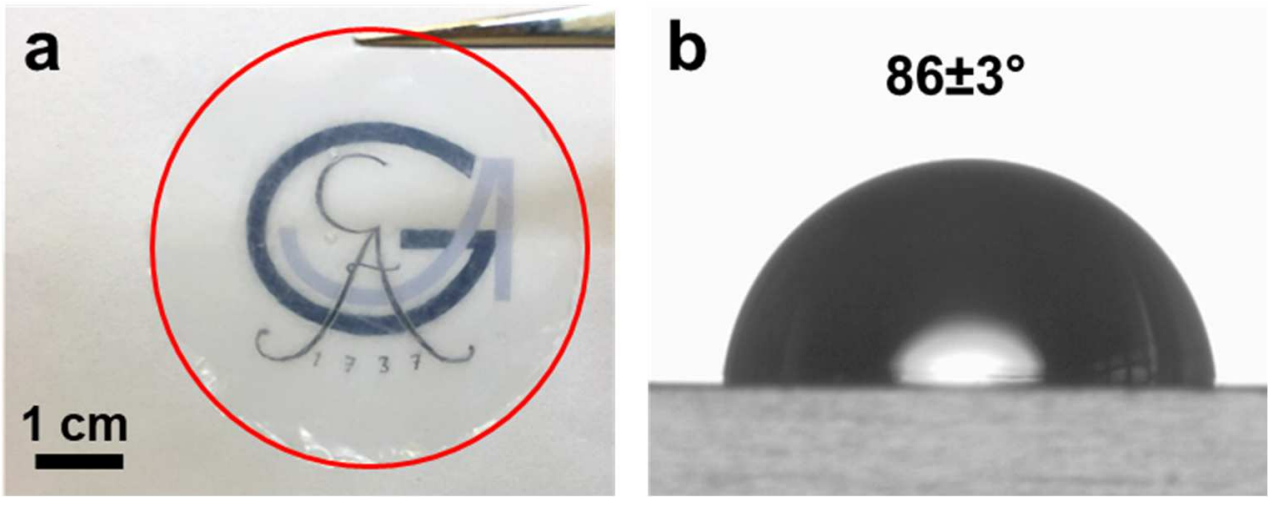

Figure S4. a) Photo image of the dried film fabricated with CNWs-SU. b) Static water contact angle (SWCA) of the dried film fabricated with CNWs-SU.
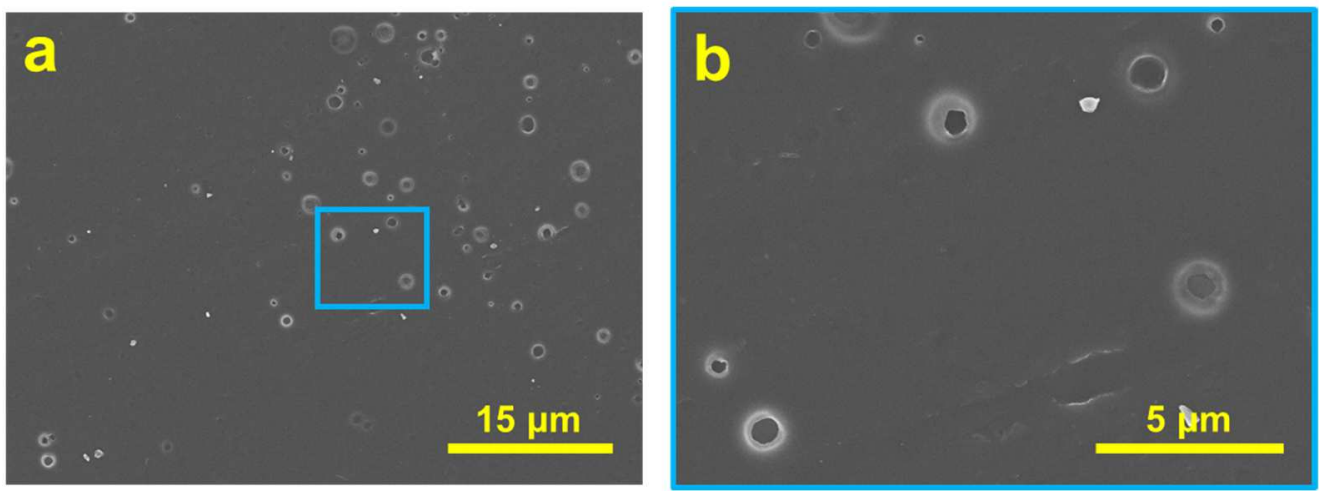

Figure S5. SEM image of the bottom side of the honeycomb film fabricated with CNWs-SU.
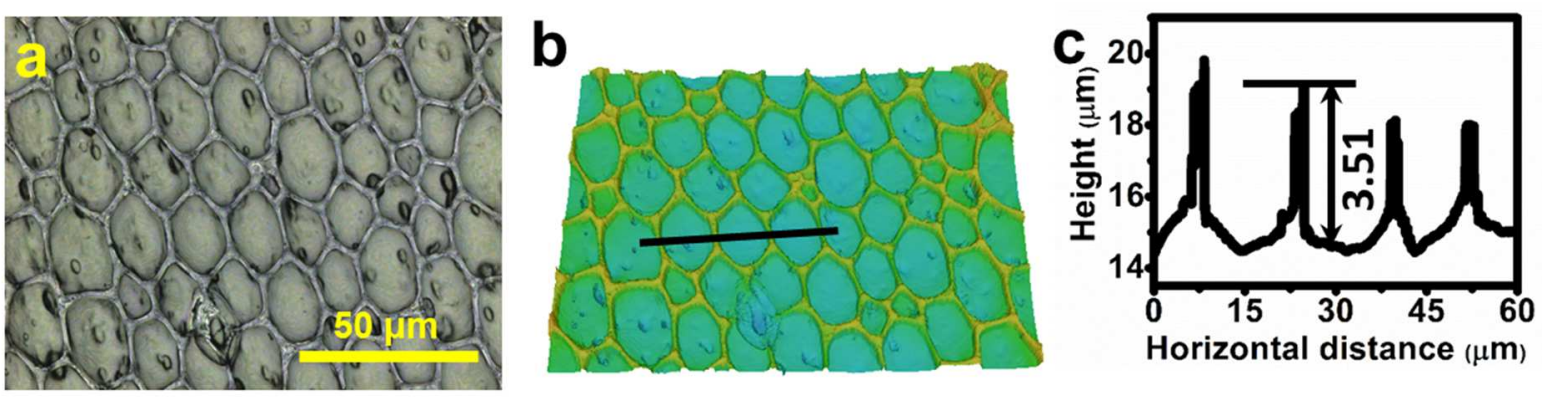
Figure S6. (a) Representative LSM image, (b) 3D LSM image, and (c) height profile of the honeycomb film fabricated with CNWs-SU after stretching.
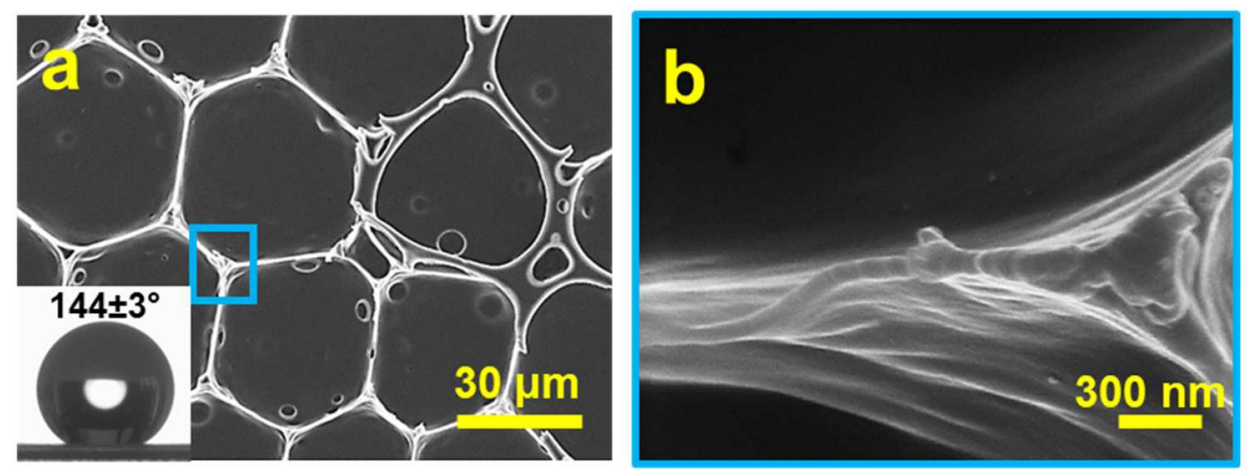

Figure S7. a) Representative SEM image of the top surface of the honeycomb film fabricated with CNWs-SU after peeling off the top layer. The inset shows SWCA after peeling off the top layer. b) SEM image of the magnified region as in (a).
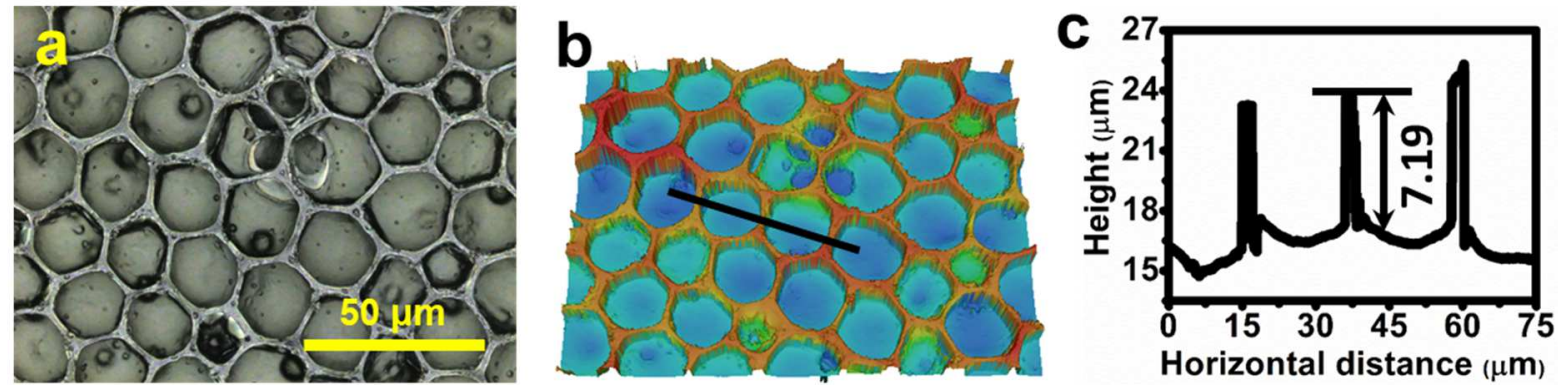

Figure S8. (a) LSM image, (b) 3D LSM image, and (c) height profile of the honeycomb film fabricated with CNWs-SU at the concentration of $7 \mathrm{mg} / \mathrm{mL}$.
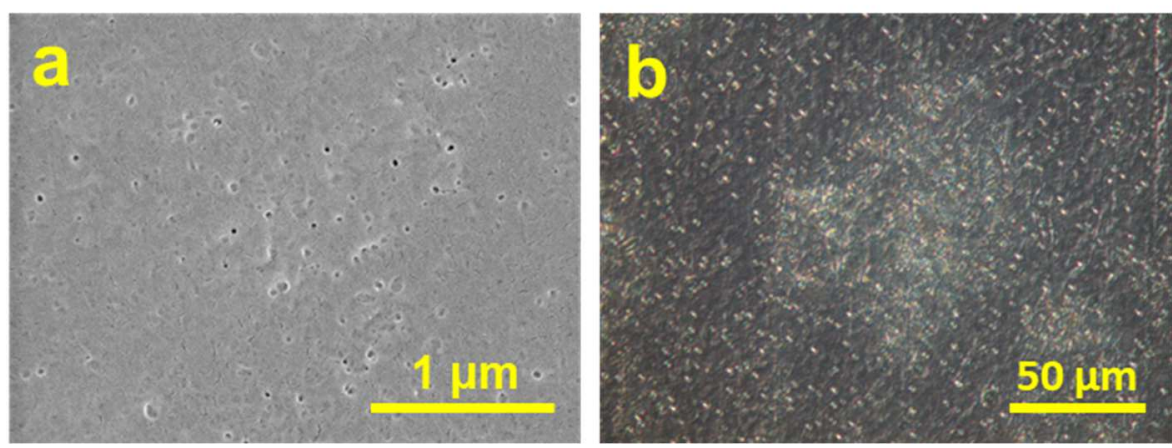
Figure S9. a) SEM image of the top surface of the honeycomb film fabricated with CNWsSU on the surface of Teflon with the concentration of $16 \mathrm{mg} / \mathrm{mL}(7 \mathrm{~mL})$. b) LSM image of the top surface of the honeycomb film fabricated with CNWs-SU on the surface of Teflon with the concentration of $3 \mathrm{mg} / \mathrm{mL}(5 \mathrm{~mL})$.
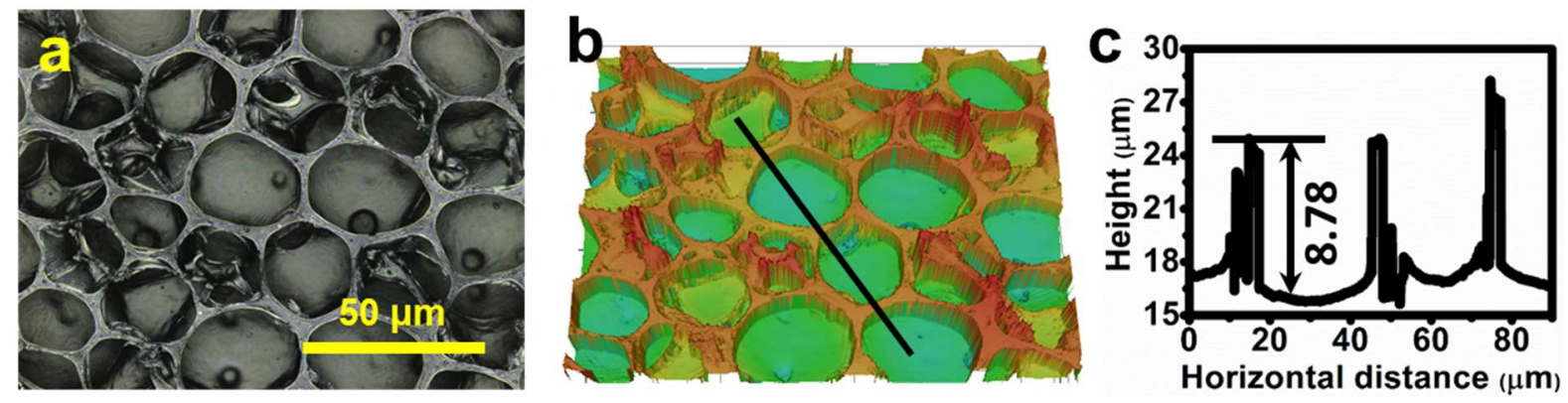

Figure S10. (a) LSM image, (b) 3D LSM image, and (c) height profile of the honeycomb film fabricated with CNWs-SU. Solid substrate: silicon wafer; volume of CNWs-SU solution: 10 mL; solution concentration: $3 \mathrm{mg} / \mathrm{mL}$; relative humidity: $96 \%$.
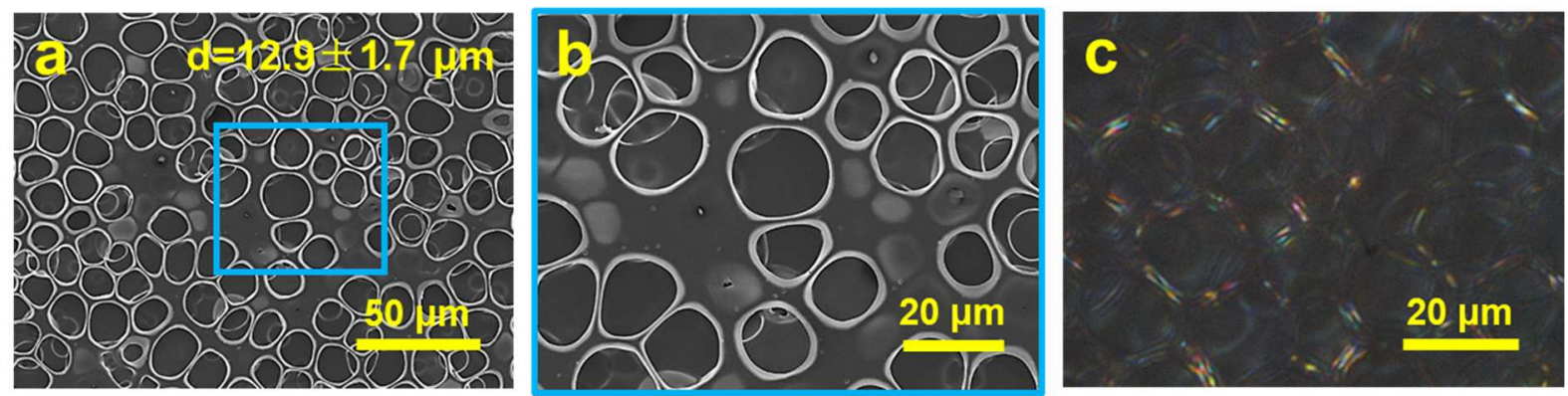

Figure S11. a) Representative SEM image of the top surface of the honeycomb film fabricated with CNWs-SU at $60 \%$ relative humidity. b) SEM image of the magnified region as in (a). c) PLM image of the top surface of the honeycomb film fabricated with CNWs-SU at $60 \%$ relative humidity. 

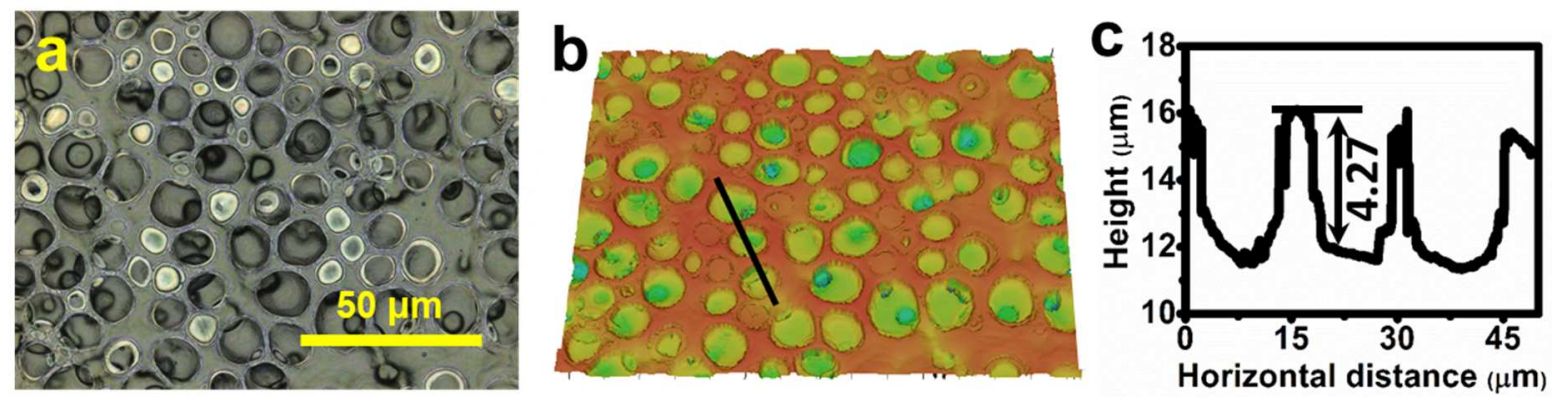

Figure S12. (a) LSM image, (b) 3D LSM image, and (c) height profile of the honeycomb film fabricated with CNWs-SU. Solid substrate: silicon wafer; volume of CNWs-SU solution: 10 $\mathrm{mL}$; solution concentration: $3 \mathrm{mg} / \mathrm{mL}$; relative humidity: $60 \%$.
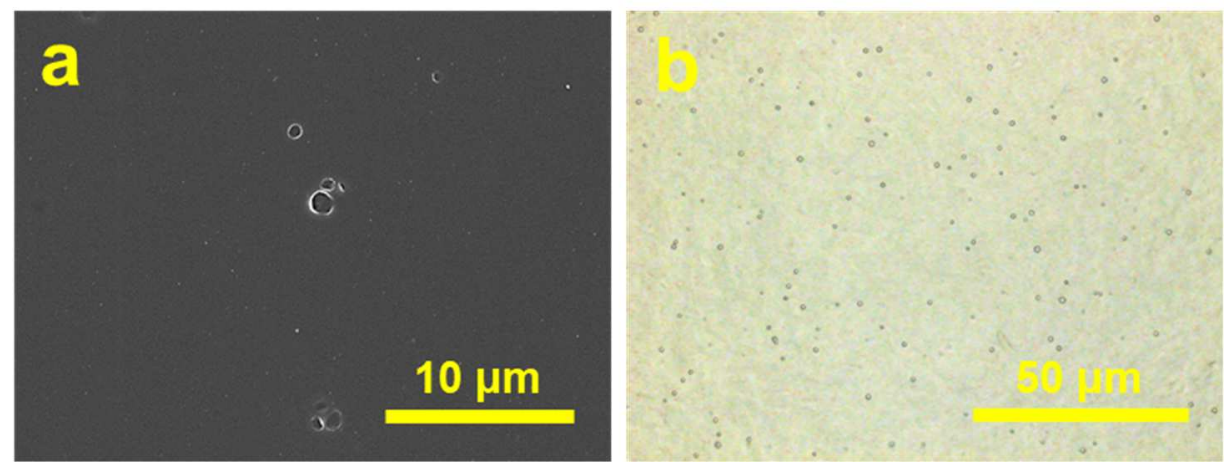

Figure S13. (a) SEM image, and (b) LSM image of the honeycomb film fabricated with CNWs-SU. Solid substrate: silicon wafer; volume of CNWs-SU solution: $10 \mathrm{~mL}$; solution concentration: $3 \mathrm{mg} / \mathrm{mL}$; relative humidity: $45 \%$. 